SUPPORTING INFORMATION

\title{
Total Syntheses of Conjugation-Ready Trisaccharide Repeating Units of Pseudomonas aeruginosa 011 and Staphylococcus aureus Type 5 Capsular Polysaccharide for Vaccine Development
}

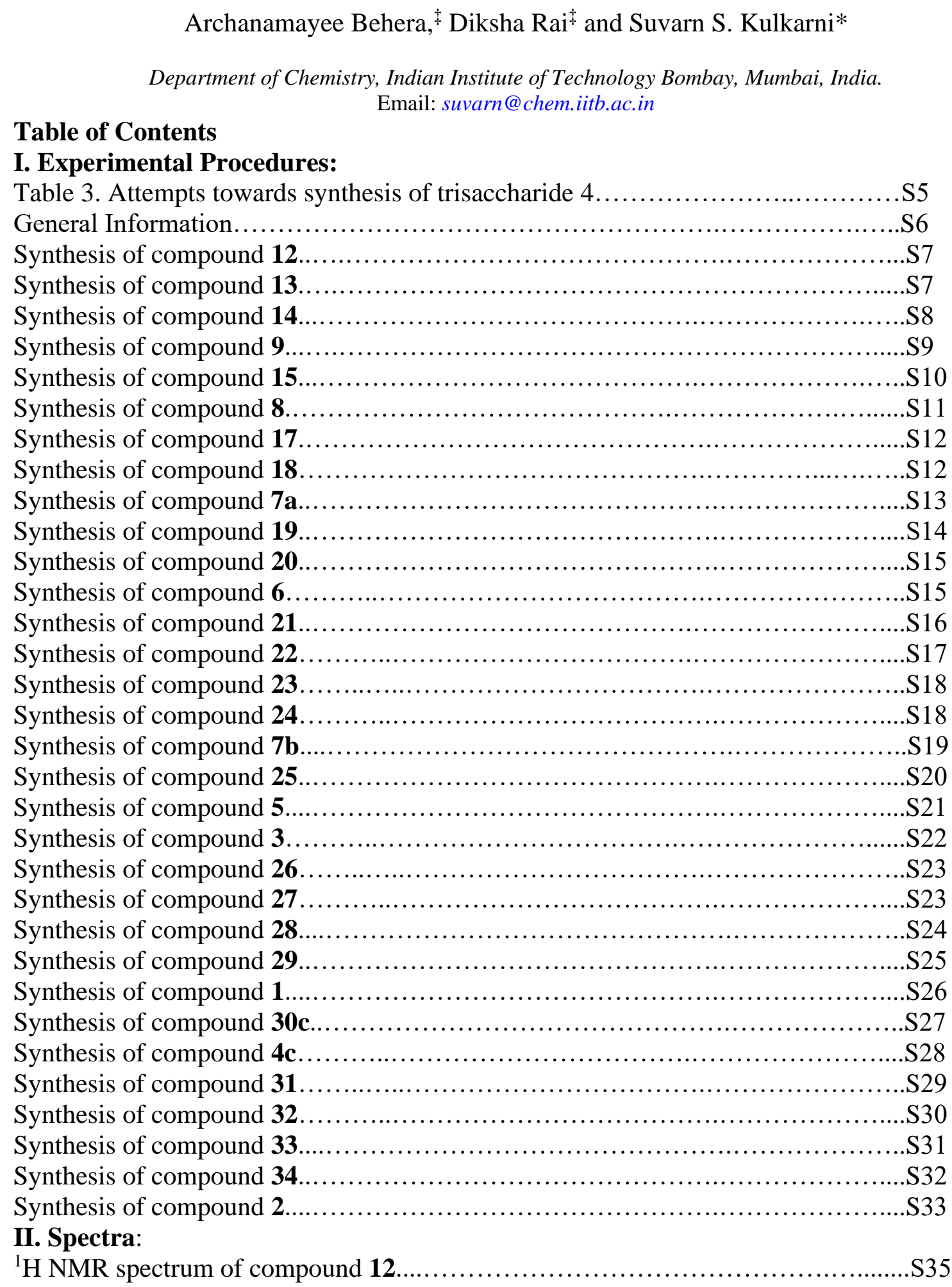




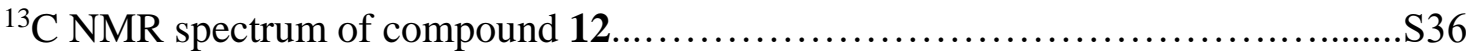

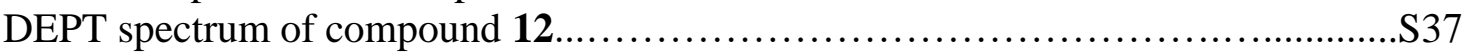

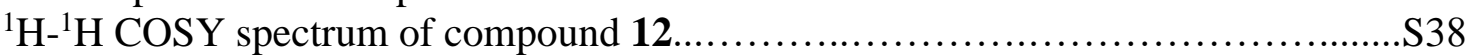

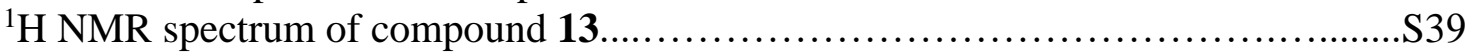

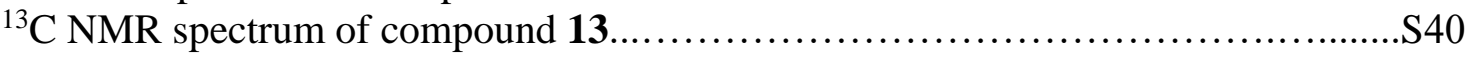

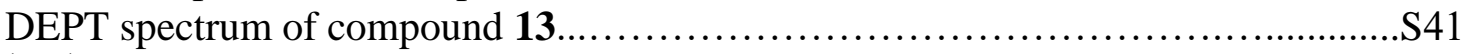

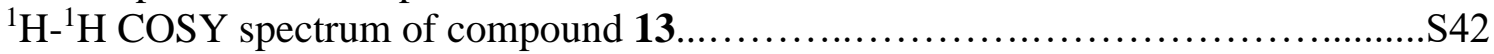

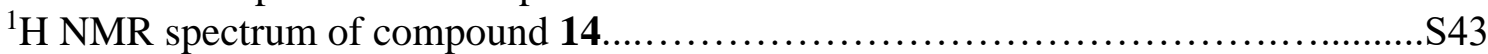

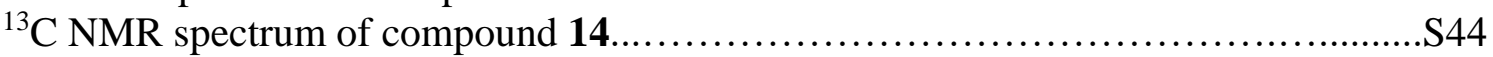

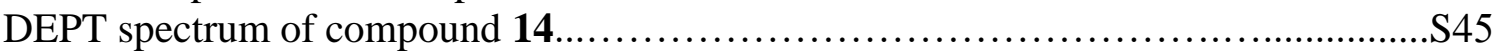

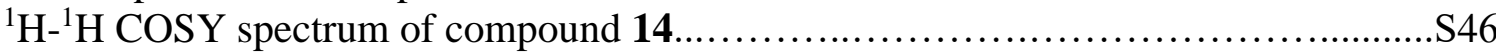

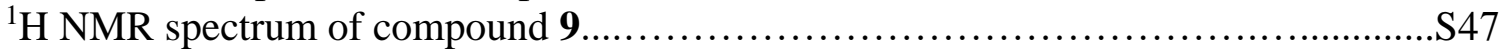

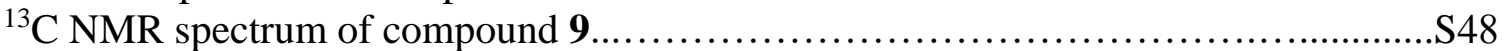

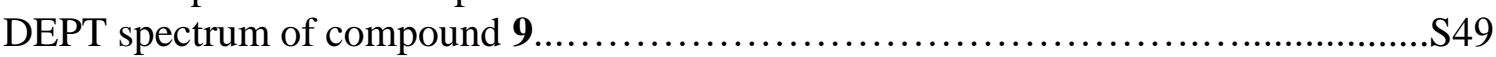

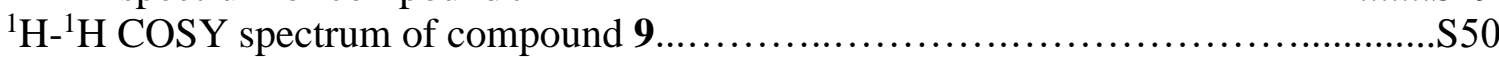

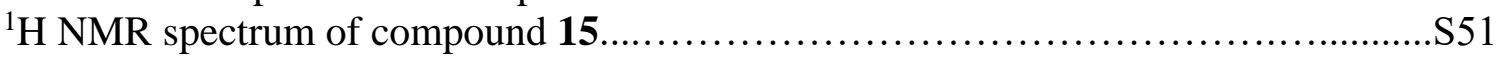

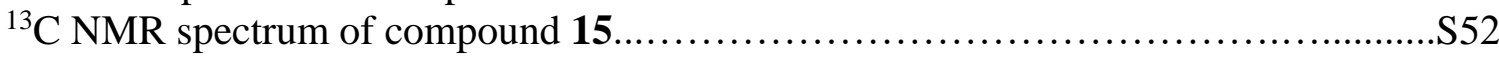

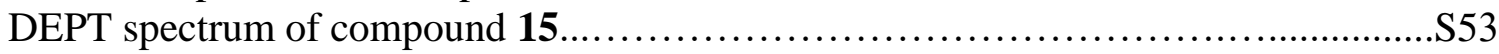

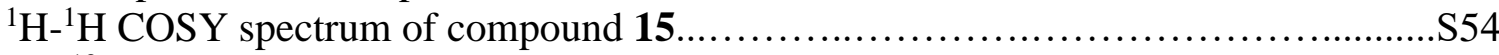

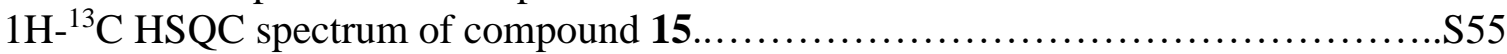

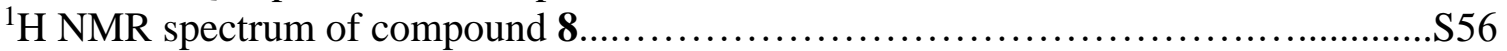

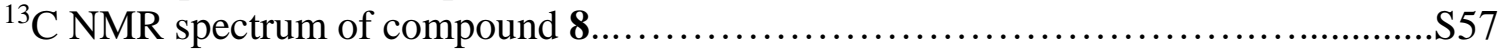

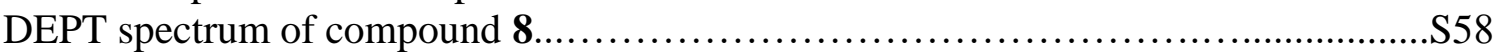

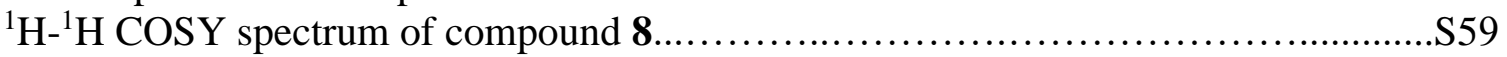

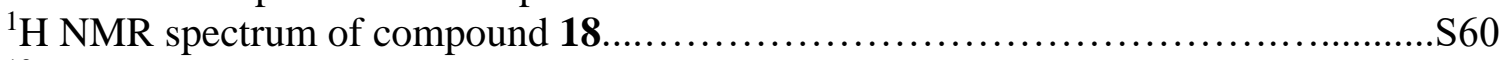

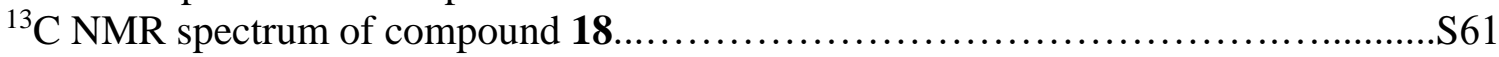

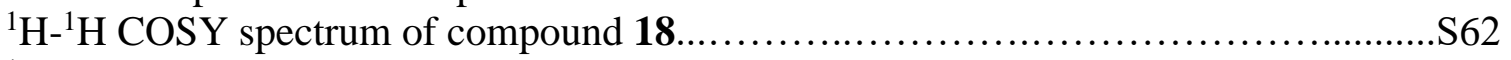

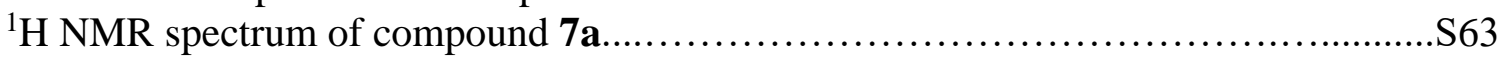

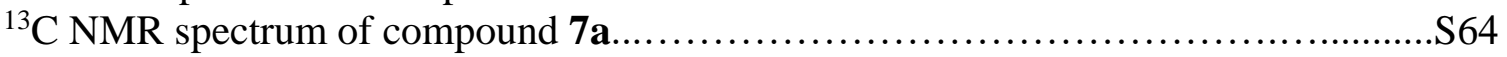

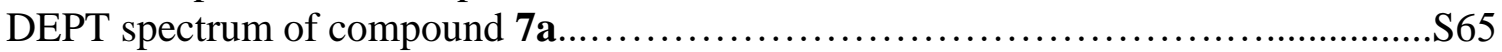

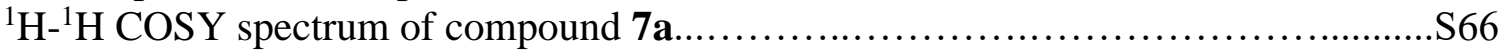

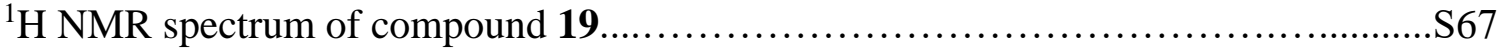

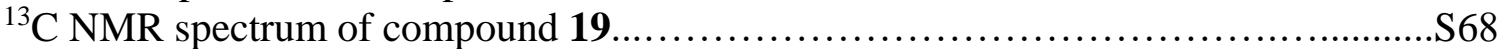

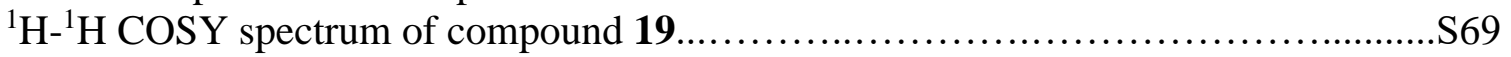

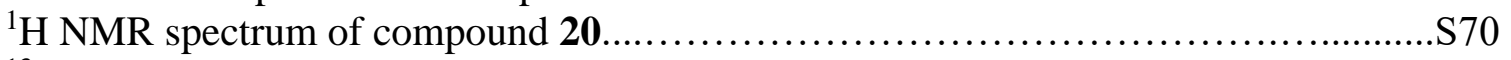

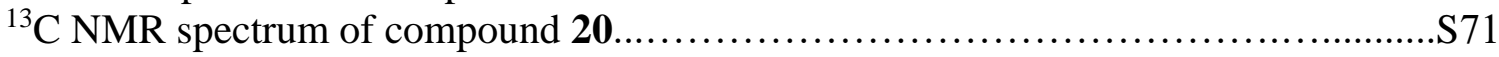

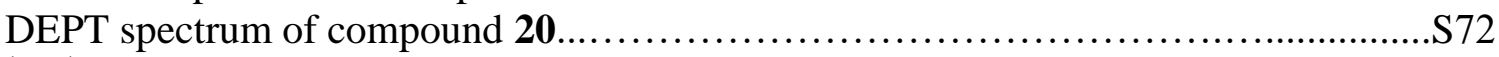

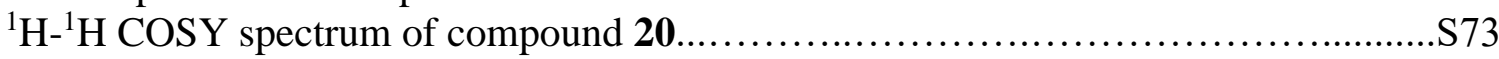

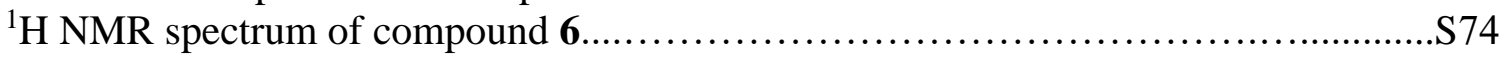

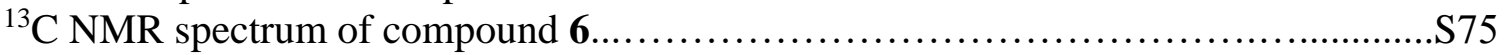

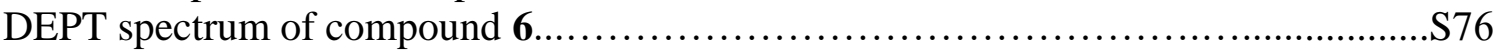

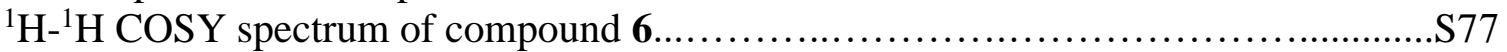

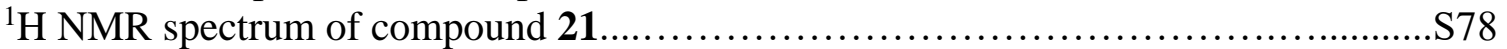

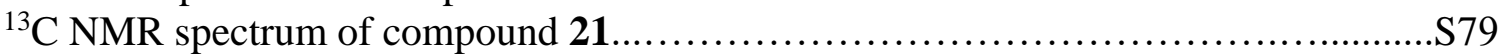

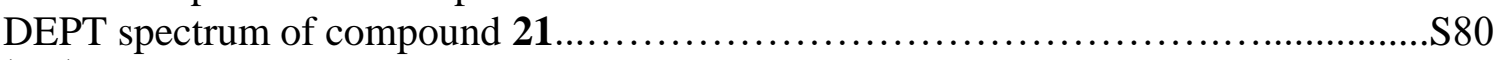

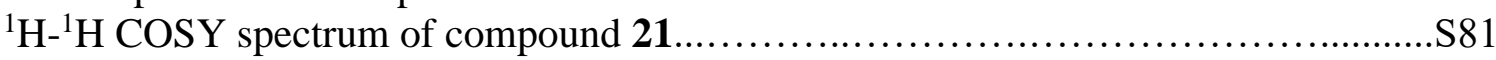

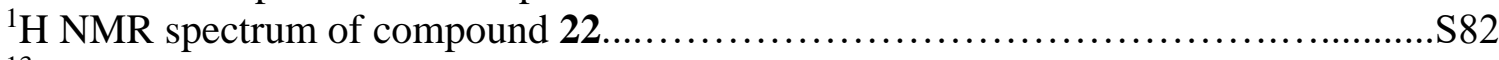

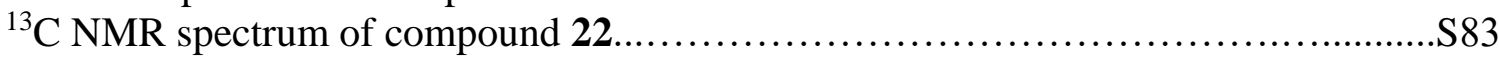

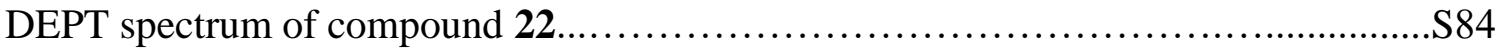

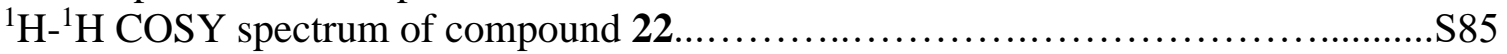




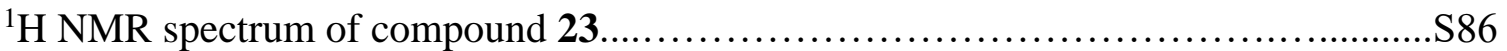

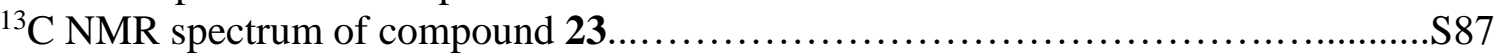

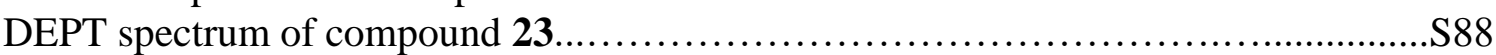

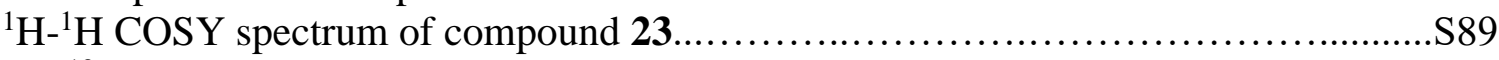

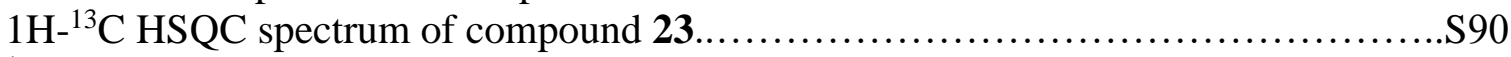

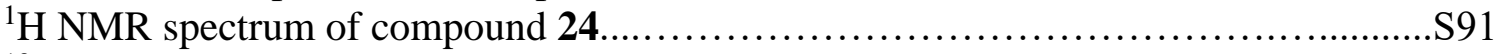

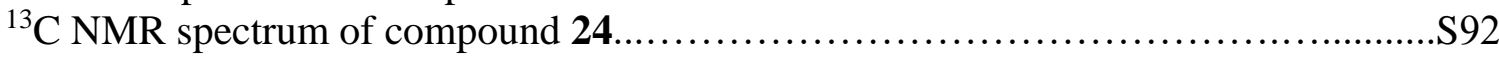

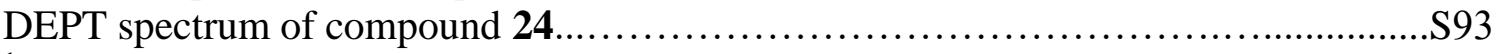

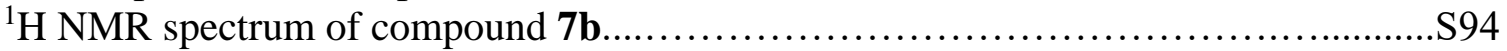

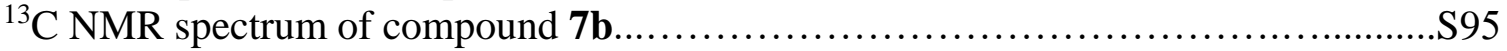

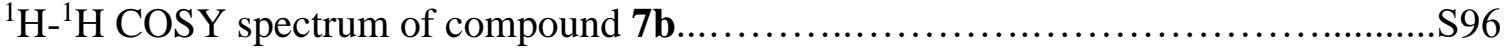

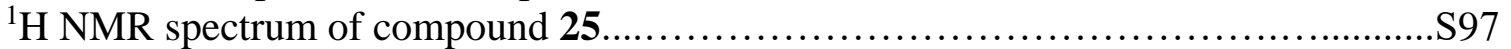

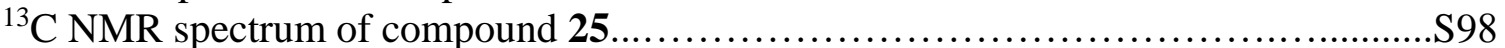

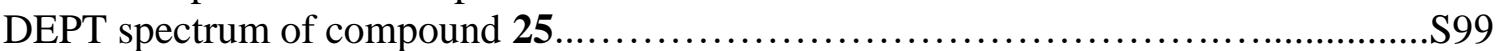

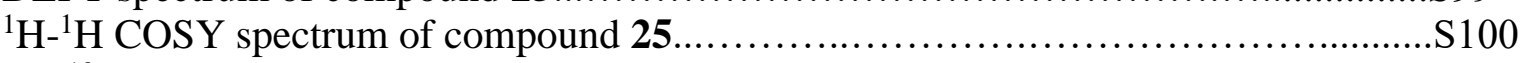

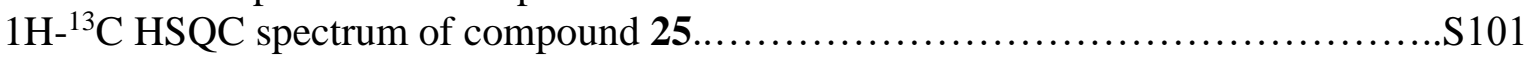

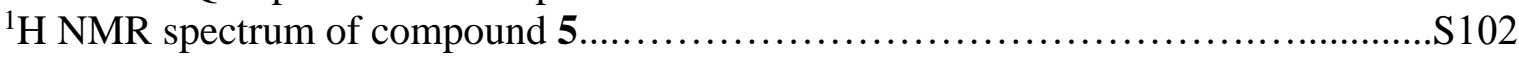

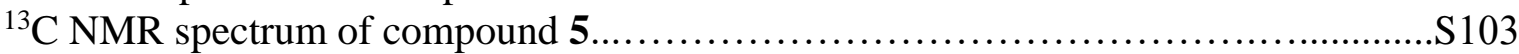

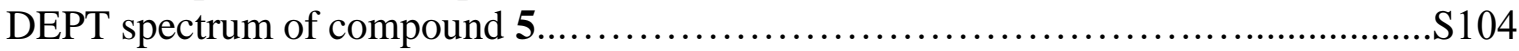

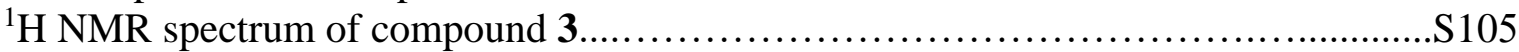

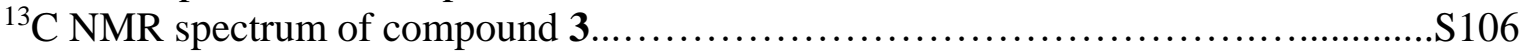

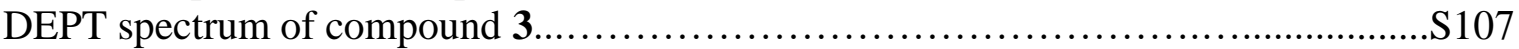

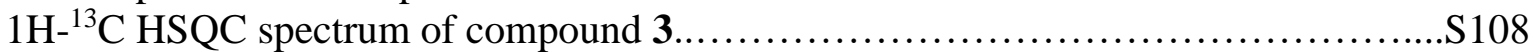

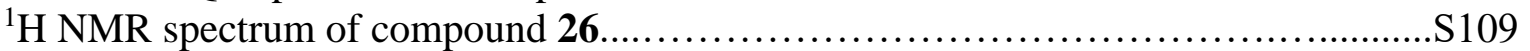

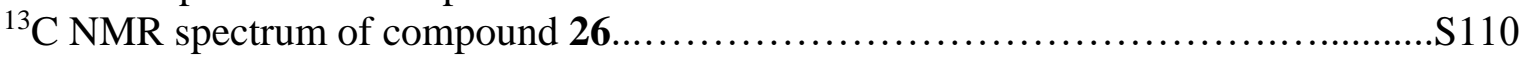

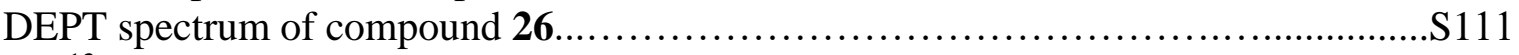

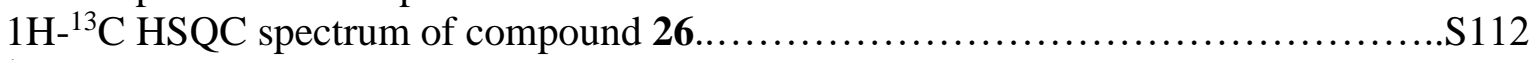

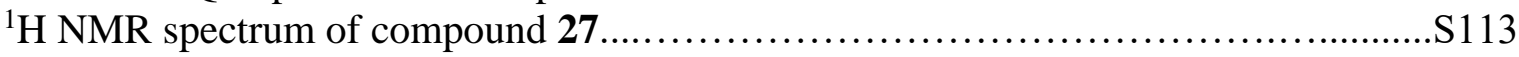

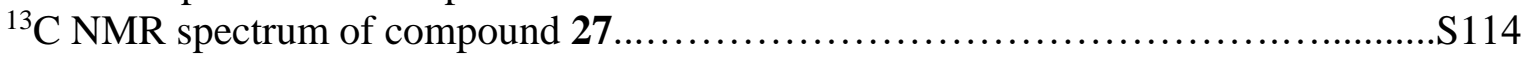

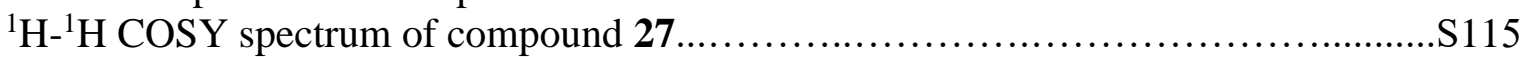

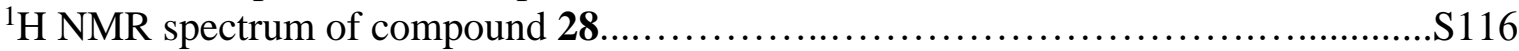

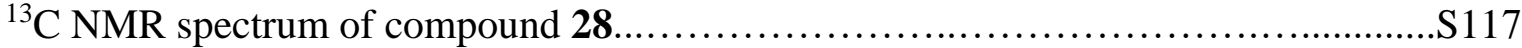

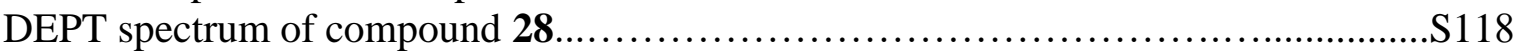

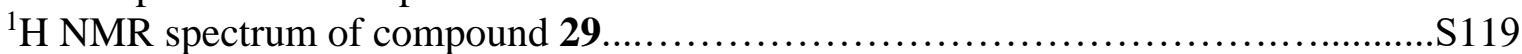

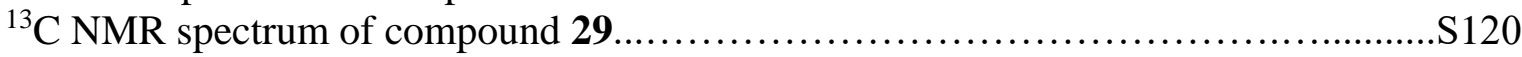

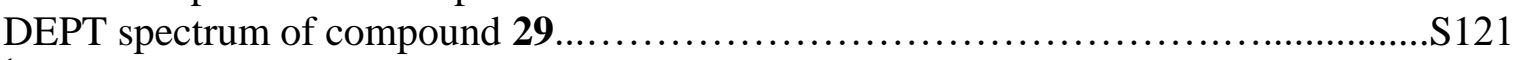

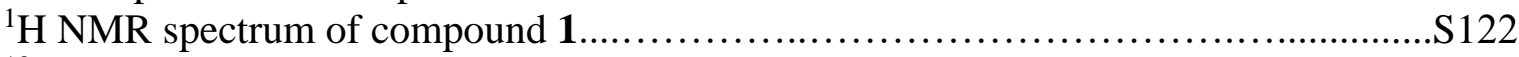

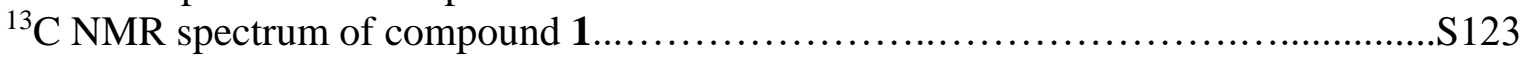

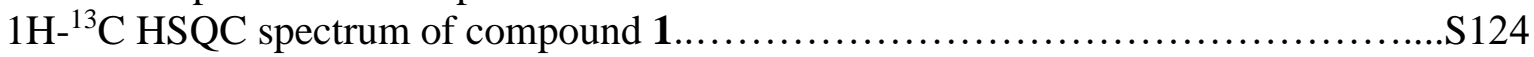

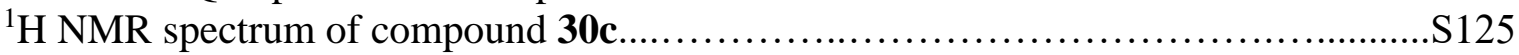

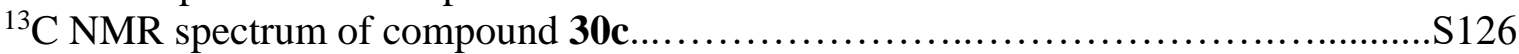

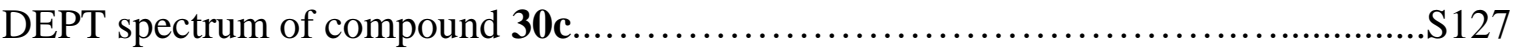

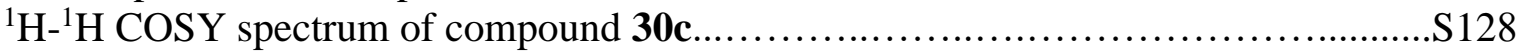

$1 \mathrm{H}^{-13} \mathrm{C}$ HSQC spectrum of compound 30c....................................... S129

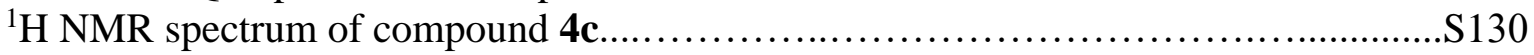

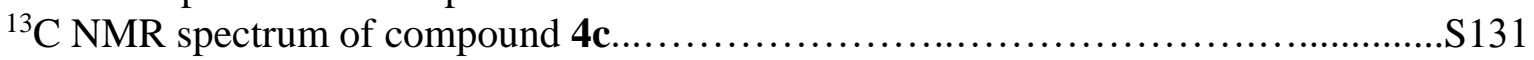

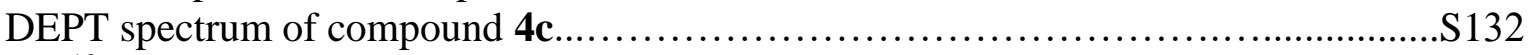

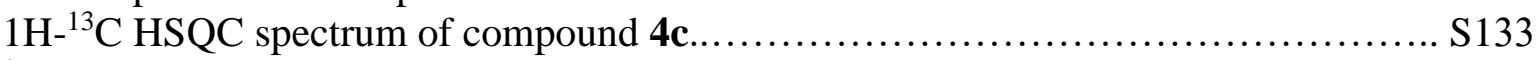

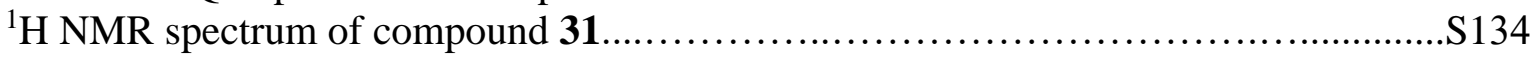

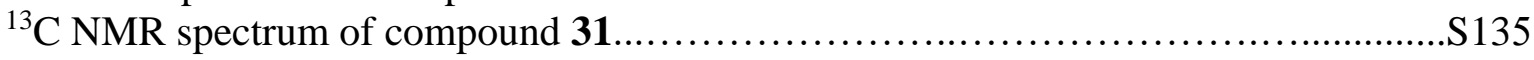




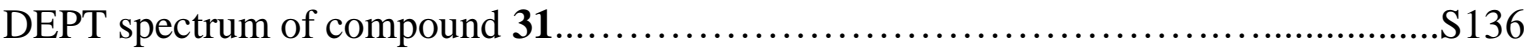

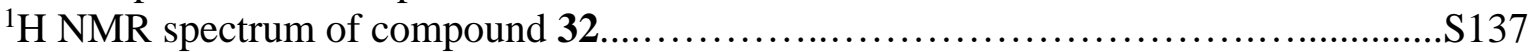

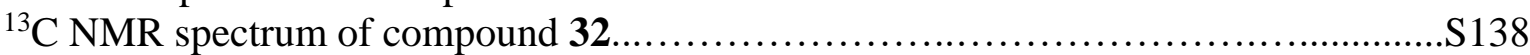

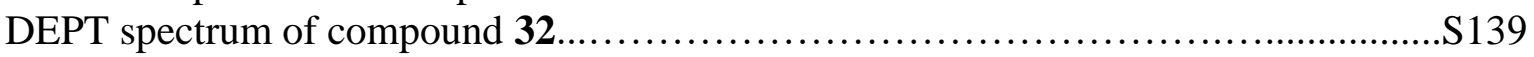

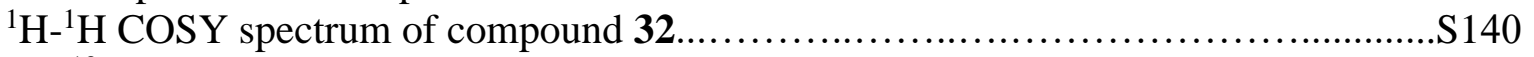

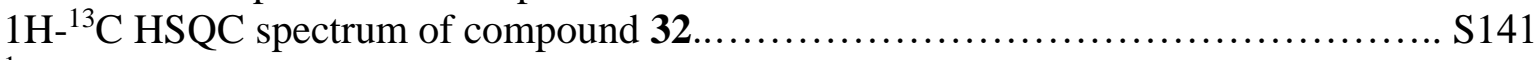

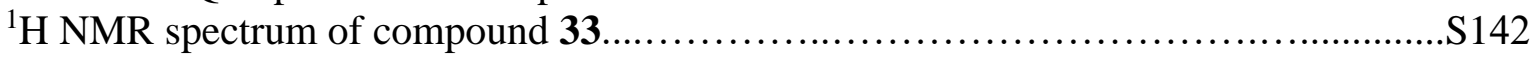

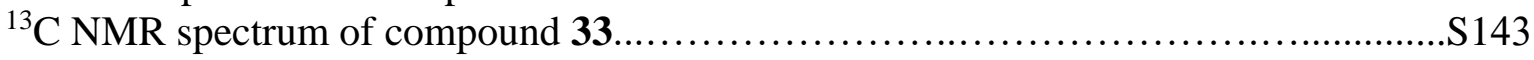

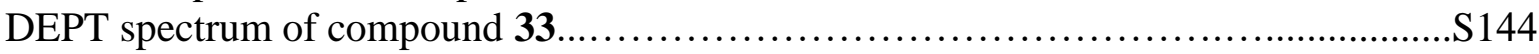

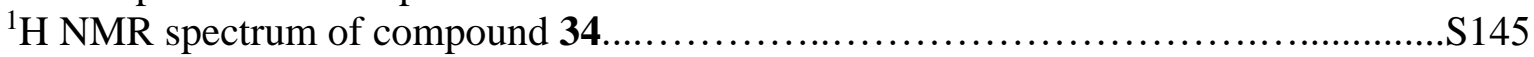

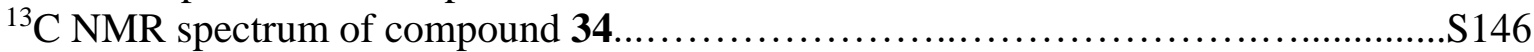

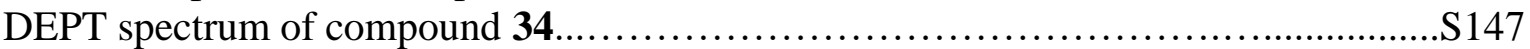

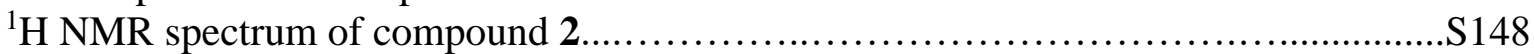

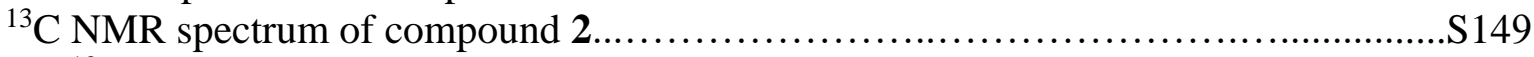

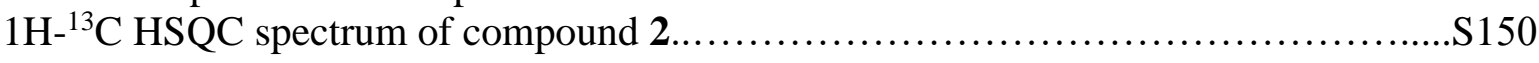


Table 3. Attempts towards synthesis of trisaccharide 4.

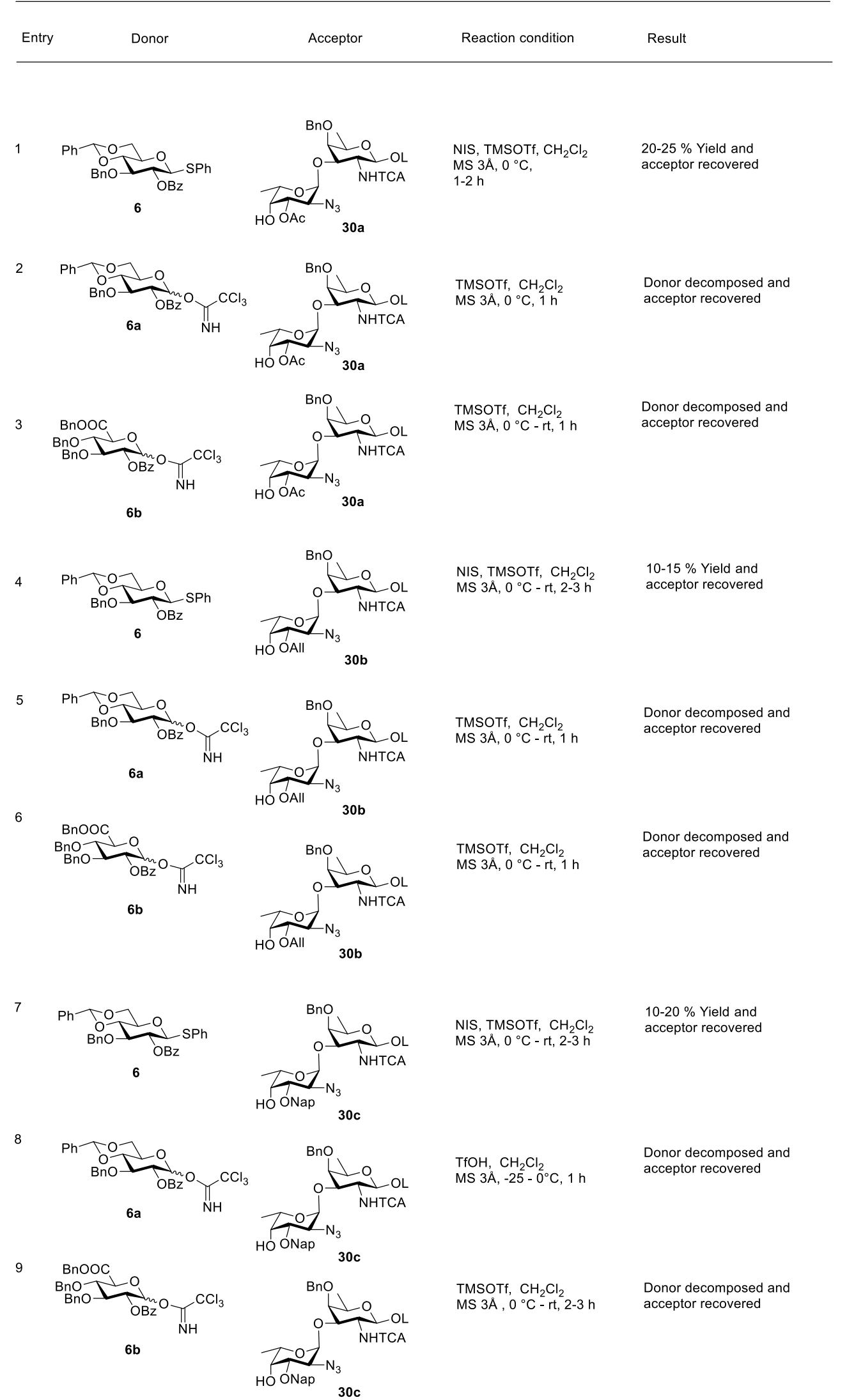




\section{Experimental procedure:}

General informations. All reactions were conducted under the dry nitrogen atmosphere. Solvents $\left(\mathrm{CH}_{2} \mathrm{Cl}_{2}>99 \%\right.$, THF 99.5\%, acetonitrile 99.8\%, DMF 99.5\%) were purchased in capped bottles and dried under sodium or $\mathrm{CaH}_{2}$. All other solvents and reagents were used without further purification. All glassware used was oven dried before use. TLC was performed on pre-coated Aluminium plates of Silica Gel 60 F254 (0.25 mm, E. Merck). Developed TLC plates were visualized under a short-wave UV lamp and by heating plates that were dipped in ammonium molybdate/cerium (IV) sulfate solution. Silica gel column chromatography was performed using Silica Gel (100-200 mesh) and employed a solvent polarity correlated with TLC mobility. We have used 3 Angstrom powdered molecular sieves in our study. The powdered MS were weighed in a dried pear shaped flask and activated by periodic heating of flask by using flame over 15 min period.

NMR experiments were conducted on 500 and $400 \mathrm{MHz}$ instrument using $\mathrm{CDCl}_{3}$ (D, $99.8 \%)$ or $\mathrm{D}_{2} \mathrm{O}(\mathrm{D}, 99.9 \%)$ as solvents. Chemical shifts are relative to the deuterated solvent peaks and are in parts per million (ppm). ${ }^{1} \mathrm{H}-{ }^{1} \mathrm{H}$ COSY was used to confirm proton assignments. Mass spectra were acquired in the ESI mode. Melting points were determined by capillary apparatus. Specific rotation experiments were measured at $589 \mathrm{~nm}(\mathrm{Na})$ and 25 ${ }^{\circ} \mathrm{C}$. IR spectra were recorded on an FT-IR spectrometer. 


\section{Phenyl-3-O-naphthylmethyl-6-deoxy-1-thio- $\beta$-D-mannopyranoside (12)}

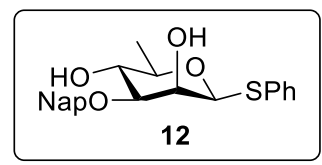

Triol 9 (1.0 g, $3.9 \mathrm{mmol})$ was dissolved in dry toluene $(26 \mathrm{~mL})$, and to this stirred solution, $\mathrm{Bu}_{2} \mathrm{SnO}(1.14 \mathrm{~g}, 4.6 \mathrm{mmol})$ was added and reaction mixture was kept at $110^{\circ} \mathrm{C}$ for $8 \mathrm{~h}$. After completion of the reaction, solvent was removed under reduced pressure, the crude compound was kept under vacuum for $5 \mathrm{~h}$.

Above crude compound was dissolved in dry toluene $(15 \mathrm{~mL})$, and heated at $60{ }^{\circ} \mathrm{C}$. At this temperature TBAB (1.9 g, $5.85 \mathrm{mmol})$ followed by $\mathrm{NapBr}(1.0 \mathrm{~g}, 4.68 \mathrm{mmol})$ were added and the reaction mixture was kept for stirring at same temperature for $6 \mathrm{~h}$. After completion of the reaction (confirmed by TLC), the reaction was diluted with EtOAc $(2 \times 50$ $\mathrm{mL}$ ) and organic layer was washed with brine solution twice, dried over anhydrous $\mathrm{Na}_{2} \mathrm{SO}_{4}$, organic layer was concentrated, and purified by column chromatography on silica gel (2:3 ethyl acetate: pet ether) to obtain $\mathbf{1 2}$ as a white solid (1.27 g, 82\%).

$[\alpha]^{25} \mathrm{D}-34.24\left(c=0.5, \mathrm{CHCl}_{3}\right)$. M.P. $135^{\circ} \mathrm{C}$.

IR $\left(\mathrm{cm}^{-1}, \mathrm{CHCl}_{3}\right) v 3428,2932,2342,1064,751$.

${ }^{1} \mathrm{H}$ NMR (500 MHz, $\left.\mathrm{CDCl}_{3}\right) \delta$ 7.88-7.80 (m, 4H, ArH), 7.53-7.50 (m, 5H, ArH), 7.33-7.28 (m, 3H, ArH), 4.93 (d, J = 12.0 Hz, 1H, $\left.\mathrm{CH}_{2} \mathrm{Ph}\right), 4.80$ (s, 1H, H-1), 4.74 (d, J = $12.0 \mathrm{~Hz}, 1 \mathrm{H}$, $\mathrm{CH}_{2} \mathrm{Ph}$ ), 4.33 (bs, 1H, H-2), 3.69 (d, $\left.J=10.0 \mathrm{~Hz}, 1 \mathrm{H}, \mathrm{H}-4\right), 3.43$ (dd, $J=9.2 \mathrm{~Hz}, 3.2 \mathrm{~Hz}, 1 \mathrm{H}$, H-3), 3.36-3.33 (m, 1H, H-5), 2.70 (bs, 1H, OH), 2.43 (bs, 1H, OH), 1.41 (d, $J=6.0 \mathrm{~Hz}, 3 \mathrm{H}$, $\left.\mathrm{CH}_{3}\right)$.

${ }^{13} \mathrm{C} \mathrm{NMR}\left(125 \mathrm{MHz}, \mathrm{CDCl}_{3}\right) \delta 134.9,134.8,133.4,133.3,131.2,129.1,128.9,128.1,127.9$, $127.5,127.2,126.6,126.5,125.8,86.8,82.1,76.4,71.7,71.5,69.7,18.2$.

HR-ESI-MS (m/z): calcd for $\mathrm{C}_{23} \mathrm{H}_{24} \mathrm{O}_{4} \mathrm{SNa}^{+}\left(\mathrm{M}+\mathrm{Na}^{+}\right)$: 419.1288, found: 419.1281 .

Phenyl-2-azido-3- $O$-naphthylmethyl-2,6-dideoxy-1-thio- $\beta$-D-galactopyranoside (13)

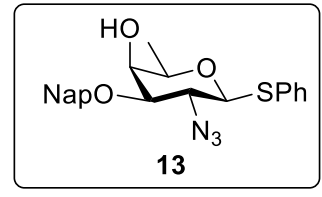


$\mathrm{Tf}_{2} \mathrm{O}(3.3 \mathrm{~mL}, 19.5 \mathrm{mmol})$ and dry pyridine $(3.3 \mathrm{~mL}, 41.5 \mathrm{mmol})$ were added sequentially at $0{ }^{\circ} \mathrm{C}$ to a stirred solution of $\mathbf{1 2}(1.28 \mathrm{~g}, 3.22 \mathrm{mmol})$ in $\mathrm{CH}_{2} \mathrm{Cl}_{2}(67 \mathrm{~mL})$. Then the reaction mixture was stirred at $0{ }^{\circ} \mathrm{C}$ for 15 minutes. After complete consumption of the starting material, the reaction mixture was diluted with $\mathrm{CH}_{2} \mathrm{Cl}_{2}$ and washed successively with $1 \mathrm{~N}$ $\mathrm{HCl}$, aq. $\mathrm{NaHCO}_{3}$ and brine. Separated organic layer was dried over anhydrous $\mathrm{Na}_{2} \mathrm{SO}_{4}$, concentrated and dried under high vacuum.

The crude product which was obtained after removal of solvent, was dissolved in acetonitrile $(67 \mathrm{~mL})$ and to this, $\operatorname{TBAN}_{3}(0.82 \mathrm{~g}, 3.22)$ was added at $-30{ }^{\circ} \mathrm{C}$ and the reaction was stirred at the same temperature for $20 \mathrm{~h} . \mathrm{TBANO}_{2}(2.80 \mathrm{~g}, 9.66 \mathrm{mmol})$ was added and the reaction mixture was stirred at $\mathrm{rt}$ for another $12 \mathrm{~h}$. The reaction mixture was diluted with EtOAc $(2 \times 60 \mathrm{~mL})$ and washed with water. Separated organic layer was dried over anhydrous $\mathrm{Na}_{2} \mathrm{SO}_{4}$ and concentrated in vacuo. The crude product was purified by column chromatography on silica gel (1:9 ethyl acetate: pet ether) to obtain $\mathbf{1 3}$ as yellowish viscous liquid $(0.81 \mathrm{~g}, 60 \%)$.

$[\alpha]^{25} \mathrm{D}-53.38\left(c=0.45, \mathrm{CHCl}_{3}\right)$.

IR $\left(\mathrm{cm}^{-1}, \mathrm{CHCl}_{3}\right) v 3479,2919,2330,2112,1366,1281,1064,821,765,692$.

${ }^{1} \mathrm{H}$ NMR (500 MHz, $\left.\mathrm{CDCl}_{3}\right) \delta$ 7.87-7.80 (m, 5H, ArH), 7.63-7.26 (m, 7H, ArH), 4.87-4.81 $\left(\mathrm{m}, 2 \mathrm{H},-\mathrm{CH}_{2} \mathrm{Ph}\right), 4.35$ (d, $\left.J=10.5 \mathrm{~Hz}, 1 \mathrm{H}, \mathrm{H}-1\right), 3.79$ (d, $\left.J=3.0 \mathrm{~Hz}, 1 \mathrm{H}, \mathrm{H}-4\right), 3.64$ (t, $J=$ $9.3 \mathrm{~Hz}, 1 \mathrm{H}, \mathrm{H}-2), 3.51-3.47$ (m, 1H, H-5), 3.43 (dd, $J=9.5 \mathrm{~Hz}, 3.1 \mathrm{~Hz}, 1 \mathrm{H}, \mathrm{H}-3), 2.33$ (bs, $1 \mathrm{H}, \mathrm{OH}), 1.37\left(\mathrm{~d}, J=6.5 \mathrm{~Hz}, 3 \mathrm{H}, \mathrm{CH}_{3}\right)$.

${ }^{13} \mathrm{C} \mathrm{NMR}\left(100 \mathrm{MHz}, \mathrm{CDCl}_{3}\right) \delta 134.6,133.5,133.4$ 131.8, 129.2, 128.8, 128.4, 128.1, 127.9, $127.3,126.6,126.5,125.9,86.3,81.4,74.6,72.3,68.4,61.6,17.0$.

HR-ESI-MS (m/z): calcd for $\mathrm{C}_{23} \mathrm{H}_{23} \mathrm{~N}_{3} \mathrm{O}_{3} \mathrm{SNa}^{+}\left(\mathrm{M}+\mathrm{Na}^{+}\right)$: 444.1352, found: 444.1359.

\section{Phenyl-2-azido-3- $O$-naphthylmethyl-4- $O$-benzyl-2,6-dideoxy-1-thio- $\beta$-D-galacto pyranoside (14)}

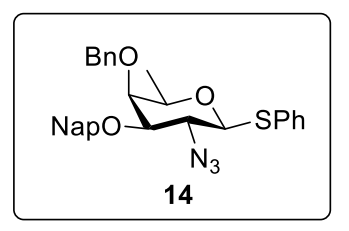

$\mathrm{NaH} 60 \%$ wt $(0.28 \mathrm{~g}, 11.37 \mathrm{mmol})$ was added to a stirred solution of $\mathbf{1 3}$ (1.6 g, $3.8 \mathrm{mmol})$ in DMF $(24 \mathrm{~mL})$ at $0{ }^{\circ} \mathrm{C}$, after $5 \mathrm{~min}$ benzyl bromide $(0.7 \mathrm{~mL}, 5.7 \mathrm{mmol})$ was added drop wise 
and the reaction mixture was allowed to stir for $1 \mathrm{~h}$. After consumption of the starting material, the reaction mixture was diluted with EtOAc $(2 \times 50 \mathrm{~mL})$ and washed with brine. Separated organic layer was dried over anhydrous $\mathrm{Na}_{2} \mathrm{SO}_{4}$ and concentrated in vacuo. The crude product was purified by column chromatography on silica gel (5\% ethyl acetate: pet ether) to obtain 14 as pale yellow foam $(1.80 \mathrm{~g}, 94 \%)$.

$[\alpha]^{25} \mathrm{D}-13.23\left(c=0.6, \mathrm{CHCl}_{3}\right)$.

IR $\left(\mathrm{cm}^{-1}, \mathrm{CHCl}_{3}\right) v 2112,1355,1281,1065,791,751,695$.

${ }^{1} \mathrm{H}$ NMR (400 MHz, $\left.\mathrm{CDCl}_{3}\right) \delta$ 7.88-7.83 (m, 4H, ArH), 7.64-7.24 (m, 13H, ArH), 4.98 (d, $J=$ $\left.11.6 \mathrm{~Hz}, 1 \mathrm{H}, \mathrm{CH}_{2} \mathrm{Ph}\right), 4.89$ (bs, 2H, $\left.\mathrm{CH}_{2} \mathrm{Ph}\right), 4.65$ (d, $\left.J=11.2 \mathrm{~Hz}, 1 \mathrm{H}, \mathrm{CH}_{2} \mathrm{Ph}\right), 4.39$ (d, $J=$ $10.0 \mathrm{~Hz}, 1 \mathrm{H}, \mathrm{H}-1), 3.89$ (t, $J=9.8 \mathrm{~Hz}, 1 \mathrm{H}, \mathrm{H}-2), 3.63-3.62$ (m, 1H, H-4), 3.50-3.46 (m, 2H, $\mathrm{H}-3, \mathrm{H}-5), 1.30$ (d, $\left.J=6.4 \mathrm{~Hz}, 3 \mathrm{H},-\mathrm{CH}_{3}\right)$.

${ }^{13} \mathrm{C} \mathrm{NMR}\left(100 \mathrm{MHz}, \mathrm{CDCl}_{3}\right) \delta 138.5,135.1,133.3 ., 133.2,132.8,132.2,128.9,128.4,128.3$, $128.0,127.9,127.8,127.6,126.7,126.4,126.2$, 125.8, 86.3, 82.7, 75.3, 74.8, 74.7, 72.6, 61.5, 17.4 .

HR-ESI-MS (m/z): calcd for $\mathrm{C}_{30} \mathrm{H}_{29} \mathrm{~N}_{3} \mathrm{O}_{3} \mathrm{SNa}^{+}\left(\mathrm{M}+\mathrm{Na}^{+}\right)$: 534.1822, found: 534.1818.

Phenyl-(trichloroacetyl carbonylamino)-4- $O$-benzyl-2,6-dideoxy-3- $O$-naphthylmethyl-1thio- $\beta$-D-galactopyranoside (9)

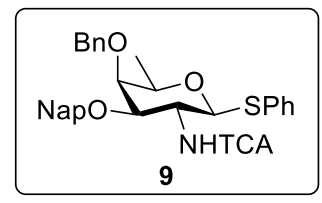

Zn dust (2.2 g) and $1.5 \mathrm{~mL}$ of AcOH was added to a stirred solution of 14 (1.5 g, $2.93 \mathrm{mmol})$ in $10.6 \mathrm{~mL}$ of THF, and the solution was stirred at $\mathrm{rt}$ for $4 \mathrm{~h}$. After complete conversion of the starting material, the reaction mixture was filtered through Celite bed and concentrated under vacuo.

The residue obtained after solvent removal was dissolved in THF $(20.7 \mathrm{~mL})$ and $3 \AA$ MS (3.0 g) were added and TCACl $(0.7 \mathrm{~mL}, 5.86 \mathrm{mmol})$ was added slowly at $0{ }^{\circ} \mathrm{C}$. After stirring the reaction mixture at same temperature for $1 \mathrm{~h}$, it was diluted with $\mathrm{CH}_{2} \mathrm{Cl}_{2}(2 \times 20$ $\mathrm{mL}$ ) and washed with brine. Separated organic layer was dried over anhydrous $\mathrm{Na}_{2} \mathrm{SO}_{4}$, concentrated and purified by column chromatography on silica gel (15\% ethyl acetate: pet ether) to afford 9 as a white solid (1.62 $\mathrm{g}, 88 \%)$. 
$[\alpha]^{25} \mathrm{D}+30.70\left(c=0.65, \mathrm{CHCl}_{3}\right)$. M.P. $168^{\circ} \mathrm{C}$.

IR $\left(\mathrm{cm}^{-1}, \mathrm{CHCl}_{3}\right) v 3747,3324,2877,2342,1688,1532,1068,820,755$.

${ }^{1} \mathrm{H}$ NMR (400 MHz, $\left.\mathrm{CDCl}_{3}\right) \delta$ 7.83-7.74 (m, 4H, ArH), 7.57-6.87 (m, 14H, ArH), $5.26(\mathrm{~d}, J=$ $10.0 \mathrm{~Hz}, 1 \mathrm{H}, \mathrm{H}-1), 4.96$ (d, $\left.J=11.2 \mathrm{~Hz}, 1 \mathrm{H}, \mathrm{CH}_{2} \mathrm{Ph}\right), 4.80$ (d, $\left.J=11.6 \mathrm{~Hz}, 1 \mathrm{H}, \mathrm{CH}_{2} \mathrm{Ph}\right), 4.73$ $\left(\mathrm{d}, J=11.2 \mathrm{~Hz}, 1 \mathrm{H}, \mathrm{CH}_{2} \mathrm{Ph}\right), 4.63\left(\mathrm{~d}, J=11.6 \mathrm{~Hz}, 1 \mathrm{H}, \mathrm{CH}_{2} \mathrm{Ph}\right), 4.35(\mathrm{dd}, J=10.4 \mathrm{~Hz}, 2.4 \mathrm{~Hz}$, 1H, H-3), 3.97-3.90 (m, 1H, H-2), 3.72-3.63 (m, 2H, H-4, H-5), 1.31 (d, J = 6.4 Hz, 3H, $\left.\mathrm{CH}_{3}\right)$.

${ }^{13} \mathrm{C} \mathrm{NMR}\left(100 \mathrm{MHz}, \mathrm{CDCl}_{3}\right) \delta 161.9,138.6,135.0,133.4,133.3,133.0,132.6,129.2,128.7$, $128.5,128.1,128.0,127.9,127.8,127.2,126.5,126.4,126.1,92.8,84.2,78.8,75.9,75.1$, $74.9,72.8,53.9,17.6$.

HR-ESI-MS (m/z): calcd for $\mathrm{C}_{32} \mathrm{H}_{30} \mathrm{NCl}_{3} \mathrm{O}_{4} \mathrm{SK}^{+}(\mathrm{M}+\mathrm{K})^{+}$: 670.0569, found: 670.0593 .

$\mathrm{N}$-benzyloxycarbonyl-3-aminopropanyl phenyl-(trichloroacetyl carbonylamino)-4-O benzyl-2,6-dideoxy-3- $O$-naphthylmethyl-1- $\beta$-D-galactopyranoside (15)

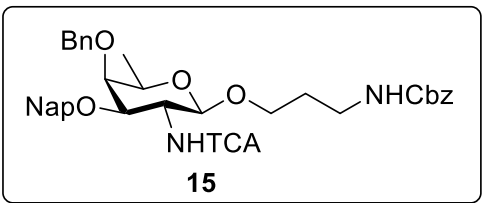

TMSOTf ( $86 \mu \mathrm{L}, 0.48 \mathrm{mmol})$ was added drop wise to a solution of 9 (1.52 g, $2.40 \mathrm{mmol})$, linker 10 (0.60 g, $2.90 \mathrm{mmol}), 3 \AA \mathrm{MS}(1.6 \mathrm{~g})$ and NIS (1.07 g, $4.8 \mathrm{mmol})$ in $\mathrm{CH}_{2} \mathrm{Cl}_{2}(30 \mathrm{~mL})$, at $0{ }^{\circ} \mathrm{C}$. After $30 \mathrm{~min}$ the reaction mixture was diluted with $\mathrm{CH}_{2} \mathrm{Cl}_{2}$, filtered through Celite, and the organic layer was washed with aq. $\mathrm{Na}_{2} \mathrm{~S}_{2} \mathrm{O}_{3}$ solution, dried over anhydrous $\mathrm{Na}_{2} \mathrm{SO}_{4}$ and concentrated. The residue was purified by column chromatography on silica gel $(2: 8$ ethyl acetate: pet ether) to give the desired product $\mathbf{1 5}$ as a white solid $(89 \%, 1.59 \mathrm{~g})$.

$[\alpha]^{25} \mathrm{D}+6.25\left(c=0.5, \mathrm{CHCl}_{3}\right)$. M.P. $186^{\circ} \mathrm{C}$.

IR $\left(\mathrm{cm}^{-1}, \mathrm{CHCl}_{3}\right) v 3322,2934,2346,1693,1537,1262,1072,822,753$.

${ }^{1} \mathrm{H}$ NMR $\left(500 \mathrm{MHz}, \mathrm{CDCl}_{3}\right) \delta$ 7.87-7.81 (m, 4H, ArH), 7.53-7.20 (m, 13H, ArH), 5.19 (bs, 1H, NH), 5.12-5.08 (m, 2H, $\mathrm{CH}_{2}$ of Cbz), 4.99 (d, $\left.J=12.0 \mathrm{~Hz}, 1 \mathrm{H}, \mathrm{CH}_{2} \mathrm{Ph}\right), 4.85-4.81(\mathrm{~m}$, $\left.2 \mathrm{H}, \mathrm{H}-1, \mathrm{CH}_{2} \mathrm{Ph}\right), 4.76-4.72$ (m, 2H, $\left.\mathrm{CH}_{2} \mathrm{Ph}\right), 4.18-4.15$ ( m, 1H, H-3), 4.01-3.94 (m, 2H, H- 
2, $\mathrm{CH}), 3.70$ (bs, 1H, H-4), 3.55-3.52 (m, 2H, CH), 3.38-3.36 (m, 1H, CH), 3.26-3.23 (m, 1H, $\mathrm{CH}), 1.79-1.74(\mathrm{~m}, 2 \mathrm{H}), 1.25\left(\mathrm{~d}, J=6.5 \mathrm{~Hz}, 3 \mathrm{H}, \mathrm{CH}_{3}\right)$.

${ }^{13} \mathrm{C}$ NMR $\left(125 \mathrm{MHz}, \mathrm{CDCl}_{3}\right) \delta 162.3,156.6,138.4,135.1,133.4,133.2,128.63,128.60$, $128.5,128.4,128.1,128.0,127.9,127.0,126.4,126.3,126.0,99.6,92.8,78.3,75.1,74.9$, $72.7,70.9,67.3,66.6,55.8,38.3,29.7,17.1$.

HR-ESI-MS (m/z): calcd for $\mathrm{C}_{37} \mathrm{H}_{39} \mathrm{~N}_{2} \mathrm{Cl}_{3} \mathrm{O}_{7} \mathrm{Na}^{+}(\mathrm{M}+\mathrm{Na})^{+}:$753.1694, found: 753.1714 .

$\mathrm{N}$-benzyloxycarbonyl-3-aminopropanyl phenyl (trichloroacetyl carbonylamino)-4-Obenzyl-2,6-dideoxy-3-O-hydroxyl-1- $\beta$-D-galactopyranoside (8)

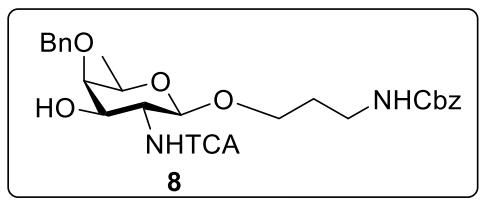

DDQ (0.73 g, $3.2 \mathrm{mmol})$ was added to a stirred solution of $\mathbf{1 5}(1.3 \mathrm{~g}, 1.78 \mathrm{mmol})$ in $\mathrm{CH}_{2} \mathrm{Cl}_{2}$ (91 mL) and $\mathrm{H}_{2} \mathrm{O}(9 \mathrm{~mL})$ at $\mathrm{rt}$, and the reaction mixture was allowed to stir for $4 \mathrm{~h}$. After completion of the reaction, the reaction mixture was quenched with $\mathrm{Et}_{3} \mathrm{~N}$, the reaction mixture was diluted with $\mathrm{CH}_{2} \mathrm{Cl}_{2}(2 \times 100 \mathrm{~mL})$ and washed with saturated sodium bicarbonate solution. Separated organic layer was dried over anhydrous $\mathrm{Na}_{2} \mathrm{SO}_{4}$ and concentrated in vacuo. The crude product was purified by column chromatography on silica gel 2:8 ethyl acetate: pet ether) to obtain $\mathbf{8}$ as a pale yellow viscous liquid $(0.97 \mathrm{~g}, 92 \%)$.

$[\alpha]^{25} \mathrm{D}+3.32\left(c=2.5, \mathrm{CHCl}_{3}\right)$.

IR $\left(\mathrm{cm}^{-1}, \mathrm{CHCl}_{3}\right) v$ 3346, 1698, 1063, 760, 698.

${ }^{1} \mathrm{H}$ NMR (400 MHz, $\left.\mathrm{CDCl}_{3}\right) \delta$ 7.77-7.27 (m, 10H, $\left.\mathrm{ArH}\right), 5.10-5.01\left(\mathrm{~m}, 3 \mathrm{H}, \mathrm{NH}, \mathrm{CH}_{2}\right.$ of Cbz), 4.80-4.77 (m, 2H, $\mathrm{CH}_{2} \mathrm{Ph}$ ), 4.37 (d, $\left.J=8.0 \mathrm{~Hz}, 1 \mathrm{H}, \mathrm{H}-1\right)$, 3.96-3.92 (m, 2H, CH, H-2), 3.803.73 ( m, 1H, H-3), 3.54-3.44 (m, 3H, H-4, CH), 3.37-3.22 (m, $1 \mathrm{H}, \mathrm{CH}), 3.09-2.70(\mathrm{~m}, 1 \mathrm{H}$, H-5), 1.79-1.56 (m, 2H, CH 2 linker), $1.20\left(\mathrm{~d}, J=6.5 \mathrm{~Hz}, 3 \mathrm{H}, \mathrm{CH}_{3}\right)$.

${ }^{13} \mathrm{C} \mathrm{NMR}\left(100 \mathrm{MHz}, \mathrm{CDCl}_{3}\right) \delta 164.0,156.9,138.1,136.9,128.7,128.4,128.2,128.1,100.7$, $92.8,79.3,76.9,76.3,73.7,70.9,66.8,66.5,56.6,37.3,29.8,17.0$.

HR-ESI-MS (m/z): calcd for $\mathrm{C}_{26} \mathrm{H}_{31} \mathrm{~N}_{2} \mathrm{Cl}_{3} \mathrm{O}_{7} \mathrm{Na}^{+}(\mathrm{M}+\mathrm{Na})^{+}:$613.1064, found: 613.1064. 


\section{Phenyl 3- $O$-benzoyl- $\beta$-thio-L-rhamnopyranoside (17)}

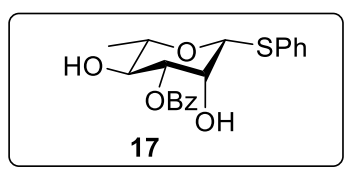

$\mathrm{Me}_{2} \mathrm{SnCl}_{2}(0.103 \mathrm{~g}, 0.47 \mathrm{mmol})$ and DIPEA $(3.26 \mathrm{~mL}, 18.73 \mathrm{mmol})$ were added to a stirred solution of known triol $(2.4 \mathrm{~g}, 9.36 \mathrm{mmol})$ in THF $(48 \mathrm{~mL})$. To this, $\mathrm{BzCl}(1.3 \mathrm{~mL}, 11.24$ mmol) was added, and after $10 \mathrm{~min}$, the reaction mixture was quenched with $2 \mathrm{~N} \mathrm{HCl}(50 \mathrm{~mL})$ and extracted with EtOAc $(2 \times 50 \mathrm{~mL})$. The combined organic layer was washed with aq. $\mathrm{NaHCO}_{3}$, and brine solution, dried over anhydrous $\mathrm{Na}_{2} \mathrm{SO}_{4}$, concentrated in vacuo, and purified using silica gel column chromatography (4:6 ethyl acetate: pet ether) to afford $\mathbf{1 7}$ as a semi solid (3.2 g, 95\%).

$[\alpha]^{25} \mathrm{D}+7.09\left(c=1.0, \mathrm{CHCl}_{3}\right)$.

IR $\left(\mathrm{cm}^{-1}, \mathrm{CHCl}_{3}\right) v 3450,3019,2400,1721,1216,1028,928,765,669 \mathrm{~cm}^{-1}$.

${ }^{1} \mathrm{H}$ NMR $\left(500 \mathrm{MHz}, \mathrm{CDCl}_{3}\right) \delta 8.12(\mathrm{~d}, J=7.4 \mathrm{~Hz}, 2 \mathrm{H}, \mathrm{ArH}), 7.61(\mathrm{t}, J=7.4 \mathrm{~Hz}, 1 \mathrm{H}, \mathrm{ArH})$, $7.54(\mathrm{~d}, J=6.9 \mathrm{~Hz}, 4 \mathrm{H}, \mathrm{ArH}), 7.50-7.31$ (m, 3H, ArH), 5.03 (dd, $J=9.7,2.6 \mathrm{~Hz}, 1 \mathrm{H}, \mathrm{H}-3)$, 4.98 (s, 1H, H-1), 4.44 (d, $J=2.6$ Hz, 1H, H-2), 3.93 (t, $J=9.7$ Hz, 1H, H-4), 3.53-3.50 (m, $1 \mathrm{H}, \mathrm{H}-5), 1.48$ (d, $\left.J=6.2 \mathrm{~Hz}, 3 \mathrm{H}, \mathrm{CH}_{3}\right)$.

${ }^{13} \mathrm{C} \mathrm{NMR}\left(125 \mathrm{MHz}, \mathrm{CDCl}_{3}\right) \delta 166.9,133.8,131.9,130.1,129.3,128.8,127.9,87.2,76.9$, $75.6,71.2,70.8,17.7$.

HR-ESI-MS (m/z): calcd. for $\mathrm{C}_{19} \mathrm{H}_{19} \mathrm{O}_{5} \mathrm{SNa}^{+},(\mathrm{M}+\mathrm{Na})^{+}$383.0924; found 383.0933.

\section{Phenyl 4- $O$-benzoyl-2-azido-2-deoxy-1-thio- $\beta$-L-fucopyranoside (18)}

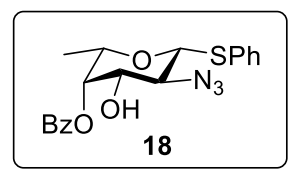

Trifluoromethanesulfonic anhydride $(7.3 \mathrm{~mL}, 43.3 \mathrm{mmol})$ was added to a solution of compound $17(2.6 \mathrm{~g}, 7.21 \mathrm{mmol})$ in pyridine $(7.5 \mathrm{~mL}, 93.0 \mathrm{mmol})$ and $\mathrm{CH}_{2} \mathrm{Cl}_{2}(55 \mathrm{~mL})$ at 0 ${ }^{\circ} \mathrm{C}$. After $1 \mathrm{~h}$ stirring at the same temperature, the reaction mixture was diluted with $\mathrm{CHCl}_{3}$ $(2 \times 100 \mathrm{~mL})$ and was washed with $2 \mathrm{~N} \mathrm{HCl}$, aq. $\mathrm{NaHCO}_{3}$, and brine solution, dried over anhydrous $\mathrm{Na}_{2} \mathrm{SO}_{4}$, filtered and concentrated to give white foam as a product, which was used for the next step without any purification. To a solution of the 2,4-bistriflate in 
acetonitrile $(55 \mathrm{~mL})$ was added tetrabutylammonium azide $(2.05 \mathrm{~g}, 7.21 \mathrm{mmol})$ at $0{ }^{\circ} \mathrm{C}$. After stirring at the same temperature for $1 \mathrm{~h}$, solvent was concentrated from the reaction mixture, and then added a mixture of water $(8 \mathrm{~mL})$ and dichloromethane $(55 \mathrm{~mL})$. The reaction mixture was heated at $80{ }^{\circ} \mathrm{C}$ for $24 \mathrm{~h}$. The reaction was quenched by addition of water and the mixture was extracted with ethyl acetate. The combined organic layer was washed with brine solution, dried over anhydrous $\mathrm{Na}_{2} \mathrm{SO}_{4}$, filtered, and concentrated. The residue was purified by silica gel column chromatography (15\% ethyl acetate : pet ether) to give $\mathbf{1 8}$ as a sticky solid (2.2 g, $76 \%$ over 3 steps).

$[\alpha]^{25} \mathrm{D}-5.19\left(c=1.0, \mathrm{CHCl}_{3}\right)$.

IR $\left(\mathrm{cm}^{-1}, \mathrm{CHCl}_{3}\right) v 3466,2113,1721,1272,1113,1069,92883,753,711 \mathrm{~cm}^{-1}$.

${ }^{1} \mathrm{H}$ NMR (400 MHz, $\left.\mathrm{CDCl}_{3}\right) \delta$ 7.93-7.91 (m, 2H, ArH), 7.73-7.70 (m, 2H, ArH), 7.64-7.59 (m, 1H, ArH), 7.47-7.36 (m, 5H, ArH), 5.38 (dd, $J=3.3,0.7$ Hz, 1H, H-4), 4.47 (d, $J=9.9$ Hz, 1H, H-1), 3.88-3.81 (m, 2H, H- 3, H-5), 3.54 (t, $J=9.9$ Hz, 1H, H-2), 2.47 (d, J = 3.8 Hz, $1 \mathrm{H}, \mathrm{OH}), 1.30\left(\mathrm{~d}, J=6.4 \mathrm{~Hz}, 3 \mathrm{H}, \mathrm{CH}_{3}\right)$.

${ }^{13} \mathrm{C}$ NMR $\left(100 \mathrm{MHz}, \mathrm{CDCl}_{3}\right) \delta 168.9,134.6,133.8,130.8,130.1,129.3,129.2,128.7,85.7$, $73.9,73.5,72.6,62.5,17.1$.

HR-ESI-MS (m/z): calcd. for $\mathrm{C}_{19} \mathrm{H}_{19} \mathrm{~N}_{3} \mathrm{O}_{4} \mathrm{SNa}^{+},(\mathrm{M}+\mathrm{Na})^{+}$408.0988; found 408.0998.

\section{Phenyl 2-azido-2-deoxy-3- $O$-allyl-4- $O$-benzoyl-1-thio- $\beta$-L-fucopyranoside (7a)}

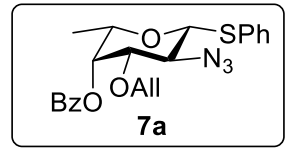

AllylBr (0.1 mL, $1.24 \mathrm{mmol})$ was added to a stirred solution of $18(0.40 \mathrm{~g}, 1.03 \mathrm{mmol})$ in DMF (5.4 mL) at $-15^{\circ} \mathrm{C}$, after $5 \mathrm{~min} \mathrm{NaH}(40 \mathrm{mg}, 1.64 \mathrm{mmol}$ ) was added portion wise and the reaction mixture was allowed to stir for $1 \mathrm{~h}$. After completion of the starting material, the reaction mixture was diluted with EtOAc and washed with brine solution. Separated organic layer was dried over anhydrous $\mathrm{Na}_{2} \mathrm{SO}_{4}$ and concentrated in vacuo. The crude product was purified by column chromatography on silica gel (1:9 ethyl acetate : pet ether) to obtain $\mathbf{7 a}$ as pale yellow liquid $(0.375 \mathrm{~g}, 85 \%)$.

$[\alpha]^{25} \mathrm{D}+61.12\left(c=0.6, \mathrm{CHCl}_{3}\right)$. 
IR $\left(\mathrm{cm}^{-1}, \mathrm{CHCl}_{3}\right) v 2933,2114,1722,1269,1061,752,713$.

${ }^{1} \mathrm{H}$ NMR (400 MHz, $\left.\mathrm{CDCl}_{3}\right) \delta$ 8.14-8.12 (m, 2H, ArH), 7.89-7.87 (m, 2H, ArH), 7.73-7.63 (m, 2H, ArH), 7.61-7.26 (m, 4H, ArH), 5.89-5.82 (m, 1H, CH), 5.48 (d, J = 2.4 Hz, 1H, H4), $5.26(\mathrm{dd}, J=17.2 \mathrm{~Hz}, 1.6 \mathrm{~Hz}, 1 \mathrm{H}, \mathrm{CH}), 5.16(\mathrm{dd}, J=10.4 \mathrm{~Hz}, 1.2 \mathrm{~Hz}, 1 \mathrm{H}, \mathrm{CH}), 4.43(\mathrm{~d}, J$ $=9.6 \mathrm{~Hz}, 1 \mathrm{H}, \mathrm{H}-1), 4.20(\mathrm{dd}, J=12.4 \mathrm{~Hz}, 5.6 \mathrm{~Hz}, 1 \mathrm{H}, \mathrm{CH}), 4.04(\mathrm{dd}, J=12.4 \mathrm{~Hz}, 6.4 \mathrm{~Hz}$, $1 \mathrm{H}, \mathrm{CH}), 3.81-3.79$ (m, 1H, H-5), 3.60-3.52 (m, 2H, H-3, H-2), 1.29 (d, J = 6.4 Hz, 3H, $\left.\mathrm{CH}_{3}\right)$.

${ }^{13} \mathrm{C} \mathrm{NMR}\left(100 \mathrm{MHz}, \mathrm{CDCl}_{3}\right) \delta 172.3,166.1,135.0,133.9,133.5,130.4,130.1,129.5,129.1$, $128.7,128.6,128.5,118.4,85.3,79.6,73.7,70.9,69.2,60.7,17.1$.

HR-ESI-MS (m/z): calcd. for $\mathrm{C}_{22} \mathrm{H}_{23} \mathrm{~N}_{3} \mathrm{O}_{4} \mathrm{SNa}^{+},(\mathrm{M}+\mathrm{Na})^{+} 448.1301$; found, 448.1305.

\section{Phenyl 4,6-O-benzylidene-1-thio- $\beta$-D-glucopyranoside (19)}

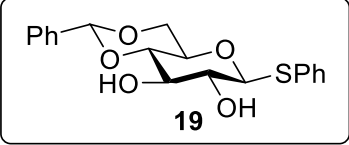

Phenyl 4,6- $O$-benzylidene-1-thio- $\beta$-D-glucopyranoside 19 was prepared following a procedure reported by Amy Howell and co-workers. ${ }^{1}$ Compound 19 was synthesised from commercially available $\mathrm{D}$-glucose, via per- $O$-acetylation followed by $\mathrm{BF}_{3} . \mathrm{OEt}_{2}$ mediated anomeric acetate displacement by thiphenol, deacetylation and 4,6-O-benzylidation. The characterization data of compound $\mathbf{1 9}$ matches perfectly with the reported data.

${ }^{1} \mathrm{H}$ NMR (400 MHz, $\left.\mathrm{CDCl}_{3}\right) \delta$ 7.55-7.47 (m, 4H, ArH), 7.37-7.26 (m, 6H, ArH), $5.53(\mathrm{~s}, 1 \mathrm{H}$, $\mathrm{PhCHH}), 4.63$ (d, $J=9.73 \mathrm{~Hz}, 1 \mathrm{H}, \mathrm{H}-1), 4.38$ (dt, $J=10.5 \mathrm{~Hz}, 2.11 \mathrm{~Hz} 1 \mathrm{H}, \mathrm{H}-5), 3.84$ (t, $J=$ $8.64 \mathrm{~Hz}, 1 \mathrm{H}, \mathrm{H}-3), 3.78$ (t, $J=9.90 \mathrm{~Hz}, 1 \mathrm{H}, \mathrm{H}-6), 3.52-3-3.50$ (m, 2H, H-4, H-6'), 3.46 (t, $J=$ $9.21 \mathrm{~Hz}, 1 \mathrm{H}, \mathrm{H}-2), 2.99$ (bs, 1H, OH), 2.82 (bs, 1H, OH).

Ref 1. Raju, R.; Castillo, B. F.; Richardson, S. K.; Thakur, M.; Severins, R.; Kronenberg, M.; Howell, A. R. Synthesis and evaluation of 3"- and 4"-deoxy and -fluoro analogs of the immunostimulatory glycolipid, KRN7000. Bioorg. Med. Chem. Lett. 2009, 19, 4122-4125. 
${ }^{13} \mathrm{C} \mathrm{NMR}\left(100 \mathrm{MHz}, \mathrm{CDCl}_{3}\right) \delta 136.8,133.1,131.3,129.4,129.2,128.5,128.4,126.3,101.9$, $88.6,80.2,74.6,72.6,70.5,68.6$.

HR-ESI-MS (m/z): calcd. for $\mathrm{C}_{19} \mathrm{H}_{20} \mathrm{O}_{5} \mathrm{SNa}^{+},(\mathrm{M}+\mathrm{Na})^{+}$383.0923; found, 383.0924 .

Phenyl 4,6- $O$-benzylidene-3- $O$-benzyl-1-thio- $\beta$-D-glucopyranoside (20)

$$
\text { SPh }
$$

Known diol $19^{52}(1.2 \mathrm{~g}, 3.3 \mathrm{mmol})$ was dissolved in dry toluene $(23 \mathrm{~mL})$, to this stirred solution $\mathrm{Bu}_{2} \mathrm{SnO}(1.23 \mathrm{~g}, 4.98 \mathrm{mmol})$ was added and reaction mixture was kept at $110{ }^{\circ} \mathrm{C}$ for $8 \mathrm{~h}$. After completion of the reaction, solvent was removed under reduced pressure, crude compound was kept for vacum $8 \mathrm{~h}$.

Above crude was dissolved in dry toluene $(12 \mathrm{~mL})$, and kept at $60{ }^{\circ} \mathrm{C}$ under reflux condition. At this temperature $\mathrm{TBAB}(1.6 \mathrm{~g}, 4.98 \mathrm{mmol})$ followed by $\mathrm{BnBr}(0.6 \mathrm{~mL}, 4.98$ mmol) were added and reaction mixture was kept stirring at the same temperature. After $6 \mathrm{~h}$ (confirmed by TLC) organic layer was washed with brine solution several times, dried over anhydrous $\mathrm{Na}_{2} \mathrm{SO}_{4}$, organic layer was concentrated, and purified by column chromatography on silica gel (15\% ethyl acetate : pet ether) to obtain $\mathbf{2 0}$ as a sticky solid (1.08 g, 72\%).

$[\alpha]^{25} \mathrm{D}-48.17\left(c=0.3, \mathrm{CHCl}_{3}\right)$.

IR $\left(\mathrm{cm}^{-1}, \mathrm{CHCl}_{3}\right) v 3747,3369,2330,1072,791,750,668$.

${ }^{1} \mathrm{H}$ NMR (500 MHz, $\left.\mathrm{CDCl}_{3}\right) \delta$ 7.58-7.57 (m, 2H, ArH), 7.56-7.50 (m, 2H, ArH), 7.43-7.39 (m, 5H, ArH), 7.36-7.28 (m, 6H, ArH), $5.60(\mathrm{~s}, 1 \mathrm{H}, \mathrm{PhCHH}), 4.98(\mathrm{~d}, J=11.5 \mathrm{~Hz}, 1 \mathrm{H}$, $\left.\mathrm{CH}_{2} \mathrm{Ph}\right), 4.82\left(\mathrm{~d}, J=11.5 \mathrm{~Hz}, 1 \mathrm{H}, \mathrm{CH}_{2} \mathrm{Ph}\right), 4.66(\mathrm{~d}, J=10.0 \mathrm{~Hz}, 1 \mathrm{H}, \mathrm{H}-1), 4.42(\mathrm{dd}, J=10.5$ Hz, 5.0 Hz 1H, H-4), 3.82 (t, $J=10.0$ Hz, 1H, H-3) 3.74-3-3.67 (m, 2H, H-6a, H-6b), 3.573.52 (m, 2H, H-2, H-5), 2.62 (bs, 1H, OH).

${ }^{13} \mathrm{C} \mathrm{NMR}\left(125 \mathrm{MHz}, \mathrm{CDCl}_{3}\right) \delta 138.3,137.4,133.4,131.5,129.3,129.2,128.7,128.6,128.5$, $128.3,128.1,126.2,101.4,88.6,81.8,81.3,75.0,72.4,70.9,68.8$.

HR-ESI-MS (m/z): calcd. for $\mathrm{C}_{26} \mathrm{H}_{26} \mathrm{O}_{5} \mathrm{SNa}^{+},(\mathrm{M}+\mathrm{Na})^{+} 473.1393$; found, 473.1397 .

Phenyl 4,6- $O$-benzylidene-3- $O$-benzyl-2- $O$-benzoyl-1-thio- $\beta$-D-glucopyranoside (6) 


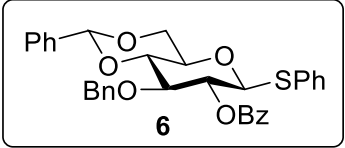

Compound 20 (0.56 gm, $1.24 \mathrm{mmol})$ was dissolved in $\mathrm{CH}_{2} \mathrm{Cl}_{2}(7 \mathrm{~mL})$ and pyridine $(0.4 \mathrm{~mL}$, $3.72 \mathrm{mmol})$ and $\mathrm{BzCl}(0.43,3.72 \mathrm{mmol})$ were added slowly. After stirring the reaction mixture at room temperature for $1 \mathrm{~h}$, it was diluted with $\mathrm{CH}_{2} \mathrm{Cl}_{2}(2 \times 20 \mathrm{~mL})$ and washed with brine. Separated organic layer was dried over anhydrous $\mathrm{Na}_{2} \mathrm{SO}_{4}$, concentrated and purified by column chromatography on silica gel (1:9 ethyl acetate : pet ether) to afford $\mathbf{6}$ as white solid $(0.61 \mathrm{~g}, 90 \%)$.

$[\alpha]^{25} \mathrm{D}+18.46\left(c=0.6, \mathrm{CHCl}_{3}\right) ;$ M.P. $150{ }^{\circ} \mathrm{C}$.

IR $\left(\mathrm{cm}^{-1}, \mathrm{CHCl}_{3}\right) v 2867,2329,1723,1270,1099,751$.

${ }^{1} \mathrm{H}$ NMR (500 MHz, $\left.\mathrm{CDCl}_{3}\right) \delta 8.03(\mathrm{~d}, J=8.0 \mathrm{~Hz}, 2 \mathrm{H}, \mathrm{ArH}), 7.63-7.05$ (m, 18H, ArH), 5.61 (s, 1H, PhCHH), 5.30 (t, $J=9.5 \mathrm{~Hz}, 1 \mathrm{H}, \mathrm{H}-2), 4.86$ (d, $J=10.0 \mathrm{~Hz}, 1 \mathrm{H}, \mathrm{H}-1), 4.82$ (d, $J=$ $12.0 \mathrm{~Hz}, 1 \mathrm{H}, \mathrm{CH}_{2} \mathrm{Ph}$ ), 4.67 (d, $J=11.5 \mathrm{~Hz}, 1 \mathrm{H}, \mathrm{CH}_{2} \mathrm{Ph}$ ), 4.43 (dd, $J=10.5 \mathrm{~Hz}, 4.5 \mathrm{~Hz}, 1 \mathrm{H}$, H-6a), 3.92-3.81 (m, 3H, H-3, H-4, H-6b), 3.60-3.56 (m, 1H, H-5).

${ }^{13} \mathrm{C}$ NMR $\left(125 \mathrm{MHz}, \mathrm{CDCl}_{3}\right) \delta 165.2,137.8,137.3,133.9,133.4,133.1,132.4,130.4,130.1$, $129.9,129.2$, 129.1, 128.7, 128.6, 128.5, 128.4, 128.39. 128.30, 127.8, 126.2, 101.5, 87.2, $81.6,79.5,74.4,72.2,70.8,68.8$.

HR-ESI-MS (m/z): calcd. for $\mathrm{C}_{33} \mathrm{H}_{30} \mathrm{O}_{6} \mathrm{SNa}^{+},(\mathrm{M}+\mathrm{Na})^{+}$577.1655; found, 577.1654.

Phenyl 4- $O$-hydroxyl-3,6-di- $O$-benzyl-2- $O$-benzoyl-1-thio- $\beta$-D-glucopyranoside (21)

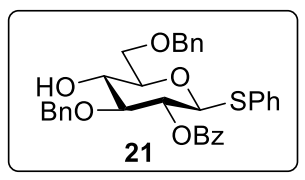

$\mathrm{Et}_{3} \mathrm{SiH}(0.7 \mathrm{~mL}, 4.50 \mathrm{mmol})$ and TFA $(0.4 \mathrm{~mL}, 4.50 \mathrm{mmol})$ were added sequentially to a solution of $6(0.5 \mathrm{~g}, 0.9 \mathrm{mmol})$ in $\mathrm{CH}_{2} \mathrm{Cl}_{2}(6 \mathrm{~mL})$ at $0{ }^{\circ} \mathrm{C}$. After complete consumption of starting material (as monitored by TLC), $\mathrm{Et}_{3} \mathrm{~N}(1 \mathrm{~mL})$ was added to the reaction mixture. Solvent were removed under reduced pressure and the obtained crude product was purified by column chromatography on silica gel (25\% ethyl acetate: pet ether) to afford $\mathbf{2 1}$ as a sticky foam $(0.40 \mathrm{~g}, 82 \%)$.

$[\alpha]^{25} \mathrm{D}+0.40\left(c=0.4, \mathrm{CHCl}_{3}\right)$. 
IR $\left(\mathrm{cm}^{-1}, \mathrm{CHCl}_{3}\right) v 3452,2336,1721,1270,1069,750$.

${ }^{1} \mathrm{H}$ NMR (400 MHz, $\left.\mathrm{CDCl}_{3}\right) \delta$ 8.15-8.13 (m, 2H, ArH), 7.70-7.26 (m, 19H, ArH), 5.35 (t, $J=$ $9.2 \mathrm{~Hz}, 1 \mathrm{H}, \mathrm{H}-2), 4.89$ (d, $J=10.0 \mathrm{~Hz}, 1 \mathrm{H}, \mathrm{H}-1), 4.81-4.72\left(\mathrm{~m}, 2 \mathrm{H}, \mathrm{CH}_{2} \mathrm{Ph}\right), 4.71-4.64$ (m, 2H, $\mathrm{CH}_{2} \mathrm{Ph}$ ), 3.91-3.76 (m, 4H, H-6a, H-6b, H-4, H-3), 3.71-3.67 (m, 1H, H-5).

${ }^{13} \mathrm{C} \mathrm{NMR}\left(100 \mathrm{MHz}, \mathrm{CDCl}_{3}\right) \delta 165.4,138.0,133.5,133.2,132.6,130.1,129.0,128.7,128.6$, $128.2,128.07,128.03,127.9,86.6,83.9,78.6,74.9,73.9,72.3,72.0,70.5$.

HR-ESI-MS (m/z): calcd. For $\mathrm{C}_{33} \mathrm{H}_{32} \mathrm{O}_{6} \mathrm{SNa}^{+},(\mathrm{M}+\mathrm{Na})^{+}$579.1812; found, 579.1819 .

Phenyl 3,4,6-tri- $O$-benzyl-2- $O$-benzoyl-1-thio- $\beta$-D-glucopyranoside (22)

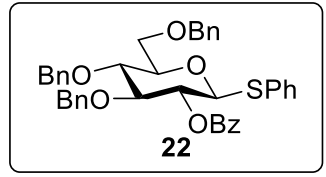

$\operatorname{BnBr}(0.02 \mathrm{~mL}, 0.122 \mathrm{mmol})$ was added to a stirred solution of 21 (57 $\mathrm{mg}, 0.10 \mathrm{mmol})$ in DMF $(1 \mathrm{~mL})$ at $0{ }^{\circ} \mathrm{C}$. After $5 \mathrm{~min} \mathrm{NaH}(40 \mathrm{mg}, 0.16 \mathrm{mmol})$ was added portion wise and the reaction mixture was allowed to stir for $1 \mathrm{~h}$. After complete consumtion of the starting material, the reaction mixture was diluted with EtOAc and washed with water. Separated organic layer was dried over anhydrous $\mathrm{Na}_{2} \mathrm{SO}_{4}$ and concentrated in vacuo. The crude product was purified by column chromatography on silica gel (1:9 ethyl acetate: pet ether) to obtain 22 as a viscous liquid (64 mg, 95\%).

$[\alpha]^{25} \mathrm{D}+11.75\left(c=0.2, \mathrm{CHCl}_{3}\right)$.

IR $\left(\mathrm{cm}^{-1}, \mathrm{CHCl}_{3}\right) v 2342,1730,1253,751$.

${ }^{1} \mathrm{H}$ NMR (400 MHz, $\left.\mathrm{CDCl}_{3}\right) \delta$ 8.04-8.02 (m, 2H, ArH), 7.59-7.11 (m, 23H, ArH), 5.28 (t, $J=$ 10.0 Hz, 1H, H-2), 4.82-4.53 (m, 7H), 3.86-3.71 (m, 4H, H-6a, H-6b, H-3, H-4), 3.36-3.30 (m, 1H, H-5).

${ }^{13} \mathrm{C}$ NMR (100 MHz, $\left.\mathrm{CDCl}_{3}\right) \delta 165.4,138.4,138.1,137.9,133.4,133.1,132.7,130.1,130.0$, $129.0,128.7,128.6,128.5,128.4,128.3,128.2$, 128.0, 127.98, 127.91,127.8, 86.3, 84.5, 79.7, 78.0, 77.5, 76.9, 75.5, 75.3, 73.7, 72.7, 69.2.

HR-ESI-MS (m/z): calcd. for $\mathrm{C}_{40} \mathrm{H}_{38} \mathrm{O}_{6} \mathrm{SK}^{+},(\mathrm{M}+\mathrm{K})^{+}$685.2021; found, 685.2029. 
$N$-benzyloxycarbonyl-3-aminopropanyl- $O$-(2-azido-2-deoxy-4- $O$-benzoyl-3- $O$-allyl- $\alpha$-Lfucopyranosyl)-( $1 \rightarrow 3)$-2-(trichloroacetylcarbonylamino)-2-deoxy-4- $O$-benzyl- $\beta$-Dfucopyranoside (23)

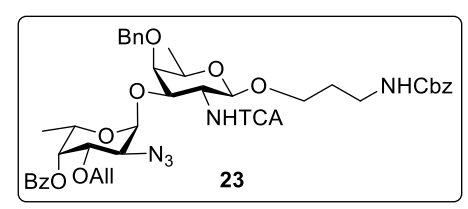

TMSOTf ( $5 \mu \mathrm{L}, 0.03 \mathrm{mmol}$ ) was added dropwise to a solution of donor 18 (55 $\mathrm{mg}, 0.129$ $\mathrm{mmol}$ ), acceptor 8 (40 mg, $0.06 \mathrm{mmol}), 3 \AA \mathrm{MS}(0.23 \mathrm{~g})$ and NIS (60 mg, $0.25 \mathrm{mmol})$ in $\mathrm{CH}_{2} \mathrm{Cl}_{2}(1.85 \mathrm{~mL})$ at $0{ }^{\circ} \mathrm{C}$. After $30 \mathrm{~min}$ the reaction mixture was quenched with $\mathrm{Et}_{3} \mathrm{~N}$ diluted with $\mathrm{CH}_{2} \mathrm{Cl}_{2}$, filtered through Celite, organic layer was washed with aq. $\mathrm{Na}_{2} \mathrm{~S}_{2} \mathrm{O}_{3}$ solution, dried over anhydrous $\mathrm{Na}_{2} \mathrm{SO}_{4}$ and concentrated. The residue was purified by chromatography on silica gel ( $25 \%$ ethyl acetate: pet ether) to give the desired product $\mathbf{2 3}$ as viscous liquid $(62 \%, 35 \mathrm{mg})$.

$[\alpha]^{25} \mathrm{D}-7.86\left(c=0.2, \mathrm{CHCl}_{3}\right)$.

IR $\left(\mathrm{cm}^{-1}, \mathrm{CHCl}_{3}\right) v 3848,3746,2933,2339,1715,1216,1047,789,753$.

${ }^{1} \mathrm{H}$ NMR (400 MHz, $\left.\mathrm{CDCl}_{3}\right) \delta$ 8.03-8.02 (m, 2H, ArH), 7.58-7.26 (m, 13H, ArH), 5.84-5.79 (m, 1H, CH), 5.32 (bs, 1H, H-4'), 5.22 (d, J=1.2 Hz, 1H, CH), 5.18 (d, J=1.2 Hz, 1H, CH), 5.13-5.06 (m, 2H, NH, CH Ph), 5.03 (d, J = 3.2 Hz, 1H, H-1'), 4.89-4.84 (m, 2H, H-1, CH $\mathrm{Ph}$ ), 4.74 (d, $J=12.0 \mathrm{~Hz}, 1 \mathrm{H}, \mathrm{CH} \mathrm{Ph}), 4.27-4.24$ (m, 1H, H-3), 4.19-4.11 (m, 1H, CH), 3.993.91 (m, 2H), 3.80-3.72 (m, 3H, H-2', H-3', CH), 3.66-3.63 (m, 2H), 3.59-3.51 (m, 3H, H-4, $\mathrm{H}-5, \mathrm{CH}), 3.35-3.33$ (m, 1H, CH), 3.27-3.22 (m, 1H, CH), 1.77-1.63 (m, 3H), 1.31 (d, J=6.4 $\left.\mathrm{Hz}, 3 \mathrm{H}, \mathrm{CH}_{3}\right), 1.08\left(\mathrm{~d}, J=6.4 \mathrm{~Hz}, 3 \mathrm{H}, \mathrm{CH}_{3}\right)$.

${ }^{13} \mathrm{C} \mathrm{NMR}\left(125 \mathrm{MHz}, \mathrm{CDCl}_{3}\right) \delta 166.1,162.4,156.7,138.6,134.0,133.5,130.0,129.9,129.7$, 129.2, 128.7, 128.6, 128.2, 128.0, 127.5, 117.9, 99.6, 92.8, 78.5, 75.6, 74.8, 74.7, 71.0, 70.5, $69.8,67.5,66.7,66.2,60.0,55.8,38.4,29.8,17.3,16.6$.

HR-ESI-MS (m/z): calcd. for $\mathrm{C}_{42} \mathrm{H}_{48} \mathrm{Cl}_{3} \mathrm{~N}_{5} \mathrm{O}_{11} \mathrm{Na}^{+},(\mathrm{M}+\mathrm{Na})^{+}$928.2289; found, 928.2287.

$\mathrm{N}$-benzyloxycarbonyl-3-aminopropanyl-O $O$-(2-azido-2-deoxy-4- $O$-benzoyl-3- $O$-hydroxy$\alpha$-L-fucopyranosyl)-( $1 \rightarrow 3$ )-2-(trichloroacetyl carbonylamino)-2-deoxy-4- $O$-benzyl- $\beta$-Dfucopyranoside (24) 


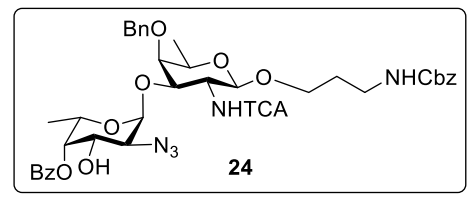

To a solution of disaccharide 23 (30 mg, $0.03 \mathrm{mmol})$ in dry $\mathrm{MeOH}(1.2 \mathrm{ml}), \mathrm{PdCl}_{2}(10 \mathrm{mg}$,) was added under nitrogen atmosphere. The mixture was stirred for $3 \mathrm{~h}$, and monitored by TLC. The mixture was filtered on celite bed, the filtrate was concentrated on rotory evaporator first and the residue was purified by column chromatography on silica gel (4:6 ethyl acetate: pet ether) to give $\mathbf{2 4}$ as a sticky solid (21 $\mathrm{mg}, 82 \%$ ).

$[\alpha]^{25} \mathrm{D}-19.03\left(c=0.03, \mathrm{CHCl}_{3}\right)$.

IR $\left(\mathrm{cm}^{-1}, \mathrm{CHCl}_{3}\right) v 3830,3748,2329,1664,809,751$.

${ }^{1} \mathrm{H}$ NMR (400 MHz, $\left.\mathrm{CDCl}_{3}\right) \delta$ 8.05-8.04 (m, 2H, ArH), 7.61-7.26 (m, 13H, ArH), 5.15 (bs, $\left.1 \mathrm{H}, \mathrm{H}-4^{\prime}\right), 5.07-5.05$ (m, 2H, $\mathrm{CH}_{2}$ of Cbz), 5.04 (d, $\left.J=3.6 \mathrm{~Hz}, 1 \mathrm{H}, \mathrm{H}-1^{\prime}\right), 4.87$ (d, $J=12.4$ $\mathrm{Hz}, 1 \mathrm{H}, \mathrm{CH} \mathrm{Ph}), 4.83$ (d, J=8.0 Hz, 1H, H-1), 4.72 (d, J=12.0 Hz, 1H, CH Ph), 4.29-4.26 $(\mathrm{m}, 1 \mathrm{H}), 4.04-3.93(\mathrm{~m}, 4 \mathrm{H}), 3.68-3.49(\mathrm{~m}, 4 \mathrm{H}), 3.36-3.33(\mathrm{~m}, 1 \mathrm{H}, \mathrm{CH}), 3.27-3.21(\mathrm{~m}, 1 \mathrm{H}$, $\mathrm{CH}), 1.80-1.73(\mathrm{~m}, 5 \mathrm{H}), 1.32\left(\mathrm{~d}, J=6.4 \mathrm{~Hz}, 3 \mathrm{H}, \mathrm{CH}_{3}\right), 1.08\left(\mathrm{~d}, J=6.5 \mathrm{~Hz}, 3 \mathrm{H}, \mathrm{CH}_{3}\right)$.

${ }^{13} \mathrm{C} \mathrm{NMR}\left(100 \mathrm{MHz}, \mathrm{CDCl}_{3}\right) \delta 167.1,162.5,156.7,138.5,136.9,133.7,130.0,129.9,129.4$, 128.7, 128.66, 128.62, 128.4, 128.2, 127.9, 127.6, 99.6, 92.7, 79.5, 78.2, 76.9, 75.5, 73.9, $71.0,68.1,67.4,66.7,66.2,61.0,55.6,38.2,29.8,17.3,17.0,16.4$.

HR-ESI-MS (m/z): calcd. for $\mathrm{C}_{39} \mathrm{H}_{44} \mathrm{Cl}_{3} \mathrm{~N}_{5} \mathrm{O}_{11} \mathrm{Na}^{+},(\mathrm{M}+\mathrm{Na})^{+}$888.1995; found, 888.1976.

\section{Phenyl 3- $O$-acetyl-2-azido-2-deoxy-4- $O$-benzoyl-1-thio- $\beta$-L-fucopyranoside (7b)}

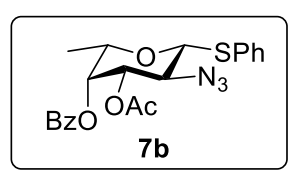

Compound $18(0.73 \mathrm{~g}, 1.90 \mathrm{mmol})$ was dissolved in pyridine $(0.5 \mathrm{ml})$, to this stirred solution, $\mathrm{AcCl}(0.2 \mathrm{ml})$ was added in dropwise manner at $0{ }^{\circ} \mathrm{C}$ and reaction mixture was kept at stirring for $30 \mathrm{~min}$. After completion of the reaction (confirmed by TLC) organic layer was washed with $1 \mathrm{~N} \mathrm{HCl}$ and brine solution, dried over anhydrous $\mathrm{Na}_{2} \mathrm{SO}_{4}$, organic layer was concentrated, and purified by column chromatography on silica gel (1:9 ethyl acetate: pet ether) to obtain $\mathbf{7 b}$ as a sticky solid $(0.77 \mathrm{~g}, 92 \%)$. 
$[\alpha]^{25} \mathrm{D}-3.20\left(c=1.2, \mathrm{CHCl}_{3}\right)$.

IR $\left(\mathrm{cm}^{-1}, \mathrm{CHCl}_{3}\right) v 2932,2113,1752,1727,1268,1084,754$.

${ }^{1} \mathrm{H}$ NMR $\left(500 \mathrm{MHz}, \mathrm{CDCl}_{3}\right) \delta$ 7.89-7.87 (m, 2H, ArH), 7.73-7.62 (m, 3H, ArH), 7.47-7.26 (m, 5H, ArH), 5.45-5.44 (m, 1H, H-4), 4.96 (dd, $J=10.2 \mathrm{~Hz}, 3.2 \mathrm{~Hz}, 1 \mathrm{H}, \mathrm{H}-3), 4.51$ (d, $J=$ $10.0 \mathrm{~Hz}, 1 \mathrm{H}, \mathrm{H}-1), 3.92-3.91$ (m, 1H, H-5), 3.65 (t, $J=10.0 \mathrm{~Hz}, 1 \mathrm{H}, \mathrm{H}-2), 1.97$ (s, 3H, $\left.\mathrm{COCH}_{3}\right), 1.28\left(\mathrm{~d}, J=6.5 \mathrm{~Hz}, 3 \mathrm{H}, \mathrm{CH}_{3}\right)$.

${ }^{13} \mathrm{C}$ NMR $\left(125 \mathrm{MHz}, \mathrm{CDCl}_{3}\right) \delta 170.1,165.9,135.0,134.0,133.7,130.4,130.3,130.1,129.4$, $129.3,129.2,128.9,128.8,128.7,85.6,73.8,73.7,70.3,59.3,20.9,16.9$.

HR-ESI-MS (m/z): calcd for $\mathrm{C}_{21} \mathrm{H}_{21} \mathrm{~N}_{3} \mathrm{O}_{5} \mathrm{SNa}^{+},(\mathrm{M}+\mathrm{Na})^{+} 450.1094$; found 450.1093 .

$N$-benzyloxycarbonyl-3-aminopropanyl-O-(3-O-acetyl 2-azido-2-deoxy-4- $O$-benzoyl-)$(1 \rightarrow 3)$ - $\alpha$-L-fucopyranosyl-2-(trichloroacetyl carbonylamino)-2-deoxy-4- $O$-benzyl- $\beta$-Dfucopyranoside (25)

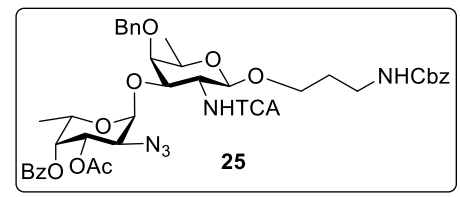

Thioglycoside donor $7 \mathbf{b}(0.52 \mathrm{~g}, 1.21 \mathrm{mmol})$ and acceptor 8 (0.58 g, $0.97 \mathrm{mmol})$ were azeotroped with toluene and dissolved in anhydrous $\mathrm{CH}_{2} \mathrm{Cl}_{2}(18 \mathrm{~mL})$. Molecular sieves $(3 \AA$, $1.2 \mathrm{~g}$ ) were added, and the mixture was stirred at room temperature for 30 minutes under nitrogen. NIS $(0.55 \mathrm{~g}, 2.42 \mathrm{mmol})$ was added then the mixture was cooled to $0{ }^{\circ} \mathrm{C}$ and TMSOTf (43 $\mu \mathrm{L}, 0.242 \mathrm{mmol}$ ) were added dropwise. The mixture was neutralized with $\mathrm{Et}_{3} \mathrm{~N}$, filtered through a Celite bed using $\mathrm{CH}_{2} \mathrm{Cl}_{2}$. The organic layer was washed with aq. $\mathrm{Na}_{2} \mathrm{~S}_{2} \mathrm{O}_{3}$ solution, separated organic layer was dried over anhydrous $\mathrm{Na}_{2} \mathrm{SO}_{4}$, and concentrated in vacuo purified by column chromatography on Flash silica gel (3:7 ethyl acetate: pet ether) afforded disaccharide 25 as white foam $(0.84 \mathrm{~g}, 94 \%)$.

$[\alpha]^{25} \mathrm{D}-40.78\left(c=0.13, \mathrm{CHCl}_{3}\right)$.

IR $\left(\mathrm{cm}^{-1}, \mathrm{CHCl}_{3}\right) v 2113,1698,1532,1269,821,755$.

${ }^{1} \mathrm{H}$ NMR (400 MHz, $\left.\mathrm{CDCl}_{3}\right) \delta$ 8.07-8.04 (m, 2H, ArH), 7.63-7.16 (m, 13H, ArH), 5.37-5.34 (m, 2H, H-4', H-3'), 5.14-5.07 (m, 4H, H-1', NH, $\mathrm{CH}_{2}$ of Cbz), 4.91 (d, J=8.0 Hz, 1H, H-1), 4.88-4.79 (m, 2H, CHHPh), 4.39-4.37 (m, 1H, H-3), 3.99-3.92 (m, 3H, H-2, CH), 3.80-3.78 
(m, 1H, H-2'), 3.66-3.55 (m, 3H, H-4, H-5, CH), 3.35-3.32 (m, 1H, CH), 3.27-3.24 (m, 1H, $\mathrm{CH}), 2.08\left(\mathrm{~s}, 3 \mathrm{H}, \mathrm{COCH}_{3}\right), 1.77-1.76(\mathrm{~m}, 3 \mathrm{H}), 1.35\left(\mathrm{~d}, J=6.0 \mathrm{~Hz}, 3 \mathrm{H}, \mathrm{CH}_{3}\right), 1.05(\mathrm{~d}, J=6.0$ $\left.\mathrm{Hz}, 3 \mathrm{H}, \mathrm{CH}_{3}\right)$.

${ }^{13} \mathrm{C} \mathrm{NMR}\left(100 \mathrm{MHz}, \mathrm{CDCl}_{3}\right) \delta 169.9,166.0,162.5,156.7,138.2,136.9,133.7,130.0,129.4$, 129.2, 128.84, 128.82, 128.7, 128.2, 128.1, 127.7, 99.6, 99.1, 92.7, 78.9, 78.2, 76.9, 75.2, $71.1,71.0,69.3,67.5,66.7,65.9,58.3,56.0,38.4,29.8,20.9,17.5,16.3$.

HR-ESI-MS (m/z): calcd. for $\mathrm{C}_{41} \mathrm{H}_{46} \mathrm{Cl}_{3} \mathrm{~N}_{5} \mathrm{O}_{12} \mathrm{Na}^{+},(\mathrm{M}+\mathrm{Na})^{+}$930.2082; found, 930.2078.

$N$-benzyloxycarbonyl-3-aminopropanyl-O-(2-azido-2-deoxy-3,4-di-ol)-(1 $\rightarrow 3$ )- $\alpha$-L-

fucopyranosyl-2-(trichloroacetylcarbonylamino)-2-deoxy-4- $O$-benzyl- $\beta$-D-

fucopyranoside (5)

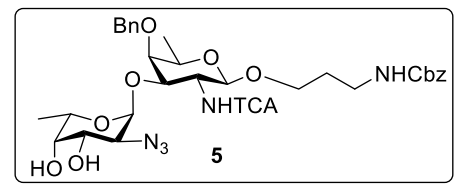

$\mathrm{NaOMe}(0.22 \mathrm{~g})$ was added to a stirred solution of $25(0.55 \mathrm{~g}, 0.60 \mathrm{mmol})$ in $\mathrm{MeOH}(9.0$ $\mathrm{mL})$. After 30 minutes reaction mixture was neutralized with Amberlite IR $120\left(\mathrm{H}^{+}\right)(0.13 \mathrm{~g})$ resin. The reaction mixture was filtered and concentrated under reduced pressure purified by column chromatography on silica gel (4:6 ethyl acetate: pet ether) to afford desired compound $\mathbf{5}$ as viscous liquid $(0.38 \mathrm{~g}, 82 \%)$.

$[\alpha]^{25} \mathrm{D}-83.44\left(c=0.05, \mathrm{CHCl}_{3}\right)$.

IR $\left(\mathrm{cm}^{-1}, \mathrm{CHCl}_{3}\right) v 3369,2113,1701,1101,791,668$.

${ }^{1} \mathrm{H}$ NMR (400 MHz, $\left.\mathrm{CDCl}_{3}\right) \delta$ 7.48-7.26 (m, 10H, ArH), 5.19-5.16 (m, 1H, NH), 5.11-5.06 (m, 2H, $\mathrm{CH}_{2}$ of Cbz), 4.95 (d, $J=3.6 \mathrm{~Hz}, 1 \mathrm{H}, \mathrm{H}-1$ '), 4.79-4.72 (m, 2H, CHHPh), 4.21-4.18 $(\mathrm{m}, 1 \mathrm{H}), 4.00-3.91(\mathrm{~m}, 2 \mathrm{H}), 3.87-3.78(\mathrm{~m}, 2 \mathrm{H}), 3.65-3.47(\mathrm{~m}, 5 \mathrm{H}), 3.36-3.31(\mathrm{~m}, 1 \mathrm{H}, \mathrm{CH})$, (m, 1H, CH), 3.23-3.22 (m, 1H, CH), 3.20-3.18 (bs, 1H, OH), 2.98-2.78 (bs, 1H, OH), 2.04$1.88(\mathrm{~m}, 2 \mathrm{H}), 1.78-1.67(\mathrm{~m}, 2 \mathrm{H}), 1.28\left(\mathrm{~d}, J=6.4 \mathrm{~Hz}, 3 \mathrm{H}, \mathrm{CH}_{3}\right), 1.16(\mathrm{~d}, J=6.4 \mathrm{~Hz}, 3 \mathrm{H}$, $\left.\mathrm{CH}_{3}\right)$.

${ }^{13} \mathrm{C} \mathrm{NMR}\left(100 \mathrm{MHz}, \mathrm{CDCl}_{3}\right) \delta 162.5,156.8,138.5,136.9,128.7,128.5,128.3,128.2,127.9$, $127.7,99.6,92.8,79.3,78.2,75.4,71.8,71.0,69.2,67.4,67.0,66.8,61.0,55.6,38.2,29.8$, 17.2, 16.3. 
HR-ESI-MS (m/z): calcd. for $\mathrm{C}_{32} \mathrm{H}_{40} \mathrm{Cl}_{3} \mathrm{~N}_{5} \mathrm{O}_{10} \mathrm{Na}^{+},(\mathrm{M}+\mathrm{Na})^{+}$784.1710; found, 784.1714.

$\mathrm{N}$-benzyloxycarbonyl-3-aminopropanyl-O-4,6-O-benzylidene-3- $O$-benzyl-2- $O$-benzoyl-)$(1 \rightarrow 3 / 4)$ - $\beta$-D-glucopyranosyl (2-azido-2-deoxy-4/3-hydroxy)-(1 $\rightarrow 3)$ - $\alpha$-L-fucopyranosyl 2(trichloroacetyl carbonylamino)-2-deoxy-4- $O$-benzyl- $\beta$-D-fucopyranoside (3/26)

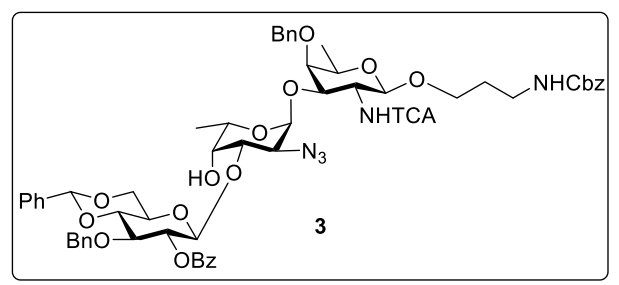

Thioglycoside donor 6 (0.266 g, $0.48 \mathrm{mmol})$ and disaccharide acceptor 5 (0.240 g, 0.27 mmol) were azeotroped with toluene and dissolved in anhydrous $\mathrm{CH}_{2} \mathrm{Cl}_{2}(10.7 \mathrm{~mL})$. Activated molecular sieves $(3 \AA, 0.70 \mathrm{~g})$ were added, and the mixture was stirred at room temperature for 30 minutes under nitrogen. NIS $(0.216 \mathrm{~g}, 0.96 \mathrm{mmol})$ was added then the mixture was cooled to $0{ }^{\circ} \mathrm{C}$ and TMSOTf $(17.4 \mu \mathrm{L}, 0.096 \mathrm{mmol})$ was added dropwise. After 30 mins, TLC (EtOAc:Hexane, 4:6, v/v) showed the acceptor has been converted into a less polar product. The mixture was neutralized with triethyl amine, filtered through a Celite bed using $\mathrm{CH}_{2} \mathrm{Cl}_{2}$. The organic layer was washed with aq. $\mathrm{Na}_{2} \mathrm{~S}_{2} \mathrm{O}_{3}$ solution, separated organic layer was dried over anhydrous $\mathrm{Na}_{2} \mathrm{SO}_{4}$, and concentrated in vacuo and purified by column chromatography on silica gel (3:7 ethyl acetate: pet ether) afforded trisaccharide $\mathbf{3}$ as white foam $(0.197 \mathrm{~g}, 67 \%)$ and the other trisaccharide $\mathbf{2 6}$ as a sticky foam (96 $\mathrm{mg}, 32 \%)$ : Compound 3.

$[\alpha]^{25} \mathrm{D}-3.38\left(c=0.2, \mathrm{CHCl}_{3}\right)$.

IR $\left(\mathrm{cm}^{-1}, \mathrm{CHCl}_{3}\right) v 3479,2933,2115,1717,1270,1101,790,752$.

${ }^{1} \mathrm{H}$ NMR (400 MHz, $\left.\mathrm{CDCl}_{3}\right) \delta$ 7.94-7.93 (m, 2H, ArH), 7.61-7.41 (m, 8H, ArH), 7.39-7.10 (m, 15H, ArH), 5.64 (s, 1H, PhCH), 5.26 (t, $J=8.2 \mathrm{~Hz}, 1 \mathrm{H}, \mathrm{H}-2 "), 5.13-5.07$ (m, 3H, NH, $\mathrm{CH}_{2}$ of Cbz), 4.93 (d, $\left.J=3.6 \mathrm{~Hz}, 1 \mathrm{H}, \mathrm{H}-1^{\prime}\right), 4.87-4.80$ (m, 3H, H-1, $\left.\mathrm{PhCH}\right), 4.73$ (d, $J=12.0$ $\mathrm{Hz}, 1 \mathrm{H}, \mathrm{PhCH}), 4.63-4.59$ (m, 2H, H-1"), 4.46-4.42 (m, 1H), 4.23-4.20 (m, 1H), 3.95-3.88 (m, 5H), 3.69-3.50 (m, 8H), 3.41-3.34 (m, 1H), 3.29-3.20 (m, 2H), 1.76-1.74 (m, 3H), 1.30 (d, $\left.J=6.4 \mathrm{~Hz}, 3 \mathrm{H}, \mathrm{CH}_{3}\right), 1.00\left(\mathrm{~d}, J=6.8 \mathrm{~Hz}, 3 \mathrm{H}, \mathrm{CH}_{3}\right)$.

${ }^{13} \mathrm{C} \mathrm{NMR}\left(100 \mathrm{MHz}, \mathrm{CDCl}_{3}\right) \delta 165.8,162.4,156.7,138.9,137.8,137.3,136.9,133.8,129.9$, $129.4,129.3,128.8,128.7,128.5,128.4,128.3,128.2$, 127.9, 127.7, 127.4, 126.2, 101.5, 
$101.2,100.0,99.6,92.7,81.5,80.3,78.0,77.8,76.9,75.7,74.3,74.2,71.0,69.5,68.8,67.5$, $66.7,66.3,58.4,55.8,38.3,29.8,17.2,16.1$.

HR-ESI-MS (m/z): calcd. for $\mathrm{C}_{59} \mathrm{H}_{64} \mathrm{Cl}_{3} \mathrm{~N}_{5} \mathrm{O}_{16} \mathrm{Na}^{+},(\mathrm{M}+\mathrm{Na})^{+}$1228.3295; found, 1228.3301 .

Compound 26:

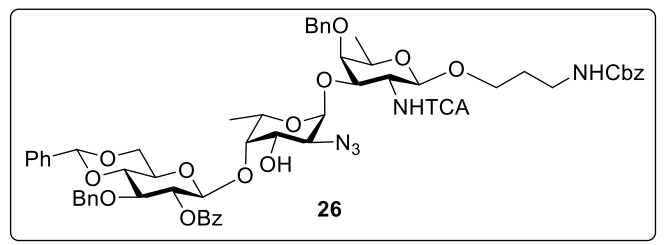

$[\alpha]^{25} \mathrm{D}-8.35\left(c=0.2, \mathrm{CHCl}_{3}\right)$.

IR $\left(\mathrm{cm}^{-1}, \mathrm{CHCl}_{3}\right) v 3646,2930,2115,1720,1264,1094,1070$.

${ }^{1} \mathrm{H}$ NMR $\left(500 \mathrm{MHz}, \mathrm{CDCl}_{3}\right) \delta$ 7.95-7.94 (m, 2H, ArH), 7.59-7.04 (m, 23H, ArH), $5.61(\mathrm{~s}$, $1 \mathrm{H}, \mathrm{CH}$ of benzylidine), 5.35 (t, $\left.J=8.5 \mathrm{~Hz}, 1 \mathrm{H}, \mathrm{H}-2^{\prime \prime}\right), 5.15-5.06\left(\mathrm{~m}, 4 \mathrm{H}, \mathrm{H}-1\right.$ ', $\mathrm{NH}, \mathrm{CH}_{2}$ of Cbz), 4.84-4.80 (m, 2H), 4.78-4.70 (m, 2H), 4.69-4.58 (m, 1H), 4.40 (dd, $J=10.2 \mathrm{~Hz}, 4.7$ $\mathrm{Hz}, 1 \mathrm{H},), 4.06-4.04(\mathrm{~m}, 1 \mathrm{H}), 3.95-3.76(\mathrm{~m}, 9 \mathrm{H}), 3.58-3.43(\mathrm{~m}, 7 \mathrm{H}), 3.33-3.31(\mathrm{~m}, 1 \mathrm{H}, \mathrm{CH})$, 3.24-3.21 (m, 1H, CH), 1.73-1.71 (m, 2H), $1.22\left(\mathrm{~d}, J=6.5 \mathrm{~Hz}, 3 \mathrm{H}, \mathrm{CH}_{3}\right), 0.82(\mathrm{~d}, J=6.5 \mathrm{~Hz}$, $\left.3 \mathrm{H}, \mathrm{CH}_{3}\right)$.

${ }^{13} \mathrm{C}$ NMR $\left(125 \mathrm{MHz}, \mathrm{CDCl}_{3}\right) \delta 165.0,162.5,156.7,138.5,137.6,137.0,133.6,130.1,129.7$, $129.4,128.7,128.6,128.57,128.52,128.4,128.2$, 127.9, 127.8, 127.7, 126.3, 126.2, 103.2, 101.6, 100.0, 99.4, 92.8, 83.4, 81.5, 78.8, 78.7, 75.2, 74.2, 73.4, 70.9, 68.5, 68.4, 67.4, 67.0, $66.9,66.6,61.8,55.3,38.3,29.7,17.2,16.1$

HR-ESI-MS (m/z): calcd. for $\mathrm{C}_{59} \mathrm{H}_{64} \mathrm{Cl}_{3} \mathrm{~N}_{5} \mathrm{O}_{16} \mathrm{Na}^{+}, 1228.3295 ;(\mathrm{M}+\mathrm{Na})^{+}$found, 1228.3298 .

$\mathrm{N}$-benzyloxycarbonyl-3-aminopropanyl-O-4,6-O-benzylidene-3- $O$-benzyl-2- $O$-benzoyl$(1 \rightarrow 3)$ - $\beta$-D-glucopyranosyl $\quad$ (4-acetyl-2-azido-2-deoxy)-(1 $\rightarrow 3)$ - $\alpha$-L-fucopyranosyl-2(trichloroacetyl carbonylamino)-2-deoxy-4- $O$-benzyl- $\beta$-D-fucopyranoside (27)

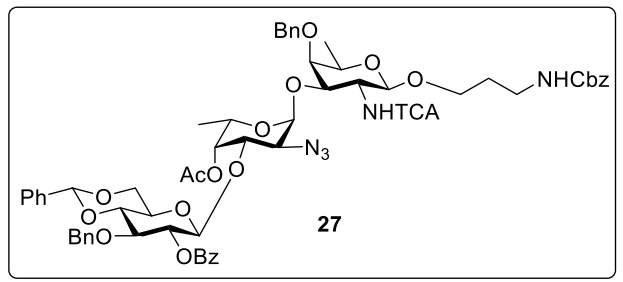


Compound 3 (45 mg, $0.04 \mathrm{mmol}$ ) was dissolved in $\mathrm{CH}_{2} \mathrm{Cl}_{2}(1 \mathrm{~mL})$ and pyridine $(0.01 \mathrm{~mL})$ and $\mathrm{AcCl}(0.01 \mathrm{~mL})$ were added slowly. After stirring the reaction mixture at room temperature for $1 \mathrm{~h}$, it was diluted with $\mathrm{CH}_{2} \mathrm{Cl}_{2}(1 \mathrm{~mL})$ and washed with brine, $1 \mathrm{~N} \mathrm{HCl}$ solution. Separated organic layer was dried over anhydrous $\mathrm{Na}_{2} \mathrm{SO}_{4}$, concentrated and purified by column chromatography on silica gel (25\% ethyl acetate: pet ether) to afford 27 as sticky solid (30 mg, 80\%).

$[\alpha]^{25} \mathrm{D}-56.50\left(c=0.02, \mathrm{CHCl}_{3}\right)$.

IR $\left(\mathrm{cm}^{-1}, \mathrm{CHCl}_{3}\right) v 3368,2114,1715,1269,1092,758$.

${ }^{1} \mathrm{H}$ NMR (400 MHz, $\left.\mathrm{CDCl}_{3}\right) \delta 8.02-8.00(\mathrm{~m}, 2 \mathrm{H}, \mathrm{ArH}), 7.59-7.04(\mathrm{~m}, 23 \mathrm{H}, \mathrm{ArH}), 5.62(\mathrm{~s}$, 1H, CH of benzylidine), 5.27 ( $\mathrm{t}, J=8.0 \mathrm{~Hz}, 1 \mathrm{H}, \mathrm{H}-2 "), 5.10-5.07$ (m, 3H, NH, $\mathrm{CH}_{2}$ of Cbz), 4.96-4.89 (m, 3H), 4.84-4.64 (m, 5H, CHHPh), 4.32-4.29 (m, 2H), 4.14-4.12 (m, 1H), 3.94$3.83(\mathrm{~m}, 5 \mathrm{H}), 3.77-3.65(\mathrm{~m}, 3 \mathrm{H}), 3.53-3.44(\mathrm{~m}, 3 \mathrm{H}), 3.29-3.26(\mathrm{~m}, 3 \mathrm{H}), 1.71-1.69(\mathrm{~m}, 2 \mathrm{H})$, $1.41\left(\mathrm{~s}, 3 \mathrm{H}, \mathrm{COCH}_{3}\right), 1.33\left(\mathrm{~d}, J=6.0 \mathrm{~Hz}, 3 \mathrm{H}, \mathrm{CH}_{3}\right), 0.91\left(\mathrm{~d}, J=6.0 \mathrm{~Hz}, 3 \mathrm{H}, \mathrm{CH}_{3}\right)$.

${ }^{13} \mathrm{C}$ NMR $\left(100 \mathrm{MHz}, \mathrm{CDCl}_{3}\right) \delta 170.3,164.9,162.5,156.7,138.7,137.9,137.4,136.9,134.6$, $133.5,133.4,130.4,130.3,130.1,129.9$, 129.8, 129.3, 129.2, 128.8, 128.7, 128.6, 128.5, 128.4, 128.3, 128.2, 128.1, 128.0, 127.8, 127.6, 127.5, 126.2, 101.5, 100.1, 99.1, 97.8, 92.6, $81.7,79.9,75.5,74.9,74.2,73.5,73.4,71.3,71.1,70.4,68.9,68.5,67.7,66.7,66.6,65.7$, $62.2,58.2,58.1,56.3,38.4,29.9,20.0,17.3,16.3$.

HR-ESI-MS (m/z): calcd. for $\mathrm{C}_{61} \mathrm{H}_{66} \mathrm{Cl}_{3} \mathrm{~N}_{5} \mathrm{O}_{17},(\mathrm{M}+\mathrm{Na})^{+}$1270.3402; found, 1270.3403 .

\section{$N$-benzyloxycarbonyl-3-aminopropanyl-O-4,6-O-benzylidene-3- $O$-benzyl-2- $O$-hydroxy-} )-(1 $\rightarrow 3)$ - $\beta$-D-glucopyranosyl $\quad(2$-azido-2-deoxy-4- $O$-hydroxy $)-(1 \rightarrow 3)-\alpha$-L-fucopyranosyl2-(trichloroacetyl carbonylamino)-2-deoxy-4- $O$-benzyl- $\beta$-D-fucopyranoside (28)

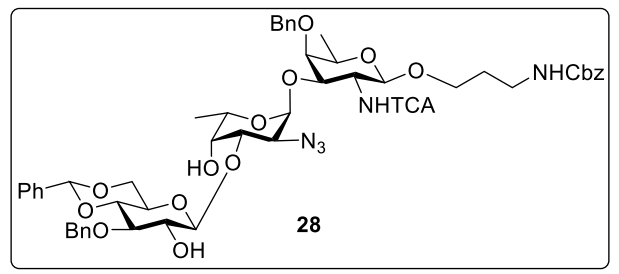

$\mathrm{NaOMe}(30 \mathrm{mg})$ was added to a stirred solution of compound 3 (66 mg, $0.054 \mathrm{mmol})$ in $\mathrm{MeOH}(1 \mathrm{~mL})$. After 30 minutes reaction mixture was neutralized with Amberlite IR 120 $\left(\mathrm{H}^{+}, 0.13 \mathrm{~g}\right)$ resin. The reaction mixture was filtered and concentrated under reduced pressure 
and purified by column chromatography on silica gel (4:6 ethyl acetate: pet ether) to afford desired compound $\mathbf{2 8}$ as viscous liquid ( $57 \mathrm{mg}, \mathbf{9 8 \%}$ ).

$[\alpha]^{25} \mathrm{D}-9.53\left(c=0.25, \mathrm{CHCl}_{3}\right)$.

IR $\left(\mathrm{cm}^{-1}, \mathrm{CHCl}_{3}\right) v 3369,2328,1662,791,715$.

${ }^{1} \mathrm{H}$ NMR $\left(500 \mathrm{MHz}, \mathrm{CDCl}_{3}\right) \delta$ 7.51-7.26 (m, 20H, ArH), 5.59 (s, 1H, CH of benzylidene), 5.07-4.94 (m, 4H, NH, $\mathrm{CH}_{2}$ of Cbz), 4.89-4.86 (m, 4H), 4.43-4.39 (m, 2H), 3.99-3.93 (m, $3 \mathrm{H}), 3.88-3.84(\mathrm{~m}, 2 \mathrm{H}), 3.78-3.71(\mathrm{~m}, 3 \mathrm{H}), 3.63-3.53(\mathrm{~m}, 8 \mathrm{H}), 3.46-3.43(\mathrm{~m}, 2 \mathrm{H}), 3.34-3.33$ (m, 1H), 3.25-3.23 (m, 2H), 1.75-1.74 (m, 3H), 1.29 (d, $\left.J=6.0 \mathrm{~Hz}, 3 \mathrm{H}, \mathrm{CH}_{3}\right), 1.17(\mathrm{~d}, J=6.0$ $\left.\mathrm{Hz}, 3 \mathrm{H}, \mathrm{CH}_{3}\right)$.

${ }^{13} \mathrm{C} \mathrm{NMR}\left(125 \mathrm{MHz}, \mathrm{CDCl}_{3}\right) \delta 162.5,156.7,138.9,138.2,137.3,136.9,129.3,128.8,128.6$, $128.5,128.4,128.3,128.26,128.21,127.9,127.7,127.4$, 126.1, 101.4, 99.9, 99.6, 92.7, 81.4, 80.6, 76.3, 75.6, 74.7, 73.6, 71.0, 68.8, 68.2, 67.5, 67.0, 66.7, 66.4, 60.9, 58.5, 55.4, 38.3, $29.7,17.2,16.4$.

HR-ESI-MS (m/z): calcd. for $\mathrm{C}_{52} \mathrm{H}_{60} \mathrm{Cl}_{3} \mathrm{~N}_{5} \mathrm{O}_{15} \mathrm{Na}^{+},(\mathrm{M}+\mathrm{Na})^{+} 1124.3030$; found, 1124.3056 .

$\mathrm{N}$-benzyloxycarbonyl-3-aminopropanyl-O-4,6-O-benzylidene-3- $O$-benzyl-2- $O$-hydroxy)$(1 \rightarrow 3)$ - $\beta$-D-glucopyranosyl (2-acetamido-2-deoxy-4- $O$-hydroxy)-(1 $\rightarrow 3)-\alpha$-Lfucopyranosyl -2-(acetamido)-2-deoxy-4- $O$-benzyl- $\beta$-D-fucopyranoside (29)

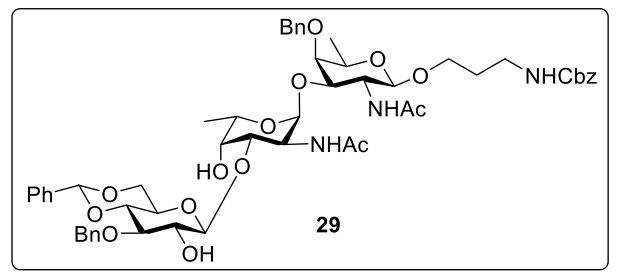

Activated zinc dust $(0.100 \mathrm{~g})$ and $\mathrm{AcOH}(0.05 \mathrm{~mL})$ were added in compound 28 (38 $\mathrm{mg}$, $0.034 \mathrm{mmol})$ in THF $(1 \mathrm{~mL})$ and stirred at room temperature for $12 \mathrm{~h}$. Amine compound was filtered through celite bed using EtOAc as a solvent, concentrated under reduced pressure and dried under high vacuum for $1 \mathrm{~h}$. Then dried amine compound was dissolved in $\mathrm{MeOH}(1$ $\mathrm{mL}), \mathrm{CH}_{2} \mathrm{Cl}_{2}(0.1 \mathrm{~mL}), \mathrm{Et}_{3} \mathrm{~N}(0.2 \mathrm{~mL})$ and $\mathrm{Ac}_{2} \mathrm{O}(0.05 \mathrm{~mL})$ were added and stirred it at for $3 \mathrm{~h}$ and concentrated under reduced pressure and purified by column chromatography on silica gel (1:9 methanol: ethyl acetate) to obtain NHAc compound 29 as a colourless foam (26 $\mathrm{mg}, 78 \%)$. 
$[\alpha]^{25} \mathrm{D}+21.40\left(c=0.15, \mathrm{CHCl}_{3}\right)$.

IR $\left(\mathrm{cm}^{-1}, \mathrm{CHCl}_{3}\right) v 3421,2929,1649,1258,1072,755$.

${ }^{1} \mathrm{H}$ NMR (400 MHz, $\left.\mathrm{CDCl}_{3}\right) \delta$ 7.38-7.71 (m, 20H, ArH), $5.23(\mathrm{~s}, 1 \mathrm{H}, \mathrm{CH}$ of benzylidine), 5.13-5.05 (m, 4H, $\mathrm{CH}_{2}$ of Cbz), 4.88-4.80 (m, 4H), 4.72 (d, J=12.0 Hz, 1H, CHPh), 4.40$4.39(\mathrm{~m}, 2 \mathrm{H}), 4.13-4.01(\mathrm{~m}, 3 \mathrm{H}), 3.94-3.92(\mathrm{~m}, 1 \mathrm{H}), 3.80-3.66(\mathrm{~m}, 3 \mathrm{H}), 3.58-3.51(\mathrm{~m}, 4 \mathrm{H})$, 3.43-3.30 (m, 5H), 3.20-3.17 (m, 2H), 3.14-3.07 (m, 3H), 2.17 (s, 3H, NHAc), 1.94 (s, 3H, NHAc), $1.77-1.67(\mathrm{~m}, 1 \mathrm{H}), 1.59-1.56(\mathrm{~m}, 1 \mathrm{H}), 1.36\left(\mathrm{~d}, J=6.8 \mathrm{~Hz}, 3 \mathrm{H}, \mathrm{CH}_{3}\right), 1.33(\mathrm{~d}, J=6.0$ $\left.\mathrm{Hz}, 3 \mathrm{H}, \mathrm{CH}_{3}\right)$.

${ }^{13} \mathrm{C} \mathrm{NMR}\left(125 \mathrm{MHz}, \mathrm{CDCl}_{3}\right) \delta 173.3,172.4,157.2,139.0,138.8,137.8,137.0,128.7,128.5$, $128.47,128.40,128.3,128.2,127.9,127.2$, 126.2, 104.8, 101.8, 100.8, 100.7, 81.5, 80.8, $80.3,75.6,75.4,73.7,71.0,69.7,68.3,67.2,67.0,66.5,65.9,51.9,47.4,46.2,36.9,29.9$, $23.5,23.3,17.3,16.7$.

HR-ESI-MS (m/z): calcd. for $\mathrm{C}_{54} \mathrm{H}_{67} \mathrm{~N}_{3} \mathrm{O}_{16} \mathrm{Na}^{+},(\mathrm{M}+\mathrm{Na})^{+}$1036.4414; found, 1036.4417.

3-amino-propanyl-( $1 \rightarrow 3)$ - $\beta$-D-glucopyranosyl-2-acetamido-2-deoxy-( $\rightarrow 3)$ - $\alpha$-Lfucopyranosyl-2-(acetamido)-2-deoxy- $\beta$-D-fucopyranoside (1)

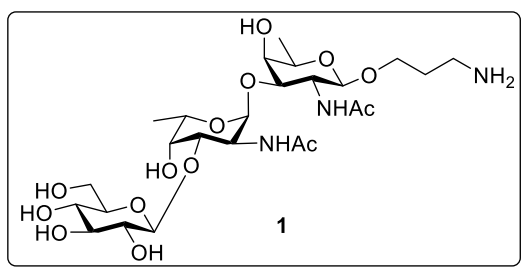

The compound 29 (0.026 g, $0.026 \mathrm{mmol})$ was dissolved in $\mathrm{EtOH}(2.6 \mathrm{~mL})$. To this solution $\mathrm{Pd}(\mathrm{OH})_{2} / \mathrm{C}(0.08 \mathrm{~g}, 20 \% \mathrm{Pd}$ content $)$ was added. The reaction was stirred under hydrogen atmosphere for $8 \mathrm{~h}$. The reaction mixture was filtered through a Celite bed with methanol as eluent. Filtrate was concentrated under reduced pressure and dried under high vacuum to obtain 1 as a sticky solid (14 mg, 92\%).

$[\alpha]^{25} \mathrm{D}+34.67\left(c=0.15, \mathrm{CHCl}_{3}\right)$.

IR $\left(\mathrm{cm}^{-1}, \mathrm{CHCl}_{3}\right) v 3407,2505,1659,1452,1216,760$.

${ }^{1} \mathrm{H}$ NMR (500 MHz, D $\left.2 \mathrm{O}\right) \delta 4.95(\mathrm{bs}, 1 \mathrm{H}), 4.44(\mathrm{~d}, J=8.0 \mathrm{~Hz}, 1 \mathrm{H}), 4.40-4.29$ (m, 2H), 4.12 (bs, 2H), 4.02-4.01 (m, 2H), 3.95 (s, 1H), 3.89-3.84 (m, 6H), 3.70-3.57 (m, 8H), 3.41-3.33 
(m, 4H), 3.31-3.26 (m, 2H), 3.21-3.18 (m, 2H), 3.00-2.96 (m, 2H), 1.95 (s, 3H, NHAc), 1.92 (s, 3H, NHAc), 1.19 (d, $\left.J=6.0 \mathrm{~Hz}, 3 \mathrm{H}, \mathrm{CH}_{3}\right), 1.16\left(\mathrm{~d}, J=6.0 \mathrm{~Hz}, 3 \mathrm{H}, \mathrm{CH}_{3}\right)$.

${ }^{13} \mathrm{C}$ NMR (125 MHz, $\left.\mathrm{D}_{2} \mathrm{O}\right) \delta 174.34,174.30,101.4,100.3,99.1,77.0,76.1,75.6,74.9,72.9$, 70.7, 69.7, 68.5, 67.9, 66.1, 60.9, 55.6, 51.3, 47.9, 42.2, 37.7, 22.3, 20.2, 15.5, 15.4 .

HR-ESI-MS (m/z): calcd. for $\mathrm{C}_{25} \mathrm{H}_{45} \mathrm{~N}_{3} \mathrm{O}_{14} \mathrm{H}^{+},(\mathrm{M}+\mathrm{H})^{+}$612.2974; found, 612.2969.

$\mathrm{N}$-benzyloxycarbonyl-3-aminopropanyl-O-(2-azido-2-deoxy-4- $O$-hydroxy-3-Onaphthylmethyl- $\alpha$-L-fucopyranosyl)-(1 $\rightarrow 3$ )-2-(trichloroacetyl carbonylamino)-2-deoxy4- $O$-benzyl- $\beta$-D-fucopyranoside (30c):

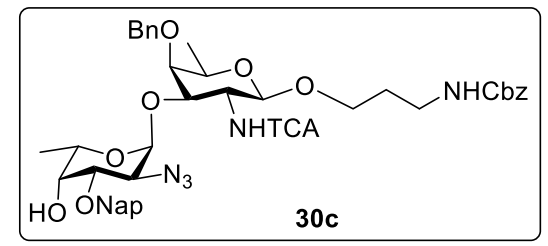

Disaccharide diol Compound 5 (0.230 g, $0.302 \mathrm{mmol})$ was dissolved in dry toluene $(4.5 \mathrm{~mL})$, to this stirred solution $\mathrm{Bu}_{2} \mathrm{SnO}(0.115 \mathrm{~g}, 0.45 \mathrm{mmol})$ was added and reaction mixture was kept at $110^{\circ} \mathrm{C}$ for $3 \mathrm{~h}$. After that reaction mixture was cooled to $\mathrm{rt}$. In that TBAB $(97 \mathrm{mg}$, $0.44 \mathrm{mmol})$ followed by Naphthylmethyl bromide $(0.280 \mathrm{~g}, 0.87 \mathrm{mmol})$ were added and reaction mixture was kept for stirring at $60{ }^{\circ} \mathrm{C}$ temperature under reflux condition. After $1 \mathrm{~h}$, reaction was completed (confirmed by TLC) which was concentrated and purified by column chromatography on silica gel (3:7 ethyl acetate: pet ether) to afford 30c as off-white foam $(0.215 \mathrm{~g}, 79 \%)$.

$[\alpha]^{25} \mathrm{D}-58.116\left(c=0.10, \mathrm{CHCl}_{3}\right)$.

IR $\left(\mathrm{cm}^{-1}, \mathrm{CHCl}_{3}\right) v 3836,3746,2950,2317,2113,1720,1532,1374,1256,1101,756,615$.

${ }^{1} \mathrm{H}$ NMR $\left(400 \mathrm{MHz}, \mathrm{CDCl}_{3}\right) \delta$ 7.85-7.77 (m, 4H, ArH), 7.51-7.47 (m, 3H, ArH), 7.36-7.24 (m, 10H, ArH), 5.19-5.08 (m, 3H, $\mathrm{CH}_{2} \mathrm{Cbz}, \mathrm{NH}$ ), 4.97 (bs, 1H, H-1'), 4.80-4.69 (m, 5H, H-1, $\mathrm{CH}_{2}$ of Bn \& Nap), 4.17-4.14 (m, 1H), 4.01-3.76 (m, 5H), 3.65-3.35 (m, 6H), 3.25-3.20 (m, 1H), 1.78-1.72 (m, 2H, CH2 Linker) 1.27 (d, $\left.J=6.52 \mathrm{~Hz}, 3 \mathrm{H}, \mathrm{CH}_{3}\right), 1.19$ (d, $J=6.56 \mathrm{~Hz}, 3 \mathrm{H}$, $\left.\mathrm{CH}_{3}\right)$.

${ }^{13} \mathrm{C} \mathrm{NMR}\left(125 \mathrm{MHz}, \mathrm{CDCl}_{3}\right) \delta 162.2,156.6,138.5,136.8,134.5,133.2,133.1,128.6,128.5$, 128.4, 128.01, 127.98, 127.8, 127.6, 127.4, 126.8, 126.4, 126.3, 125.6, 99.7, 99.4, 92.7, 79.2, $78.4,77.02,75.3,71.9,70.8,68.5,67.2,66.5,59.5,55.4,38.05,29.6,17.08,16.25$. 
HR-ESI-MS (m/z): calcd. for $\mathrm{C}_{43} \mathrm{H}_{48} \mathrm{Cl}_{3} \mathrm{~N}_{5} \mathrm{O}_{10} \mathrm{Na}^{+},(\mathrm{M}+\mathrm{Na})^{+}$924.2340; found, 924.2340.

$\mathrm{N}$-benzyloxycarbonyl-3-aminopropanyl-O-4,6-O-benzylidene-3- $\mathrm{O}$-benzyl-2-O-benzoyl-)$(1 \rightarrow 4)$ - $\beta$-D-glucopyranosyl $\quad$ (2-azido-2-deoxy-3- $O$-naphthylmethyl)-(1 $\rightarrow 3)$ - $\alpha$-Lfucopyranosyl 2-(trichloroacetyl carbonylamino)-2-deoxy-4- $O$-benzyl- $\beta$-Dfucopyranoside (4c):

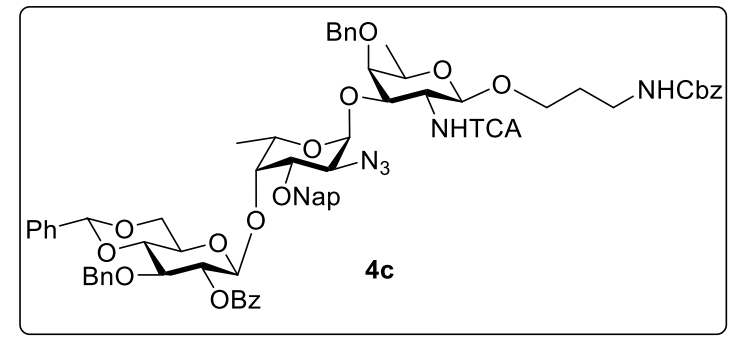

Thioglycoside donor 6 (0.266 g, $0.48 \mathrm{mmol})$ and disaccharide acceptor 30c $(0.240 \mathrm{~g}, 0.27$ mmol) were azeotroped with toluene and dissolved in anhydrous $\mathrm{CH}_{2} \mathrm{Cl}_{2}(10.7 \mathrm{~mL})$. Activated molecular sieves $(3 \AA, 0.70 \mathrm{~g})$ were added, and the mixture was stirred at room temperature for 30 minutes under nitrogen. NIS $(0.216 \mathrm{~g}, 0.96 \mathrm{mmol})$ was added then the mixture was cooled to $-60{ }^{\circ} \mathrm{C}$ and TMSOTf $(17.4 \mu \mathrm{L}, 0.096 \mathrm{mmol})$ were added drop wise. After 10 minutes, reaction was allowed to warmed to $-10{ }^{\circ} \mathrm{C}$ and left for $2-3 \mathrm{~h}$. TLC (EtOAc:Pet ether, 4:6, v/v) showed the acceptor has been converted into a less polar product. The mixture was neutralized with triethyl amine, filtered through Celite bed and diluted with $\mathrm{CH}_{2} \mathrm{Cl}_{2}$. The organic layer was washed with aq. $\mathrm{Na}_{2} \mathrm{~S}_{2} \mathrm{O}_{3}$ solution, dried over anhydrous $\mathrm{Na}_{2} \mathrm{SO}_{4}$, and concentrated in vacuo. Purified by column chromatography on silica gel (4:6 ethyl acetate: pet ether) to afford trisaccharide $\mathbf{4 c}$ as white foam $(0.300 \mathrm{~g}, 84 \%)$.

$[\alpha]_{\mathrm{D}}^{25}=-30.15\left(c=0.50, \mathrm{CHCl}_{3}\right)$.

IR $\left(\mathrm{cm}^{-1}, \mathrm{CHCl}_{3}\right) \vee 3341,2936,2113,1715,1525,1453,1372,1266,1178,1097,1070$, 1028, 999, 819, 698 .

${ }^{1} \mathrm{H}$ NMR (400 MHz, $\left.\mathrm{CDCl}_{3}\right) \delta$ 7.94-7.83 (m, 6H, ArH), 7.60-7.07 (m, 26H, ArH), 5.41 (s, $1 \mathrm{H}, \mathrm{CH}$ of benzylidene), 5.38 (t, $J=8.30 \mathrm{~Hz}, 1 \mathrm{H}, \mathrm{H}-2 "), 5.20$ (bs, 1H, NH), 5.07 (bs, 2H, $\mathrm{CH}_{2}$ of Cbz), 4.88-4.63 (m, 9H), 4.18-3.73 (m, 10H), 3.58-3.19 (m, 7H), 1.74-1.71 (m, 2H, $\mathrm{CH}_{2}$ linker), 1.22 (d, $\left.J=6.21 \mathrm{~Hz}, 3 \mathrm{H}, \mathrm{CH}_{3}\right), 0.96\left(\mathrm{~d}, J=6.50 \mathrm{~Hz}, 3 \mathrm{H}, \mathrm{CH}_{3}\right)$.

${ }^{13} \mathrm{C} \mathrm{NMR}\left(100 \mathrm{MHz}, \mathrm{CDCl}_{3}\right) \delta 164.9,162.2,156.6,138.4,137.8,137.2,136.9,135.2,133.3$, 133.2, 133.04, 129.8, 129.04, 128.5, 128.4, 128.3, 128.2, 128.1, 128.0, 127.9, 127.8, 127.6, 
$126.55,126.50,126.4,126.1,125.3,101.97,101.2,100.02,99.2,92.7,81.4,78.9,78.6,77.9$, 76.9, 76.2, 75.0, 73.8, 73.7, 71.7, 70.7, 68.4, 67.2, 67.1, 66.5, 66.4, 59.8, 54.98, 38.1, 29.7, 17.0, 16.4 .

HR-ESI-MS (m/z): calcd. for $\mathrm{C}_{70} \mathrm{H}_{72} \mathrm{Cl}_{3} \mathrm{~N}_{5} \mathrm{NaO}_{16} \mathrm{Na}^{+},(\mathrm{M}+\mathrm{Na})^{+}$1368.3926; found, 1368.3925 .

\section{$\mathrm{N}$-benzyloxycarbonyl-3-aminopropanyl-O-4,6-O-benzylidene-3-O-benzyl-2-O-}

hydroxy- )-(1 $\rightarrow 4)$ - $\beta$-D-glucopyranosyl (2-azido-2-deoxy-3- $O$-naphthylmethyl)-( $1 \rightarrow 3)$ - $\alpha$ -

L-fucopyranosyl

2-(trichloroacetylcarbonylamino)-2-deoxy-4-O-benzyl- $\beta$-D-

fucopyranoside (31):

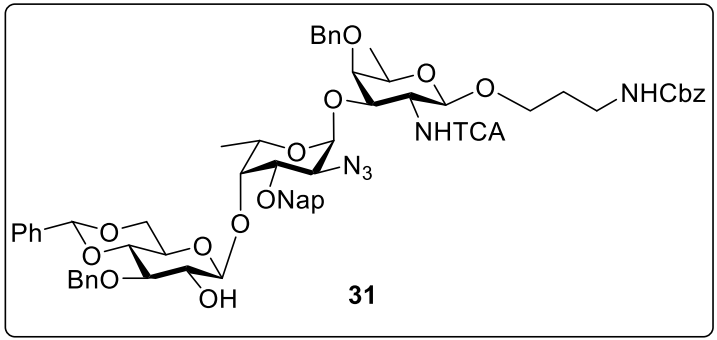

$\mathrm{NaOMe}(0.114 \mathrm{~g})$ was added to a stirred solution of compound $4 \mathrm{c}(0.192 \mathrm{~g}, 0.143 \mathrm{mmol})$ in $\mathrm{MeOH}$ :DCM (4:2 mL). After $2 \mathrm{~h}$ reaction mixture was concentrated under reduced pressure and purified by column chromatography on silica gel (4:6 ethyl acetate: pet ether) to afford trisaccharide $\mathbf{3 1}$ as white foam $(0.160 \mathrm{~g}, 90 \%)$.

$[\alpha]^{25} \mathrm{D}-41.354\left(c=0.20, \mathrm{CHCl}_{3}\right)$.

IR $\left(\mathrm{cm}^{-1}, \mathrm{CHCl}_{3}\right) v 3870,3819,3749,2938,2113,1726,1524,1472,1262,1100,755,677$.

${ }^{1} \mathrm{H}$ NMR (500 MHz, $\left.\mathrm{CDCl}_{3}\right) \delta$ 7.87-7.79 (m, 4H, ArH), 7.39-7.15 (m, 23H, ArH), 5.34 (s, 1H, CH of benzylidine), 5.09-5.07 (m, 1H, NH of Cbz), 4.98 (s, 2H, $\mathrm{CH}_{2}$ of Cbz), 4.92 (d, J $\left.=3.64 \mathrm{~Hz}, 1 \mathrm{H}, \mathrm{H}-1^{\prime}\right)$, 4.81-4.40 (m, 8H), 4.18-3.77 (m, 7H), 3.68-3.14 (m, 12H), 1.67-1.64 (m, 2H, $\mathrm{CH}_{2}$ linker), 1.20 (d, $\left.J=6.18 \mathrm{~Hz}, 3 \mathrm{H}, \mathrm{CH}_{3}\right), 1.16\left(\mathrm{~d}, J=6.59 \mathrm{~Hz}, 3 \mathrm{H}, \mathrm{CH}_{3}\right)$.

${ }^{13} \mathrm{C} \mathrm{NMR}\left(125 \mathrm{MHz}, \mathrm{CDCl}_{3}\right) \delta 162.3,156.6,138.6,138.5,137.3,136.9,134.8,133.4,133.2$, $129.1,128.6,128.5,128.4,128.3,128.1,128.08,127.9$, 127.8, 127.7, 127.4, 126.5, 126.4, 126.2, 126.1, 125.6, 103.9, 101.3, 99.7, 99.5, 92.8, 81.3, 80.8, 79.6, 78.5, 75.8, 75.4, 74.8, $74.1,71.6,70.9,68.5,67.5,67.3,66.7,66.6,59.7,55.6,38.2,29.7,17.2,16.8$.

HR-ESI-MS (m/z): calcd. for $\mathrm{C}_{63} \mathrm{H}_{68} \mathrm{Cl}_{3} \mathrm{~N}_{5} \mathrm{O}_{15} \mathrm{Na}^{+},(\mathrm{M}+\mathrm{Na})^{+}$1264.3660; found, 1264.3661 . 


\section{$\mathrm{N}$-benzyloxycarbonyl-3-aminopropanyl-O-4,6- $\mathrm{O}$-benzylidene-3- $\mathrm{O}$-benzyl-2-azido-2-}

deoxy-)-(1 $\rightarrow 4)$ - $\beta$-D-mannopyranosyl (2-azido-2-deoxy-3- $O$-naphthylmethyl)-( $\rightarrow 3$ )- $\alpha$-Lfucopyranosyl 2-(trichloroacetylcarbonylamino)-2-deoxy-4-O-benzyl- $\beta$-Dfucopyranoside (32):

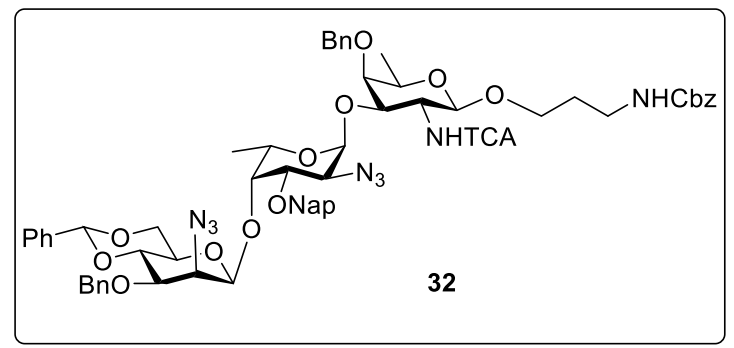

Trifluoromethanesulfonic anhydride $(0.05 \mathrm{~mL}, 0.27 \mathrm{mmol})$ was added to a solution of compound $31(0.222 \mathrm{~g}, 0.18 \mathrm{mmol})$ in pyridine $(0.044 \mathrm{~mL}, 0.54 \mathrm{mmol})$ and $\mathrm{CH}_{2} \mathrm{Cl}_{2}(6.3 \mathrm{~mL})$ at $0{ }^{\circ} \mathrm{C}$. After 30 mins of stirring, the reaction was quenched by addition of ice water and the mixture was extracted with $\mathrm{CH}_{2} \mathrm{Cl}_{2}(2 \times 5 \mathrm{~mL})$. The combined organic layer was washed with aq. $\mathrm{NaHCO}_{3}$ solution, dried over anhydrous $\mathrm{Na}_{2} \mathrm{SO}_{4}$, filtered and concentrated which was used for the next step without any purification. To a solution of the triflate in Toluene $(7 \mathrm{~mL})$ was added tetrabutylammonium azide $(0.154 \mathrm{~g}, 0.54 \mathrm{mmol})$ at $60{ }^{\circ} \mathrm{C}$. After stirring at the same temperature for $3 \mathrm{~h}$, the residue was purified by column chromatography on silica gel (4:6 ethyl acetate: pet ether) to afford compound $\mathbf{3 2}$ as viscous liquid $(0.160 \mathrm{~g}, 71 \%$ over 2 steps).

$[\alpha]^{25} \mathrm{D}-53.716\left(c=0.3, \mathrm{CHCl}_{3}\right)$.

IR $\left(\mathrm{cm}^{-1}, \mathrm{CHCl}_{3}\right) v 3747,3737,2888,2112,1714,1525,1374,1361,1252,1178,1098,821$, $753,699$.

${ }^{1} \mathrm{H}$ NMR (500 MHz, $\left.\mathrm{CDCl}_{3}\right) \delta$ 7.88-7.86 (m, 4H, ArH), 7.55-7.29 (m, 23H, ArH), 5.37 (s, 1H, $\mathrm{CH}$ of benzylidine), 5.21 (m, 1H, $\mathrm{NH}$ of $\mathrm{Cbz}$ ), 5.16-5.11 (bs, 2H, $\mathrm{CH}_{2}$ of $\mathrm{Cbz}$ ), 5.01 (bs, 1H, H1'), 4.90-4.65 (m, 8H), 4.16-3.83 (m, 10H), 3.65-3.17 (m, 8H), 1.79 (m, 2H linker), $1.30\left(\mathrm{~d}, J=5.05 \mathrm{~Hz}, 3 \mathrm{H}, \mathrm{CH}_{3}\right), 1.18\left(\mathrm{~d}, J=6.07 \mathrm{~Hz}, 3 \mathrm{H}, \mathrm{CH}_{3}\right)$.

${ }^{13} \mathrm{C} \mathrm{NMR}\left(125 \mathrm{MHz}, \mathrm{CDCl}_{3}\right) \delta 162.3,156.6,138.4,137.8,137.2,136.9,135.3,133.3,132.9$, 129.0, 128.5, 128.4, 128.3, 128.2, 128.1, 127.99, 127.95, 127.8, 127.7, 127.6, 127.5, 126.2, 126.0, 125.9, 125.2, 101.5, 101.08, 99.05, 92.7, 81.4, 78.9, 78.6, 78.2, 77.3, 77.1, 76.8, 76.0, 75.9, 75.4, 75.2, 72.9, 70.9, 70.8, 68.0, 67.5, 67.2, 67.1, 66.5, 63.2, 59.6, 55.1, 38.1, 29.6, 17.04, 17.01. 
HR-ESI-MS (m/z): calcd. for $\mathrm{C}_{63} \mathrm{H}_{67} \mathrm{C}_{13} \mathrm{~N}_{8} \mathrm{O}_{14} \mathrm{Na}^{+},(\mathrm{M}+\mathrm{Na})^{+}$1289.3726; found, 1289.3725.

\section{$\mathrm{N}$-benzyloxycarbonyl-3-aminopropanyl-O-4,6-O-benzylidene-3- $O$-benzyl-2-azido-2-}

deoxy-)-(1 $\rightarrow 4)$ - $\beta$-D-mannopyranosyl (2-azido-2-deoxy-3- $O$-acetyl)-( $1 \rightarrow 3)-\alpha$-L-

fucopyranosyl 2-(trichloroacetylcarbonylamino)-2-deoxy-4- $O$-benzyl- $\beta$-D-

fucopyranoside (33):

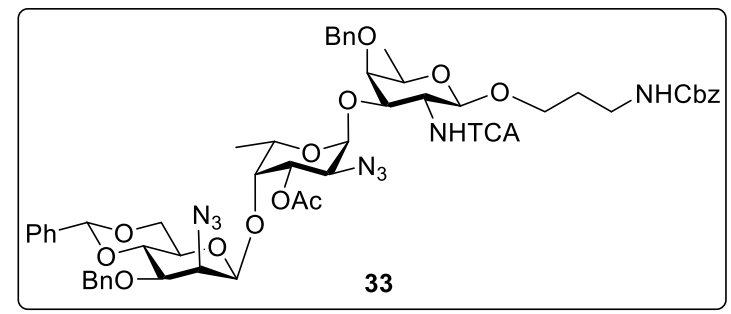

To a solution of trisaccharide $32(86 \mathrm{mg}, 0.07 \mathrm{mmol})$ in $\mathrm{CH}_{2} \mathrm{Cl}_{2}(1.2 \mathrm{~mL}), \mathrm{H}_{2} \mathrm{O}(0.5 \mathrm{~mL})$ was added, followed by the addition of DDQ $(24 \mathrm{mg}, 0.102 \mathrm{mmol})$. The reaction mixture was vigorously stirred at rt for 45 mins, after which TLC (EtOAc:Hexane, 4:6, v/v) showed the reaction had gone to completion. $\mathrm{R}_{f}=0.4$ (EtOAc:Pet ether). The mixture was diluted with $\mathrm{CH}_{2} \mathrm{Cl}_{2}(2 \times 10 \mathrm{~mL})$ and washed with sat. $\mathrm{NaHCO}_{3}(20 \mathrm{~mL})$. The organic layer was dried over anhydrous $\mathrm{Na}_{2} \mathrm{SO}_{4}$, filtered and the filtrate was concentrated in vacuo to afford the intermediate alcohol, which was dissolved in pyridine: $\mathrm{Ac}_{2} \mathrm{O}(2: 1,1.5 \mathrm{~mL})$. To the resulting solution, DMAP was added (5 mg, cat.) and stirring was continued at $\mathrm{rt}$ for $4 \mathrm{~h}$, after which the starting material had been fully consumed. The reaction mixture was concentrated in vacuo and the residue was purified by column chromatography on silica gel (3:7 ethyl acetate: pet ether) to afford the compound $\mathbf{3 3}$ as a white foam $0.73 \mathrm{~g}$ (92\%, over two steps).

$[\alpha]^{25} \mathrm{D}-23.035\left(c=0.1, \mathrm{CHCl}_{3}\right)$.

IR $\left(\mathrm{cm}^{-1}, \mathrm{CHCl}_{3}\right) v 3819,3747,2920,2113,1740,1375,1262,1100,764,621$.

${ }^{1} \mathrm{H}$ NMR (500 MHz, $\left.\mathrm{CDCl}_{3}\right) \delta$ 7.47-7.45 (m, 2H, ArH), 7.39-7.28 (m, 18H, ArH), 5.57 (s, $1 \mathrm{H}, \mathrm{CH}$ of benzylidine), $5.16(\mathrm{~m}, 1 \mathrm{H}, \mathrm{NH}), 5.07$ (s, 2H, $\mathrm{CH}_{2}$ of $\left.\mathrm{Cbz}\right), 4.99-4.96(\mathrm{~m}, 2 \mathrm{H})$, 4.87-4.72 (m, 5H), 4.48 (s, 1H), 4.28-3.91 (m, 9H), 3.79 (t, J = 10.24, 1H, H-2), 3.67-3.22 (m, 7H), 2.12 (s, 3H, $\left.\mathrm{CH}_{3}\right), 1.75-1.69$ (m, 2H linker), 1.28 (d, J=6.29 Hz, 3H, $\left.\mathrm{CH}_{3}\right), 1.07$ (d, $\left.J=6.50 \mathrm{~Hz}, 3 \mathrm{H}, \mathrm{CH}_{3}\right)$.

${ }^{13} \mathrm{C}$ NMR $\left(125 \mathrm{MHz}, \mathrm{CDCl}_{3}\right) \delta 170.2,162.2,156.5,138.1,137.6,137.0,129.1,128.5$, $128.48,128.45,128.3,127.99,127.8,127.6,126.0,101.7,101.3,99.7,98.8,92.6,78.8,78.4$, 
$77.9,77.3,76.1,75.8,74.9,72.9,70.9,70.8,68.2,67.5,67.2,67.1,66.5,66.4,62.7,57.8$, $55.2,38.1,29.6,21.2,17.1,16.4$.

HR-ESI-MS (m/z): calcd. for $\mathrm{C}_{54} \mathrm{H}_{61} \mathrm{Cl}_{3} \mathrm{~N}_{8} \mathrm{O}_{15} \mathrm{Na}^{+},(\mathrm{M}+\mathrm{Na})^{+}$1189.3214; found, 1189.3215 .

$\mathrm{N}$-benzyloxycarbonyl-3-aminopropanyl-3,6-O-benzyl-2-azido-2-deoxy-4-O-hydroxy)$(1 \rightarrow 4)-\beta$-D-mannopyranosyl uronate)- (2-azido-2-deoxy-3- $O$-acetyl)-(1 $\rightarrow 3)-\alpha$-L-

fucopyranosyl 2 -(trichloroacetylcarbonylamino)-2-deoxy-4- $O$-benzyl- $\beta$-Dfucopyranoside (34):

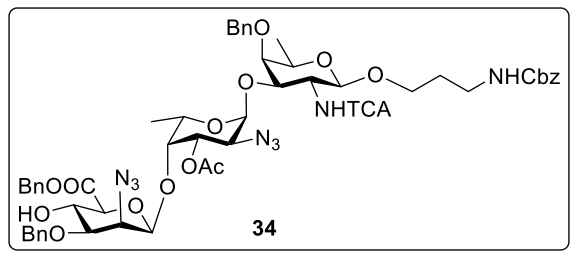

Trisaccharide 33 (65 mg, $0.056 \mathrm{mmol}$ ) was dissolved in $80 \%$ aq. AcOH (5.6 mL) and the resulting solution was heated at $80^{\circ} \mathrm{C}$ for $1 \mathrm{~h}$ (monitored by TLC, 6:4 EtOAc: pet ether). The mixture was concentrated in vacuo and the resulting diol was dissolved in $\mathrm{CH}_{2} \mathrm{Cl}_{2}(5.5 \mathrm{~mL})$ followed by addition of $\mathrm{H}_{2} \mathrm{O}(2 \mathrm{~mL})$, TEMPO (10 mg) and BAIB (36 mg, $\left.0.112 \mathrm{mmol}\right)$. The reaction mixture was stirred at $\mathrm{rt}$ for overnight, (monitored by TLC). The reaction mixture was concentrated in vacuo and kept under high vacuum for benzyl protection of free acid. The crude acid intermediate was dissolved on dry DMF $(1 \mathrm{~mL})$ followed by addition of $\mathrm{BnBr}$ (38 $\mathrm{mg}, 0.23 \mathrm{mmol})$ and $\mathrm{NaHCO}_{3}(50 \mathrm{mg}, 0.18 \mathrm{mmol})$. After $2 \mathrm{~h}$, TLC showed the reaction had gone to completion. The reaction mixture was washed with sat. $\mathrm{NaHCO}_{3}(20 \mathrm{~mL})$. The organic layer was dried over anhydrous $\mathrm{Na}_{2} \mathrm{SO}_{4}$ and concentrated under reduced pressure, and purified by column chromatography on silica gel (4:6 ethyl acetate: pet ether) to afford compound $\mathbf{3 4}$ as a viscous liquid, $44 \mathrm{mg}(69 \%$, over three steps).

$[\alpha]^{25} \mathrm{D}-15.981\left(c=0.10, \mathrm{CHCl}_{3}\right)$.

IR $\left(\mathrm{cm}^{-1}, \mathrm{CHCl}_{3}\right) v 3344,2888,2112,1714,1525,1374,1252,1098,753,699$.

${ }^{1} \mathrm{H}$ NMR (500 MHz, $\left.\mathrm{CDCl}_{3}\right) \delta$ 7.40-7.36 (m, 17H, ArH), 7.34-7.30 (m, 3H, ArH), 5.32-5.20 (m, 2H), 5.18-5.16 (m, 1H, NH), 5.09 (s, 2H, $\mathrm{CH}_{2}$ of Cbz), 5.05-4.99 (m, 2H), 4.87-4.70 (m, $5 \mathrm{H}), 4.49(\mathrm{~s}, 1 \mathrm{H}), 4.22-3.94(\mathrm{~m}, 8 \mathrm{H}), 3.70-3.53(\mathrm{~m}, 4 \mathrm{H}), 3.43-3.24(\mathrm{~m}, 3 \mathrm{H}), 2.04\left(\mathrm{~s}, 3 \mathrm{H}, \mathrm{CH}_{3}\right.$ of OAc), 1.79-1.76 ( m, 2H CH 2 linker), $1.30\left(\mathrm{~d}, J=6.32 \mathrm{~Hz}, 3 \mathrm{H}, \mathrm{CH}_{3}\right), 1.07$ (d, $J=6.56 \mathrm{~Hz}$, $\left.3 \mathrm{H}, \mathrm{CH}_{3}\right)$. 
${ }^{13} \mathrm{C}$ NMR $\left(125 \mathrm{MHz}, \mathrm{CDCl}_{3}\right) \delta 170.5,167.7,162.2,156.5,138.1,137.2,137.2,134.8,128.7$, 128.6, 128.5, 128.3, 128.0, 127.9, 127.7, 100.9, 99.5, 98.8, 92.5, 81.4, 78.7, 78.6, 77.9, 75.5, $75.4,74.9,72.4,70.8,70.3,67.9,67.7,67.3,66.5,66.4,60.7,57.7,55.3,38.1,29.6,20.8$, $17.1,16.5$.

HR-ESI-MS (m/z): calcd. for $\mathrm{C}_{54} \mathrm{H}_{61} \mathrm{Cl}_{3} \mathrm{~N}_{8} \mathrm{O}_{16} \mathrm{Na}^{+},(\mathrm{M}+\mathrm{Na})^{+}$1207.3151; found, 1207.3150.

3-amino-propanyl-2-acetamido-2-deoxy- $\beta$-D-mannopyranosyluronate-( $1 \rightarrow 4)$-2acetamido-2-deoxy-3-Oacetyl- $\alpha$-L-fucopyranosyl-( $1 \rightarrow 3)$-2-acetamido-2-deoxy- $\beta$-Dfucopyranoside (2):

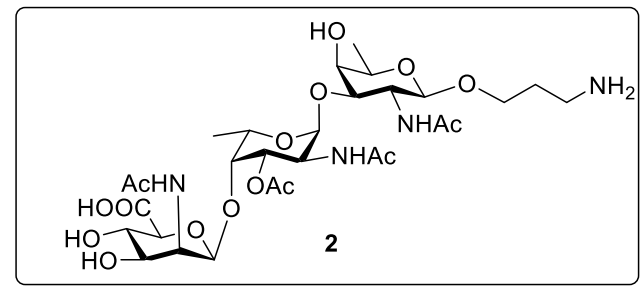

To a solution of $34(10 \mathrm{mg}, 0.01 \mathrm{mmol})$ in THF $(0.5 \mathrm{~mL})$ was added AcOH $(0.8 \mathrm{~mL})$ followed by addition of $\mathrm{Zn}$ dust $(60 \mathrm{mg}$ ) and the reaction mixture was stirred at $\mathrm{rt}$ for $12 \mathrm{~h}$, after which TLC $\left(\mathrm{CH}_{3} \mathrm{OH}\right.$ :EtOAc, 5:95, v/v) showed the transformation had gone to completion. The solution was filtered, and the filtrate was concentrated in vacuo. To avoid the $O$-acetylation, $\mathrm{NH}_{2}$ intermediate was dissolved in $\mathrm{Et}_{3} \mathrm{~N}: \mathrm{MeOH}$ followed by addition of $\mathrm{Ac}_{2} \mathrm{O}(0.1 \mathrm{~mL})$ and the reaction mixture was stirred at $\mathrm{rt}$ for $1 \mathrm{~h}$, after which TLC (EtOAc: Pet ether, 4:6, v/v) showed the completion of reaction. The residue was purified on silica gel affording the NHAc compound as a viscous solid in a quantitative yield. Now triacetamido compound $(10 \mathrm{mg}, 0.01 \mathrm{mmol})$ was dissolved in $\mathrm{EtOH}(1 \mathrm{~mL})$. To this solution $\mathrm{Pd}(\mathrm{OH})_{2} / \mathrm{C}$ (25 mg) was added. The reaction was stirred under hydrogen atmosphere for $10 \mathrm{~h}$. The reaction mixture was filtered through a Celite bed using methanol as eluent. Filtrate was concentrated under reduced pressure and dried under high vacuum to obtain $\mathbf{2}$ as a sticky solid (4 mg, 72\%).

$[\alpha]^{25} \mathrm{D}-11.898\left(c=0.10, \mathrm{CHCl}_{3}\right)$.

IR $\left(\mathrm{cm}^{-1}, \mathrm{CHCl}_{3}\right) v 3432,2521,1652,1461,1219,858,761,513$.

${ }^{1} \mathrm{H}$ NMR (500 MHz, D $\left.2 \mathrm{O}\right) \delta$ 5.03-5.01 (m, 2H), $4.59(\mathrm{~d}, J=4.2 \mathrm{~Hz}, 1 \mathrm{H}), 4.42-4.35(\mathrm{~m}, 4 \mathrm{H})$, 4.21-4.18 (m, 3H), $4.02(\mathrm{~s}, 1 \mathrm{H}), 4.01-3.98(\mathrm{~m}, 3 \mathrm{H}), 3.80-3.56(\mathrm{~m}, 11 \mathrm{H}), 3.09-3.04(\mathrm{~m}, 3 \mathrm{H})$, 
2.12 (s, 3H, OAc), 2.08 (s, 3H, NHAc), 1.99 (s, 6H, NHAc), 1.36 (m, 2H), 1.27 (d, J = 5.32 $\left.\mathrm{Hz}, 3 \mathrm{H}, \mathrm{CH}_{3}\right), 1.24$ (d, $\left.J=6.43 \mathrm{~Hz}, 3 \mathrm{H}, \mathrm{CH}_{3}\right)$.

${ }^{13} \mathrm{C}$ NMR $\left(150 \mathrm{MHz}, \mathrm{D}_{2} \mathrm{O}\right) \delta 174.4,174.3,174.0,173.9,173.7,101.5,99.9,99.0,77.0,71.7$ $70.7,70.4,69.8,69.5,68.0,66.9,62.5,53.1,51.4,47.2,42.3,37.7,30.2,26.7,22.2,22.1$, 15.4, 15.3 .

HR-ESI-MS (m/z): calcd. for $\mathrm{C}_{29} \mathrm{H}_{48} \mathrm{~N}_{4} \mathrm{O}_{16} \mathrm{Na}^{+},(\mathrm{M}+\mathrm{Na})^{+}$731.2958; found, 731.2959. 
Current Data Parameters

NAME SSK-11-AMB-742-1H

$\begin{array}{lr}\text { EXPNO } & 14 \\ \text { PROCNO } & 1\end{array}$

F2 - Acquisition Parameters

Date_ 20170217

$\begin{array}{lr}\text { Time- } & 2017.53 \\ \text { Time } & 23.53\end{array}$

INSTRUM $5 \mathrm{~mm} \mathrm{PABBO}$ sB/

PULPROG $5 \mathrm{~mm}$ PABBO BB/

PULPROG Zg30

TD

NS

NS

SWH

FWH

AQ

$\mathrm{AQ}$
$\mathrm{RG}$
$\mathrm{DW}$

DW

TE

D1 9

$\mathrm{CDCl3}$

16

$10000.000 \mathrm{~Hz}$
$0.152588 \mathrm{~Hz}$

$3.2767999 \mathrm{sec}$

$$
48.36
$$

50.000 usec

6.50 usec

1. $00000000 \mathrm{sec}$

1

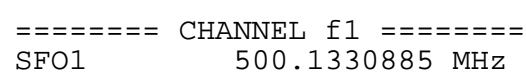

NUC1 $1 \mathrm{H}$

P1 13.00 usec

PLW1 $13.00000000 \mathrm{~W}$

F2 - Processing parameters

SI $\quad 65536$

SF $\quad 500.1300000 \mathrm{MHz}$

WDW EM

$\begin{array}{lll}\text { SSB } & 0 & 0.30 \mathrm{~Hz}\end{array}$

$\begin{array}{lll}\text { GB } & 0 & 1.00\end{array}$

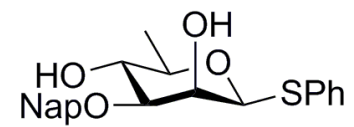

12

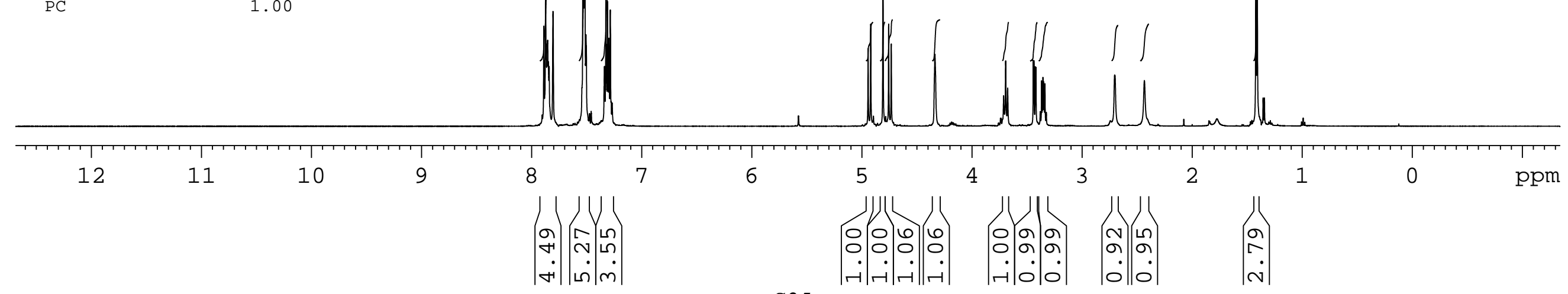




\section{Current Data Parameters \\ NAME SSK-11-AMB-742-13C \\ EXPNO \\ PROCNO \\ 15
1}

F2 - Acquisition Parameters

Date_ 20170217

Time

23.55

INSTRUM

PROBHD

PULPROG

$5 \mathrm{~mm}$ PABBO BB/

TD Zgpg30

SOLVENT 65536

SOLVENT CDCl3

NS

100
0

DS

FIDRES

$A Q$

$\mathrm{RG}$

DW

TE

D1

D11

TDQ $\begin{array}{ll}\text { SF01 } & 125.7703637 \mathrm{MHZ}\end{array}$

NUC1 13C

$\begin{array}{lr}\text { P1 } & 8.90 \text { usec } \\ \text { PLW1 } & 103.00000000 \mathrm{~W}\end{array}$

PLW1

$=======$ CHANNEL $\mathrm{f2}======$

SF02 $500.1320005 \mathrm{MHZ}$

NUC2 $1 \mathrm{H}$

waltz16

$\begin{array}{lr}\text { PCPD2 } & 80.00 \text { usec } \\ \text { PLW2 } & 13.00000000 \mathrm{~W}\end{array}$

PLW2 13.00000000 W

$\begin{array}{ll}\text { PLW12 } & 0.34327999 \mathrm{~W} \\ \text { PLW13 } & 0.17267001 \mathrm{~W}\end{array}$

F2 - Processing parameters

SI $\quad 32768$

SF $\quad 125.7577676 \mathrm{MHz}$

$\begin{array}{lll}\text { WDW } & & \\ \text { SSB } & 0 & \text { EM }\end{array}$

$\begin{array}{lll}\text { SSB } & 0 & 1.00 \mathrm{~Hz} \\ \text { LB } & & \end{array}$

$\begin{array}{lll}\text { GB } & 0 & 1.40\end{array}$
の . . . . . . . . ল

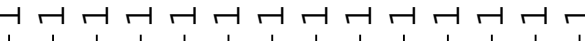

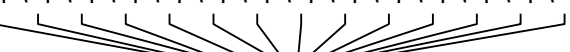

o N
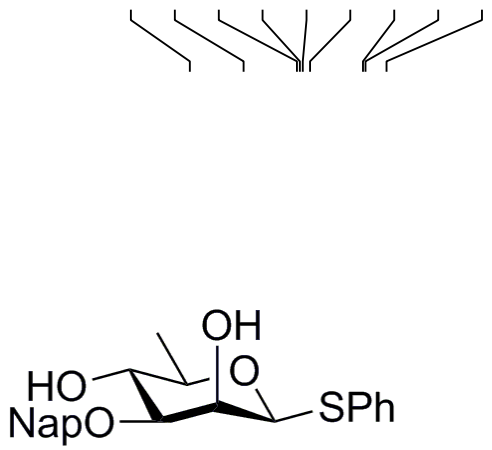

12 


\section{SSK-20-BKG-203-DEP}

\begin{tabular}{|c|c|}
\hline \multirow{2}{*}{\multicolumn{2}{|c|}{$\begin{array}{l}\text { Current Data Parameters } \\
\text { NAAME } \\
\text { EXPNO-20-BKG-203-DEPT }\end{array}$}} \\
\hline & \\
\hline $\begin{array}{l}\text { F2 - Acqui } \\
\text { Date } \\
\text { Time } \\
\text { INSTRUM } \\
\text { PROBHD } \\
\text { PULPROG } \\
\text { TD } \\
\text { SOLVENT } \\
\text { NS } \\
\text { DS } \\
\text { SWH } \\
\text { FIDRES } \\
\text { AQ } \\
\text { RG } \\
\text { DW } \\
\text { DE } \\
\text { TE } \\
\text { CNST2 } \\
\text { D1 } \\
\text { D2 } \\
\text { D12 } \\
\text { TD0 }\end{array}$ & $\begin{array}{c}\text { isition Parameters } \\
20180311 \\
5.03 \\
\text { spect } \\
5 \text { mm PABBO BB- } \\
\text { dept135 } \\
65536 \\
\text { CDCl3 } \\
38 \\
0 \\
24038.461 \mathrm{~Hz} \\
0.366798 \mathrm{~Hz} \\
1.3631488 \mathrm{sec} \\
2050 \\
20.800 \mathrm{usec} \\
6.50 \text { usec } \\
295.4 \mathrm{~K} \\
145.0000000 \\
1.00000000 \mathrm{sec} \\
0.00344828 \mathrm{sec} \\
0.00002000 \mathrm{sec} \\
1\end{array}$ \\
\hline $\begin{array}{l}\text { =E====== } \\
\text { NUC1 } \\
\text { P1 } \\
\text { P2 } \\
\text { PL1 } \\
\text { PL1W } \\
\text { SFO1 }\end{array}$ & $\begin{array}{l}=\text { CHANNEL } \mathrm{f} 1======= \\
13 \mathrm{C} \\
8.50 \text { usec } \\
17.00 \text { usec } \\
-2.00 \mathrm{~dB} \\
56.53121948 \mathrm{~W} \\
100.6228298 \mathrm{MHz}\end{array}$ \\
\hline $\begin{array}{l}\text { ======" } \\
\text { CPDPRG } 2 \\
\text { NUC2 } \\
\text { P3 } \\
\text { P4 } \\
\text { PCPD2 } \\
\text { PL2 } \\
\text { PL12 } \\
\text { PL2W } \\
\text { PL12W } \\
\text { SFO2 }\end{array}$ & $\begin{array}{c}\text { CHANNEL f2 }===== \\
\text { C } \begin{array}{c}\text { Waltz16 } \\
1 \mathrm{H}\end{array} \\
14.75 \mathrm{usec} \\
29.50 \mathrm{usec} \\
80.00 \mathrm{usec} \\
-1.00 \mathrm{~dB} \\
13.69 \mathrm{~dB} \\
10.56200695 \mathrm{~W} \\
0.35871249 \mathrm{~W} \\
400.1316005 \mathrm{MHz}\end{array}$ \\
\hline $\begin{array}{ll}\text { F2 - Proce } \\
\text { SI } \\
\text { SF } & 10 \\
\text { WDW } & \\
\text { SSB } & 0 \\
\text { LB } & \\
\text { GB } & 0 \\
\text { PC } & \end{array}$ & $\begin{array}{c}\text { essing parameters } \\
32768 \\
100.6127690 \mathrm{MHz} \\
\mathrm{EM} \\
3.00 \mathrm{~Hz} \\
1.40\end{array}$ \\
\hline
\end{tabular}

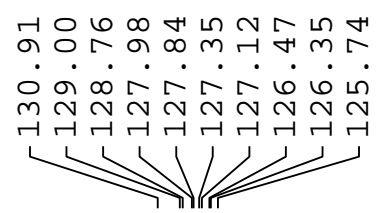

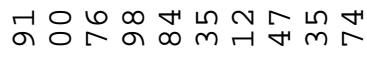

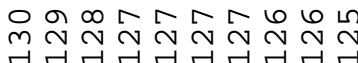

Time 5.03

PROBHD $5 \mathrm{~mm}$ PABBO BB

PULPROG dept13

SOLVENT ${ }_{38} \mathrm{CDCl}$

$24038.461 \mathrm{~Hz}$

AQ $1.3631488 \mathrm{sec}$

DW $\quad 20.800$ usec

TE $\quad 295.4 \mathrm{~K}$

1.00000000

D12 $0.00002000 \mathrm{sec}$

$=======$ CHANNEL $\mathrm{f} 1=======$

$\begin{array}{ll}\text { P1 } & 8.50 \text { usec } \\ \text { P2 } & 17.00 \text { usec }\end{array}$

PL1W 56.53121948 W

$======$ CHANNEL $12=======$

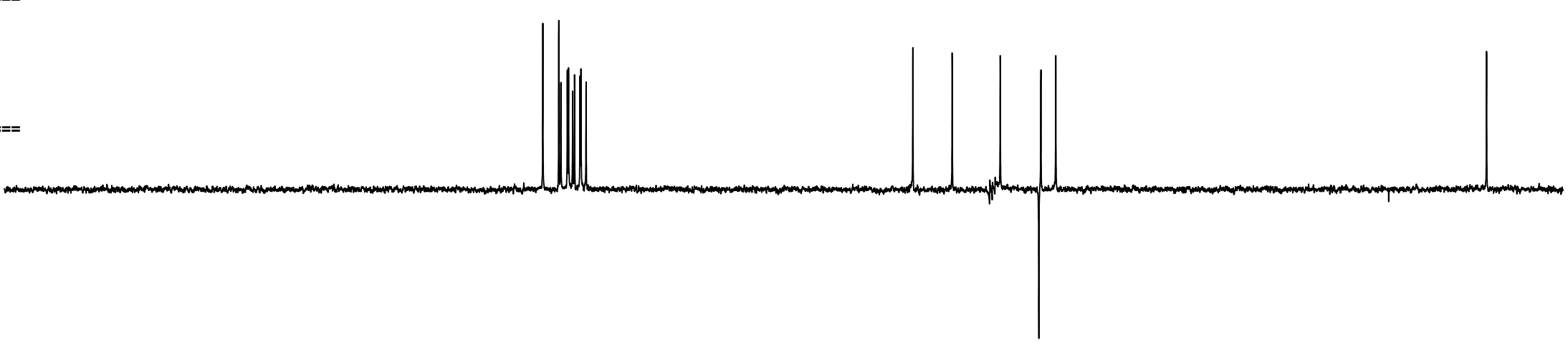

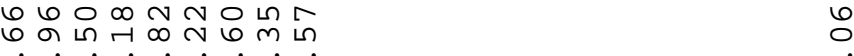

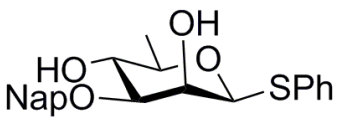

12 
SSK-11 - AMB - 742 - HHCOSY

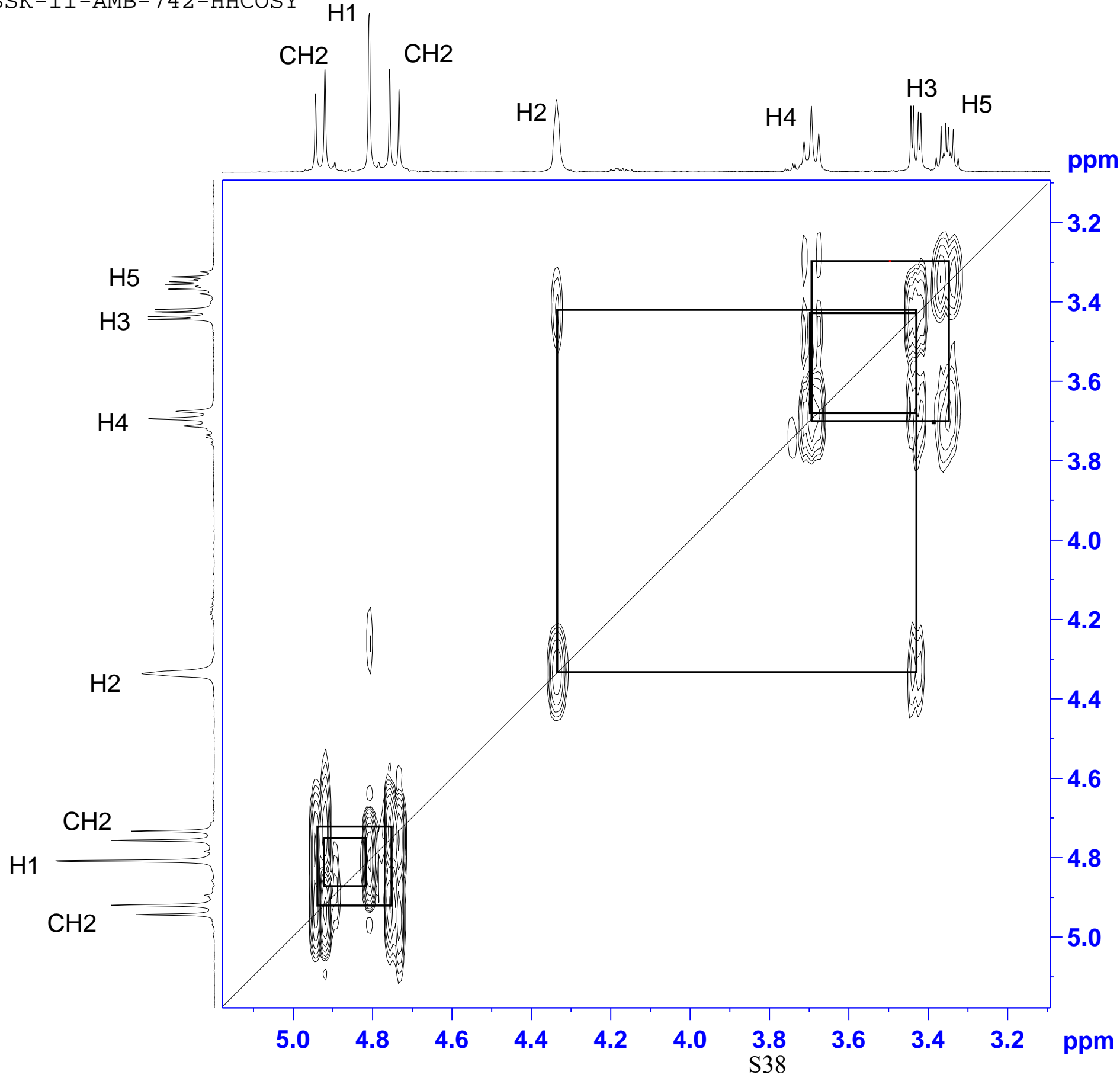

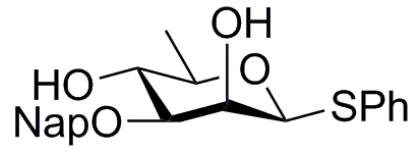

12

Current Data Parameters
NAME SSK-11-AMB-742-HHCOSY

EXPNO $\quad 16$

F2 - Acquisition Parameters

Date ${ }_{\text {Time }}$

23.58
spect

$5 \mathrm{~mm}$ PABBO BB

PULPROG COSygpppq

TD SOLVENT

NS

$\begin{array}{lr}\text { DS } & \oplus \\ \text { SWH } & 3605.769 \mathrm{~Hz} \\ \text { FIDRES } & 1.760630 \mathrm{~Hz}\end{array}$

AQ $\quad 0.2839893 \mathrm{sec}$

$\begin{array}{lr}\text { RG } & 134.65 \\ \text { DW } & 138.667 \text { usec }\end{array}$

$\begin{array}{lr}\text { DE } & 6.50 \text { usec } \\ \text { TE } & 299.0 \mathrm{~K} \\ \text { DQ } & 0.00000300 \mathrm{sec}\end{array}$

$\begin{array}{ll}\text { D0 } & 0.00000300 \mathrm{sec} \\ \text { D1 } & 1.00000000 \mathrm{sec}\end{array}$

$\begin{array}{ll}\text { D1 } & 1.00000000 \mathrm{sec} \\ \text { D11 } & 0.03000000 \mathrm{sec} \\ \text { D12 } & 0.0002000 \mathrm{sec}\end{array}$

$\begin{array}{ll}\text { D12 } & 0.00002000 \text { sec } \\ \text { D13 } & 0.00000400 \text { sec } \\ \text { D16 } & 0.00020000 \text { sec }\end{array}$

INO $\quad 0.00027740 \mathrm{sec}$

$========$
CHANNEL $\mathrm{f} 1 \quad========$
$500.1323239 \mathrm{MHz}$

$\begin{array}{ll}\text { SF01 } & 500.1323239 \mathrm{MHZ} \\ \text { NUC1 } & 1 \mathrm{H}\end{array}$

$\begin{array}{lr}\text { P1 } & 13.00 \text { usec } \\ \text { P17 } & 13.00 \text { usec } \\ \text { P17 } & 2500.00 \text { usec }\end{array}$

$\begin{array}{lr}\text { PLW1 } & 13.000000000 \mathrm{~W} \\ \text { PLW10 } & 2.44109988 \mathrm{~W}\end{array}$

$=====$ GRADIENT CHANNEL $====$

GPNAM[1] SMSQ10.100

$\begin{array}{ll}\text { GPZ1 } & 10.00 \% \\ \text { P16 } & 1000.00 \text { usec }\end{array}$

F1 - Acquisition parameters

TD $\quad 500.1323 \mathrm{MHz}$

$\begin{array}{lr}\text { SF01 } & 500.1323 \mathrm{MHz} \\ \text { FIDRES } & 133.514908 \mathrm{~Hz} \\ \text { SW } & 7.208 \mathrm{ppm}\end{array}$

FnMODE

QF $208 \mathrm{ppm}$

F2 - Processing parameters

$\begin{array}{ll}\text { SI } & 1024 \\ \text { SF } & 500.1300000 \mathrm{MHz}\end{array}$

$\begin{array}{lc}\text { SF } & 500.1300000 \\ \text { WDW } & \text { QSINE }\end{array}$

$\begin{array}{lll}\mathrm{SSB} & \bullet \\ \mathrm{LB} & \bullet \mathrm{Hz}\end{array}$

$\begin{array}{lll}\mathrm{GB} & \mathrm{O} & \\ \mathrm{PC} & & \end{array}$

F1 - Processing parameters

$\begin{array}{lc}\text { MC2 } & \text { QF } \\ \text { SF } & 500.1300000 \\ \text { WHz }\end{array}$

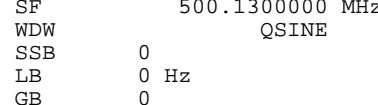


SSK - 11-AMB - 1110-1H

Current Data Parameters

NAME SSK-11-AMB-1110-1H

EXPNO 9

PROCNO

F2 - Acquisition Parameters

$\begin{array}{lr}\text { Date_- } & 20180626 \\ \text { Time } & 17.49\end{array}$

INSTRUM spect

PROBHD $5 \mathrm{~mm}$ PABBO BB/

PULPROG
zg30

$\begin{array}{ll}\text { TD } & 65536 \\ \text { SOLVENT } & \text { CDCl3 }\end{array}$

$\begin{array}{lr}\text { NS } & \text { CDC13 } \\ \text { NS } & 45 \\ \text { DS } & 0\end{array}$ 45
9
0
09

SWH $\quad 10000.000 \mathrm{~Hz}$

$0.152588 \mathrm{~Hz}$

AQ $\quad 3.2767999 \mathrm{sec}$

DW $\quad 50.000$ usec

DE $\quad 6.50$ use

D1 $1.0000000 \% \mathrm{sec}$

TD० 1

$=======$ CHANNEL $\mathrm{f} 1========$
SF01 $500.1330885 \mathrm{MHz}$

$1 \mathrm{H}$

P1 13.35 use

PLW1 $16.00000000 \mathrm{~W}$

F2 - Processing parameters

$\begin{array}{lr}\text { SI } & 65536 \\ \text { SF } & 500.1300137 \mathrm{MHz}\end{array}$

$\begin{array}{lr}\text { SF } & 500.1300137 \mathrm{MHz} \\ \text { WDW } & \text { EM }\end{array}$

$\begin{array}{lll}\text { SSB } & 0 & 0.30 \mathrm{~Hz} \\ \mathrm{LB} & 0 & 1.00 \\ \mathrm{PC} & & \end{array}$

$\begin{array}{lll}\text { SSB } & 0 & 0.30 \mathrm{~Hz} \\ \mathrm{LB} & 0 & 1.00 \\ \mathrm{PC} & & \end{array}$

10

9

8

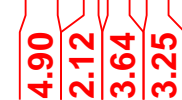

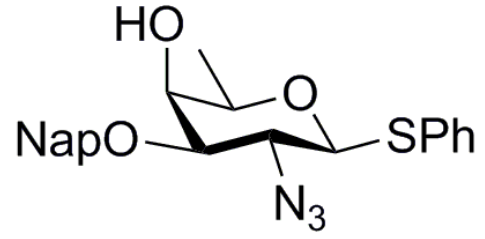

13

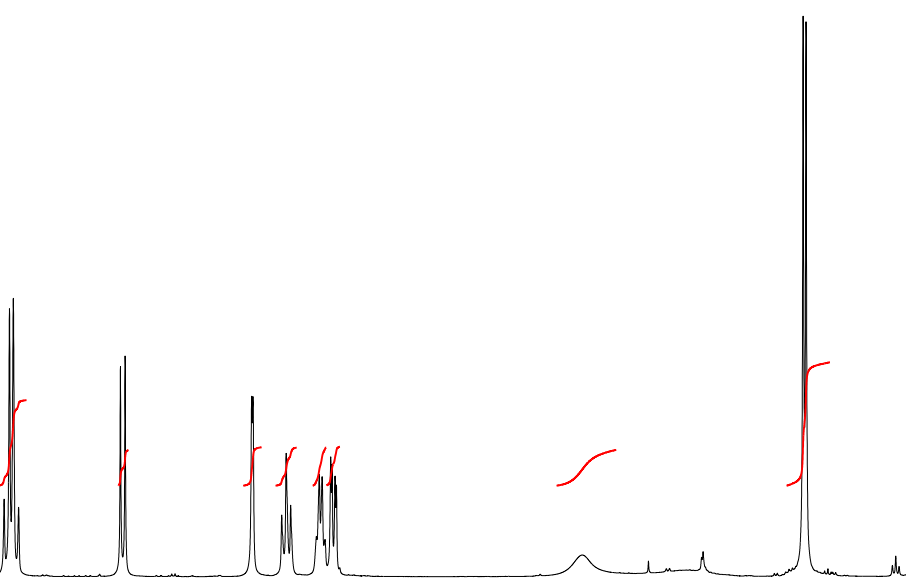

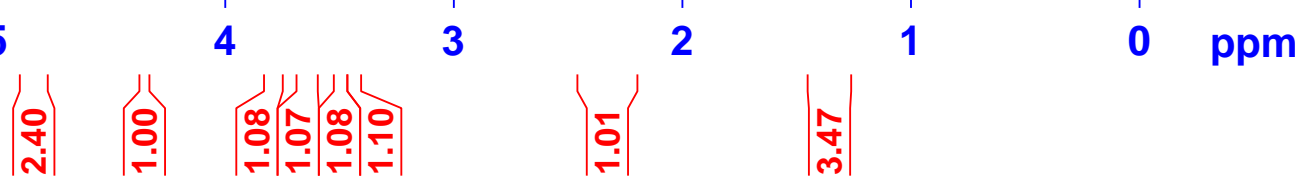


SSK-11-AMB - 1110-13C

Current Data Parameters

NAME SSK-11-AMB-1110-13C

EXPNO

EXPNO

5
1

F2 - Acquisition Parameters

\begin{tabular}{lr} 
Date__ & 20180628 \\
\hline
\end{tabular}

4.01
spect

PROBHD $5 \mathrm{~mm}$ PABBO BB-

$\begin{array}{lr}\text { PULPROG } & \text { zgpg30 } \\ \text { TD } & 65536\end{array}$

$\begin{array}{ll}\text { TD } & 65536 \\ \text { SOLVENT } & \text { CDCl3 }\end{array}$

NS

DS

SWH

FIDRES

AQ

$\mathrm{RG}$
$\mathrm{DW}$
$\mathrm{DE}$

TE

D1

D11

$26041.666 \mathrm{~Hz}$

$0.397364 \mathrm{~Hz}$

$1.2582912 \mathrm{sec}$

2050

.200 usec
6.50 usec $295.7 \mathrm{~K}$

$1.0000000 \odot \mathrm{sec}$

$0.0300000 \mathrm{sec}$

$=======$ CHANNEL $\mathrm{f} 1$

NUC1

P1

PL1

PL1W

$1====$

$13 \mathrm{C}$
8.50 usec

$-2.00 \mathrm{~dB}$

$56.53121948 \mathrm{~W}$

$=======$ CHANNEL f2 $=======$
CPDPRG 2 walt CPDPRG

PCPD2

waltz16

PL12

PL13

PL2W

PL12W

PL13W

SF02

F2 - Processing parameters

SI 32768

SF $\quad 100.6127473 \mathrm{MHz}$

WDW

LB

GB

$1.00 \mathrm{~Hz}$

$-1.00 \mathrm{~dB}$

$13.69 \mathrm{~dB}$

$10.56200695 \mathrm{~W}$

0.35871249

EM

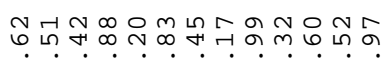

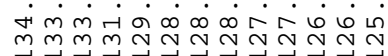

$\longrightarrow$

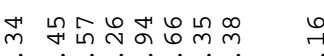

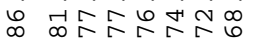

VII

तु

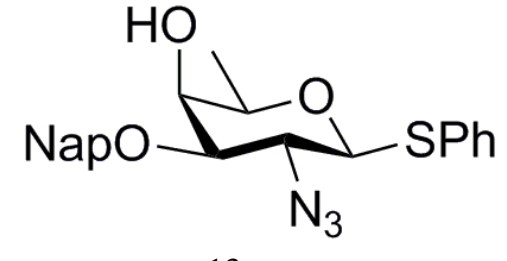

13

$\begin{array}{lllll}200 & 190 & 180 & 170 & 160\end{array}$

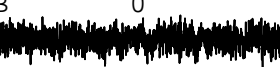

$160 \quad 150$

140

$130 \quad 120$

$110 \quad 100$ 


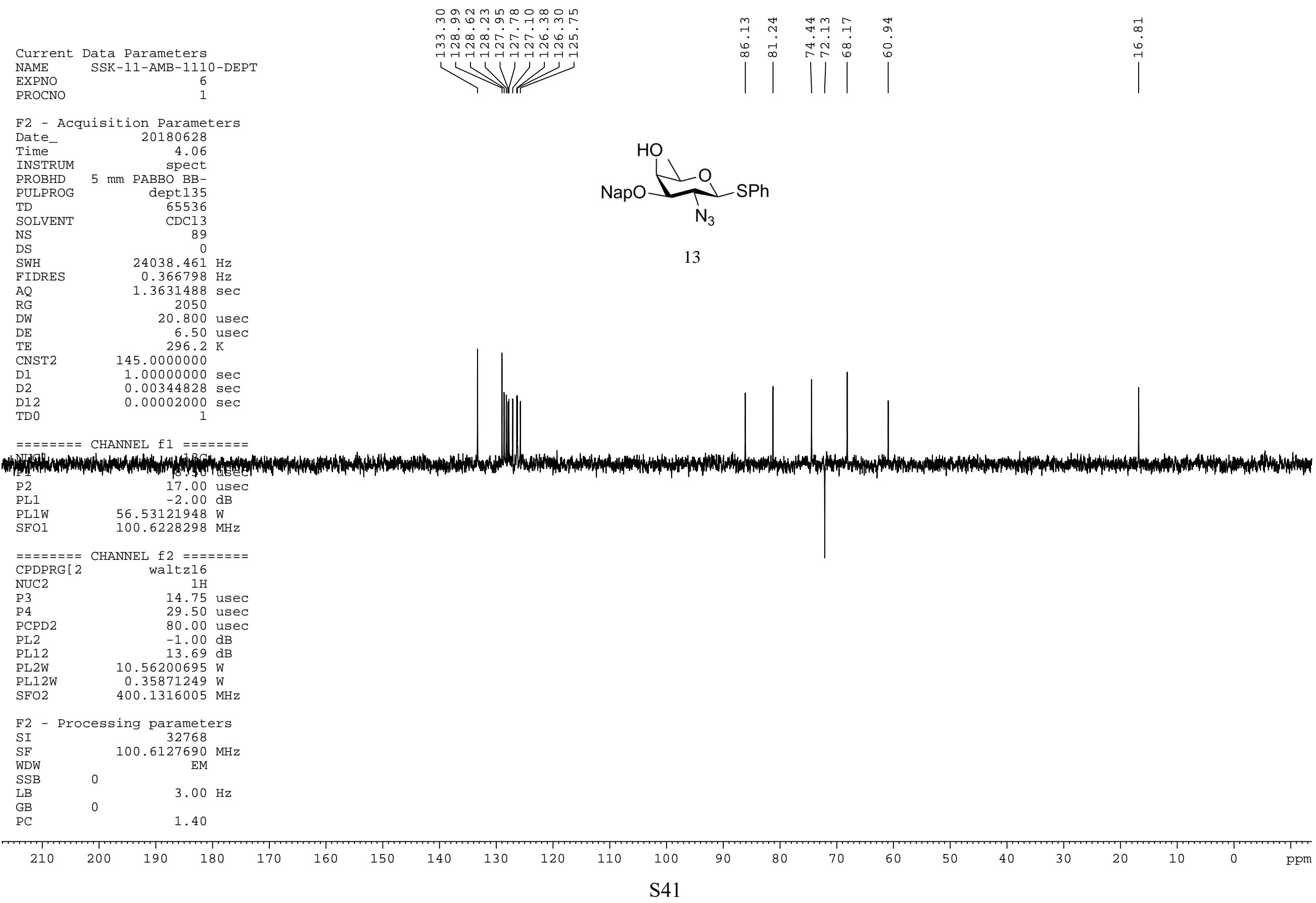


SSK - 11 - AMB - 1110 - HHCOSY

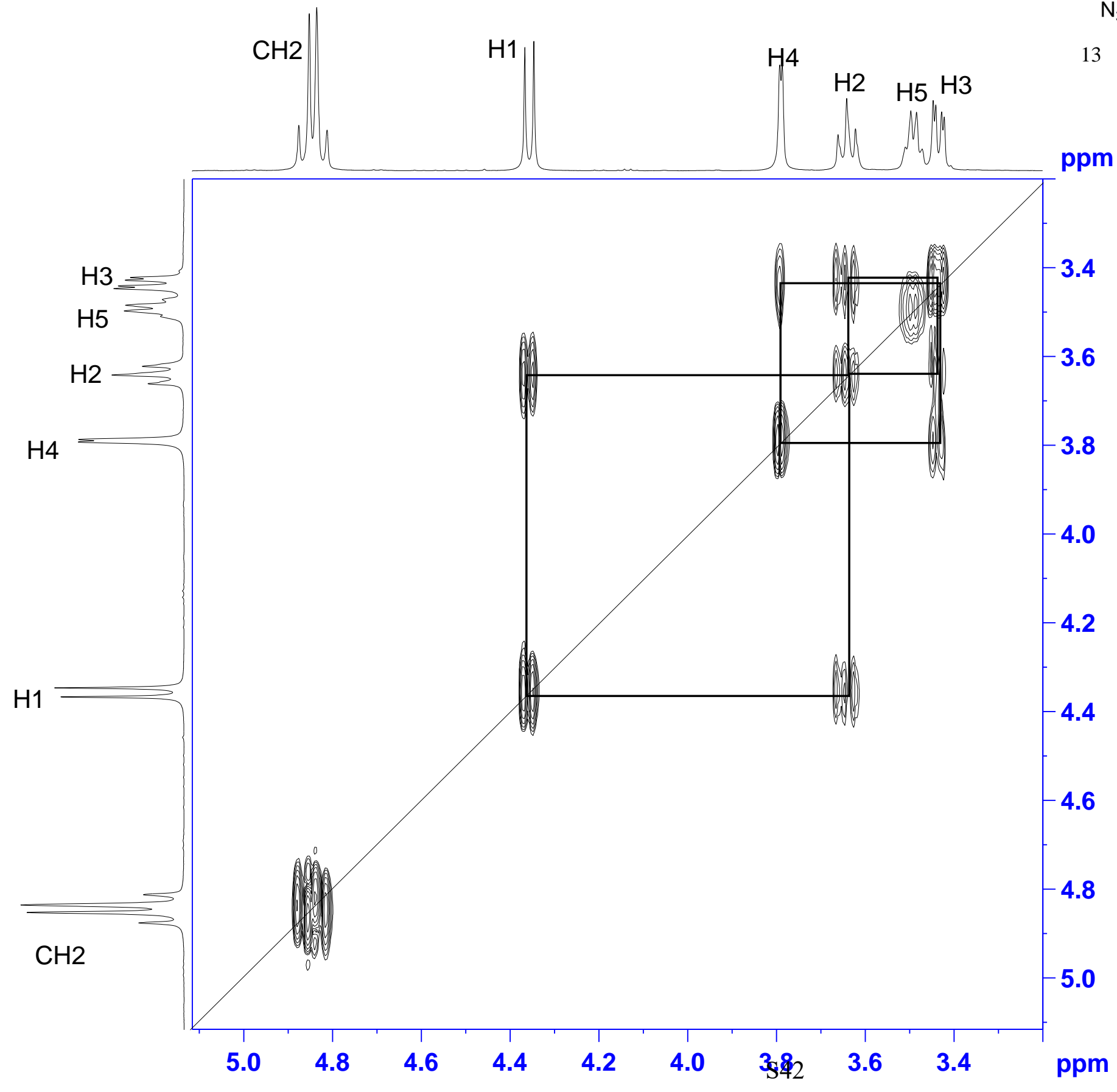

Current Data Parameters
NAME SSK-11-AMB-1110-HHCOSY

EXPNO
PROCNO

F2 - Acquisition Parameters

$\begin{array}{lr}\text { F2 - } & \text { Acquisition Parame } \\ \text { Date } & \text { 20180626 } \\ \text { Time } & 17.52\end{array}$

INSTRUM

PROBHD $5 \mathrm{~mm}$ PABBO BB

PULPROG

SOLVEN

NS

$\begin{array}{lr}\text { DS } & 0 \\ \text { SWH } & 2032.520 \\ \text { FIDRES } & 0.992442 \text { Hz } \\ \text { AO } & 0.5038080 \text { sec } \\ \text { RG } & \end{array}$

$\begin{array}{ll}\text { AQ } & 0.992442 \mathrm{~Hz} \\ \text { RG } & 0.5038080 \mathrm{sec}\end{array}$

$\begin{array}{rr}\text { DW } & 246.000 \text { usec } \\ \text { DE } & 6.50 \text { usec }\end{array}$

TE $\quad 297.3 \mathrm{~K}$

$\begin{array}{ll}\text { DO } & 0.00000300 \mathrm{sec} \\ \text { D1 } & 1.00000000 \mathrm{sec}\end{array}$

$\begin{array}{ll}\text { D1 } & 1.00000000 \mathrm{sec} \\ \text { D11 } & 0.03000000 \mathrm{sec} \\ \text { D12 } & 0.00002000 \mathrm{sec} \\ \text { D13 } & 0.00000400 \mathrm{sec}\end{array}$

$0.00020000 \mathrm{sec}$
$0.00049200 \mathrm{sec}$

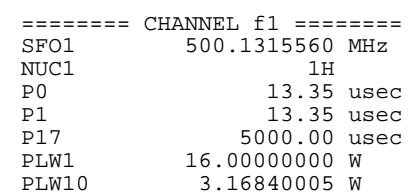

$\begin{array}{ll}\text { PLW1 } & 16.00000000 \mathrm{~W} \\ \text { PLW10 } & 3.16840005 \mathrm{~W}\end{array}$

$======$ GRADIENT CHANNEL $====$

GPNAM[1] SMSQ10.100

$\begin{array}{lc}\text { GPZ1 } & 10.00 \% \\ \text { P16 } & 1000.00 \text { usec }\end{array}$

F1 - Acquisition parameters

TD $\quad 45 \mathrm{Me0.1316} \mathrm{MHz}$

$\begin{array}{lr}\text { TF01 } & 500.1316 \mathrm{MHZ} \\ \text { FIDRES } & 90.33236 \mathrm{~Hz} \\ \text { SW } & 4.064 \mathrm{ppm} \\ \text { FnMODE } & \text { QF }\end{array}$

F2 - Processing parameters

$\begin{array}{ll}\text { SI } & 1024 \\ \text { SF } & 500.1300137 \\ \text { MHz }\end{array}$

WDW QSINE

$\begin{array}{ll}\text { SSB } & \oplus \\ \text { LB } & \oplus \mathrm{Hz} \\ \text { GB } & \oplus\end{array}$

$\begin{array}{lll}\mathrm{GB} & \mathrm{P} & 1.40\end{array}$

F1 - Processing parameters

SI 21024

$\begin{array}{lr}\text { MC2 } & 500.1300137 \mathrm{QHZ} \\ \text { SF } & \text { QSINE }\end{array}$

$\begin{array}{ll}\text { WDW } & \\ \text { SSB } & 0 \\ \text { LB } & \oplus \mathrm{Hz} \\ \text { GB } & 0\end{array}$ 
Current Data Parameters

NAME SSK-11-AMB-1111-1H

EXPNO

8

F2 - Acquisition Parameters

Date_ 20180628

Time 4.20

spect
INSTRUM $5 \mathrm{~mm}$ PABBO BB-

PROBHD $5 \mathrm{~mm}$ PABBO BB-
PULPROG
zg30

$\begin{array}{lr}\text { PULPROG } & \mathrm{Zg} 30 \\ \text { TD } & 54274\end{array}$

SOLVENT CDCl3

NS

16
0

SWH $\quad 8223.685 \mathrm{~Hz}$

FIDRES $\quad 0.151522 \mathrm{~Hz}$

$\mathrm{AQ} \quad 3.2998593 \mathrm{sec}$

RG

DW

DE $\quad 60.800$ usec

$\begin{array}{ll}6.50 & \text { usec } \\ \text { TE } & 295.4 \mathrm{~K}\end{array}$

D1 $\quad 1.00000000 \mathrm{sec}$

TDO

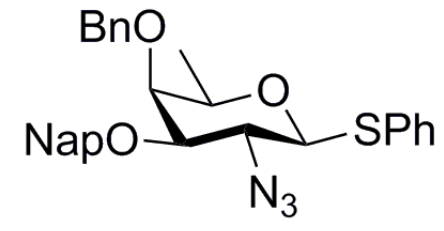

14

$\begin{array}{lr}=======\text { CHANNEL } \mathrm{f} 1======= \\ \text { NUC1 } & 1 \mathrm{H} \\ \text { P1 } & 14.75 \mathrm{usec} \\ \text { PL1 } & -1.00 \mathrm{~dB} \\ \text { PL1W } & 10.56200695 \mathrm{~W} \\ \text { SF01 } & 400.1324710 \mathrm{MHz}\end{array}$

F2 - Processing parameters

SI 32768

SF $400.1300095 \mathrm{MHz}$

WDW

SSB $\odot$

LB

$0.30 \mathrm{~Hz}$

PC

1.00
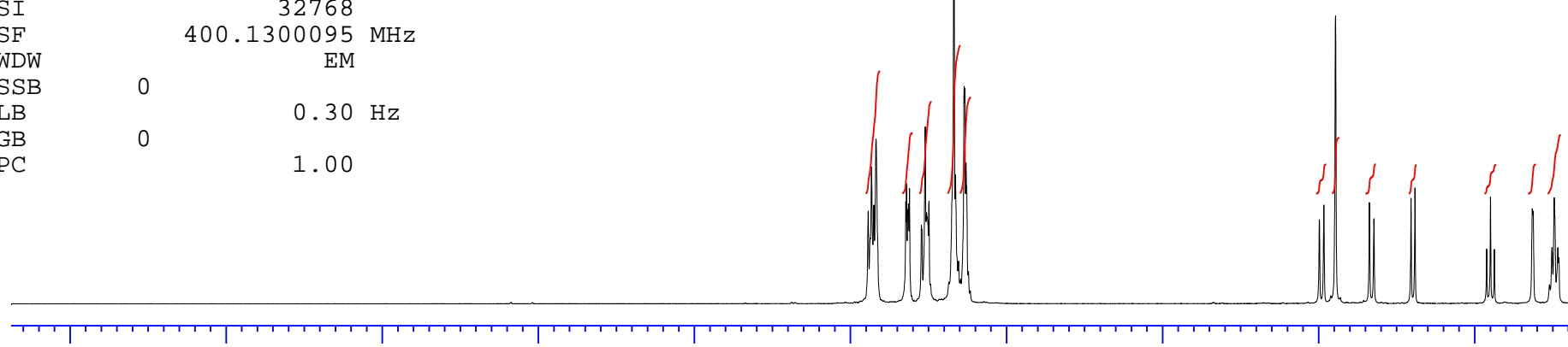

13

12

11

10

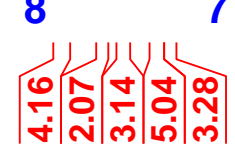

7

6

3

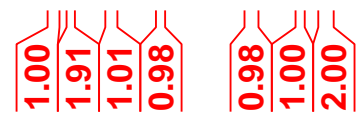

0 ppm 
SSK - 11 - AMB - 1111 - 13C

Current Data Parameters

NAME SSK-11-AMB-1111-13C PROCNO 1

F2 - Acquisition Parameters Date_ 20180518 Time 20180518
5.48

INSTRUM

PROBHD $5 \mathrm{~mm}$ PABBO BB

PULPROG $\quad$ zgpg30

TD 65536

SOLVENT CDCI3

NS

26
4

SWH 26041.666 Hz

FIDRES $\quad 0.397364 \mathrm{~Hz}$

AQ $\quad 1.2582912 \mathrm{sec}$

$\begin{array}{lr}\text { RG } & 2050 \\ \text { DW } & 19.200 \text { use }\end{array}$

19.200 usec
6.50 usec

$\begin{array}{lr}\text { TE } & 297.2 \mathrm{~K} \\ \text { D1 } & 1.00000000 \mathrm{sec}\end{array}$

$\begin{array}{lr}\text { D11 } & 0.03000000 \mathrm{sec} \\ \text { TD0 } & 1\end{array}$

$========$ CHANNEL f1 ======== NUC1

P1

PL1 $13 \mathrm{C}$ PL1W $56.53121948 \mathrm{~W}$

$========$ CHANNEL f2 ========

$\begin{array}{lr}\text { CPDPRG }[2 & \text { waltz16 } \\ \text { NUC2 } & 1 \mathrm{H}\end{array}$

$\begin{array}{ll}\text { NCC2 } & \text { 80. } \\ \text { PCPD } 20 \text { usec }\end{array}$

$\mathrm{PL} 2$

PL12

PL13

PL2W

PL12W

PL13W

$13.69 \mathrm{~dB}$

$13.69 \mathrm{~dB}$
$14.50 \mathrm{~dB}$

$10.56200695 \mathrm{~W}$

$0.35871249 \mathrm{~W}$

$0.29767781 \mathrm{~W}$

$400.1316005 \mathrm{MHz}$

F2 - Processing parameters

$\begin{array}{lr}\text { SI } & 32768 \\ \text { SF } & 100.6127730 \mathrm{MHZ}\end{array}$

WDW

SSB $\quad 0$

(GB

PC

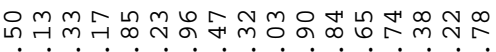

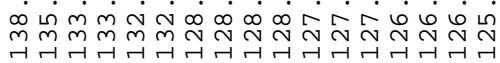

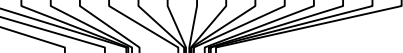

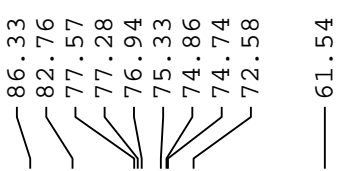

$\stackrel{m}{q}$

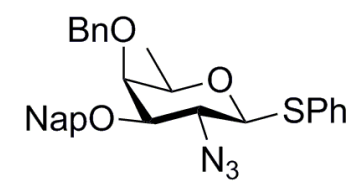

14

200

190

180

170

160

150

140

130

120

110

100

90

80

70

60

50

40

30

20

$\odot \mathrm{ppm}$ 


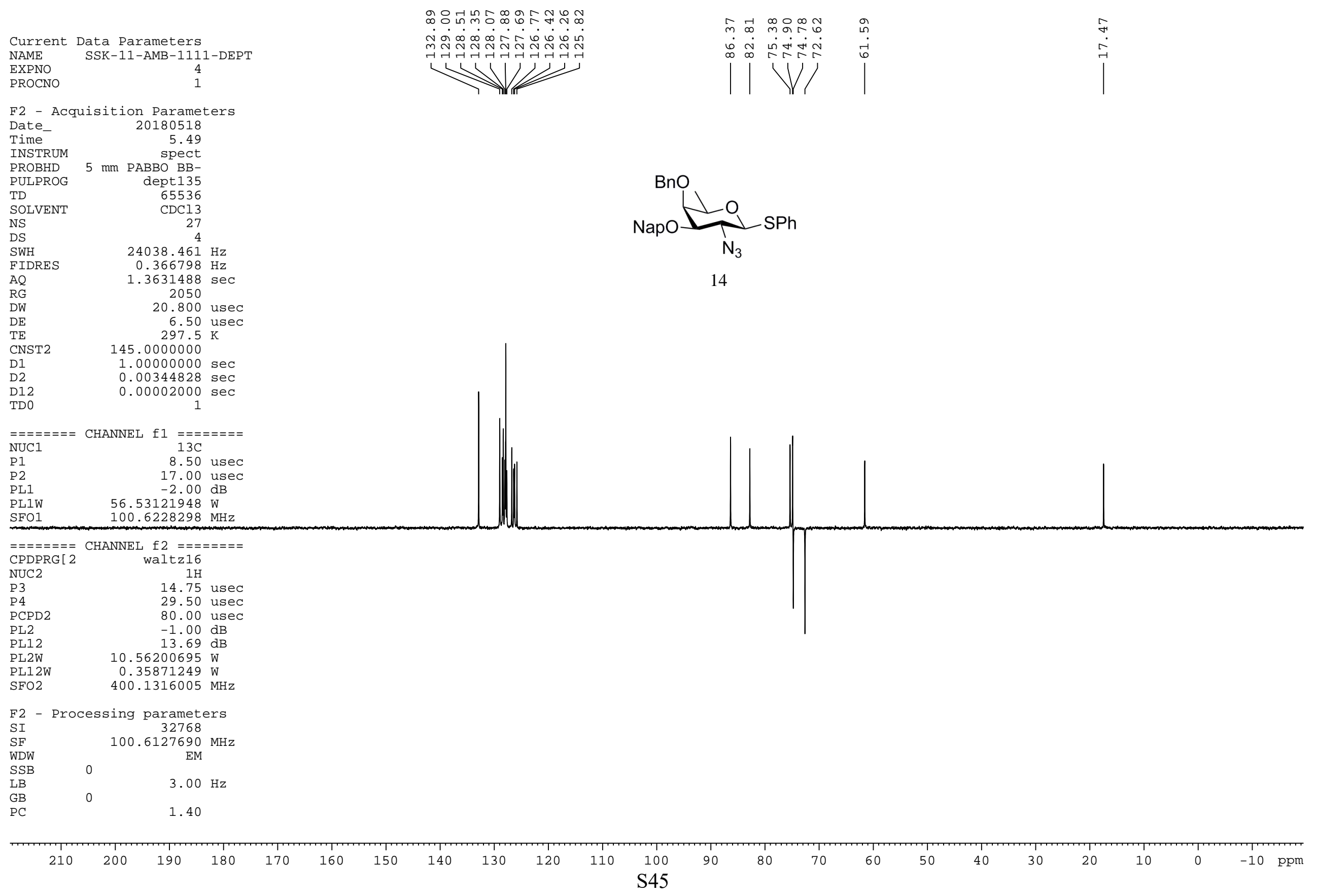




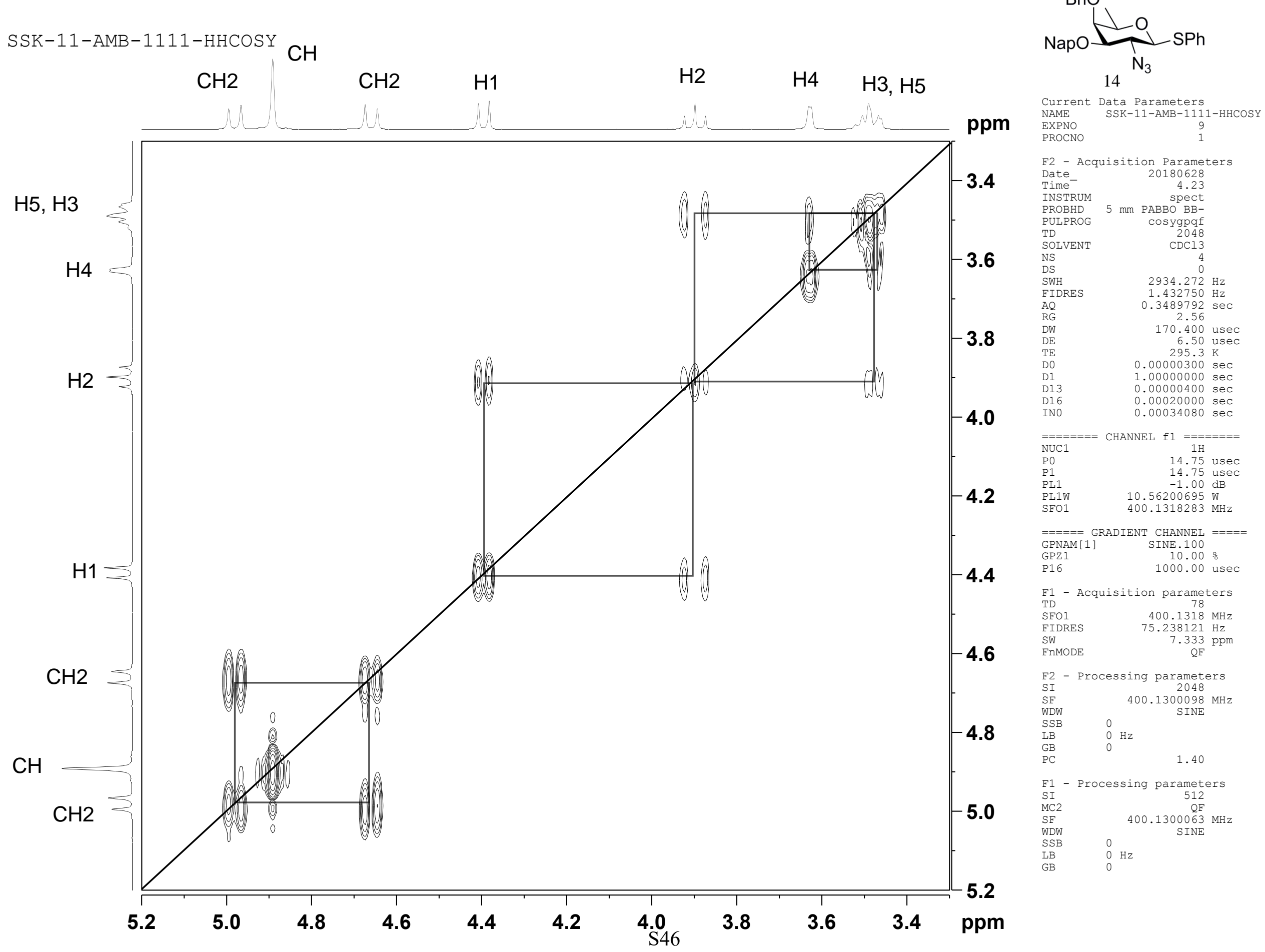


SSK-11-AMB-1114-1H

Current Data Parameters

NAME SSK-11-AMB-1118-1H

PROCNO

6
1

F2 - Acquisition Parameters

Date_ 20180712

$\begin{array}{ll}\text { Time } & 4.01\end{array}$

INSTRUM spect

PROBHD $5 \mathrm{~mm}$ PABBO BB-

PULPROG $\quad \mathrm{zg} 30$

$\begin{array}{ll}\text { TD } & 54274 \\ \text { SOLVENT } & \text { CDC13 }\end{array}$

$\mathrm{CDCl} 3$
7

DS

SWH

FIDRES

FIDRE
RG
DW

DE

TE

TDO

$8223.685 \mathrm{~Hz}$

$0.151522 \mathrm{~Hz}$

$3.2998593 \mathrm{sec}$

60.114

6.50 usec

$295.6 \mathrm{~K}$

$1.0000000 \odot \mathrm{sec}$

$=======$ CHANNEL $\mathrm{f} 1$ ========

NUC1

P1

PL1

PL1W

14.75 usec

$-1.00 \mathrm{~dB}$

$400.1324710 \mathrm{MHz}$

F2 - Processing parameters

SI 32768

SF $\quad 400.1300105 \mathrm{MHz}$

$\begin{array}{lll}\text { WDW } & & \text { EM } \\ \text { SSB } & 0\end{array}$

LB $\quad 0.30 \mathrm{~Hz}$

$\begin{array}{lll}\mathrm{GB} & \odot & 1.00\end{array}$

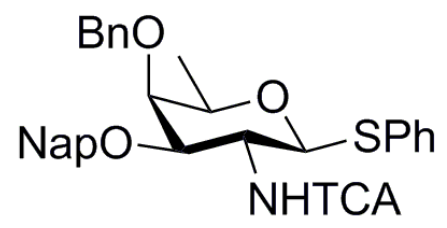

9

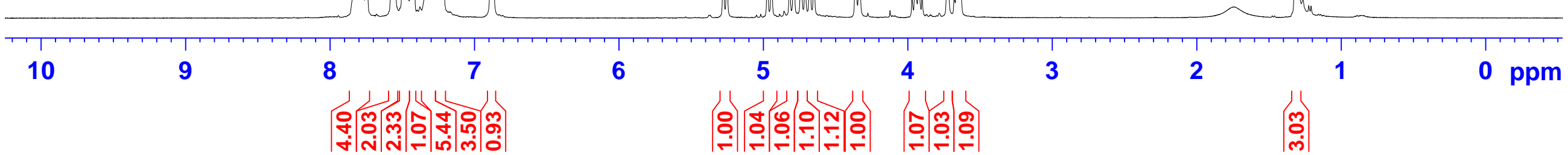


SSK-11-AMB - $1114-13 C$

Current Data Parameters

NAME SSK-11-AMB-1118-13C

EXPNO

PROCNO

1

F2 - Acquisition Parameters

Date 20180712

Time

20180712
4.01

INSTRUM spect

PULPROG 5 TIm PABBO BB-

TD

zgpg 30
65536

SOLVENT

NS
SWH

FIDRES

AQ

RQ

DW

DE

TE

D11

TDQ

$=======$ CHANNEL $\mathrm{f} 1$ NUC1

$\mathrm{P} 1$

8.50
PL1 usec

PL1W $56.53121948 \mathrm{~W}$

$100.6238364 \mathrm{MHz}$
$\mathrm{SFO}$

$========$ CHANNEL f2 ========

CPDPRG [2 waltz16

NUC2 $1 \mathrm{H}$

PCPD2 80.00 usec

PL12 $11.00 \mathrm{~dB}$

PL13 $13.69 \mathrm{~dB}$

PL2W $10.56200695 \mathrm{~W}$

PL12W $\quad 0.35871249 \mathrm{~W}$

PL13W $0.29767781 W$

SF02 $400.1316005 \mathrm{MHZ}$

F2 - Processing parameters

SI 32768

SF $100.6127484 \mathrm{MHz}$

WDW

$\begin{array}{lll}\text { SSB } & 0 & 1.00 \mathrm{~Hz}\end{array}$

$\begin{array}{lll}\mathrm{GB} & 0 & 1.40\end{array}$

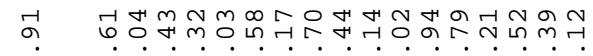

它

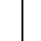

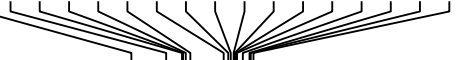

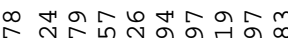

ón.

(1)

คั

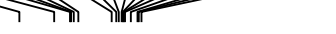

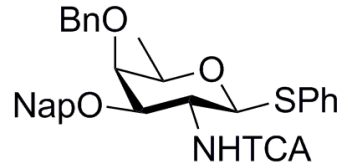

NHTCA

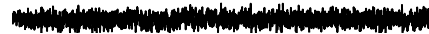

$230 \quad 220-210 \quad 200$

$190 \quad 180$

$\begin{array}{lll}80 & 170 \quad 160\end{array}$

$\begin{array}{lll}40 & 130 & 120\end{array}$

110 


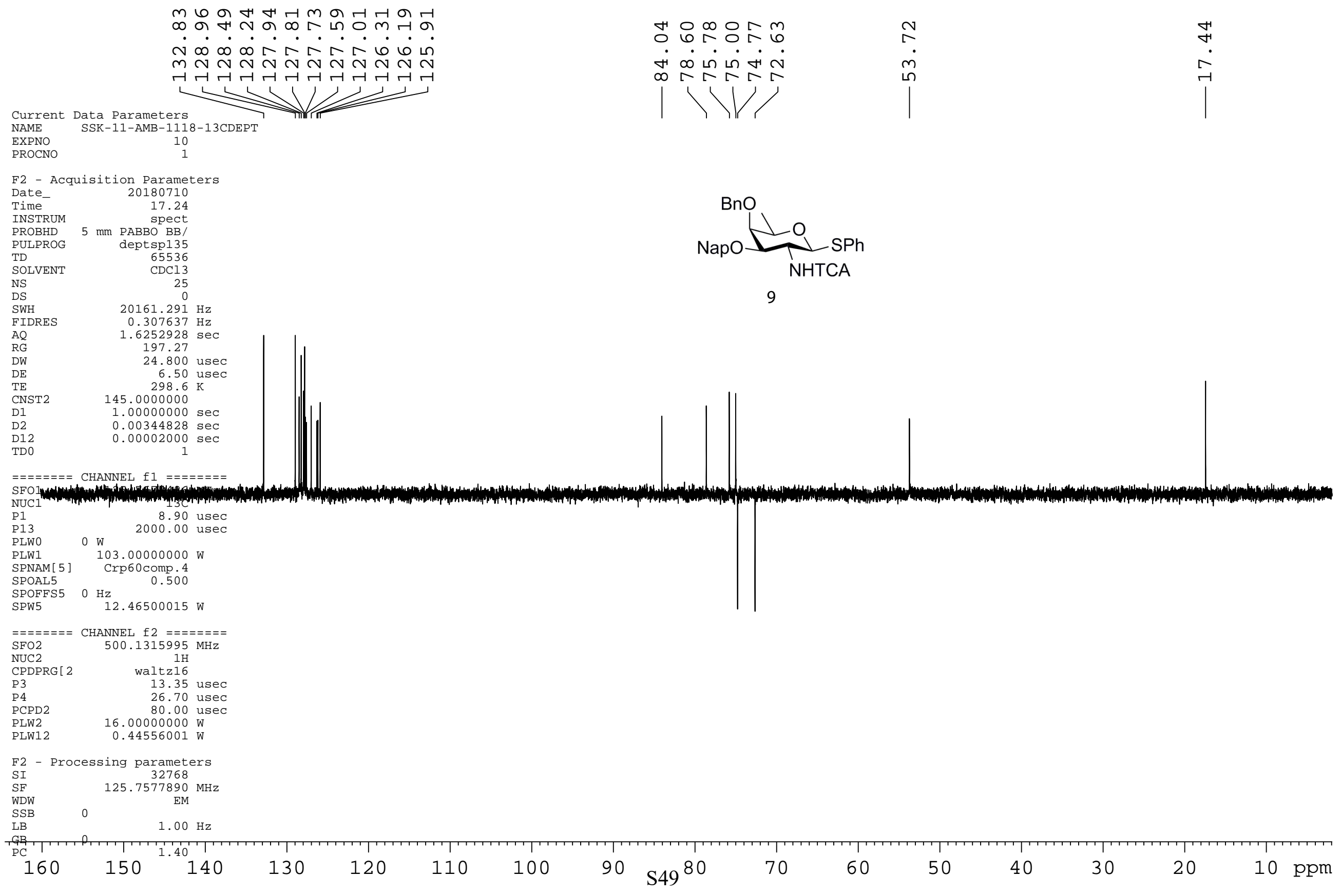




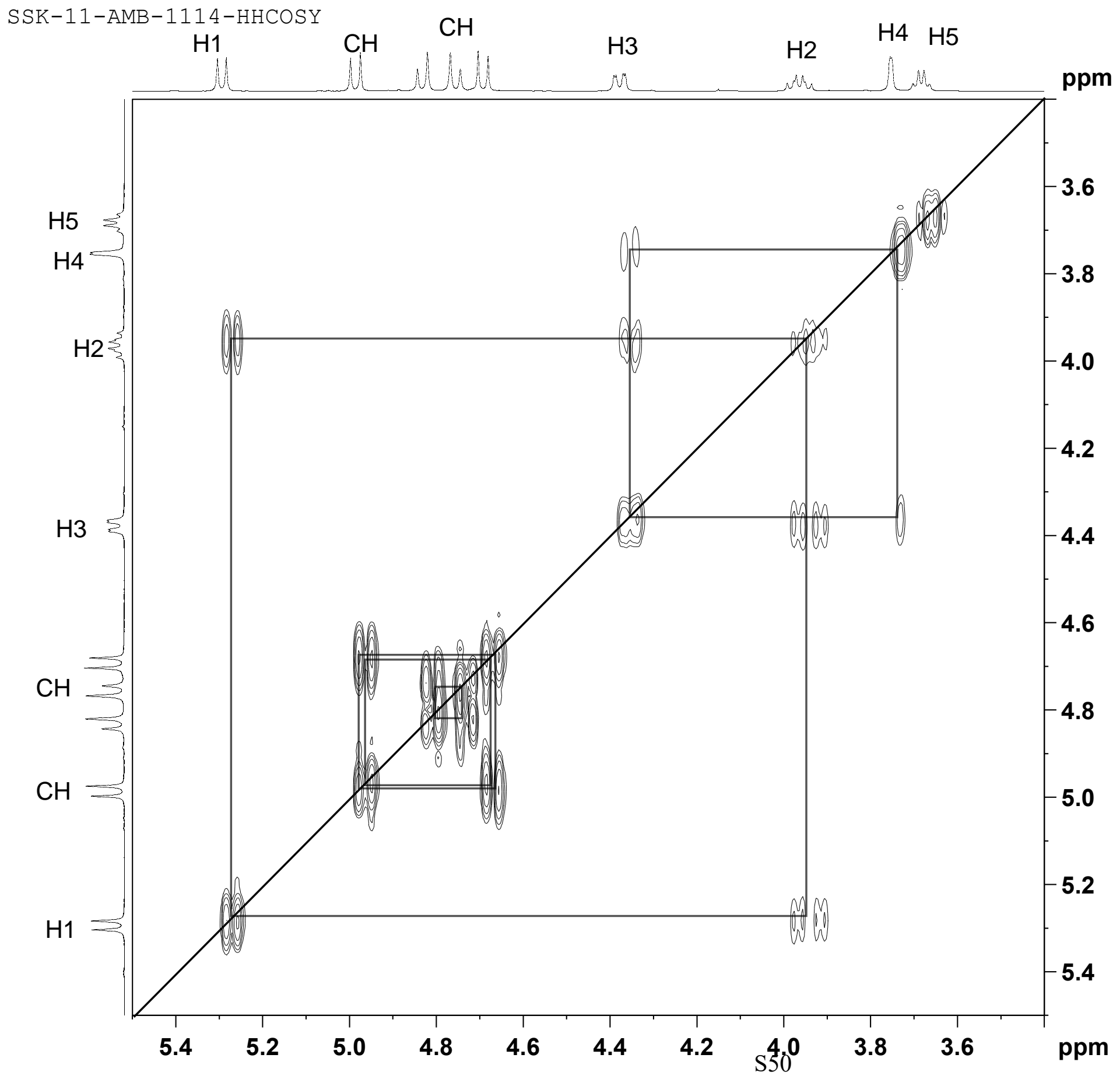


Current Data Parameters

NAME SSK-11-AMB-1186-1H 1

F2 - Acquisition Parameters

Date_ 20181102

\begin{tabular}{lr} 
Time & 21.17 \\
\hline
\end{tabular}

spect
PROBHD $5 \mathrm{~mm}$ PABBO BB/

PULPROG

$\begin{array}{ll}\text { TD } & 65536 \\ \text { SOLVENT } & \text { CDCl3 }\end{array}$

\begin{tabular}{lr}
$N S$ & CDC13 \\
DS & 11 \\
\hline
\end{tabular}

SWH $\quad 10000.000 \mathrm{~Hz}$

FIDRES $\quad 3.152588 \mathrm{~Hz}$

$\begin{array}{lr}\text { AQ } & 3.2767999 \\ \text { RG } & 30.72\end{array}$

DW $\quad 50.000$ usec

$\begin{array}{lr}\text { DE } & 6.50 \text { usec }\end{array}$

D1 $1.0000000 \% \mathrm{sec}$

TD॰ 1

$========$ CHANNEL $\mathrm{f} 1=======$ SF01 $500.1330885 \mathrm{MHZ}$

NUC1 $1 \mathrm{H}$

$\begin{array}{lr}\text { P1 } & 13.35 \text { usec } \\ \text { PLW1 } & 16.00000000 \mathrm{~W}\end{array}$

F2 - Processing parameters

SI 65536

SF $\quad 500.1300000 \mathrm{MHz}$

WDW $\quad \odot \quad$ EM

$\begin{array}{lll}\text { LB } & 0.30 \mathrm{~Hz}\end{array}$

$\begin{array}{lll}\mathrm{GB} & \odot & 1.0 \odot\end{array}$
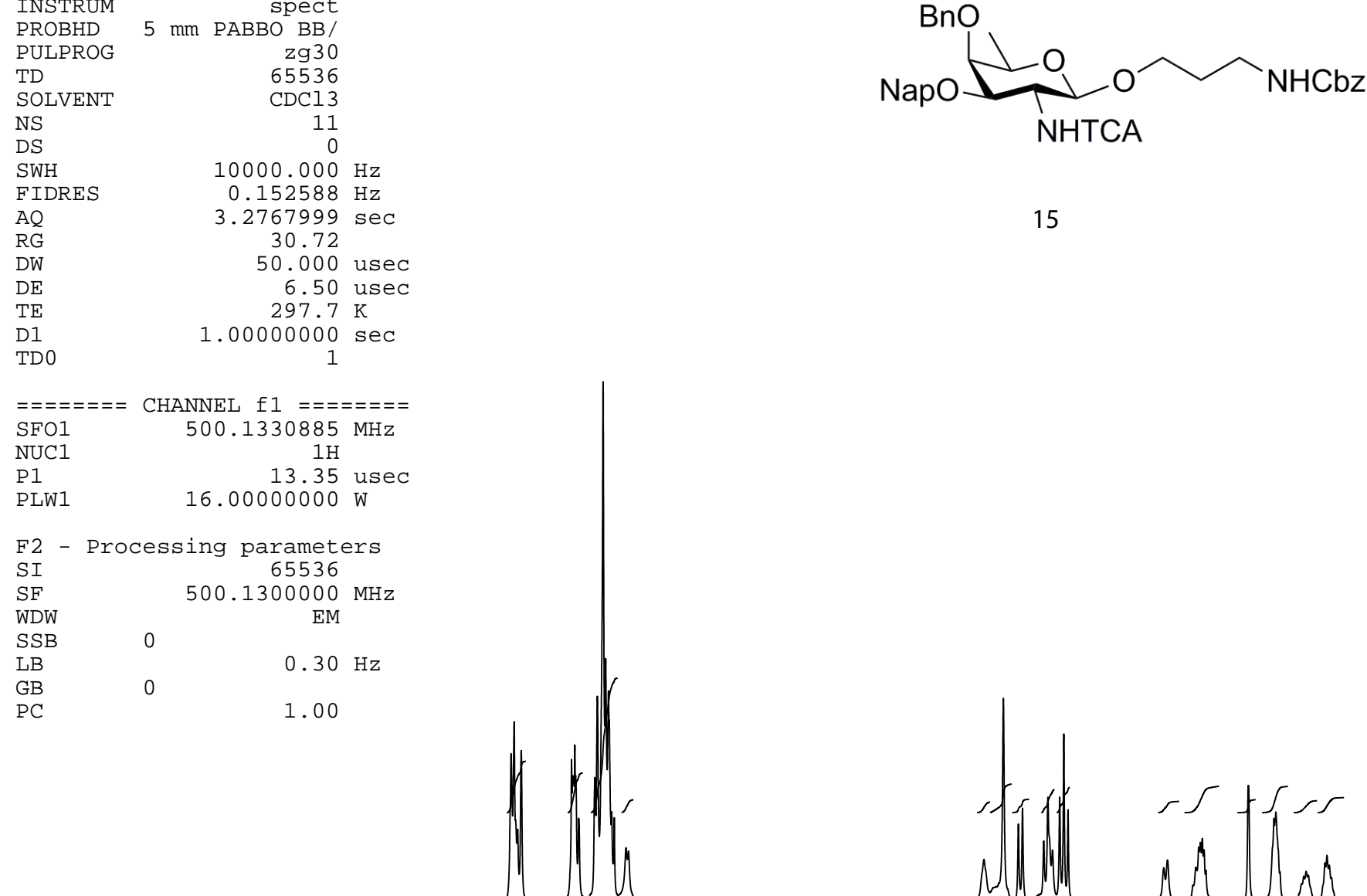

15

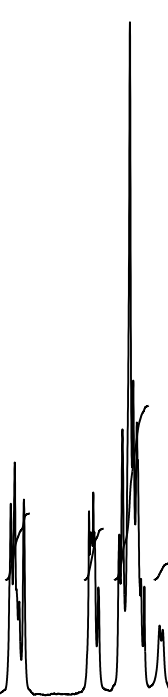

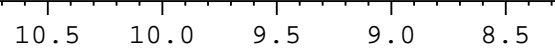
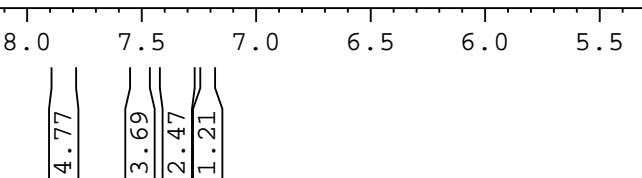

$5.0 \quad 4.5$
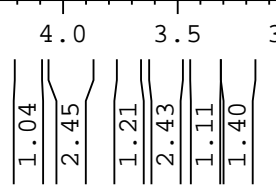

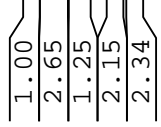

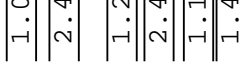

3.0

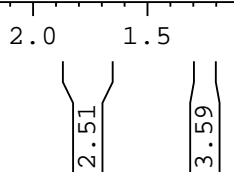

$1.0 \quad 0.5 \quad 0.0 \quad \mathrm{ppm}$ 
m

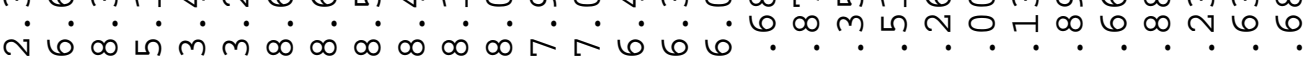

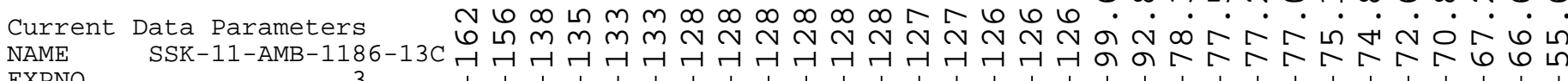

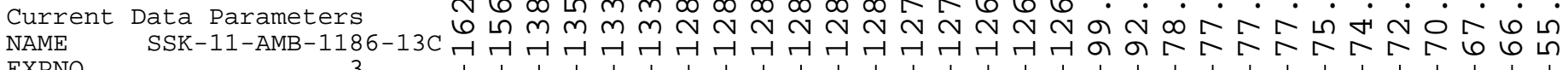

EXPNO 1

F2 - Acquisition Parameters

Date_ 20181102

Time

21.21
spect

PROBHD

$5 \mathrm{~mm}$ PABBO BB/

PULPROG

zgpg30
65536

$\begin{array}{ll}\text { SOLVENT } & 65536 \\ & \mathrm{CDCl3}\end{array}$

NS

DS

110

SWH 29761.904 Hz

FIDRES $\quad 0.454131 \mathrm{~Hz}$

AQ $\quad 1.1010048 \mathrm{sec}$

RG $\quad 197.27$

$\begin{array}{lr}\text { DW } & 16.800 \text { usec } \\ \text { DE } & 6.50 \text { usec }\end{array}$

TE $298.3 \mathrm{~K}$

D1 $1.00000 \odot \odot \odot \mathrm{sec}$

$\begin{array}{ll}\text { D11 } & 0.03000000\end{array}$

$========$ CHANNEL f1 ======== SF01 125.7703637 MHZ NUC1 $13 \mathrm{C}$

P1 8.90 usec

PLW1 $103.00000000 \mathrm{~W}$

$========$ CHANNEL $f 2$ ========

SF02 500.1320005 MHz

NUC2 $1 \mathrm{H}$

CPDPRG [2 waltz16

$\begin{array}{lr}\text { PCPD2 } & 80.00 \text { used }\end{array}$

PLW2 16.00000000 W

PLW12 $\quad 0.44556001 \mathrm{~W}$

F2 - Processing parameters

$\begin{array}{lr}\text { SI } & 32768 \\ \text { SF } & 125.7577716 \mathrm{MHz}\end{array}$

WDW

$\begin{array}{lll}\text { SSB } & 0 & 1.00 \mathrm{~Hz}\end{array}$

$\mathrm{GB} \quad \odot \quad 1.40$

PC 1.40 
SSK - 11-AMB - $1186-1 \mathrm{H}$

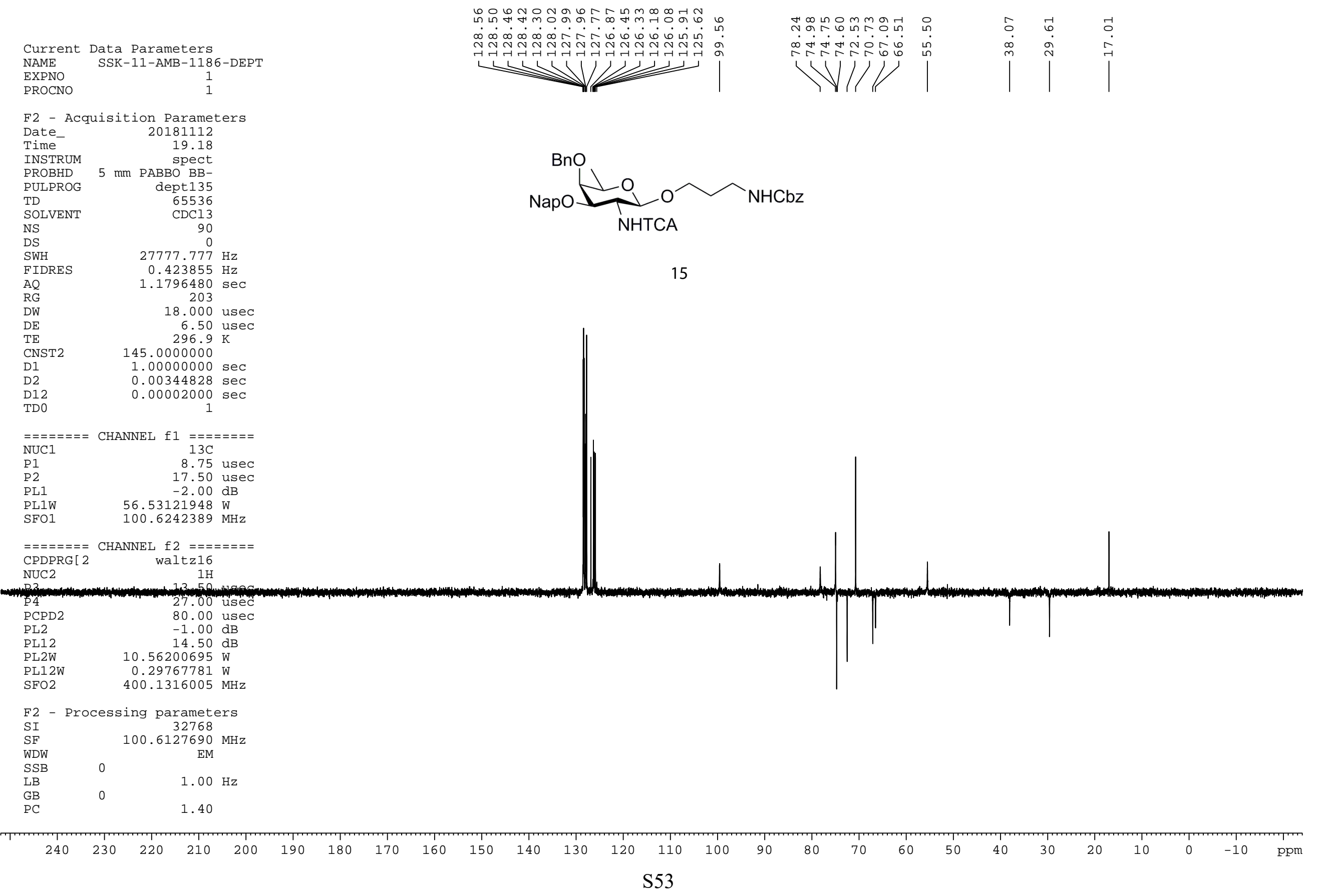



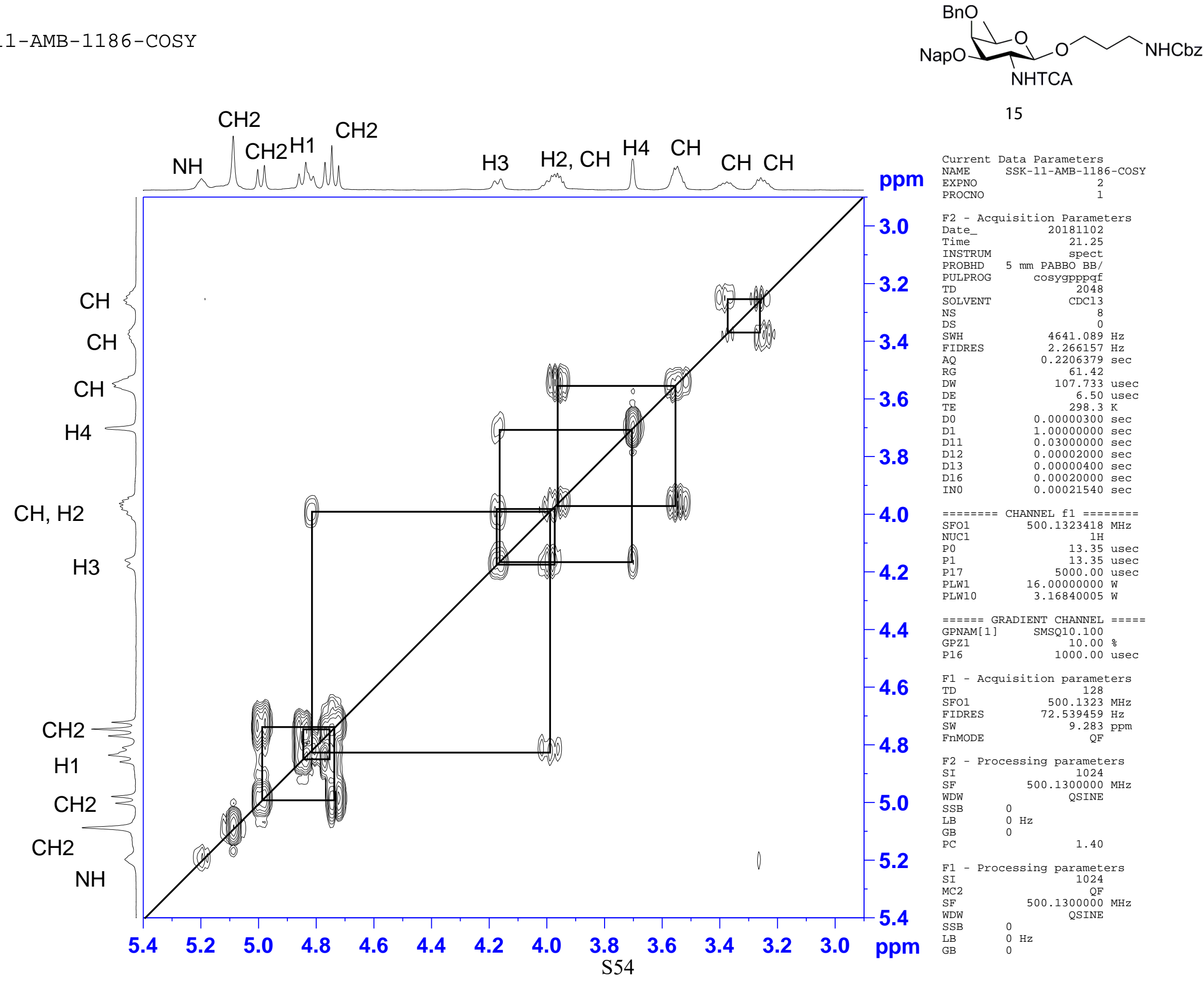


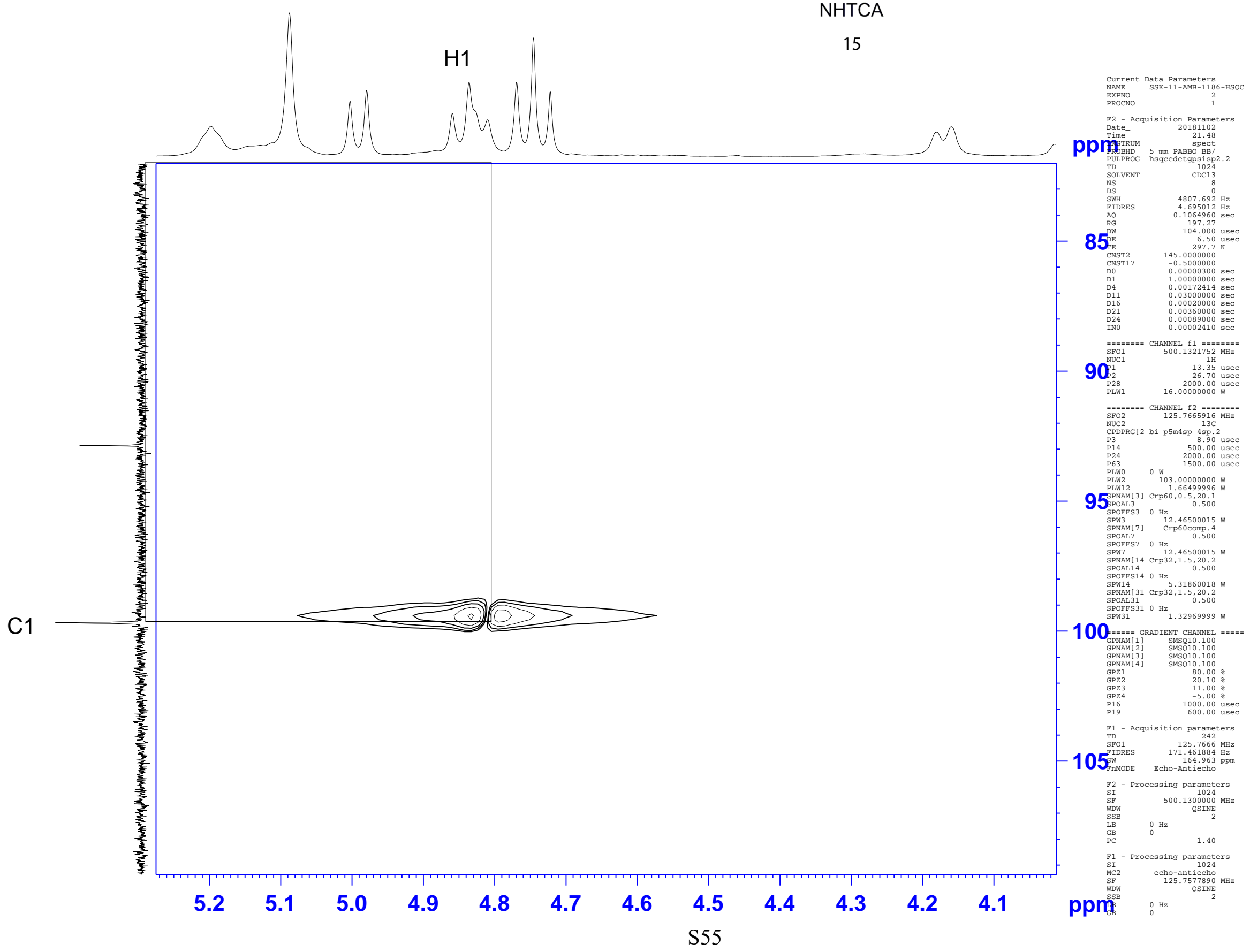


SSK-11-AMB-1132-1H

Current Data Parameters

NAME SSK-11-AMB-1132-1H

$\begin{array}{lr}\text { EXPNO } & 10 \\ \text { PROCNO } & 1\end{array}$

F2 - Acquisition Parameters

Date_ 20180803

Time 7.41

$5 \mathrm{~mm}$ PABBO

PULPROG 5 TIm PABBO BB-

zg30

SOLVENT

CDC13

NS

DSH

SWH

$A Q$

RG

DW

DE

TE

D1 0

$8223.685 \mathrm{~Hz}$

$3.2998593 \mathrm{sec}$

57

60.800 usec

6.50 usec

$1.00000000 \mathrm{sec}$
==ニ==
NUC1
P1
PL1
PL1W $-1.00 \mathrm{~dB}$

1

SF01

$40 \odot .1324710 \mathrm{MHz}$

F2 - Processing parameters

SI 32768

SF $400.1300095 \mathrm{MHz}$

WDW

SSB

LB

$\odot$

$\odot .30 \mathrm{~Hz}$

$\mathrm{PC}$

1.00
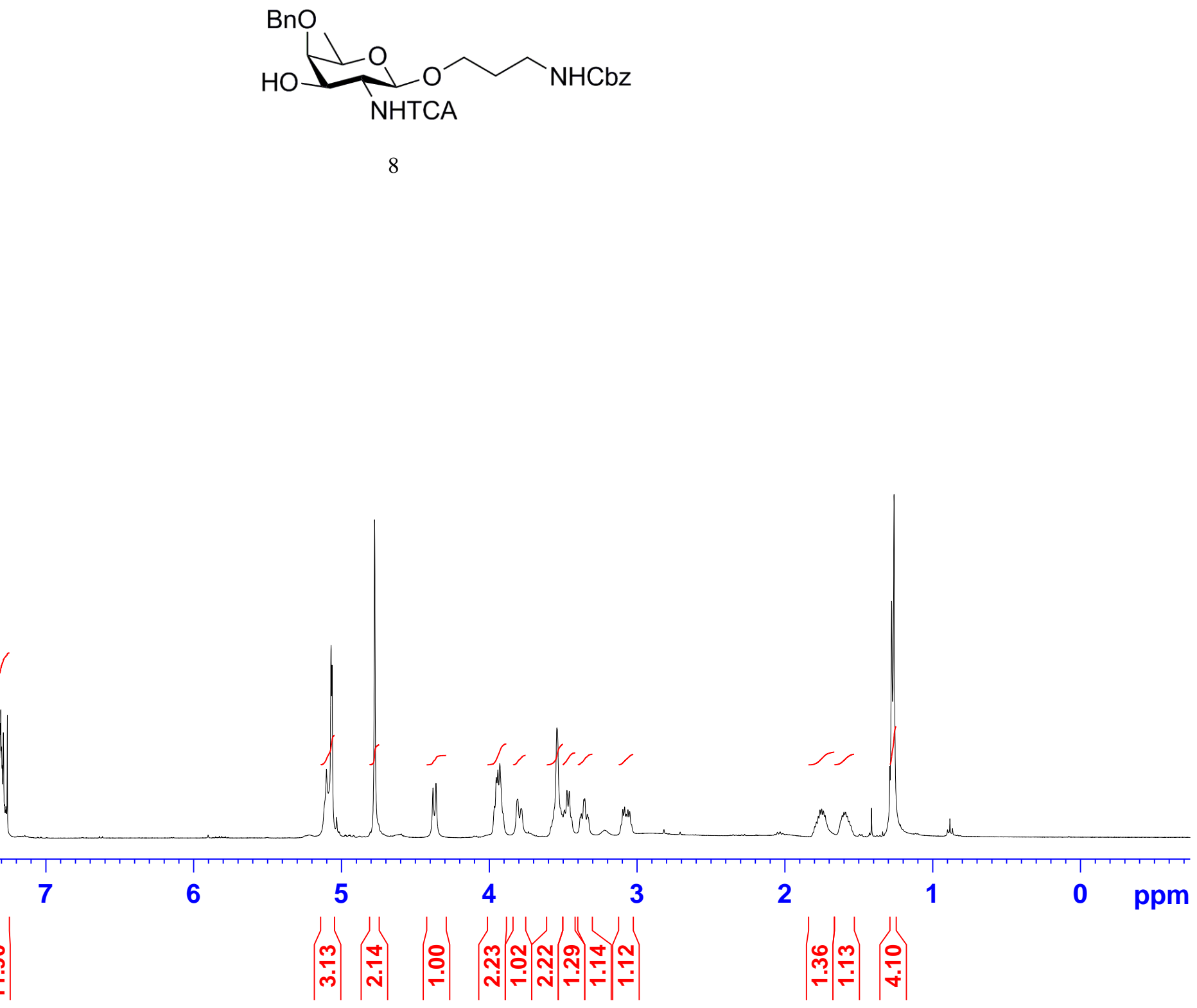
SSK - 11 - AMB - $1132-13 C$

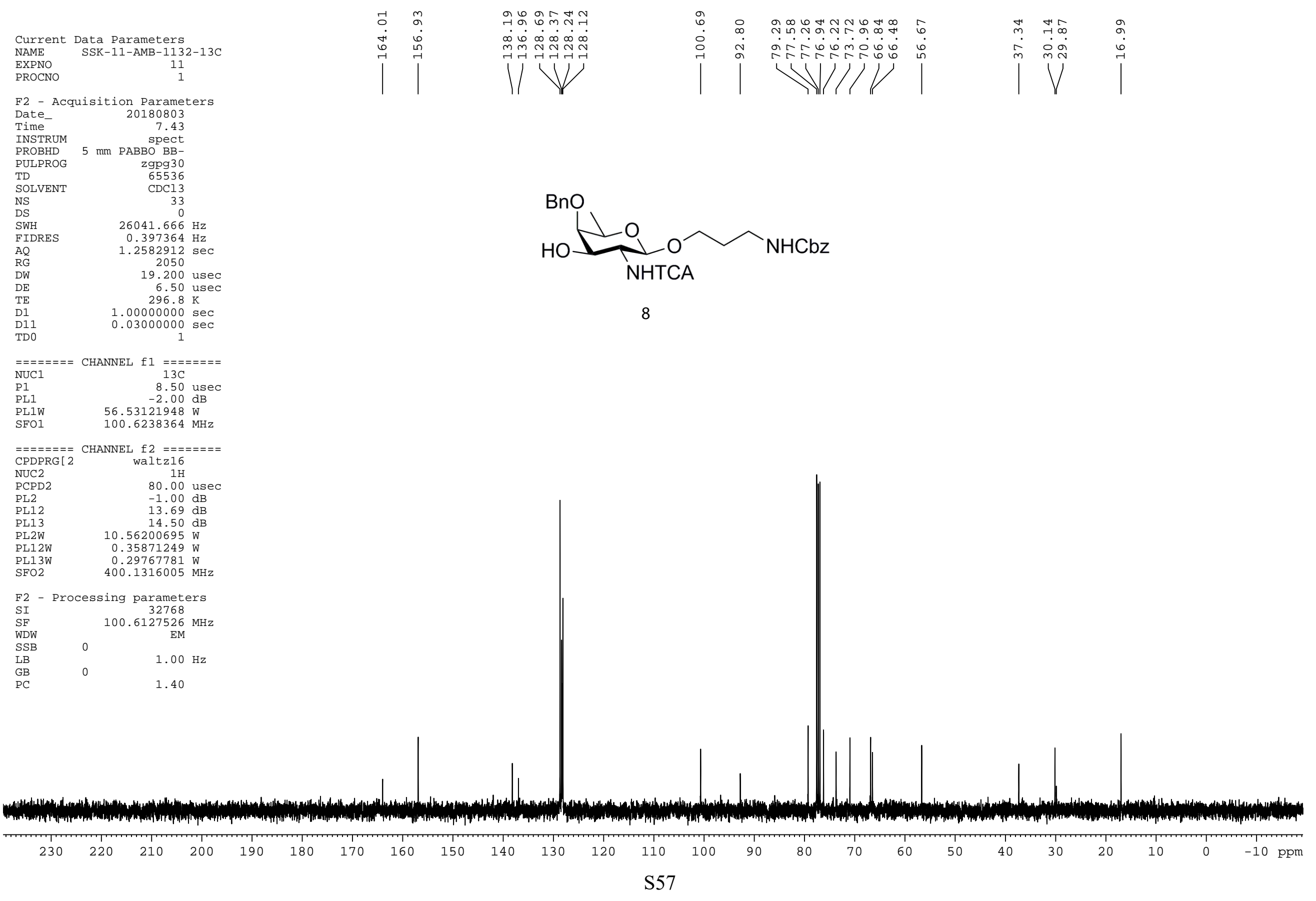




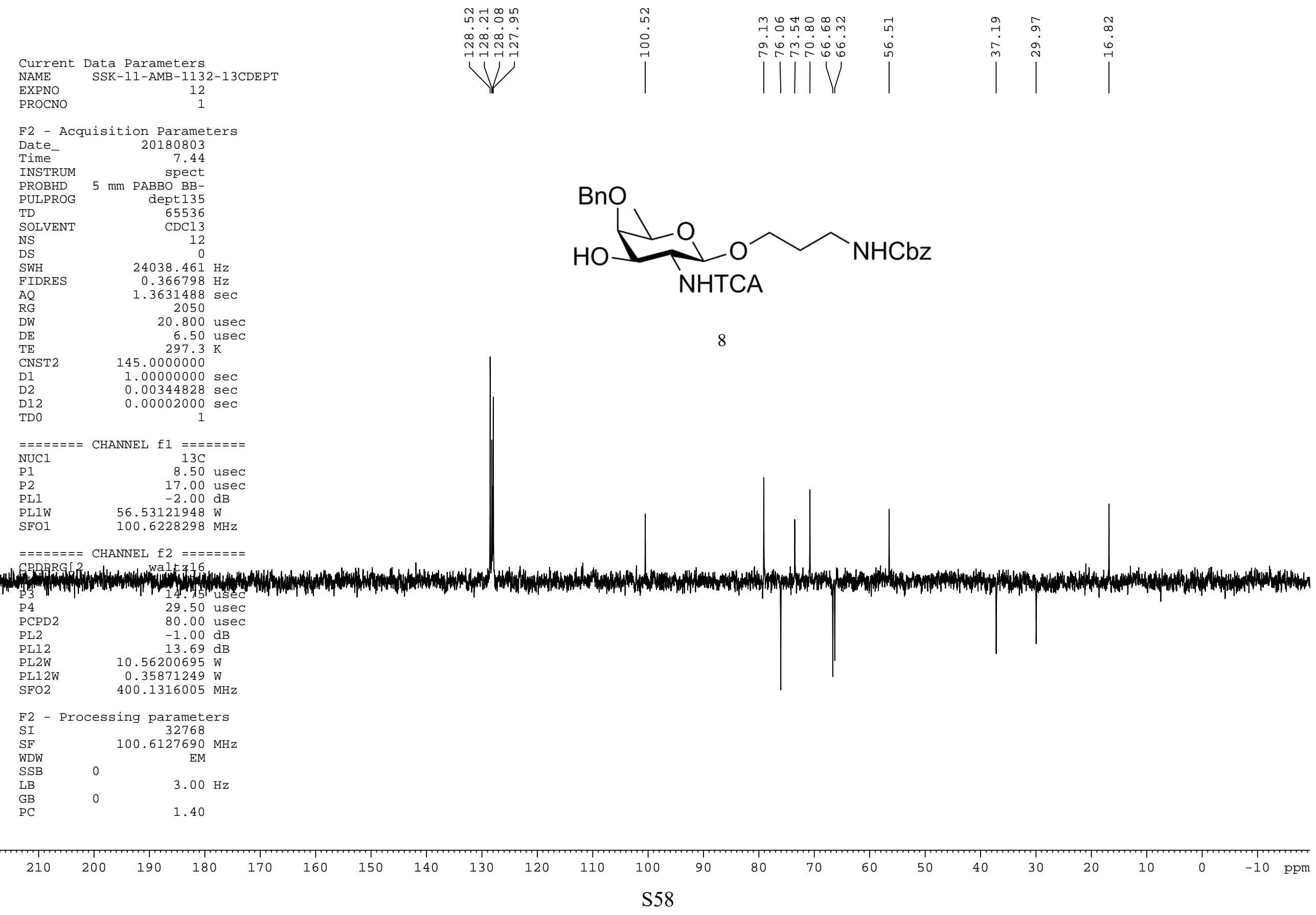


SSK-11-AMB-1132-HHCOSY

$\mathrm{NH}, \mathrm{CH} 2$ Of $\mathrm{Cbz}$ $\mathrm{CH} 2$ Of $\mathrm{Bn}$

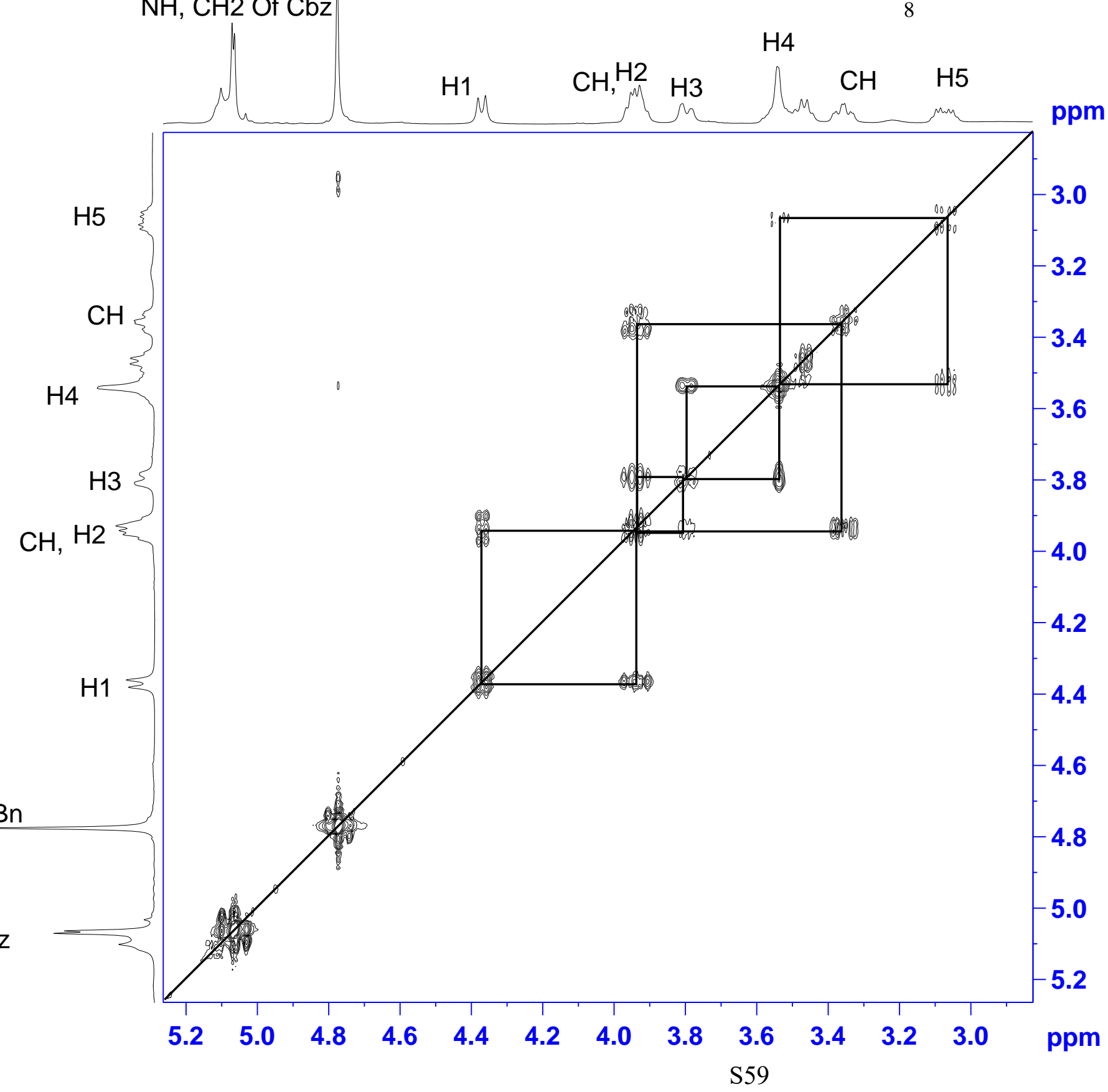

Current Data Parameters

列 EXPNO

F2 - Acquisition Parameters

Date

spect
PROBHD $5 \mathrm{~mm}$ PABBO BB-

PULPROG COsygpqf

TD
SOLVENT

NS

DS

SWH
FIDRES
AQ

$\mathrm{AQ}$
$\mathrm{RG}$
$\mathrm{DW}$

DW

TE

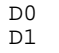

D1
D13
D16 $\mathrm{CDCl} 3$
4 1764.291 Hz $0.5804032 \mathrm{sz}$ 283.400 usec 6.50 use $0.00000300 \mathrm{sec}$ $1.00000000 \mathrm{sec}$ $0.00000400 \mathrm{sec}$ $\odot .00020000 \mathrm{sec}$

$=======$ CHANNEL $\mathrm{f} 1 \mathrm{l}=======$
NUC1 $\begin{array}{ll}\text { NUC1 } & 1 \mathrm{H} \\ \text { P0 } & 14.75 \mathrm{usec} \\ \text { P1 } & 14.75 \mathrm{usec}\end{array}$ PL1 $\quad-1.00 \mathrm{~dB}$ $\begin{array}{ll}\text { PL1W } & 10.56200695 \mathrm{~W} \\ \text { SF01 } & 400.1312984 \mathrm{MHz}\end{array}$ $======$ GRADIENT CHANNEL $=====$ $======$ GRADIENT SINANEL $\begin{array}{lr}\text { GPZ1 } & 10.00 \% \\ \text { P16 } & 1000.00 \%\end{array}$

F1 - Acquisition parameters

$\begin{array}{lr}\text { SF01 } & 400.1313 \mathrm{MHz} \\ \text { FIDRES } & 21.647840 \mathrm{~Hz}\end{array}$ $\begin{array}{lr}\text { FIDRES } & 21.647840 \mathrm{~Hz} \\ \text { SW } & 4.409 \mathrm{ppm}\end{array}$

F2 - Processing parameters

SI 2048

SF $400.1300095 \mathrm{MHZ}$

$\begin{array}{ll}\text { WDW } & \\ \text { SSB } & \odot \\ \text { LB } & \odot \mathrm{Hz}\end{array}$

GB $\quad \odot$

PC 1.40

F1 - Processing parameters

$\begin{array}{lc}\text { SI } & 512 \\ \text { MC2 } & \text { QF } \\ \text { SF } & 400.1300095 \mathrm{MHz}\end{array}$

$\begin{array}{ll}\text { WDW } & \\ \text { SSB } & 0 \\ \text { LB } & 0\end{array}$

$\mathrm{CH} 2 \mathrm{Of} \mathrm{Cbz}$

$\mathrm{NH}$ 


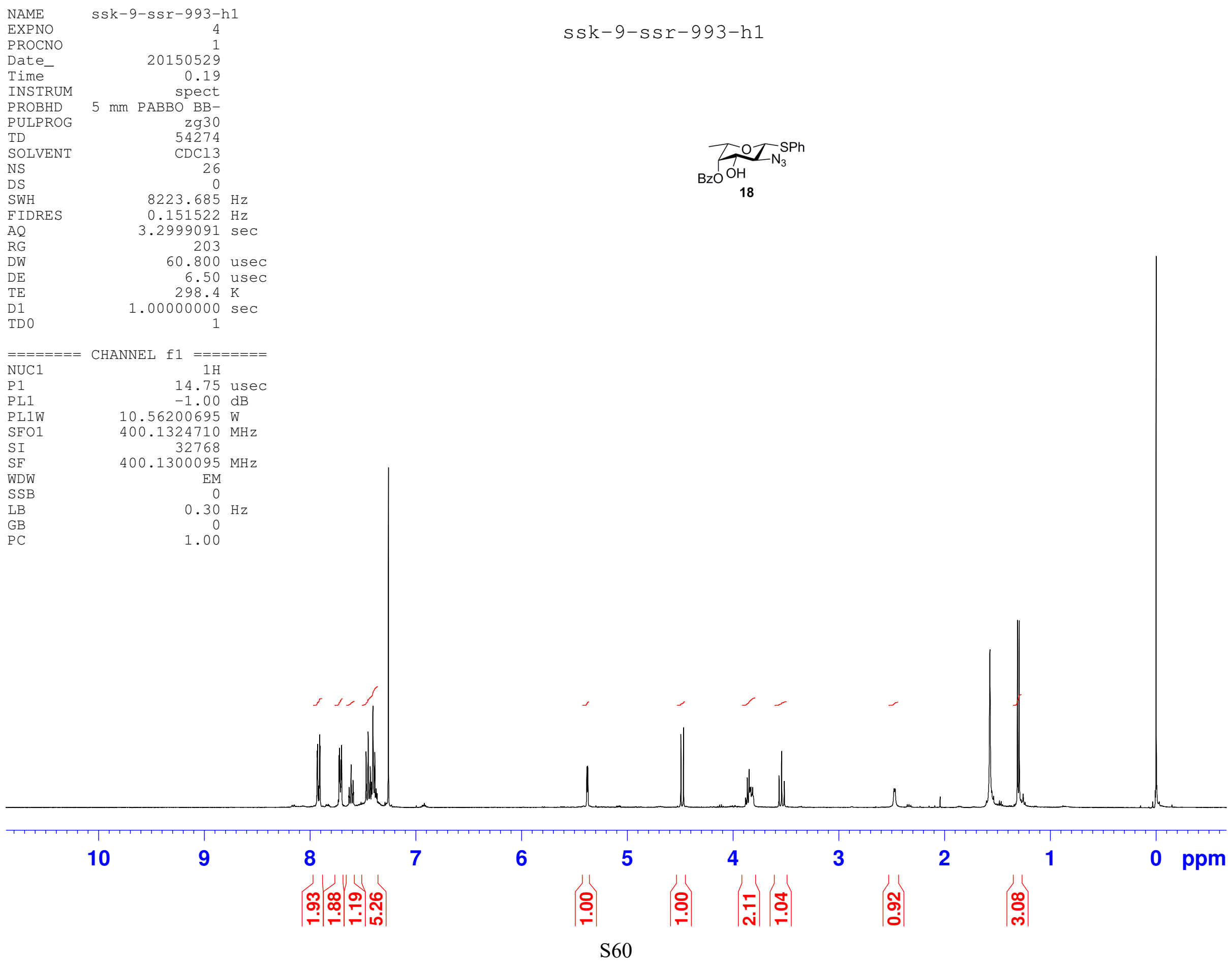




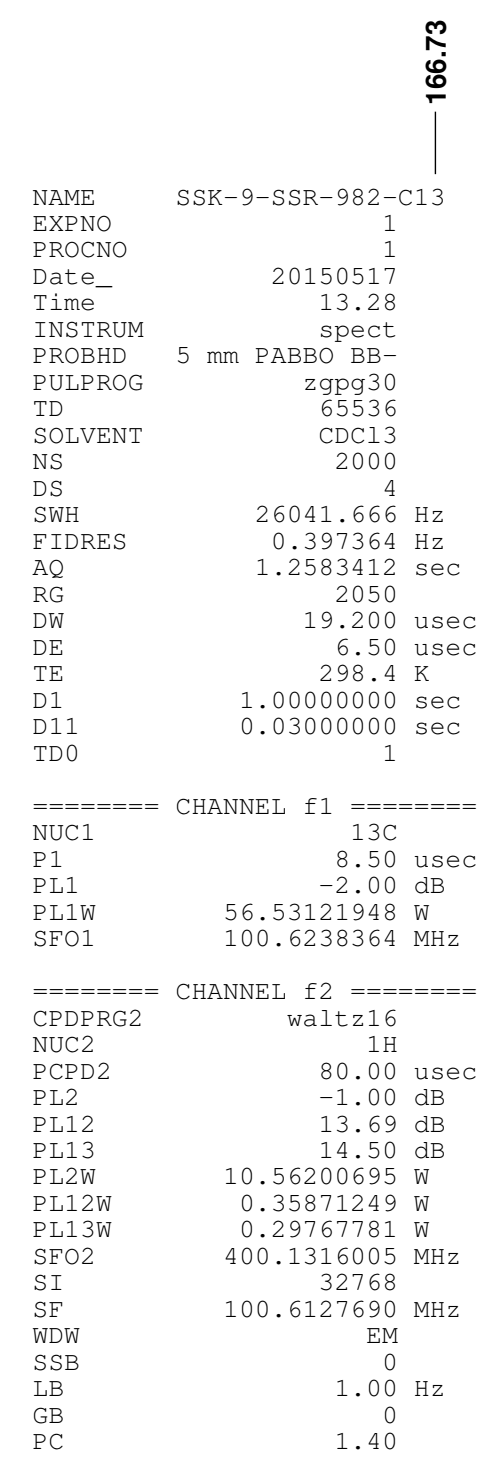

PROCNO

Time

IDRES

$\mathrm{DE}$

D1

PLI

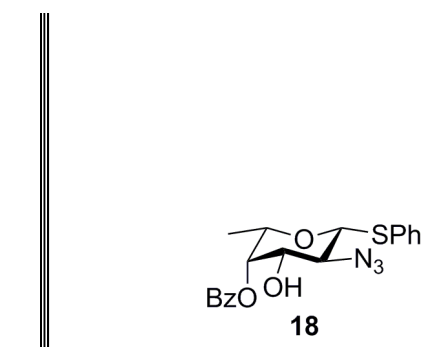

18 


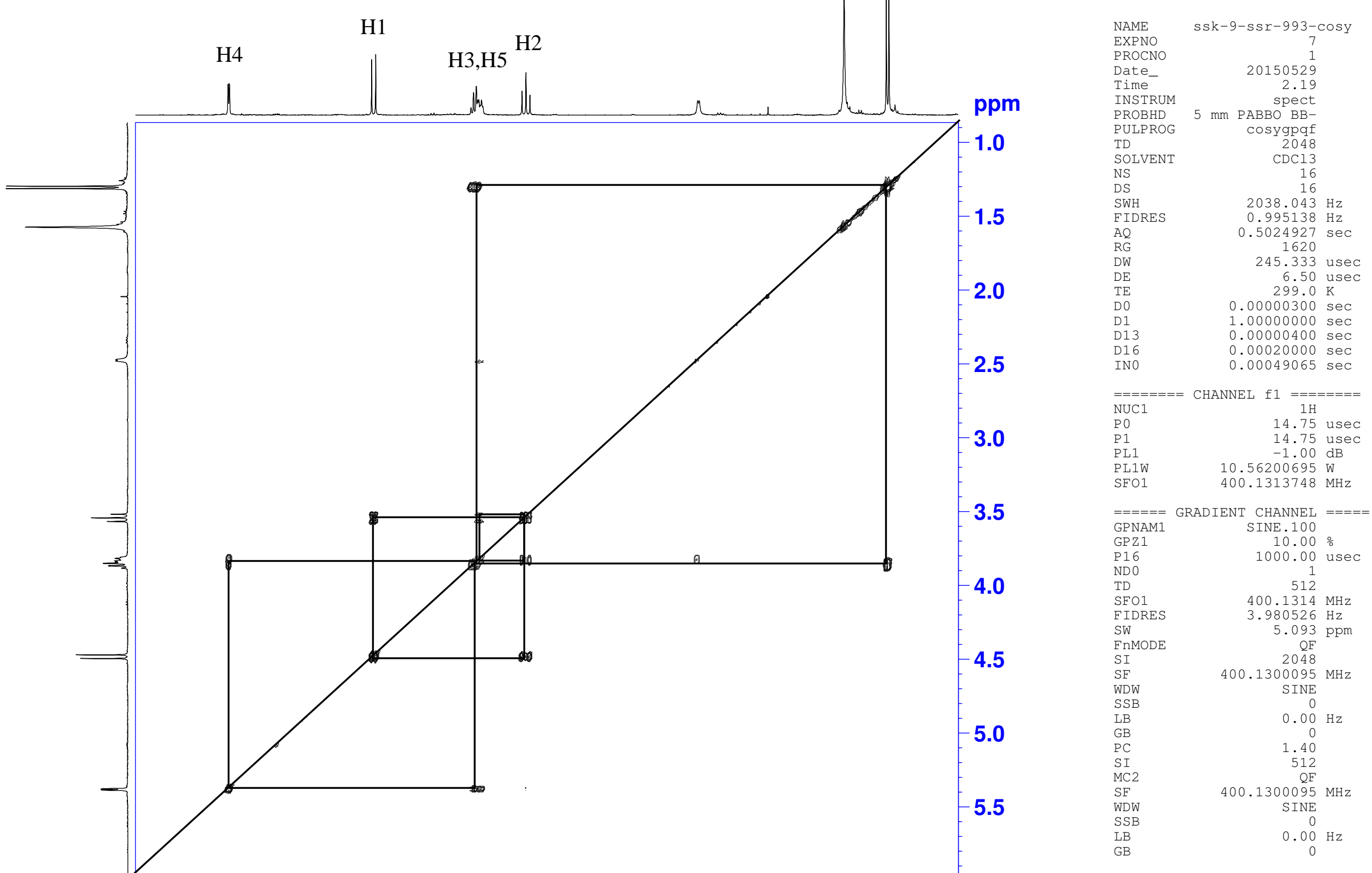




\section{SSK - 11-AMB - 919-1H}

Current Data Parameters

NAME SSK-11-AMB-919-1H

EXPNO

1

F2 - Acquisition Parameters

Date_ 20170929

$\begin{array}{lr}\text { Time- } & 15.10 \\ \text { INSTRUM } & \end{array}$

INSTRUM
PROBHD $5 \mathrm{~mm} \mathrm{PABBO}$ BB-

PROBHD $5 \mathrm{~mm}$ PABBO BB-
PULPROG

$\begin{array}{lr}\text { TD } & \text { Zg3 } \\ & 54274\end{array}$

SOLVENT CDCl3

NS

$\begin{array}{lr}\text { DS } & 0 \\ \text { SWH } & 8223.685 \mathrm{~Hz}\end{array}$

FIDRES $\quad 0.151522 \mathrm{~Hz}$

$\begin{array}{lr}\text { AQ } & 3.2998593 \mathrm{sec} \\ \mathrm{RG} & 57\end{array}$

$\begin{array}{lr}\mathrm{DW} & 60.800 \text { usec } \\ \mathrm{DE} & 6.50 \text { usec }\end{array}$

TE $\quad 6.50$ usec

D1 $\quad 1.00000000 \mathrm{sec}$

TDO

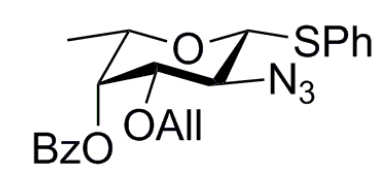

$7 a$

$=======$ CHANNEL $\mathrm{f} 1$ ======

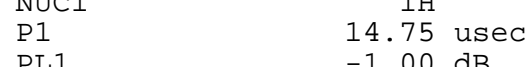

$\begin{array}{ll}\text { PL1 } & -1.00 \mathrm{~dB} \\ \text { PL1W } & 10.56200695 \mathrm{~W}\end{array}$

SF01 $400.1324710 \mathrm{MHz}$

F2 - Processing parameters

SI - Processing parameters

$\begin{array}{lr}\text { SI } & 32768 \\ \text { SF } & 400.1300099 \mathrm{MHz}\end{array}$

$\begin{array}{lr}\text { SF } & 400.1300099 \\ \text { WDW } & \text { EM }\end{array}$

SSB $\quad \odot$

$\begin{array}{ll}\text { LB } & 0.30 \mathrm{~Hz}\end{array}$

$\mathrm{PC}$

1.00

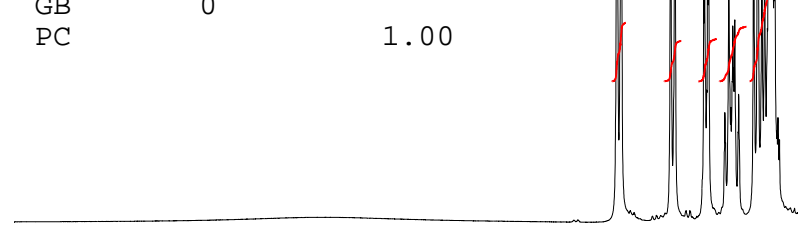

10

9

8

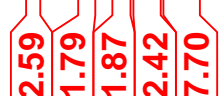

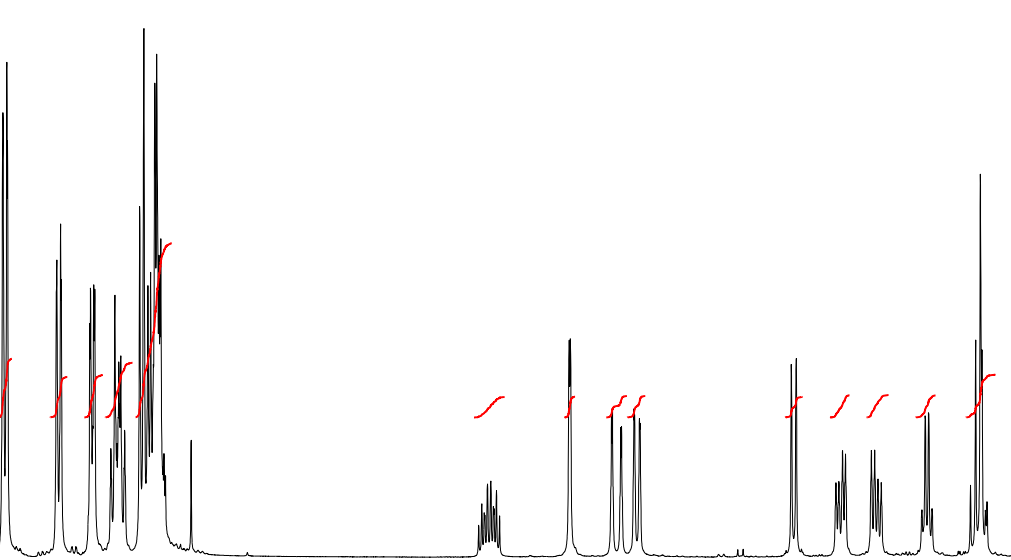

5

4

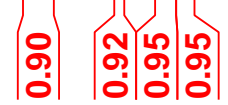

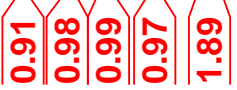

3

2

1

일 
SSK-11-AMB - 919-13C

Current Data Parameters

$\begin{array}{lc}\text { NAME } & \text { SSK-11-AMB-919-13C } \\ \text { EXPNO } & 2 \\ \text { PROCNO } & 1\end{array}$

EXPNO

2 - Acquisition Parameters

Date_ 20170929

Time $\quad 15.13$

INSTRUM Spect

PROBHD $5 \mathrm{~mm}$ PABBO BB-

PULPROG $\quad$ zgpg30

TD

zgpg 30
65536

SOLVENT

$\mathrm{CDCl}$
73

DS

$26041.666 \mathrm{~Hz}$

$\begin{array}{lrl}\text { FIDRES } & 0.397364 \mathrm{~Hz} \\ \text { AQ } & 1.2582912 \mathrm{sec} \\ \text { RG } & 2050 \\ \text { DW } & 19.200 \text { usec }\end{array}$

$\begin{array}{lrl}\text { FIDRES } & 0.397364 \mathrm{~Hz} \\ \text { AQ } & 1.2582912 \mathrm{sec} \\ \text { RG } & 2050 \\ \text { DW } & 19.200 \text { usec }\end{array}$

$\begin{array}{lrl}\text { FIDRES } & 0.397364 \mathrm{~Hz} \\ \text { AQ } & 1.2582912 \mathrm{sec} \\ \text { RG } & 2050 \\ \text { DW } & 19.200 \text { usec }\end{array}$ 6.50 usec

D11

1

\section{$=======$ CHANNEL $\mathrm{f} 1$}

NUC1

$$
13 \mathrm{C}
$$

$13 \mathrm{C}$

P1

$2.00 \mathrm{~dB}$

PL1W 56.53121948

$========$ CHANNEL f2 ========

CPDPRG [2 waltz16

$\begin{array}{lr}\text { NUC2 } & 1 \mathrm{H} \\ \text { PCPD2 } & 80.00 \text { usec }\end{array}$

$\begin{array}{ll}8 \odot . \odot \odot & \text { us } \\ -1.0 \odot \mathrm{dB} & -13.09\end{array}$

$13.69 \mathrm{~dB}$

PL13 $14.50 \mathrm{~dB}$

$\begin{array}{lr}\text { PL2W } & 10.56200695 \\ \text { PL12W } & 0.35871249 \mathrm{~W}\end{array}$

PL13W $\quad 0.29767781 \mathrm{~W}$

SF02 400.1316005 MHz

F2 - Processing parameters

SI 32768

$\begin{array}{lr}\text { SF } & 100.6127509 \mathrm{MHz} \\ \text { WDW } & \text { EM }\end{array}$

$\begin{array}{lll}\text { SSB } & 0 & 1.00 \mathrm{~Hz}\end{array}$

$\begin{array}{lll}\mathrm{LB} & 0 & 1.00 \\ \mathrm{~GB} & 0 & 1.40\end{array}$

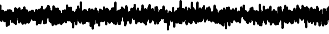




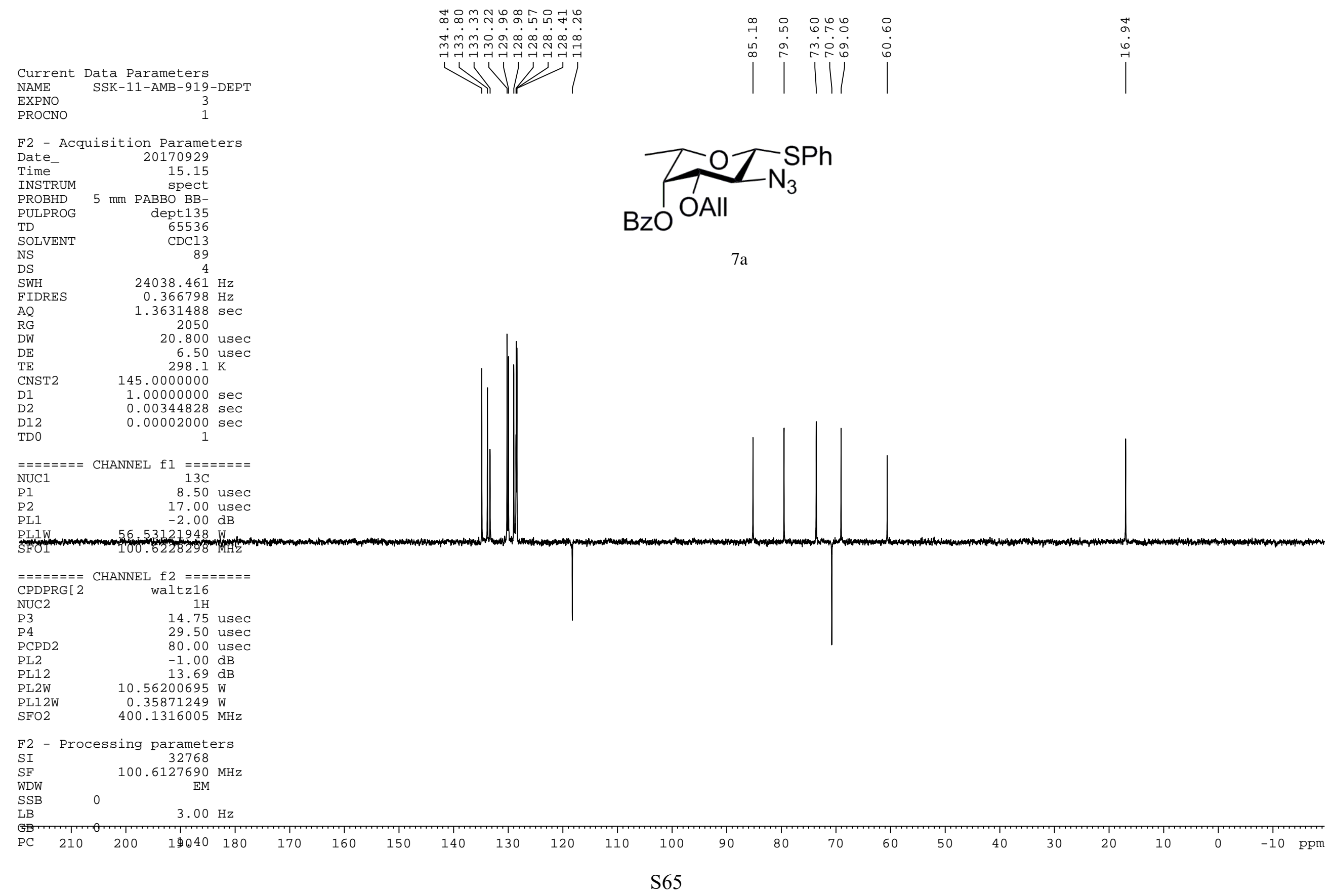


SSK-11-AMB - 919-COSY

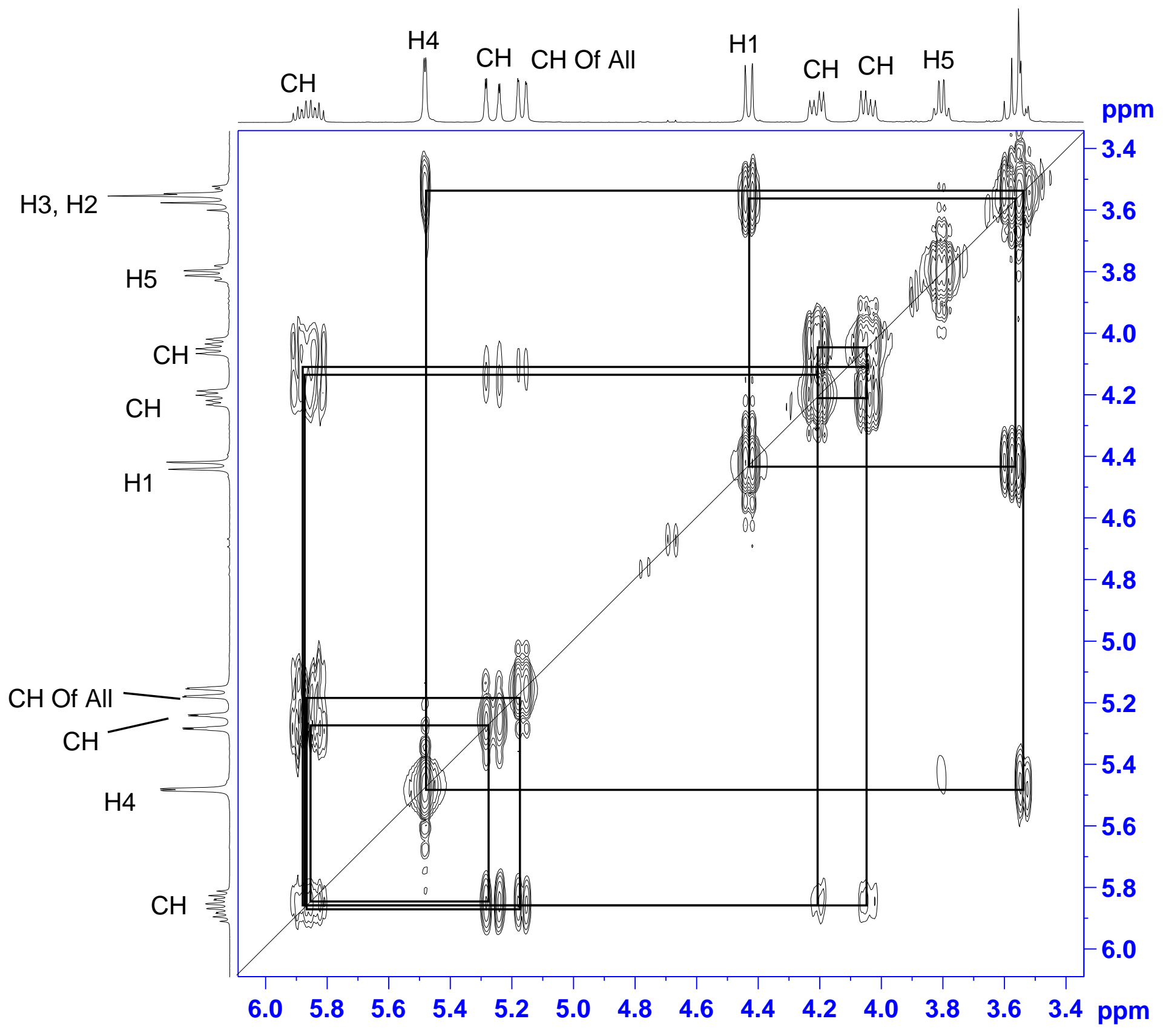

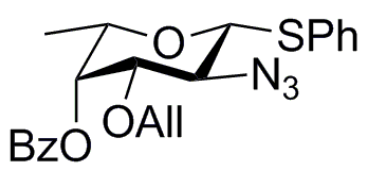

$7 a$

Current Data Parameters
NAME SSK-11-AMB-919-COSY EXPNO

F2 - Acquisition Parameters $\begin{array}{lr}\text { Date } & 20170929 \\ \text { Time } & 15.19\end{array}$

spect
INSTRUM
PROBHD $5 \mathrm{~mm}$ PABBO BB-

PROBHD
PULPROG $\mathrm{mm}$ PABBO BB-
cosygpqf

$\begin{array}{lr}\text { PULPOG } & \text { COSYgpqf } \\ \text { TD } & 2048 \\ \text { SOLVENT } & \text { CDC13 } \\ \text { NS } & 16\end{array}$

$\begin{array}{lc}\text { DS } & 16 \\ \text { SWH } & 3506.311 \mathrm{~Hz} \\ \text { FIDRES } & 1.712066 \mathrm{~Hz}\end{array}$

$\begin{array}{lr}\text { FIDRES } & 1.712066 \mathrm{~Hz} \\ \mathrm{AQ} & 0.2920448 \mathrm{sec}\end{array}$

$\begin{array}{lr}\text { AQ } & 1.2920448 \mathrm{sec} \\ \text { RG } & 2.56 \\ \text { DW } & 142.600 \text { usec } \\ \text { DE } & 6.50 \text { us }\end{array}$

DE $\quad 6.50$ use

TE $\quad 0.00000300 \mathrm{~K}$

$\begin{array}{ll}\text { D1 } & 1.000000000 \mathrm{sec} \\ \text { D13 } & 0.00000400 \mathrm{sec}\end{array}$

D16 $\quad 0.00020000 \mathrm{sec}$

$=======$ CHANNEL $f 1$ =======

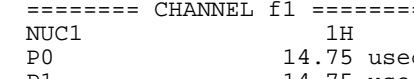

$\begin{array}{ll}\text { PO } & 14.75 \text { usec } \\ \text { P1 } & 14.75 \text { usec }\end{array}$

PL1W $10.56200695 \mathrm{~W}$

SF01 400.1318766 MHZ

$======$ GRADIENT CHANNEL $=====$

$======$ GRADIENT CHANNEL
GPNAM[1] SINE.100

$\begin{array}{lc}\text { GPZ1 } & 10.00 \% \\ \text { P16 } & 1000.00 \text { usec }\end{array}$

F1 - Acquisition parameters

TD - Acquisition parameters

$\begin{array}{lr}\text { SF01 } & 400.1319 \mathrm{MHz} \\ \text { FIDRES } & 98.769455 \mathrm{~Hz} \\ \text { SW } & 8.763 \mathrm{ppm}\end{array}$

$\begin{array}{lr}\text { SW } & 8.763 \mathrm{ppm} \\ \text { FnMODE } & \text { QF }\end{array}$

F2 - Processing parameters
SI

$\begin{array}{ll}\text { SF } & 400.1300099 \\ \text { WDW } & \text { MHz }\end{array}$

SSB $\odot$

$\begin{array}{ll}\mathrm{LB} & \oplus \\ \mathrm{GB} & \odot \mathrm{Hz} \\ \mathrm{PC} & 0\end{array}$

PC $\quad 1.40$

F1 - Processing parameters

SI 2512

$\begin{array}{ll}\text { MC2 } & \text { QF } \\ \text { SF } & 400.1300099 \mathrm{MHz}\end{array}$

WDW $\quad \odot$

$\begin{array}{lll}\mathrm{LB} & 0 \\ \mathrm{~GB} & 0 & \mathrm{HZ} \\ \mathrm{GB} & 0\end{array}$ 
SSK-24-DR-GLU. BENZ-

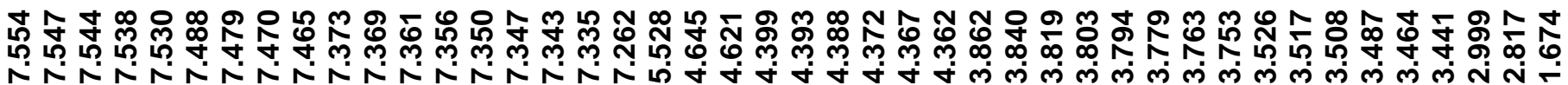

Current Data Parameters

NAME SSK-24-DR-GLU. BENZ-1H

EXPNO

PROCNO

F2 - Acquisition Parameters

Date_ 2019111

Time

14.18

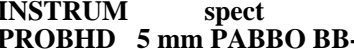

PULPROG zg30

TD 54274

NS 6

6 CDCl3

FIDRES $\quad 0.151522 \mathrm{H}$

AQ $\quad 3.2998593 \mathrm{sec}$

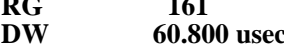

DE $\quad 6.50$ usec

TE $\quad 295.4 \mathrm{~K}$

D1 1.00000000

$========$ CHANNEL $\mathrm{f} 1 \mathrm{l}========$

NUC1 $1 \mathrm{H}$

$\begin{array}{ll}\text { P1 } & 14.75 \text { usec } \\ \text { PL1 } & -1.00 \mathrm{~dB}\end{array}$

PL1W 10.56200695 W

SFO1 $\quad 400.1324710 \mathrm{MHz}$

F2 - Processing parameters

$\begin{array}{ll}\text { SI } & 32768 \\ \text { SF } & 400.1300095 \\ \text { MHz }\end{array}$

WDW $\quad$ EM

SSB 0

$\begin{array}{lc}\text { LB } & 0.30 \mathrm{H} \\ \text { GB } & 0\end{array}$

PC $\quad 1.00$

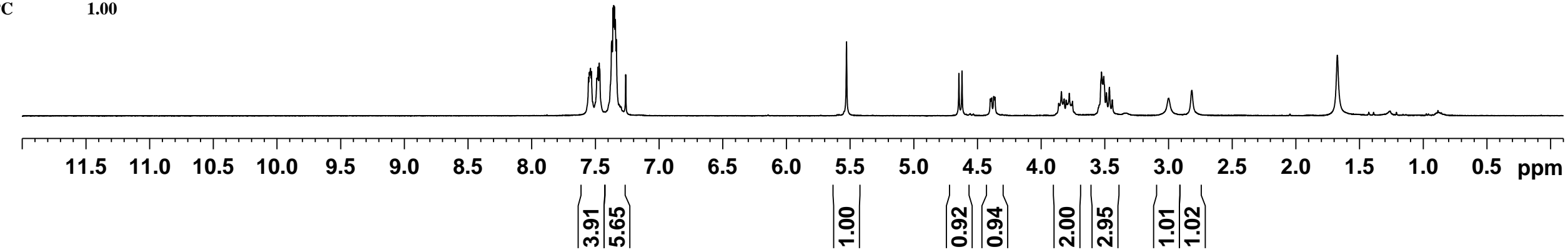




\section{SSK-24-DR-GLU. BENZ-13C}

Current Data Parameters NAME SSK-24-DR-GL

PROCNO

F2 - Acquisition Parameters

Date

INSTRUM 16.25

PROBHD $5 \mathrm{~mm}$ PABBO BB-

PULPROG 2 zgpg 30

SOLVENT ${ }_{\mathrm{CDCl}}$

$\begin{array}{lll}\text { NS } & 286 \\ \text { DS } & 0\end{array}$

$\begin{array}{lr}\text { SWH } & 26041.666 \mathrm{~Hz} \\ \text { FIDRES } & 0.397364 \mathrm{~Hz}\end{array}$

AQ $\quad 1.2582912 \mathrm{sec}$

$\begin{array}{ll}\text { RG } & 1030 \\ \text { DW } & 19.200 \text { usec }\end{array}$

$\begin{array}{ll}\text { DW } & 6.50 \text { usec } \\ \text { DE } & 296.1 \mathrm{~K}\end{array}$

D1 $\quad 1.00000000 \mathrm{sec}$

D11 $0.03000000 \mathrm{sec}$

$=======+$ CHANNEL $\mathrm{f} 1$ =======

$\begin{array}{ll}\text { NUC1 } & 13 \mathrm{C} \\ \text { P1 } & 8.50 \text { usec }\end{array}$

PL1 $\quad-2.00 \mathrm{~dB}$

$\begin{array}{ll}\text { PL1W } & 56.53121948 \mathrm{~W} \\ \text { SFO1 } & 100.6238364 \mathrm{MHz}\end{array}$

$=======$ CHANNEL $f 2=======$

${ }_{\text {NUC2 }}$ waltz16

PCPD2 80.00 usec

\begin{tabular}{ll} 
PL2 & $-1.00 \mathrm{~dB}$ \\
PL13 & $13.69 \mathrm{~dB}$ \\
\hline & $14.50 \mathrm{~dB}$
\end{tabular}

PL2W $\quad \begin{array}{ll}10.56200695 \mathrm{~W} \\ \text { PLWW }\end{array}$

PL13W $0.29767791 W$

SFO2 $\quad 400.1316005 \mathrm{MHz}$

F2 - Processing parameters

$\begin{array}{ll}\text { SI } & 32768 \\ \text { SF } & 100.6127690 \mathrm{MHz}\end{array}$

WDW $\quad 0$

$\begin{array}{ll}\text { LB } & 1.00 \mathrm{H} \\ \text { GB } & 0 \\ \text { PC } & 1.40\end{array}$

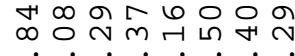

mim

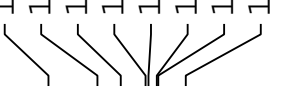

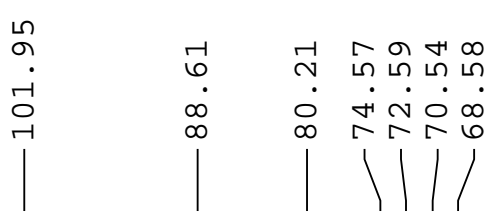

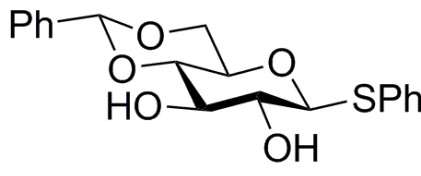

19

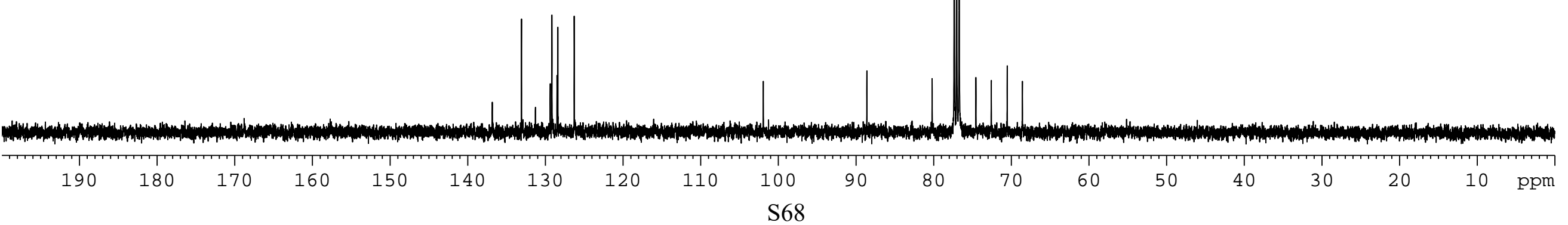




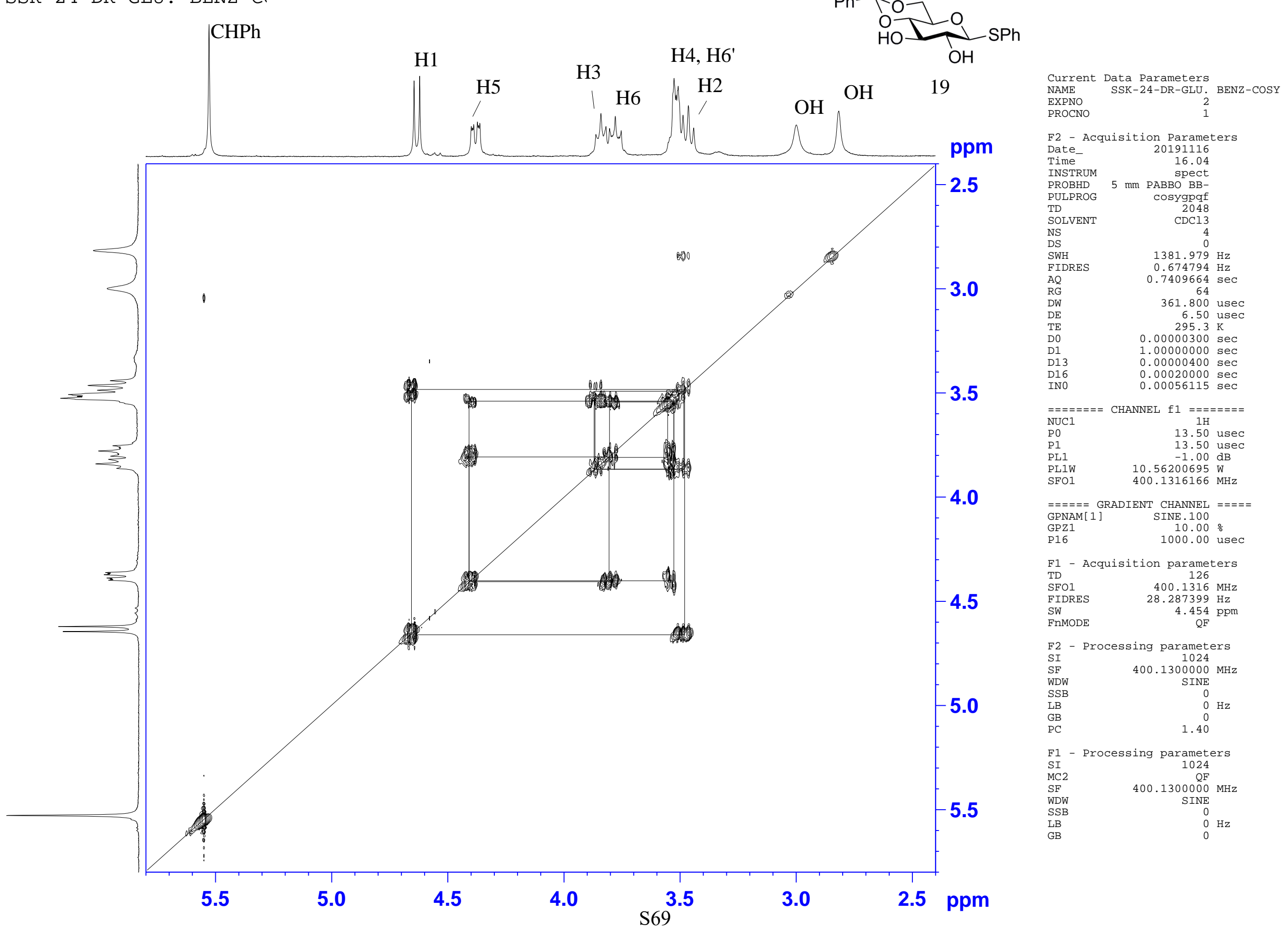


Current Data Parameters

Current Data Parameters
NAME

EXPNO

F2 - Acquisition Parameters

$\begin{array}{lr}\text { Date_- } & 20170711 \\ \text { Time } & 23.13\end{array}$

23.13
spect

PROBHD $5 \mathrm{~mm}$ PABBO BB

PULPROG

zg30
65536

TD

SOLVENT

NS

DS

16

$0.152588 \mathrm{~Hz}$
$\mathrm{H}$

AQ $\quad 3.2767999 \mathrm{sec}$

$\begin{array}{lr}\text { DW } & 50.00 \odot \text { usec } \\ \text { DE } & 6.50 \text { usec }\end{array}$

TE $296.9 \mathrm{~K}$

$\begin{array}{lr}\text { D1 } & 1.00000000 \mathrm{sec} \\ \text { TD } & 1\end{array}$

$========$ CHANNEL $\mathrm{f} 1$ ======== SF01 500.1330885 MHZ

NUC1 $1 \mathrm{H}$

$\begin{array}{lr}\text { P1 } & 13.50 \text { usec }\end{array}$

F2 - Processing parameters

SI - Processing parameters

SF $\quad 500.1300000 \mathrm{MHz}$

SSB
LB

$0.30 \mathrm{~Hz}$

PC $\quad 0 \quad 1.00$

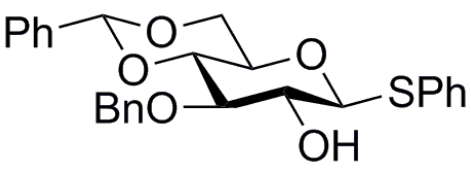

20

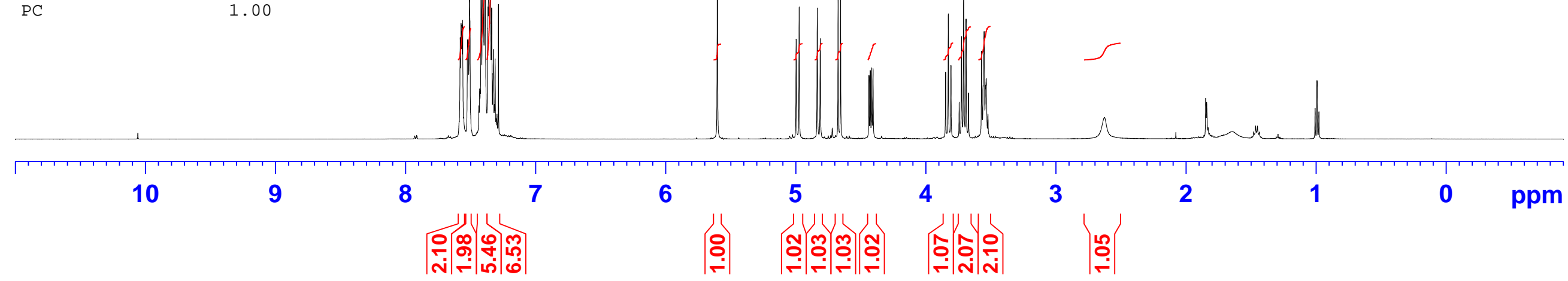


SSK - 11-AMB - 860-13C

Current Data Parameters

NAME SSK-11-AMB-860-13C

EXPNO

ROCNO 4
1

F2 - Acquisition Parameters

Date

Time

PROBHD

PULPROG

TD

SOLVENT

NS

DS

SWH

FIDRES

AQ
RG
DW

DE

TE

D11

TDO

======== CHANNEL f1 ======== SF01 125.7703637 MHz NUC1 $13 \mathrm{C}$

P1 8.90 usec

PLW1 $103.00000000 \mathrm{~W}$

$=======$ CHANNEL f2 ========

SF02 500.1320005 MHz

NUC2

CPDPRG [2

PCPD2

PLW12

waltz16

80 usec

13. 00000000

$0.37020001 \mathrm{~W}$

F2 - Processing parameters

$\begin{array}{lr}\text { SI } & 32768 \\ \text { SF } & 125.7577640 \mathrm{MHz}\end{array}$

WDW

SSB

GB

$\mathrm{PC}$

$1.00 \mathrm{~Hz}$

1.40

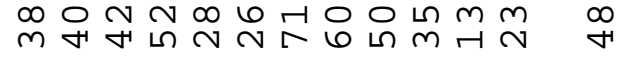

○ิ

苞
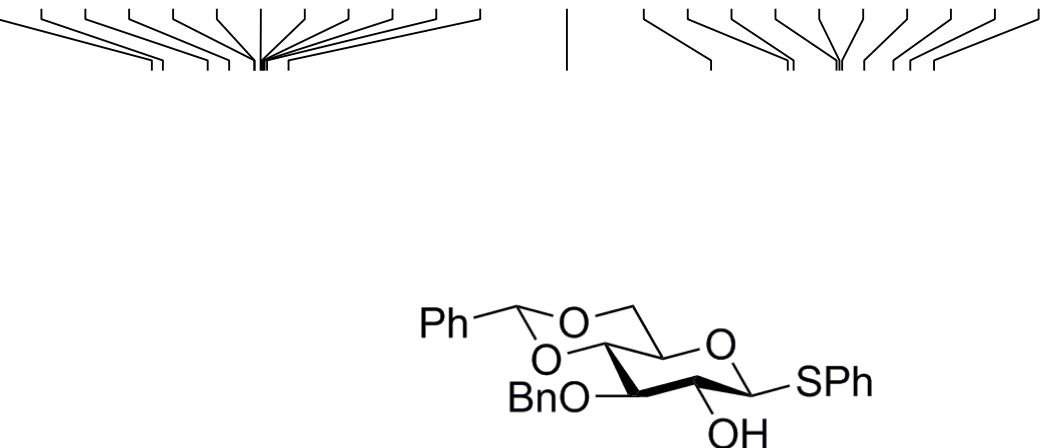


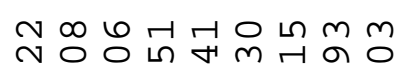

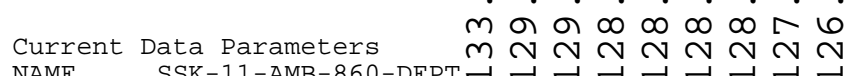
NAME SSK-11-AMB-860-DEPT

EXPNO

F2 - Acquisition Parameters

Time-

TNSTRUM

PROBHD

PULPROG

SOLVENT

NS

SWH

IDRES

$\begin{array}{lr}\text { FIDRES } & 0.307637 \mathrm{~Hz} \\ \text { AQ } & 1.6252928 \mathrm{sec} \\ \text { RG } & 197.27\end{array}$

24.800 usec

6.50 usec

145.0000000

145.0000000

$\begin{array}{ll}\text { D1 } & 1.000000000 \mathrm{sec} \\ \text { D2 } & 0.00344828 \mathrm{sec} \\ \text { D12 } & 0.00002000 \mathrm{sec}\end{array}$

$\begin{array}{ll}\text { D1 } & 1.000000000 \mathrm{sec} \\ \text { D2 } & 0.00344828 \mathrm{sec} \\ \text { D12 } & 0.00002000 \mathrm{sec}\end{array}$

$=======$ CHANNEL $\mathrm{f} 1$ ========

SF01 $125.7678486 \mathrm{MHz}$

NUC1

P1

P13

PLWO

PLW1

SPOAL5

SPOFFS

SPW5

$====2=\mathrm{CHAN} E \mathrm{~L}$

$\begin{array}{cc}\text { SF02 } & 500.1315995 \mathrm{MHz}\end{array}$

NUC2 $1 \mathrm{H}$

$\begin{array}{lr}\text { CPDPRG } 2 & \text { waltz16 } \\ \text { P3 } & 13.50 \text { usec }\end{array}$

PCPD2 20.00 usec

$\begin{array}{lr}\text { PLW2 } & 13.00000000 \mathrm{~W} \\ \text { PLW12 } & 0.37020001 \mathrm{~W}\end{array}$

F2 - Processing parameters

SI $\quad 32768$

SF $\quad 125.7577890 \mathrm{MHz}$

$\begin{array}{lll}\mathrm{SSB} & 0 & 1.00 \mathrm{~Hz}\end{array}$

GB 0

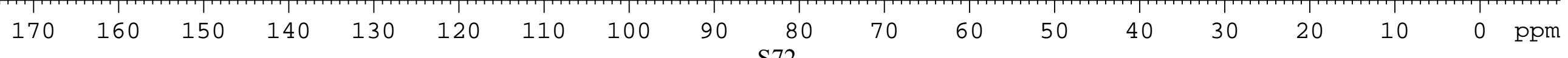

○े

$\infty \infty \infty \pi+\frac{N}{2}$

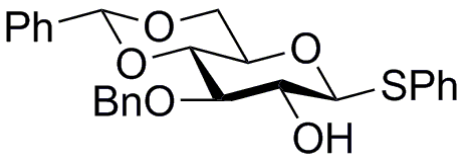

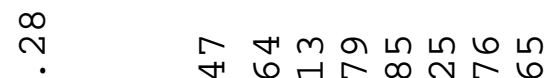

$1 \backslash 111$

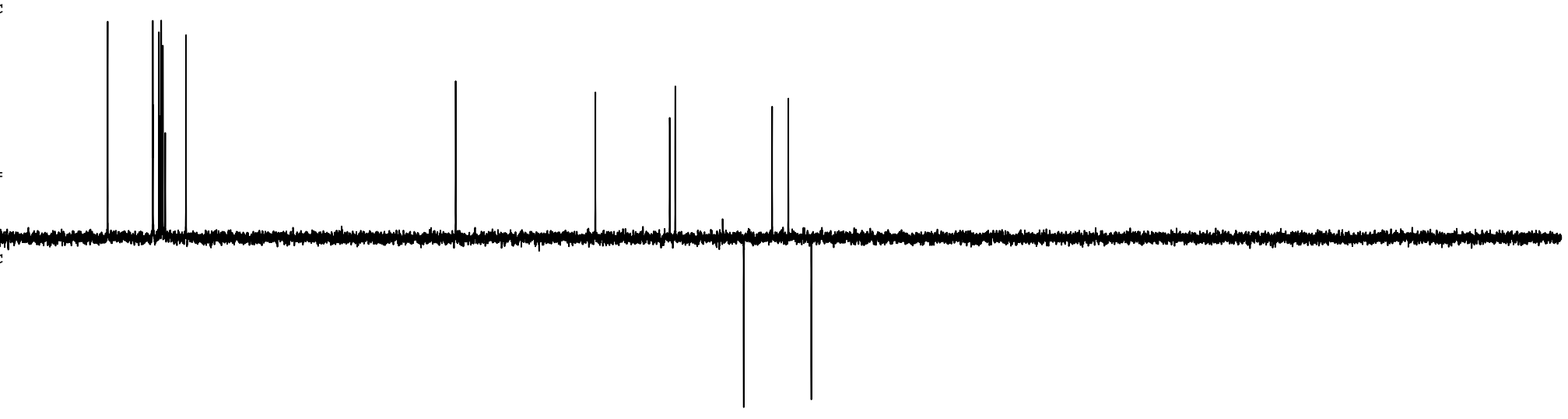




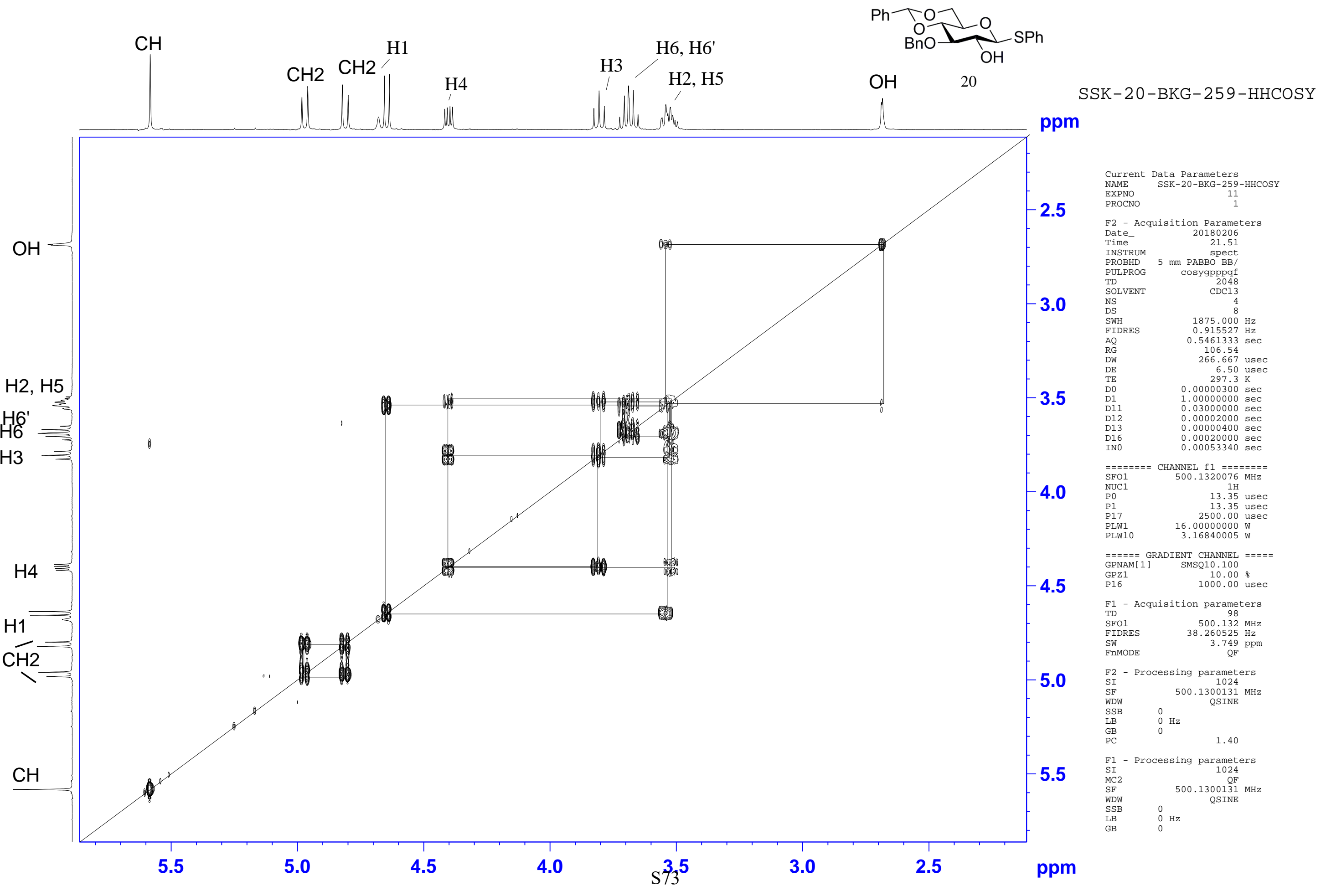


Current Data Parameters

SSK-11-AMB-BENZ-DONOR-1H

EXPNO 3
1

F2 - Acquisition Parameters

Time

INSTRUM

PULPROG

20181113

SOLVENT

65536

NS

$\begin{array}{lr} & 10000.000 \mathrm{~Hz} \\ \text { FIDRES } & 0.152588 \mathrm{~Hz}\end{array}$

AQ $\quad 3.2767999 \mathrm{sec}$

DW $\quad 50.000$ usec

DE $\quad 6.50$ usec

D1 $1.00000000 \mathrm{sec}$

TDQ

$=======$ CHANNEL $\mathrm{f} 1$ ========

SF01 $500.1330885 \mathrm{MHz}$

NUC1 $13.1 \mathrm{H}$ usec

13.35 use
PLW1 $16.00000000 \mathrm{~W}$

F2 - Processing parameters

$\begin{array}{lr}\text { SI } & 65536 \\ \text { SF } & 500.1300134 \mathrm{MHz}\end{array}$

WDW

LB $\quad 0 \quad 0.30 \mathrm{~Hz}$

$\begin{array}{lll}\text { GB } & \odot & 1.00\end{array}$

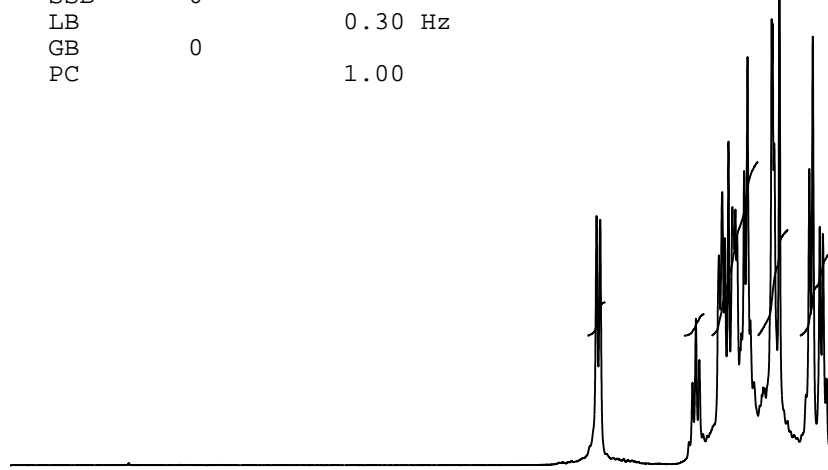

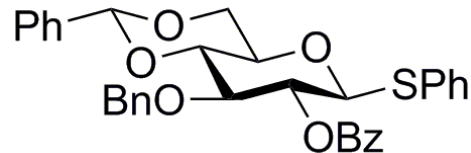

6

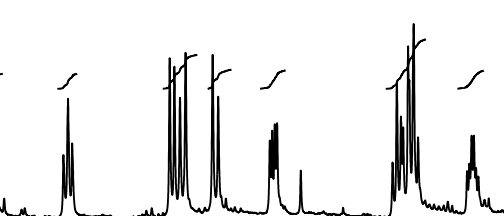

$\begin{array}{llll}10.0 & 9.5 & 9.0 & 8.5\end{array}$

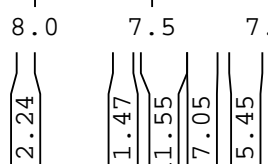

6.5

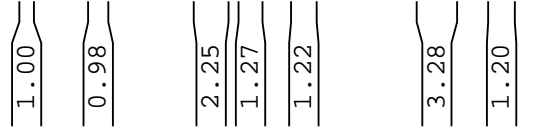




\begin{tabular}{lc|} 
& \\
Current & Data Parameters \\
NAME & SSK-11-AMB-866-13C \\
EXPNO & 9 \\
PROCNO & 1
\end{tabular}
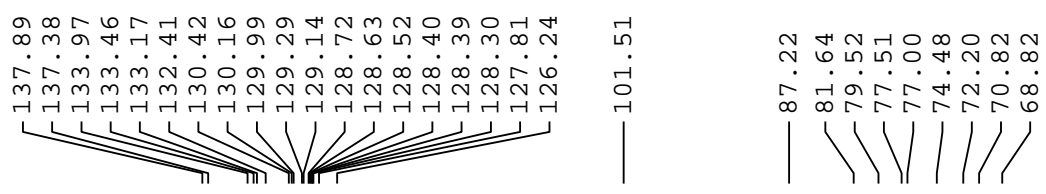

F2 - Acquisition Parameters

Date_ 20170711

$\begin{array}{lr}\text { Time } & 23.22 \\ \text { INSTRUM } & \text { spect }\end{array}$

PROBHD $5 \mathrm{~mm}$ PABBO BB/

$\begin{array}{lr}\text { PULPROG } & \text { Zgpg30 } \\ \text { TD } & 65536\end{array}$

TD

SOLVENT

NS

$\mathrm{CDCl}$
61

DS

SWH 29761.904 Hz

FIDRES $\quad 0.454131 \mathrm{~Hz}$

AQ $\quad 1.1010048 \mathrm{sec}$

RG

DW

$\mathrm{DE}$

TE

D1

197.27

16.800 usec

6.50 usec

$297.4 \mathrm{~K}$

$1.000 \odot \odot \odot \odot \odot \mathrm{sec}$

$\odot .03000 \odot \odot \odot \mathrm{sec}$

TD९ 1

======== CHANNEL f1 ======== $\begin{array}{ll}\text { SF01 } & 125.7703637 \mathrm{MHZ}\end{array}$

NUC1 $13 \mathrm{C}$

P1 8.90 usec

PLW1 $103.00000000 \mathrm{~W}$

=====ニ== CHANNEL $\mathrm{f} 2$ =====ニ== SF02 500.1320005 MHZ NUC2 $\quad 1 \mathrm{H}$

PCPD2 $\quad 80.00$ usec

PLW2 $13.00000000 \mathrm{~W}$

PLW12 $\quad 0.37020001 \mathrm{~W}$

F2 - Processing parameters

SI $\quad 32768$

SF $\quad 125.7577648 \mathrm{MHz}$

$\begin{array}{lll}\text { SSB } & 0 & 1.00 \mathrm{~Hz} \\ \text { LB } & & \end{array}$

$\begin{array}{lll}\mathrm{GB} & 0 & 1.40\end{array}$

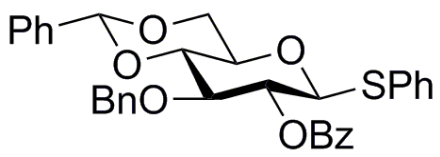

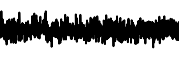


SSK - 11 - AMB - 866 - DEPT

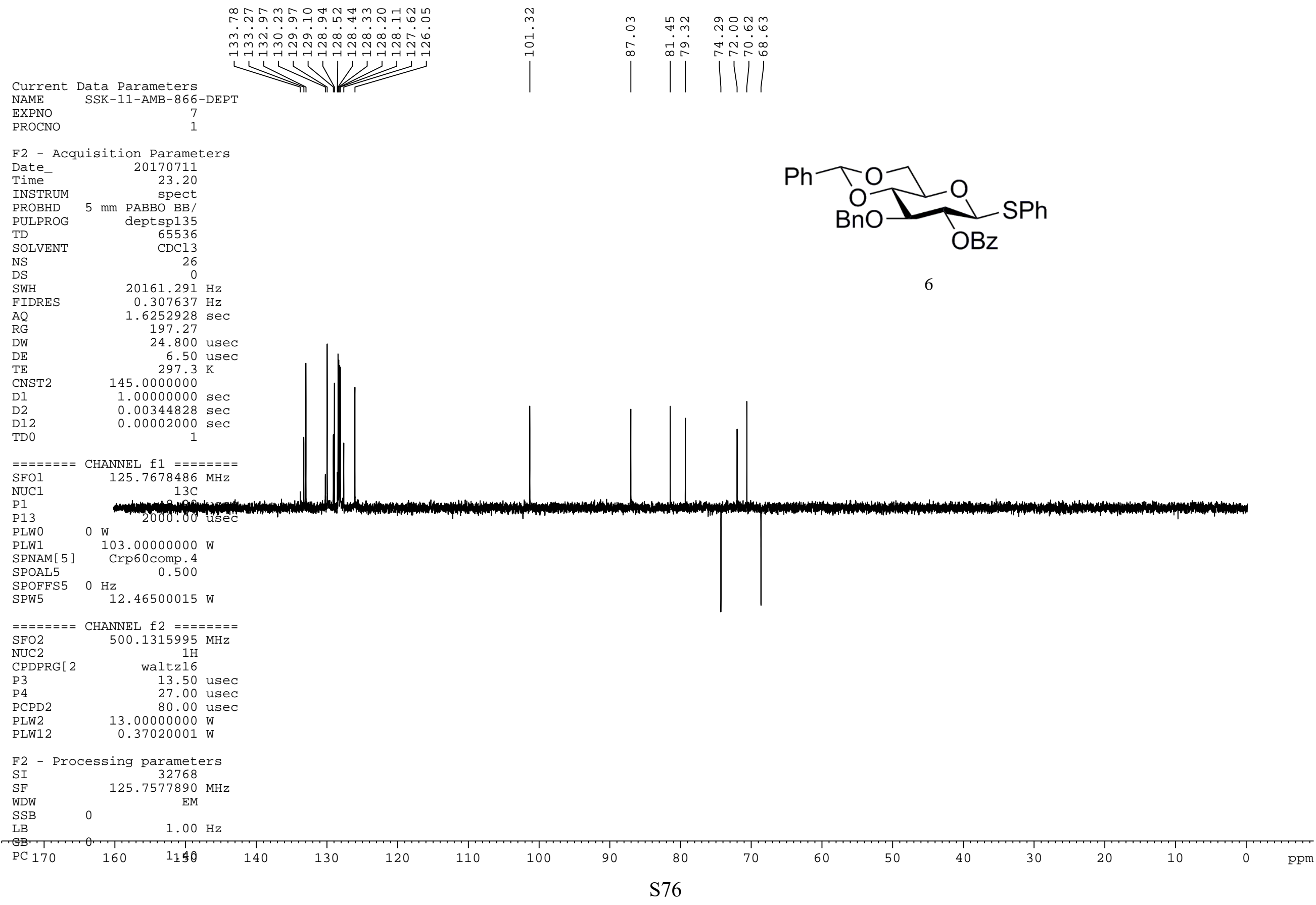


SSK - 11 - AMB - 866-COSY

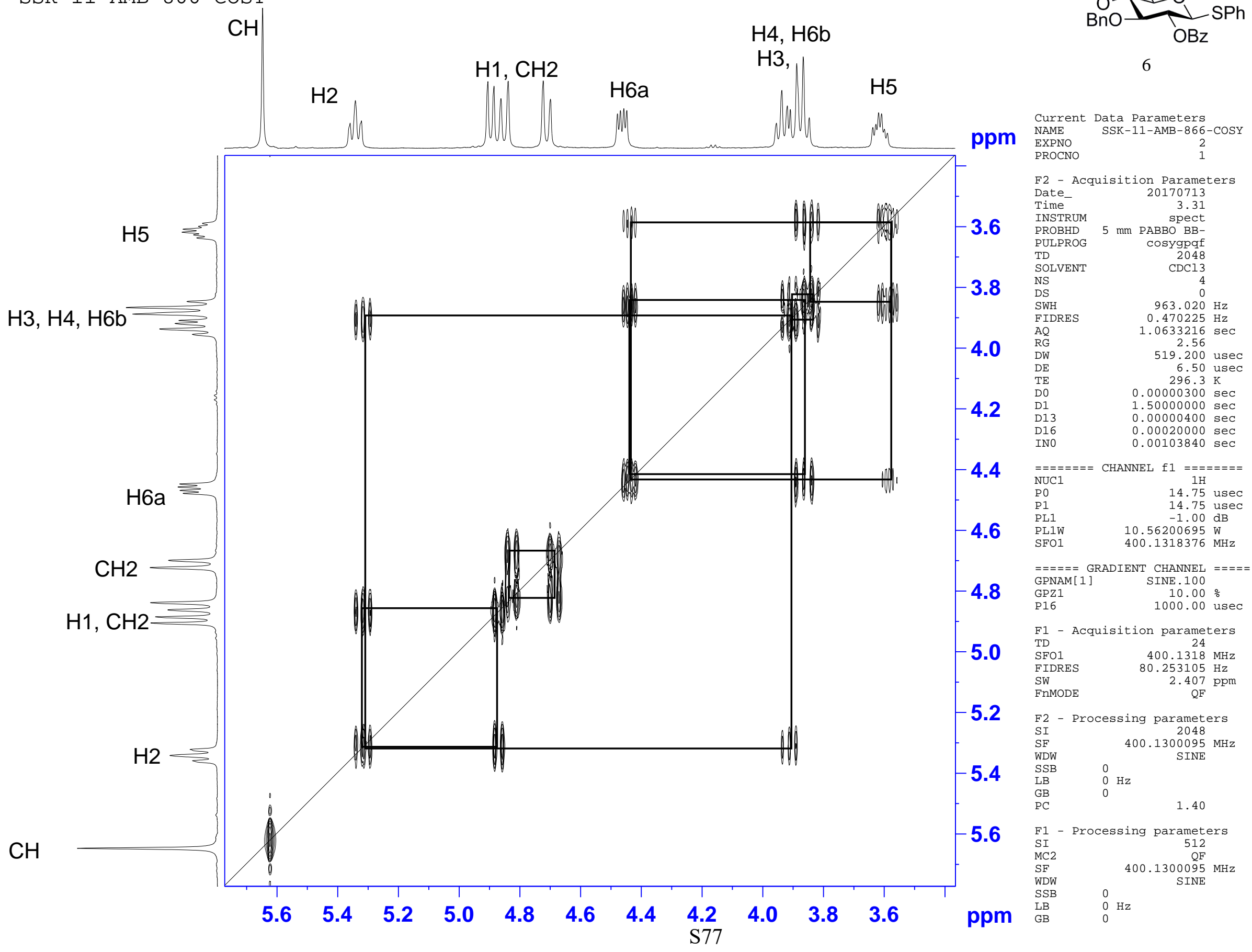


Current Data Parameters

SSK-11-AMB-GLU-4-OH-1H

EXPNO

1
1

F2 - Acquisition Parameters

Date_ 20181108

$\begin{array}{ll}\text { Time } & 23.00 \\ \text { INSTRUM } & \text { spect }\end{array}$

PROBHD $5 \mathrm{~mm}$ PABBO BB-

$\begin{array}{lr}\text { PULPROG } & \text { Zg30 } \\ \text { TD } & 54274\end{array}$

$\begin{array}{ll}\text { TD } & 54274 \\ \text { SOLVENT } & \text { CDCl3 }\end{array}$

NS

20
0

DS

$\begin{array}{lr}\text { SWH } & 8223.685 \mathrm{~Hz} \\ \text { FIDRES } & 0.151522 \mathrm{~Hz} \\ \text { AQ } & 3.2998593 \mathrm{sec}\end{array}$

$\begin{array}{lr}\text { SWH } & 8223.685 \mathrm{~Hz} \\ \text { FIDRES } & 0.151522 \mathrm{~Hz} \\ \text { AQ } & 3.2998593 \mathrm{sec}\end{array}$

RG $\quad 161$

60.800
DE

TE $297.6 \mathrm{~K}$

$\begin{array}{lr}\text { D1 } & 1.0000000 \odot \mathrm{sec} \\ \text { TD० } & 1\end{array}$

$=======$ CHANNEL f1 ========

$\begin{array}{ll}\mathrm{NUC1} 1 & 1 \mathrm{H} \\ \mathrm{P} 1 & 14.75 \mathrm{usec}\end{array}$

$\quad 10.56200695 \mathrm{~W}$

$\begin{array}{ll}\text { PL1W } & 10.56200695 \mathrm{~W} \\ \text { SF01 } & 400.1324710 \mathrm{MHz}\end{array}$

F2 - Processing parameters

$\begin{array}{lr}\text { SI } & 32768 \\ \text { SF } & 400.1299786 \\ \text { WHZ }\end{array}$

WDW $\quad 400.1299786 \mathrm{MHz}$

$\begin{array}{lll}\text { SSB } & 0 & 0.30 \mathrm{~Hz}\end{array}$

$\begin{array}{lll}\mathrm{GB} & \odot & 1.00\end{array}$

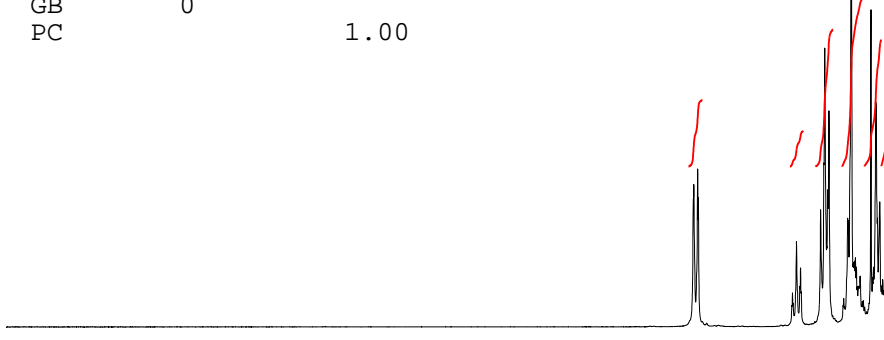

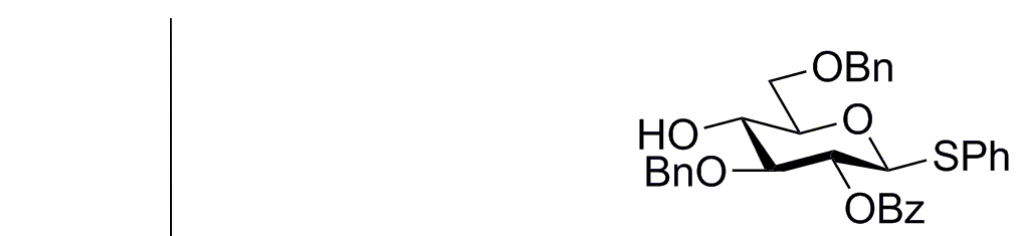

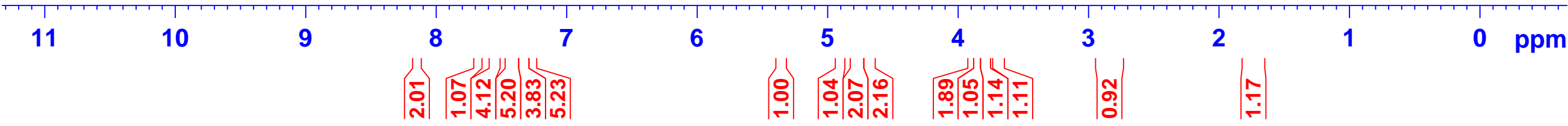




$\begin{array}{lc} & \\ \text { Current } & \text { Data Parameters } \\ \text { NAME } & \text { SSK-11-AMB-GLU-4-OH-13C } \\ \text { EXPNO } & 2 \\ \text { PROCNO } & 1\end{array}$

$$
\text { EXPNO }
$$

F2 - Acquisition Parameters

Date -

20181108
23.01

INSTRUM

$5 \mathrm{~mm}$ PABBO BB-

PULPROG 2 Zgpg30

TD

zgpg30
65536

SOLVENT

NS

DS

FIDRES

AQ

$\mathrm{DW}$

DE

TE

D1
D11
TD0 NUC1 $13 \mathrm{C}$

PL1 18.50 usec PL1W $56.53121948 \mathrm{~W}$ SF01 $100.6238364 \mathrm{MHz}$

$========$ CHANNEL $\mathrm{f} 2$ ======== CPDPRG [2 waltz16

PCPD2 80.00 usec

PL2 -1.00 dB

$\begin{array}{ll}\text { PL12 } & 13.69 \mathrm{~dB} \\ \text { PL13 } & 14.50 \mathrm{~dB}\end{array}$

PL2W $\quad 10.56200695 \mathrm{~W}$

PL12W $\quad 0.35871249 \mathrm{~W}$

SF02 $\quad 0.29767781 \mathrm{~W}$

F2 - Processing parameters

$\begin{array}{lr}\text { SI } & 32768 \\ \text { SF } & 100.6127477 \mathrm{MHz}\end{array}$

WDW $\quad 100.6127477$ MHz

$\begin{array}{lll}\text { SSB } & \odot & 1.00 \mathrm{~Hz} \\ \text { LB } & 0 & \end{array}$

$\begin{array}{lll}\mathrm{GB} & 0 & 1.40\end{array}$

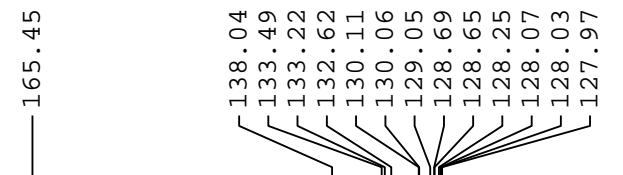

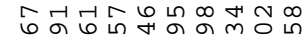

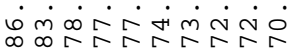

11111

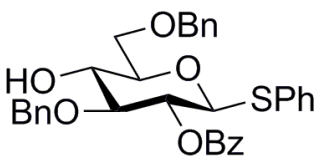

21 


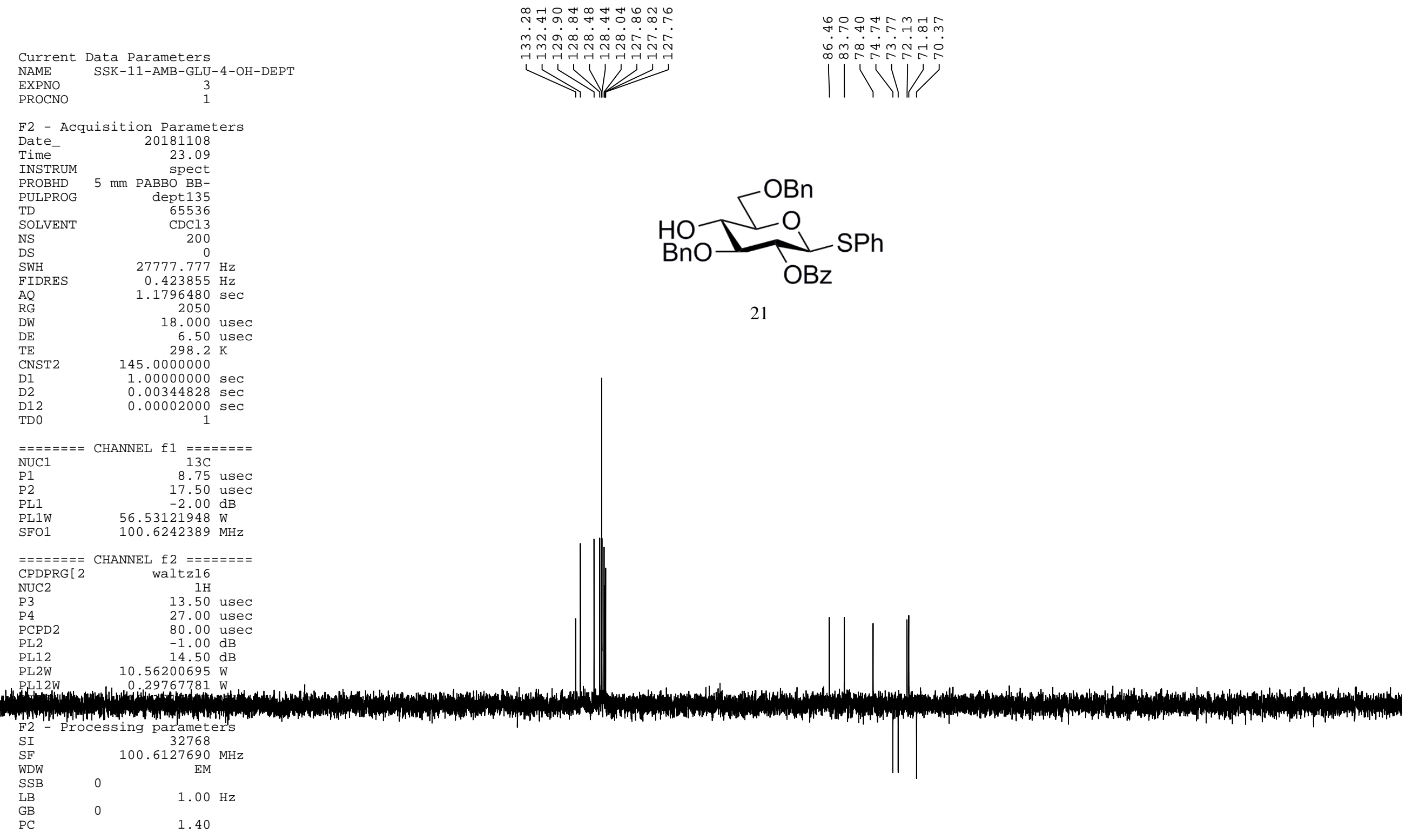


SSK - 11 - AMB - GLU - 4-OH - COSY

H6b, $\mathrm{H} 4$

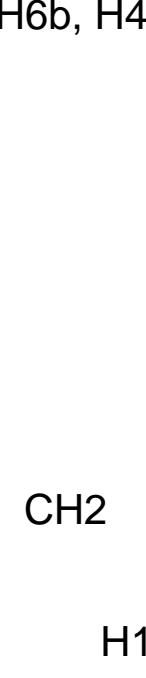

$\mathrm{H} 1$

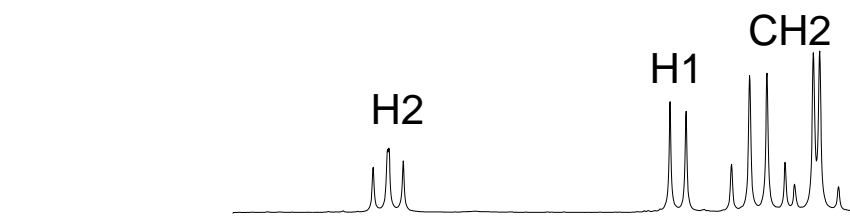

H6a, H6b, H4

Net $\mathrm{H}^{\mathrm{H}}$

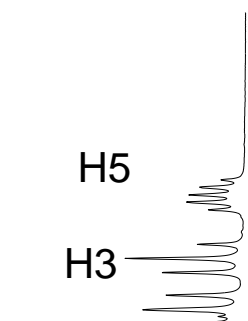

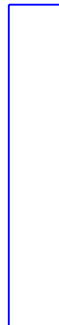
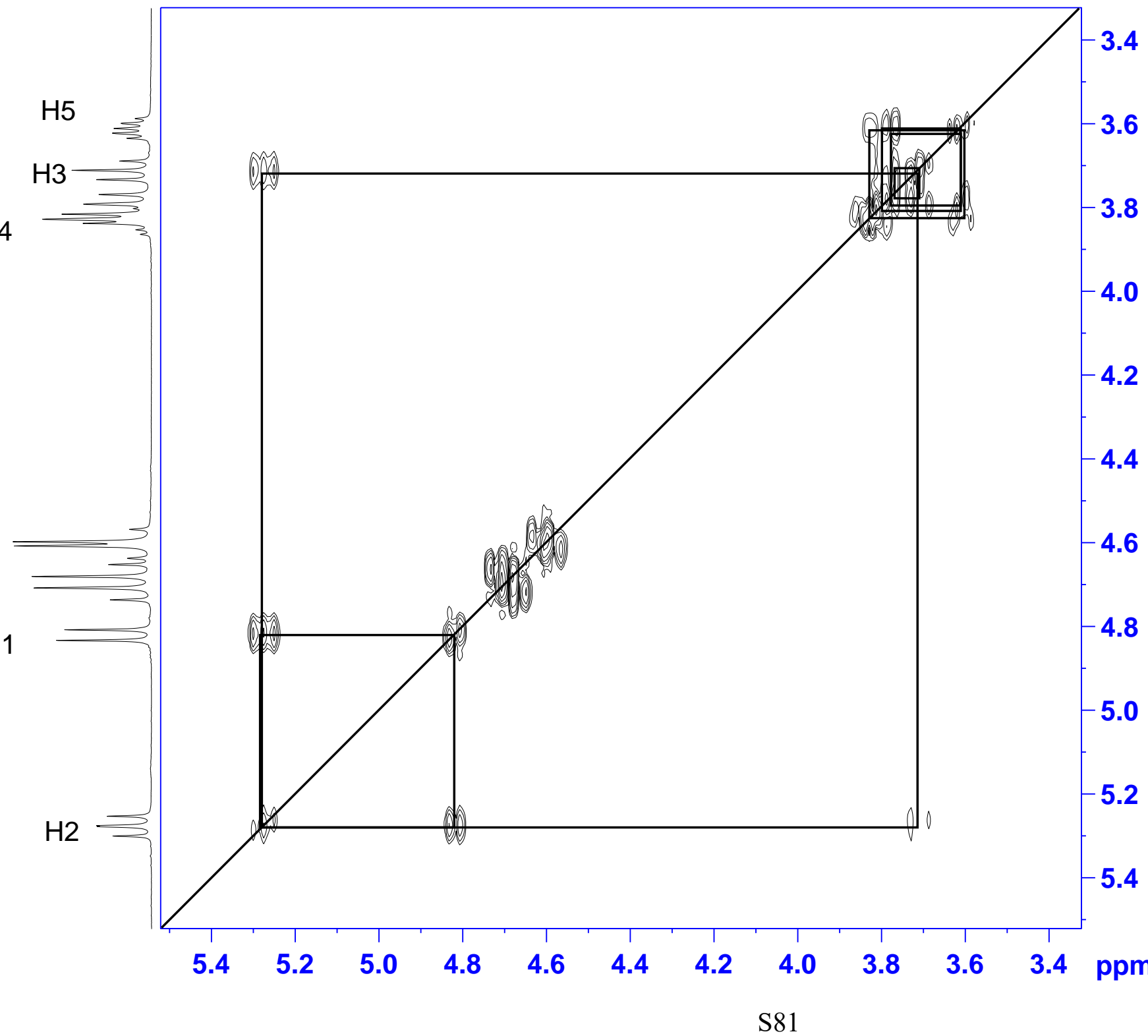

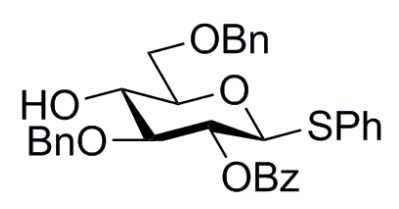

21

ppm

$-3.4$

3.6

3.8

$-4.0$

4.2

4.4

$-4.6$

4.8

5.0

5.2

5.4

pm
Current Data Parameters
NAME SSK-11-AMB-GLU-4-OH-COSY EXPNO

F2 - Acquisition Parameters

Date-

Time

$5 \mathrm{~mm}$ PABBO $\mathrm{SB}-$

PULPROG

cosygpqf

SOLVENT

NS

SWH

$\mathrm{AQ}$
$\mathrm{RG}$
$\mathrm{DW}$
$\mathrm{DE}$

$\mathrm{DW}$
$\mathrm{DE}$
$\mathrm{TE}$

D0
D1
D13

INO

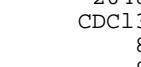

$3424.657 \mathrm{~Hz}$ $1.672196 \mathrm{~Hz}$ $0.2990080 \mathrm{sec}$ 146.000 usec
6.50 usec $297.0 \mathrm{~K}$ $0.00000300 \mathrm{sec}$ $1.00000000 \mathrm{sec}$
$0.00000400 \mathrm{sec}$ $0.00020000 \mathrm{sec}$ $0.00029200 \mathrm{sec}$

$=======$ CHANNEL $\mathrm{f} 1========$
NUC1 $1 \mathrm{H}$ $\begin{array}{lr}\text { NUC1 } & 1 \mathrm{H} \\ \text { P0 } & 13.50 \text { usec } \\ \text { P1 } & 13.50 \text { usec } \\ \text { PL1 } & -1.00 \mathrm{~dB} \\ \text { PL1W } & 10.56200695 \mathrm{~W} \\ \text { SF01 } & 400.1316202 \mathrm{MHz}\end{array}$

$=====$ GRADIENT CHANNEL $==$ GPNAM [1] SINE.100 P16 $1000.00 \%$

P16 - Acquisition paraneters

F1 - Acquisition parameters

TD01 $\quad 400.1316 \mathrm{MHz}$

FIDRES $\quad 53.510101 \mathrm{~Hz}$

$\begin{array}{lr}\text { SW } & 8.559 \mathrm{ppm} \\ \text { FnMODE } & \text { QF }\end{array}$

F2 - Processing parameters

$\begin{array}{lr}\text { SI } & 1024 \\ \text { SF } & 400.1300095 \mathrm{MHz}\end{array}$

WDW

$\begin{array}{ll}\text { SSB } & \odot \\ \text { LB } & \odot \\ \text { GB } & \odot\end{array}$

$\begin{array}{lll}\mathrm{GB} & \mathrm{P} & 1.40\end{array}$

F1 - Processing parameters

$\begin{array}{lc}\text { MC2 } & \text { QF } \\ \text { SF } & 400.1300095 \mathrm{MHz} \\ \text { WDW } & \text { SINE }\end{array}$

$\begin{array}{lll}\text { WDW } & & \\ \text { SSB } & \ominus \\ \text { LB } & 0 \\ \text { GB } & 0\end{array}$ 
Current Data Parameters

NAME SSK-11-AMB-1040-1H

EXPNO 1

PROCNO

F2 - Acquisition Parameters

Date_ 20180227

$\begin{array}{lr}\text { Time } & 11.34\end{array}$

INSTR $5 \mathrm{~mm}$ spect

PULPROG 5 TI PABBO BB-

$\begin{array}{lr}\text { PULPROG } & \text { Zg30 } \\ \text { TD } & 54274\end{array}$

SOLVENT CDCl3

$\begin{array}{lr}\text { NS } & \text { CDC13 } \\ \text { DS } & 6\end{array}$

$8223.685 \mathrm{~Hz}$

$\begin{array}{lr}\text { SWH } & 8223.685 \mathrm{~Hz} \\ \text { FIDRES } & 0.151522 \mathrm{~Hz} \\ \text { AQ } & 3.2998593 \mathrm{sec}\end{array}$

$\begin{array}{lr}\text { AQ } & 3.2998593 \\ \text { RG } & 90.5\end{array}$

$\begin{array}{lr}\text { RG } & 90.5 \\ \text { DW } & 60.800 \text { usec }\end{array}$

DE $\quad 6.50$ use

D1 $1.00000000 \mathrm{sec}$

TD० 1

$=======$ CHANNEL $\mathrm{f} 1 \mathrm{l}=======$
NUC1
$1 \mathrm{H}$

P1 14.75 use

PL1 $\quad-1.00 \mathrm{~dB}$

$\begin{array}{ll}\text { PL1W } & 10.56200695 \mathrm{~W} \\ \text { SF01 } & 400.1324710 \mathrm{MHz}\end{array}$

F2 - Processing parameters

$\begin{array}{lr}\text { SI } & 32768 \\ \text { SF } & 400.1300190 \mathrm{MHz}\end{array}$

$\begin{array}{lr}\text { SF } & 400.1300190 \mathrm{MHz} \\ \text { WDW } & \text { EM }\end{array}$

SSB $\quad 0$

LB $\quad 0.30 \mathrm{~Hz}$

$\begin{array}{lll}\mathrm{GB} & \odot & 1.00\end{array}$

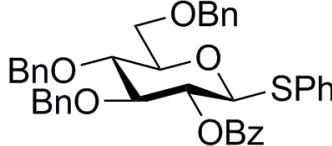

22

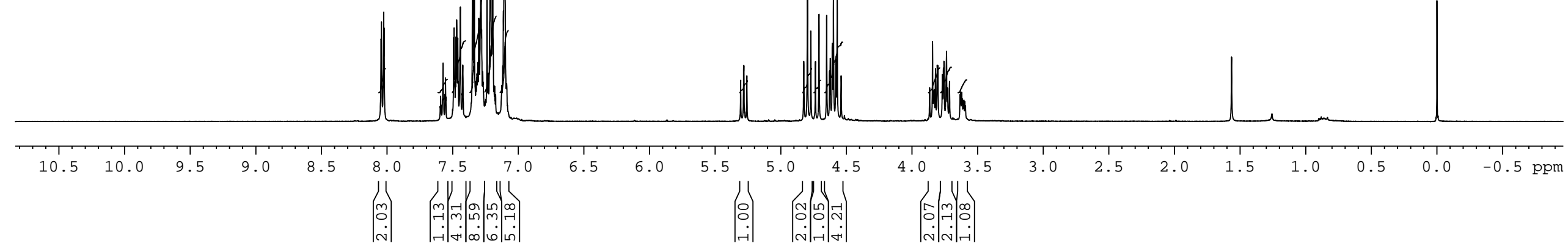


Current Data Parameters

$B-1040-13 C$

EXPNO

3
1

F2 - Acquisition Parameters

Date__ 20180227

Time $\quad 11.35$

spect
INSTRUM $5 \mathrm{~mm}$ PABBO

PULPROG $\quad$ zgpg30

\begin{tabular}{lr} 
Tgpg3 \\
TD & 65536 \\
\hline
\end{tabular}

SOLVENT

NS

DS

FIDRES

$\mathrm{AQ}$
$\mathrm{RG}$

RG

DW

TE

D1

D11

=ニニ=ニ= NUC1

$\mathrm{P} 1$

PL1

PL1W

SF01

$\mathrm{CDCl} 3$

$26041.666 \mathrm{~Hz}$

$0.397364 \mathrm{~Hz}$

$1.2582912 \mathrm{sec}$

2050

19.200 usec 6.50 usec

$1.00000000 \mathrm{sec}$

$0.03000000 \mathrm{sec}$

CHANNEL f1 ======== $13 \mathrm{C}$

8.50 usec
$2.00 \mathrm{~dB}$

$56.53121948 \mathrm{~W}$

$100.6238364 \mathrm{MHz}$

$========$
CPDPRG $[2$ wanNEL f $2========$
walt $z 16$

NUC2 $1 \mathrm{H}$

PCPD2 80.00 usec

PL13

PL2W

PL12W

PL13W

$13.69 \mathrm{~dB}$

$10.56200695 \mathrm{~W}$

$10.56200695 \mathrm{~W}$

$0.35871249 \mathrm{~W}$

$400.1316005 \mathrm{MHz}$

F2 - Processing parameters

$\begin{array}{lcc}\text { F2 } & \text { - Processing parameters } \\ \text { SI } & 32768 \\ \text { SF } & & 100.6127482 \\ \text { WDW } & & \text { EM } \\ \text { SSB } & \odot & \end{array}$

LB
GB

0

$1.00 \mathrm{~Hz}$

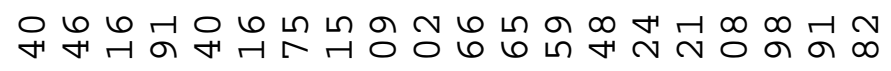

.

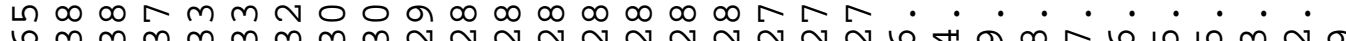

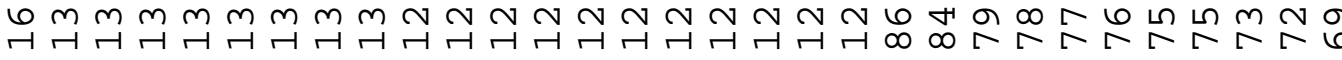
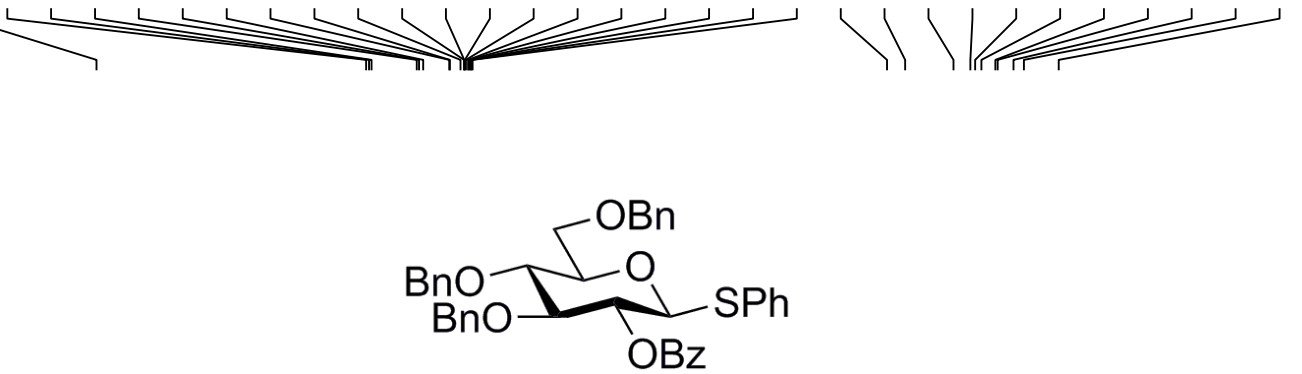

22

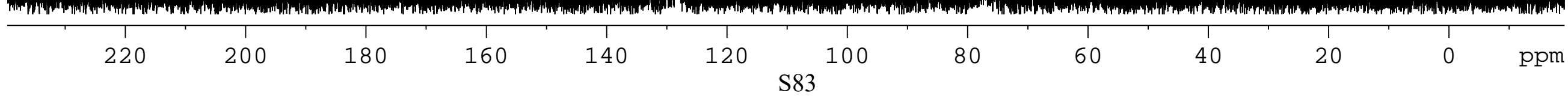


SSK - 11 - AMB - 1040 - DEPT

$\begin{array}{lc}\text { Current } & \text { Data Parameters } \\ \text { NAME } & \text { SSK-11-AMB-1040-DEPT } \\ \text { EXPNO } & 2 \\ \text { PROCNO } & 1\end{array}$

F2 - Acquisition Parameters

Date-

INSTRUM

PROBHD

PULPROG

11.38
spect
$5 \mathrm{~mm}$ РАВB BB-

TD SOLVENT

NS

DS

SWH
FIDRES

AQ

$\mathrm{RG}$

DW

DE

CNST2

D1

D2
D12

TD0

$========$ CHANNEL

NUC1

$\mathrm{P} 1$
$\mathrm{P} 2$

PL1

PL1W CPDPRG [2 2 waltz16

NUC2 2

$\begin{array}{ll}\mathrm{P} 3 & 1 \mathrm{H} \\ \mathrm{P} 4 & 14.75 \mathrm{usec}\end{array}$

P4 29.50 usec

PCPD2 80.00 usec

PL2

PL12

PL2W
PL12W

$-1.00 \mathrm{~dB}$

$10.56200695 W$
$0.35871249 W$

$0.35871249 \mathrm{~W}$
$400.1316005 \mathrm{MHz}$

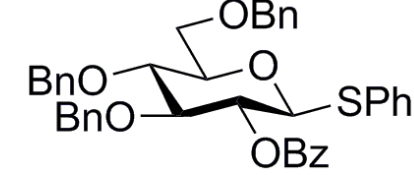

22

F2 - Processing parameters

$\begin{array}{ll}\text { SI } & 32768 \\ \text { SF } & 100.6127690 \mathrm{MHz}\end{array}$

$\begin{array}{lll}\text { SSB } & \odot & 3.00 \mathrm{~Hz}\end{array}$

$\begin{array}{lll}\mathrm{GB} & 0 & 1.40 \\ \mathrm{PC} & \end{array}$

$210 \quad 200 \quad 190 \quad 180$

$170 \quad 160$

150

140

120

110 
SSK-11-AMB-1040-HHCOSY

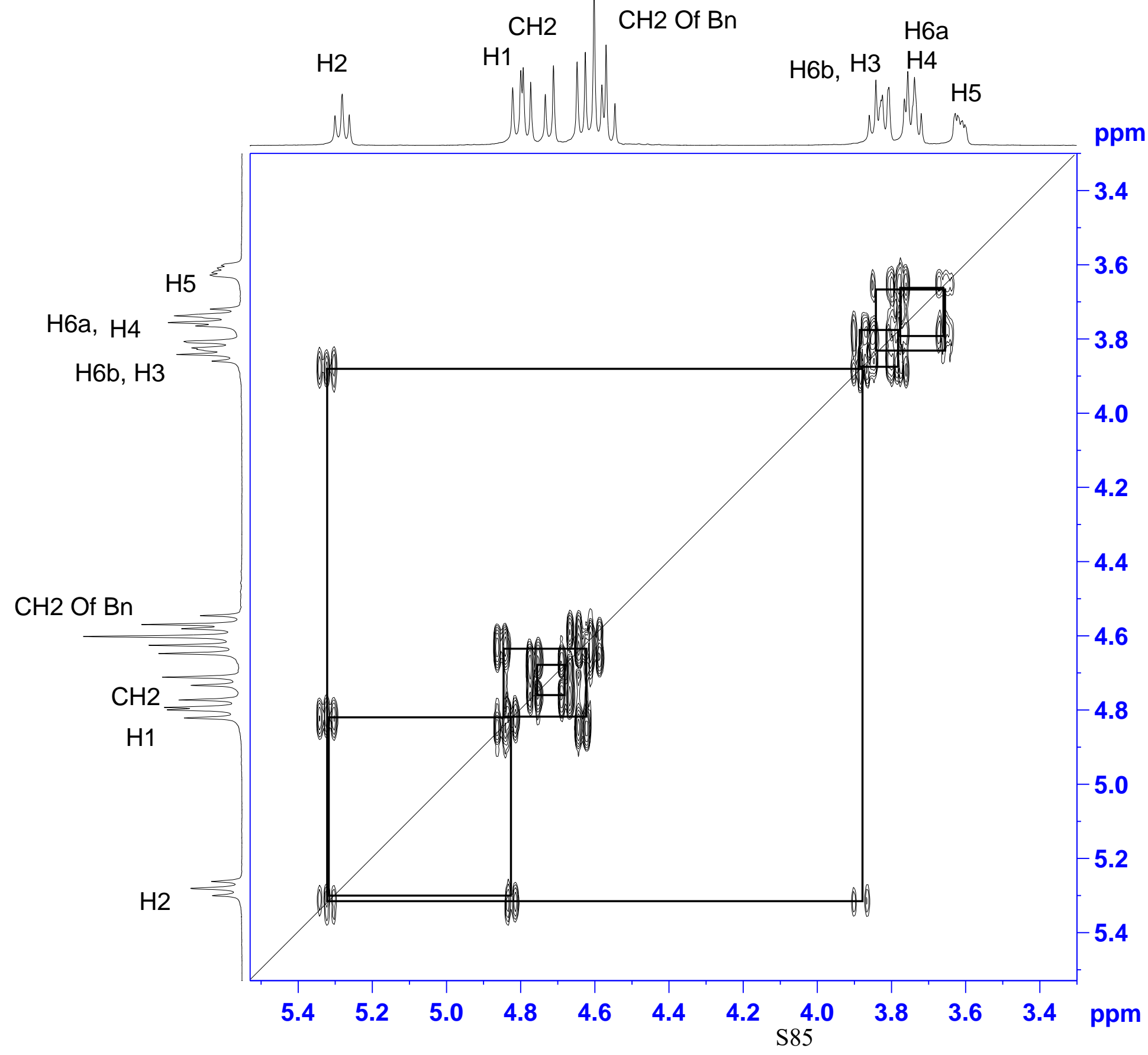

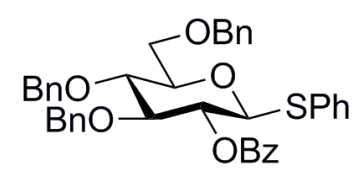

22

Current Data Parameter

-1055-HHCOSY EXPNO
PROCNO

F2 - Acquisition Parameters

Date_-

21.12
Time

spect

PULPROG COSygpppq

SOLVENT

NS

$\begin{array}{lr}\text { DS } & \stackrel{\ominus}{ } \\ \text { SWH } & 2020.474 \mathrm{~Hz} \\ \text { FIDRES } & 0.986560 \mathrm{~Hz}\end{array}$

$\begin{array}{lr}\text { AQ } & 0.986560 \mathrm{~Hz} \\ \text { RQ } & 0.5068117 \mathrm{sec}\end{array}$

$\begin{array}{lr}\text { RG } & 61.42 \\ \text { DW } & 247.467 \text { usec } \\ \text { DE } & 6.50 \text { usec }\end{array}$

DE

D 0
D1
D11

$\begin{array}{ll}\text { D11 } & 1.000000000 \mathrm{sec} \\ \mathrm{D} & 0.03000000 \mathrm{sec}\end{array}$

$\begin{array}{ll} & 0.03000000 \mathrm{sec} \\ \mathrm{D} 12 & 0.00002000 \mathrm{sec}\end{array}$

$\begin{array}{ll}\text { D16 } & 0.00020000 \mathrm{sec} \\ \text { INO } & 0.00049500 \mathrm{sec}\end{array}$

$========$ CHANNEL $\mathrm{f} 1 \mathrm{1}=======$

SF01 $500.1317553 \mathrm{MHZ}$

$\begin{array}{lr}\text { NUC1 } & 1 \mathrm{H} \\ \text { P0 } & 13.35 \text { usec } \\ \text { P1 } & 13.35 \text { usec } \\ \text { P17 } & 2500.00 \text { usec }\end{array}$

$\begin{array}{lr}\text { P17 } & 2500.00 \text { us } \\ \text { PLW1 } & 16.00000000 \mathrm{~W} \\ \text { PLW10 } & 3.16840005 \mathrm{~W}\end{array}$

$=====$ GRADIENT CHANNEL $====$

GPNAM[1] SMSQ10.100

$\begin{array}{lr}\text { GPNAM[1] } & \text { SMSQ10.100 } \\ \text { GPZ1 } & 10.00 \% \\ \text { P16 } & 1000.00 \text { usec }\end{array}$

F1 - Acquisition parameters

TD - Acquisition parameters

$\begin{array}{lr}\text { TD } & 128 \mathrm{MHz} \\ \text { FIDRES } & 51.565657 \mathrm{~Hz}\end{array}$

$\begin{array}{lr}\mathrm{SW} & \text { 4.039 ppm } \\ \text { FnMODE } & \text { QF }\end{array}$

F2 - Processing parameters

$\begin{array}{lr}\text { SI } & \text { Processing parameters } \\ \text { SF } & 500.1300000 \mathrm{MHz}\end{array}$

$\begin{array}{ll}\text { WDW } & \\ \text { SSB } & \oplus \\ \text { LB } & \oplus \mathrm{Hz}\end{array}$

$\begin{array}{lll}\text { GB } & 0 & 1.40\end{array}$

F1 - Processing parameters

$\begin{array}{lr}\text { SI } & 1024 \\ \text { MC2 } & \text { QF }\end{array}$

$\begin{array}{lc}\text { MC2 } & \text { QF } \\ \text { SF } & 500.1300000 \\ \text { WDW } & \text { MHz } \\ \text { SSB } & \text { QSINE }\end{array}$

$\begin{array}{ll}\text { SSB } & \oplus \\ \text { LB } & \oplus \\ G B & \oplus\end{array}$ 
Current Data Parameters

NAME SSK-11-AMB-OALLYL-DISACCHARIDE-1H

EXPNO

PROCNO

$$
9
$$

F2 - Acquisition Parameters

Date_ 20181102

Tims

$$
3.42
$$

PROBHD $5 \mathrm{~mm}$ PABBO BB-

PULPROG zg30

TD

SOLVENT

NS 18

DS

$\begin{array}{lc}\text { SWH } & \mathbf{0} 223.685 \mathrm{~Hz}\end{array}$

FIDRES $\quad 0.151522 \mathrm{~Hz}$

AQ $\quad 3.2998593 \mathrm{sec}$

RG $\quad 161$

DW $\quad 60.800$ usec

DE $\quad 6.50$ usec

TE $296.1 \mathrm{~K}$

D1 $\quad 1.00000000 \mathrm{sec}$

TD0 1

$=======$ CHANNEL $\mathrm{f} 1 \mathrm{=}=======$

NUC1 1H

$\begin{array}{lc}\text { NUC1 } & 1 \mathrm{H} \\ \text { P1 } & 14.75 \text { usec } \\ \text { PL1 } & -1.00 \mathrm{~dB}\end{array}$

PL1W 10.56200695 W

SFO1 400.1324710 MH

F2 - Processing parameters

SI $\quad 32768$

SF $\quad 400.1300101 \mathrm{MHz}$

WDW EM

$\begin{array}{lll}\text { SSB } & 0 & \\ \text { LB } & & 0.30 \mathrm{~Hz}\end{array}$

$\begin{array}{lll}\text { GB } & \mathbf{0} & \mathbf{1 . 0 0}\end{array}$

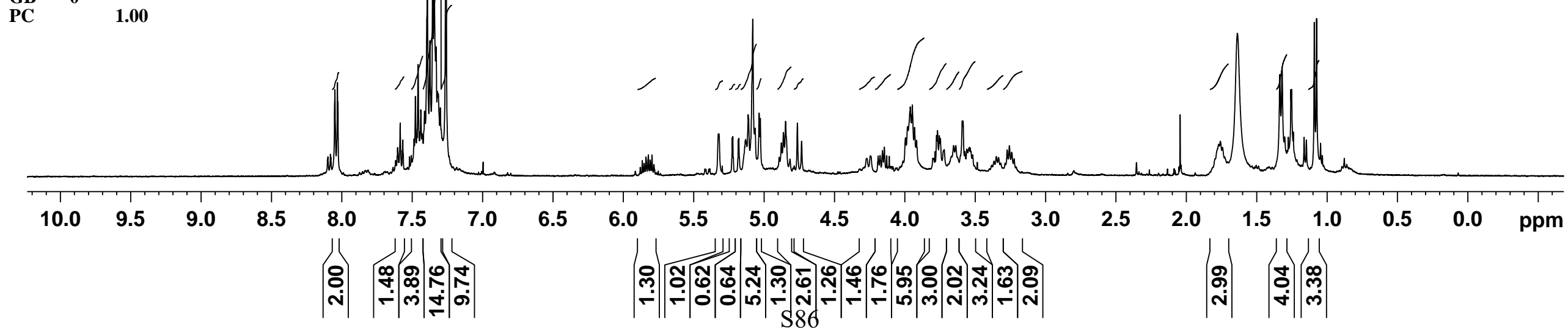




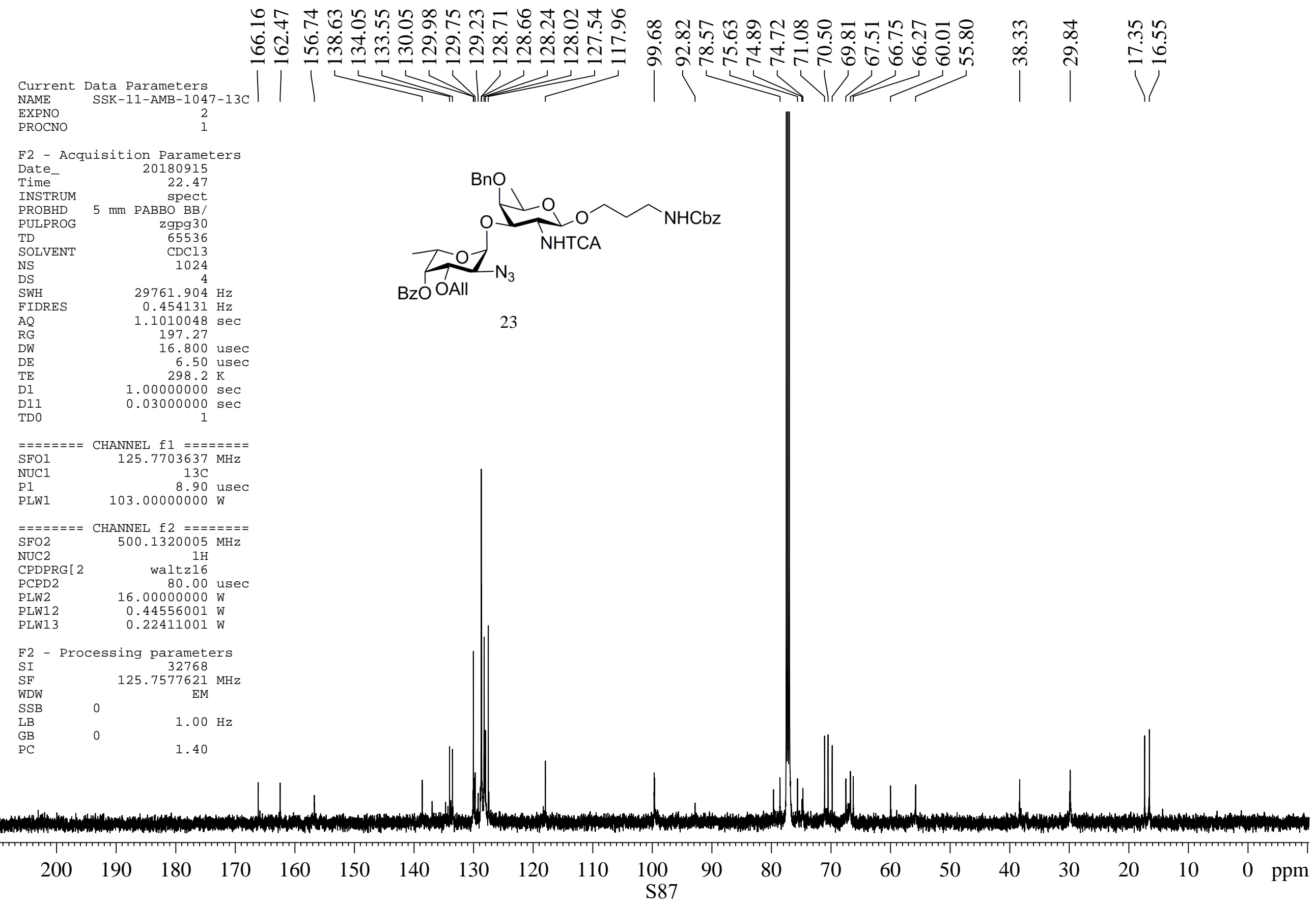


SSK - 11 - AMB - 1147 - DEPT

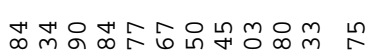

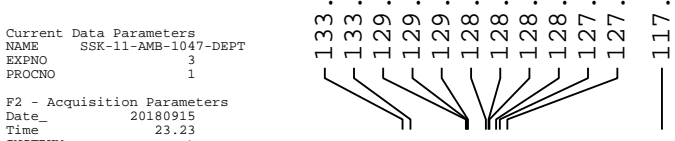

รัต

요

V

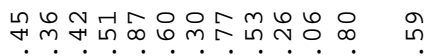

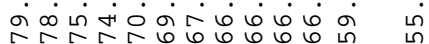

$111111 / 4$

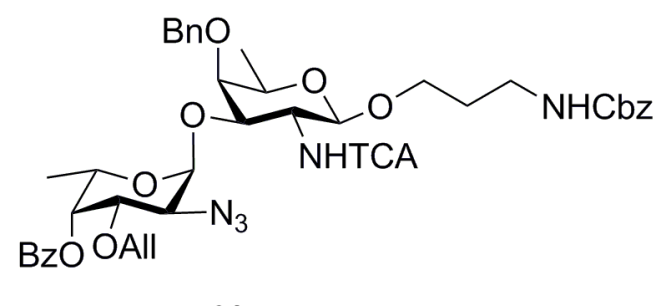

ก

mi

ते

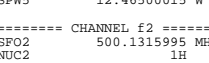

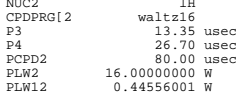

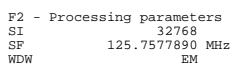

$\begin{array}{lll}\substack{\text { SISB } \\ \text { IIB } \\ \text { oB }} & 0 & 1.00 \mathrm{~Hz} \\ \text { PC } & 0 & 1.40\end{array}$

(1.40) 
$\mathrm{CH} 2$

SSK - 11 - AMB - OALLYL - DISACCHARIDE - COSY

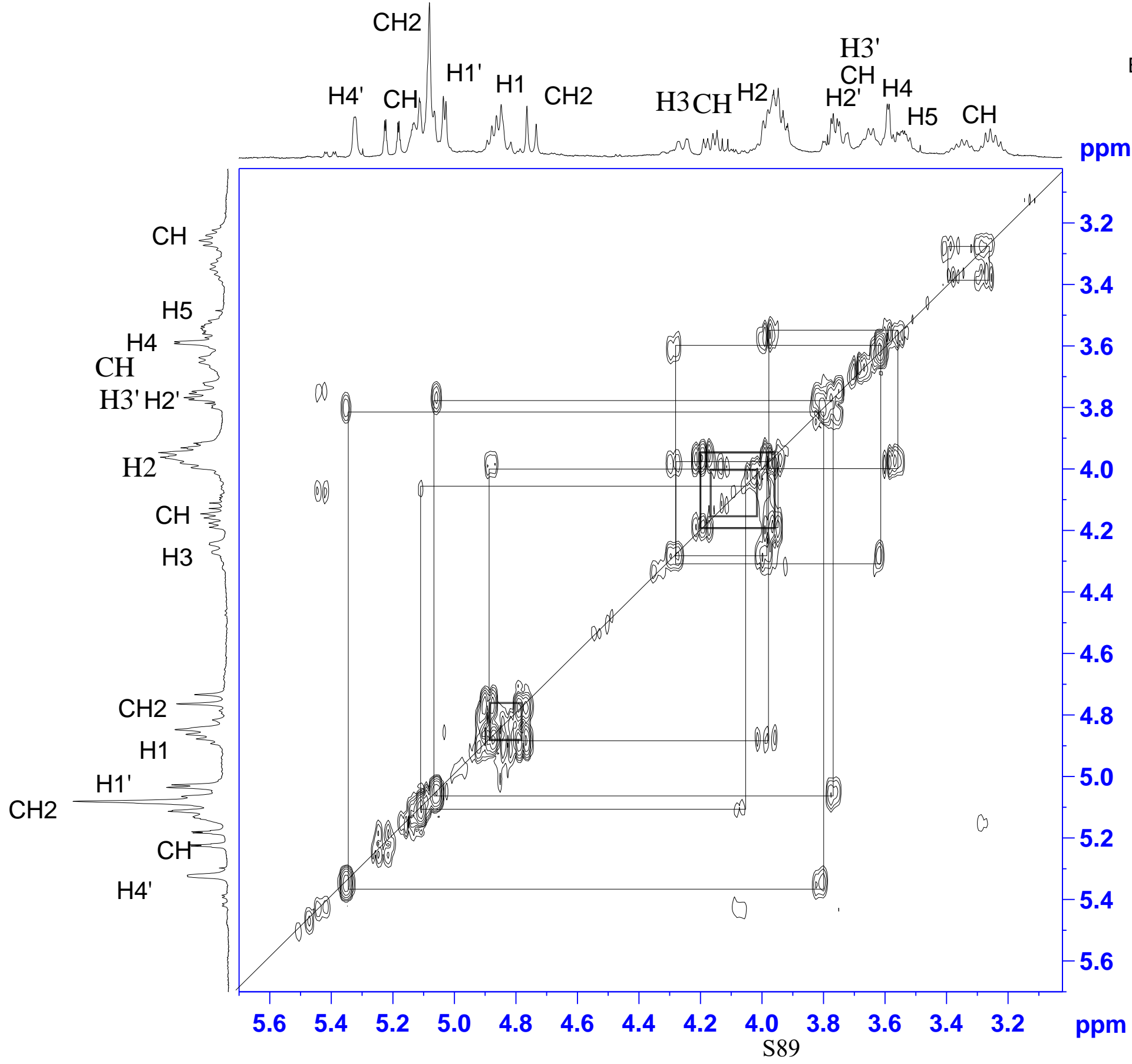

6
NHTCA ${ }_{B Z O O A l} N_{3}$

Current Data Parameters
NAEE SSK-11-AMB-OALYL-DISACCHARIDE-COSY EXPNO
PROCNO

E2-Actuisition paraters

F2 - Acquisition Parameter
Date

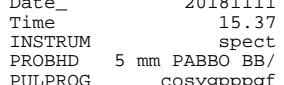

$\begin{array}{lr}\text { PULPROG } & \text { COSYgpppqf } \\ \text { TD } & 2048 \\ \text { SOLVENT } & \text { CDC13 } \\ \text { NS } & 8 \\ \text { NS } & 8\end{array}$

$\begin{array}{lr}\text { DS } & 0 \\ \text { SWH } & 4237.288 \mathrm{~Hz} \\ \text { FIDRES } & 2.069888 \mathrm{~Hz} \\ \text { AQ } & 0.241640 . \mathrm{sec}\end{array}$

$\begin{array}{lr}\text { FIDRES } & 2.068988 \mathrm{~Hz} \\ \text { AQ } & 0.2416640 \mathrm{sec} \\ \text { RG } & 61.42 \\ \text { DW } & 118.000 \text { use } \\ \text { W } & \end{array}$

$\begin{array}{cr}\text { DW } & 118.000 \text { use } \\ \text { DE } & 6.50 \text { use } \\ \text { DE } & 297.6 \mathrm{~K} \\ \text { D } & 0.00000300 \mathrm{sec} \\ \text { D1 } & 1.000000 \mathrm{sec} \\ \text { D1 } & 0.030000 \mathrm{sec}\end{array}$

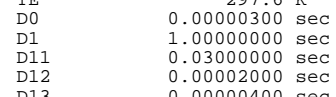

$\begin{array}{ll}\text { D13 } & 0.00000400 \mathrm{sec} \\ \text { D16 } & 0.00020000 \mathrm{sec} \\ \text { INO } & 0.0023000 \mathrm{sec}\end{array}$

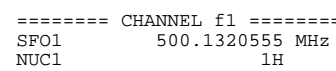

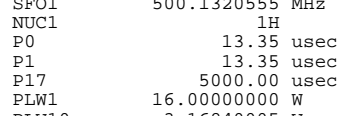

$\begin{array}{ll}\text { PLW1 } & 16.00000000 \mathrm{~W} \\ \text { PLW10 } & 3.16840005 \mathrm{~W}\end{array}$

$=====$ GRADIENT CHANNEL $===$
GPAM[1]
SMSQ10.1000
GPZ1
$10.00 \%$

$\begin{array}{lr}\text { GPNA1 } & \text { SMSQ10.100 } \\ \text { P16 } & 1000.00 \% \\ \text { use }\end{array}$

F1 - Acquisition parameters

$\begin{array}{lr}\text { TD } & 133 \\ \text { SFO1 } & 500.1321 \mathrm{MHz} \\ \text { FIDRES } & 63.718620 \\ \text { SW } & 8.472 \mathrm{ppm}\end{array}$

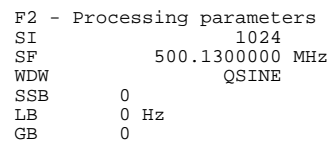

$\begin{array}{lll}\mathrm{GB} & 0 & 1.40\end{array}$

F1 - Processing parameters

$\begin{array}{cc}\text { SI } & 1024 \\ \text { MC2 } & \text { OF } \\ \text { SF } & 500.130000 \text { MHZ }\end{array}$

$\begin{array}{lll}\text { SF } & 500.13000000 \\ \text { WDW MHz } & \text { QSINE } \\ \text { SSB } & 0 & \text { QSI } \\ \text { LB } & 0 & \end{array}$ 
SSK - 11-AMB - $1065-1 \mathrm{H}$

Current Data Parameters

NAME SSK-11-AMB-1065-1H

EXPNO

7
1

F2 - Acquisition Parameters

$\begin{array}{lr}\text { Date_- } & 20180330 \\ \text { Time } & 16.58\end{array}$

INSTRUM spect

PROBHD $5 \mathrm{~mm}$ PABBO BB-

PULPROG

zg30

$\begin{array}{ll}\text { TD } & 54274 \\ \text { CDLVENT } & \text { CDCl3 }\end{array}$

NS 14

$\begin{array}{lr}\text { DS } & 0 \\ \text { SWH } & 8223.685 \mathrm{~Hz}\end{array}$

FIDRES $\quad 0.151522 \mathrm{~Hz}$

$\mathrm{AQ} \quad 3.2998593 \mathrm{sec}$

$\mathrm{RG}$

DW

DE 60.800 usec

6.50 use

TD॰

$1.00000000 \mathrm{sec}$

======== CHANNEL f1 ======== NUC1

P1

PL1

PL1W

14.75 usec

$-1.00 \mathrm{~dB}$

F2 - Processing parameters

SI $\quad 32768 \mathrm{MHz}$

WDW 400.1300101

SSB $\odot$

LB $\quad \odot$

$\odot .30 \mathrm{~Hz}$

1.00

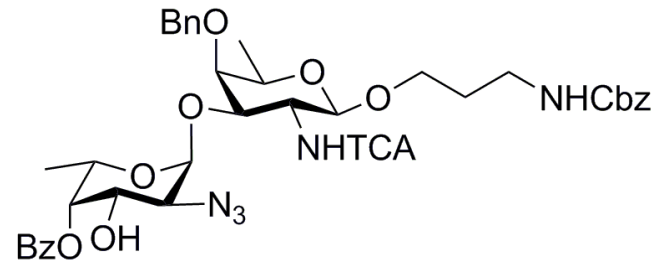

24
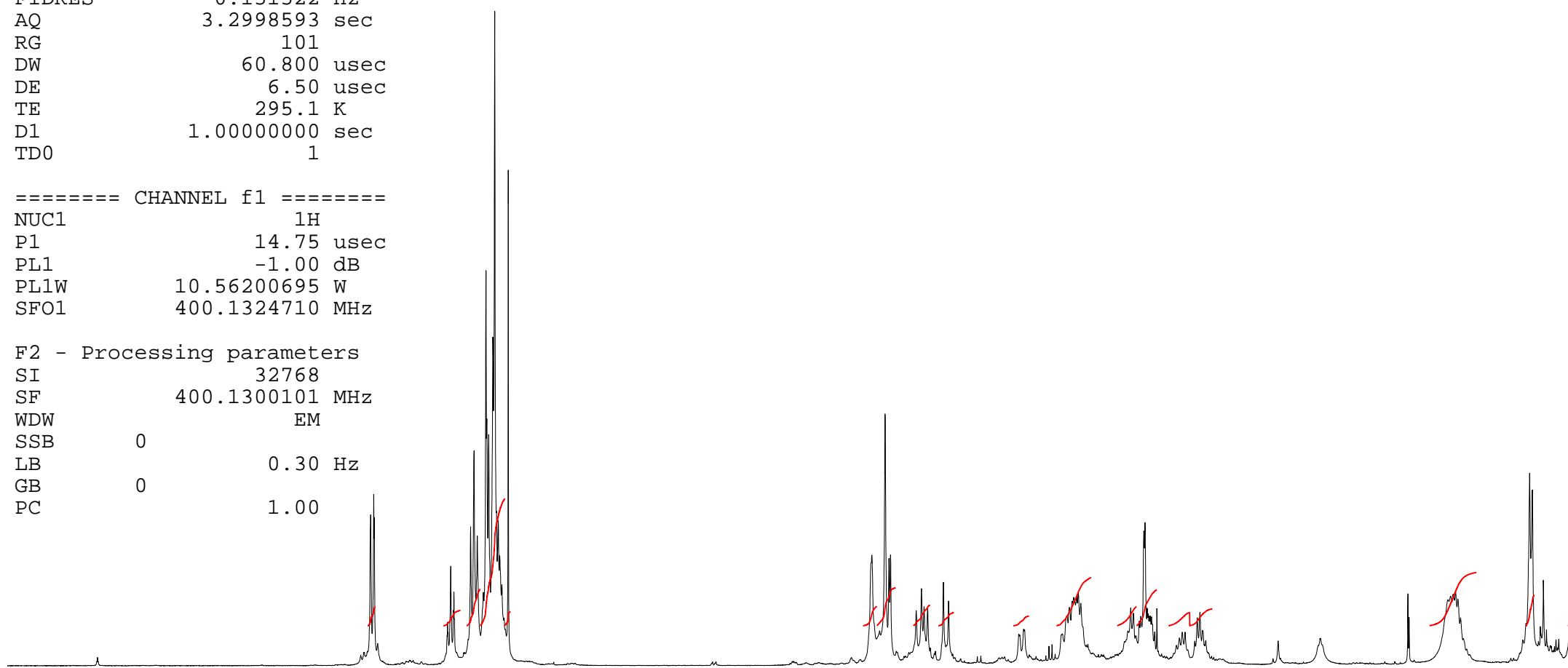

10

9

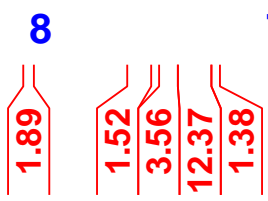

\begin{abstract}
7
\end{abstract}
6
3

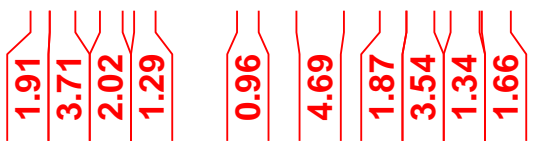

S91 
$\begin{array}{ll}\text { Current } & \text { Data Parameters } \\ \text { NAME } & \text { SSK-11-AMB-1065-13C }\end{array}$ EXPNO 2
1 F2 - Acquisition Parameters
Date 20180323

Dime 20180323
16.06

spect
PROBHD $5 \mathrm{~mm}$ PABBO BB-

PULPROG
TD

TD

SOLVENT

NS
DS
SWH

FIDRES $\quad 0.397364 \mathrm{~Hz}$

AQ $\quad 1.2582912 \mathrm{sec}$

RG

$\mathrm{DW}$

DE

D1

2050
19.200 use

6.50 usec

1. $000000000 \mathrm{Ke}$

$\odot .03000000 \mathrm{sec}$

$\begin{array}{lr}=======\text { CHANNEL } \mathrm{f} 1 \mathrm{n}====== \\ \text { NUC1 } & 13 \mathrm{C} \\ \text { P1 } & 8.50 \text { usec } \\ \text { PL1 } & -2.00 \mathrm{~dB}\end{array}$

PL1W

$56.53121948 \mathrm{~W}$

=-=-=-= CHANNEL f2 =-==-= CPDPRG [2 waltz16

$\begin{array}{ll}\text { NUC2 } & 1 \mathrm{H} \\ \text { PCPD2 } & 80.00 \text { usec }\end{array}$

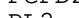

PL12

PL13

PL12W

PL13W
SF02

SI - Processing parameters

$\begin{array}{lr}\text { SI } & 32768 \\ \text { SF } & 100.6127529 \mathrm{MHz}\end{array}$

WDW $\quad 0 \quad$ EM

$\begin{array}{lll}\mathrm{LB} & 0 & 1.00 \mathrm{~Hz}\end{array}$

PC $\quad 1.40$

$-1.00 \mathrm{~dB}$

$13.69 \mathrm{~dB}$

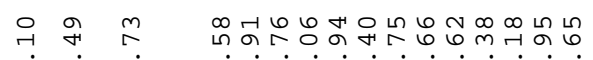

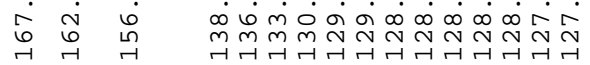

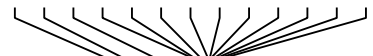

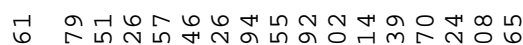

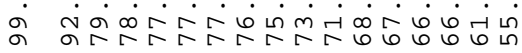

1111

® ำเ

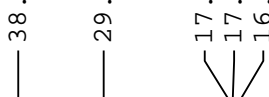

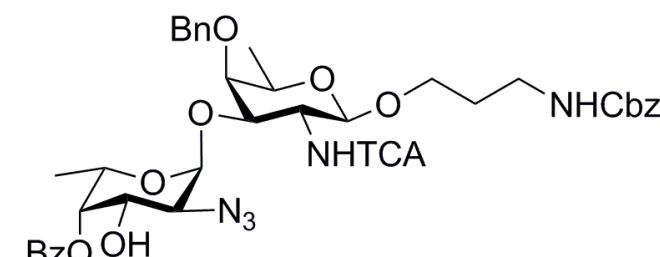

24

$\mathrm{BzOOH}$

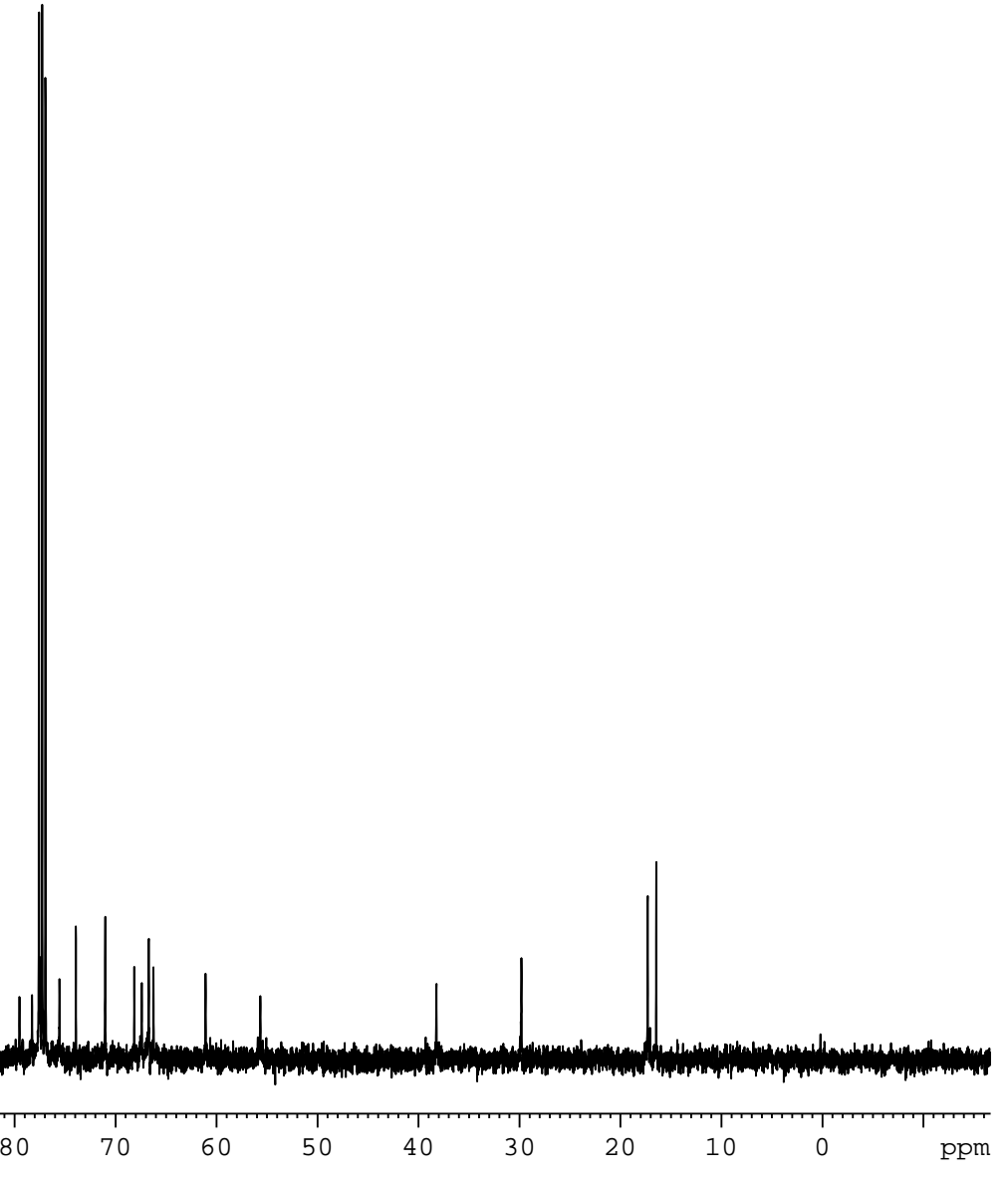


Current Data Parameters

NAME SSK-11-AMB-1065-DEPT EXPNO PROCNO

F2 - Acquisition Parameters

Date_r 20180323

$\begin{array}{lr}\text { Time } & 2018.14\end{array}$

spect
INSTRUM $5 \mathrm{~mm}$ PABBO BB-

PROBHD
PULPROG

TD

NS

SWH

FIDRES

RG

DW
DE
TE

CNST2

D1

D12 $\quad 0.00002000 \mathrm{sec}$

$=======$ CHANNEL $\mathrm{f}$

P2

PL1

PL1W

SF01

$======$
CPDPRG $[2$

f2 ======= waltz16

$\mathrm{CDCl} 3$
104
4

$24038.461 \mathrm{~Hz}$

(36

0.800 use

6.50
$297.2 \mathrm{~K}$

145.0000000

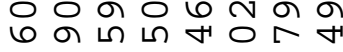

$00 \infty 0000$

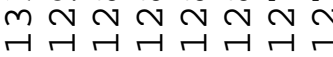

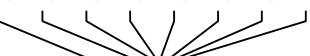

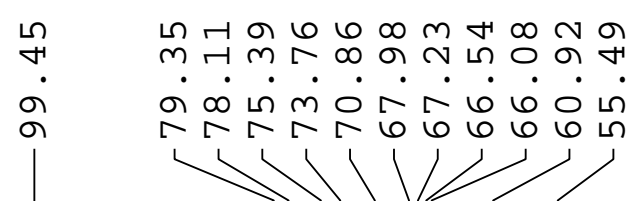

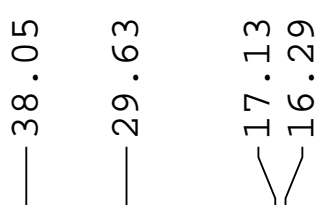

PCPD2

PL2

PL12

PL2W

PL12W
SF02

F2 - Processing parameters

$\begin{array}{lr}\text { SI } & 32768 \\ \text { SF } & 100.6127690 \mathrm{MHz}\end{array}$

WDW

$\begin{array}{lll}\text { SSB } & 0 & 3.00 \mathrm{~Hz}\end{array}$

$\mathrm{GB}$

190180 
SSK-11-AMB - 4-OBZ - 3-OAC-Donor - 1H

Current Data Parameters
NAME
SSK-11-AMB-4-OBZ-3-OAC-DONOR-1H

EXPNO

F2- Acquisition Parameters

Date
Time
INSTRUM

$\begin{array}{lr}\text { Time } & 0.30 \\ \text { INSTRUM } & \text { spect } \\ \text { PROBHD } & 5 \mathrm{~mm} \text { PABBO BB/ } \\ \text { PULPROG } & \mathrm{gBg} 3 \\ \text { TLPROG } & 65536\end{array}$

SOLVENT

$\begin{array}{lr}\text { NS } & 9 \\ \text { DS } & 0 \\ \text { SWH } & 10000.000\end{array}$

$\begin{array}{ll}\text { FIDRES } & 0.1525808 \mathrm{~Hz} \\ \mathrm{AQ} & 3.2767999 \mathrm{sec}\end{array}$

$\begin{array}{lr}R G & 119.07 \\ D W & 50.000 \text { usec } \\ D E & 6.50 \text { usec }\end{array}$

$\begin{array}{lr}\text { TE } & 297.1 \mathrm{~K} \\ \text { D1 } & 1.00000000 \mathrm{sec} \\ \text { TDO } & 1\end{array}$

$========$ CHANNEL
SF $11=======$
SF01

$\begin{array}{lr}\text { SF01 } & 500.1330885 \mathrm{MHz} \\ \text { NUC1 } & 1 \mathrm{H} \\ \text { P1 } & 13.35 \mathrm{usec} \\ \text { PLW1 } & 16.00000000 \mathrm{~W}\end{array}$

F2 - Processing parameters

$\begin{array}{lr}\text { SI } & 65536 \\ \text { SF } & 500.1300133 \\ \text { MHz }\end{array}$

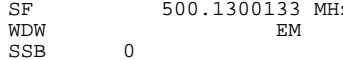

$\begin{array}{lll}\text { SSB } & 0 & 0.30 \mathrm{~Hz} \\ \mathrm{LB} & 0 & \end{array}$

$\begin{array}{lll}\mathrm{GB} & 0 & 1.00\end{array}$

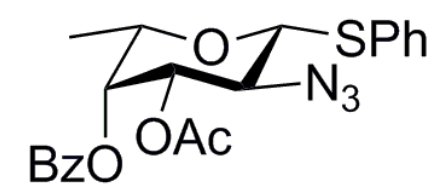

$7 b$

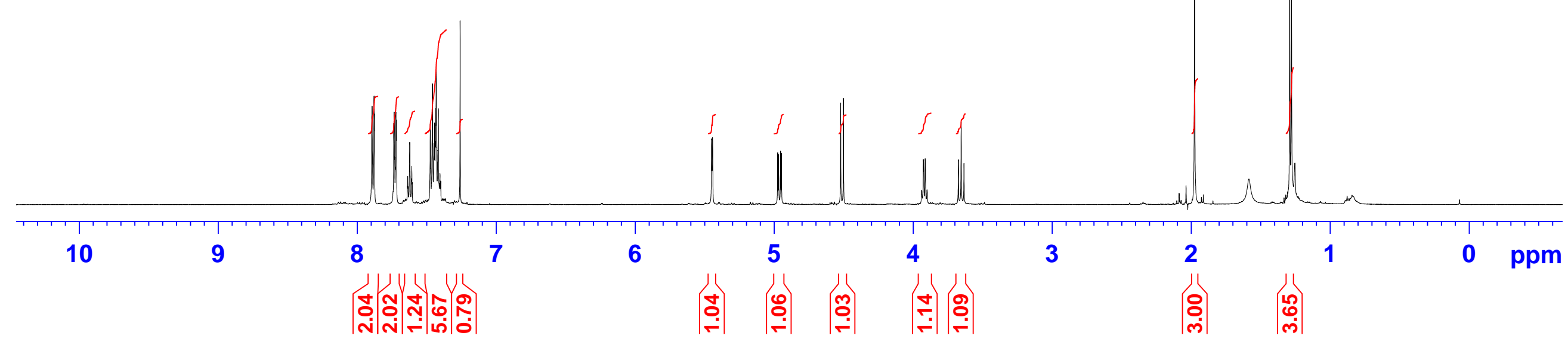


SSK-11-AMB - 4-OBZ-3-OAC-DONOR-13C

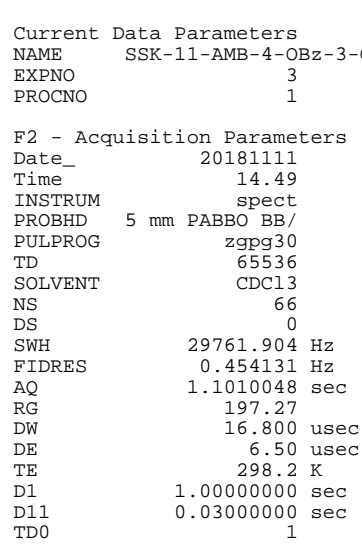

$$
\begin{aligned}
& \begin{array}{l}
======= \\
\text { CHANNEL } \mathrm{f} 1======= \\
125.7703637 \mathrm{MHZ} \\
\text { SFO1 } \\
\text { NUC1 }
\end{array} \\
& \begin{array}{lr}
\text { NUC1 } & 13 \mathrm{C} \\
\text { P1 } & 8.90 \text { usec } \\
\text { PLW1 } & 103.00000000 \mathrm{~W}
\end{array}
\end{aligned}
$$

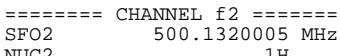

$$
\begin{aligned}
& \begin{array}{lr}
\text { NUC2 } & 500.1320005 \\
\text { CPDPRG [2 } & 1 \mathrm{H} \\
\text { PCPD2 } & \text { waltz16 }
\end{array} \\
& \begin{array}{lc}
\text { CPDPRG [2 } & \text { waltz16 } \\
\text { PCD2 } & 80.00 \text { usec } \\
\text { PLW2 } & 16.00000000 \mathrm{~W}
\end{array} \\
& \begin{array}{lr}
\text { PLW12 } & 0.44556001 \mathrm{~W} \\
\text { PLW13 } & 0.22411001 \mathrm{~W}
\end{array} \\
& \text { F2 - Processing parameters } \\
& \begin{array}{lr}
\text { SI } & 32768 \\
\text { SF } & 125.7577602 \mathrm{MHz} \\
\text { WDW } &
\end{array} \\
& \begin{array}{lll}
\text { WDW } & & \text { EM } \\
\text { SSB } & 0 & 1.00 \mathrm{~Hz}
\end{array} \\
& \begin{array}{lll}
\mathrm{LB} & 0 & 1.00 \mathrm{~Hz} \\
\mathrm{~GB} & 0 & 1.40
\end{array}
\end{aligned}
$$
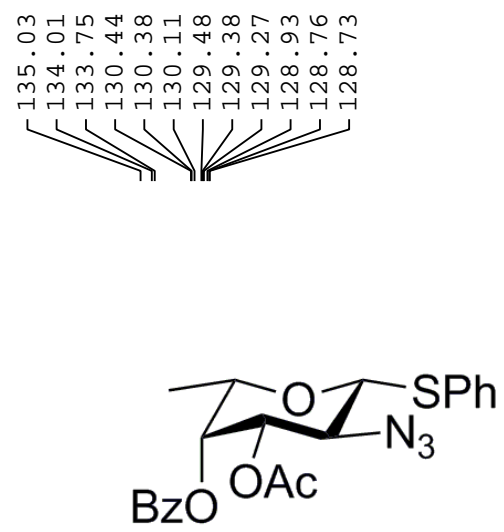

$7 b$

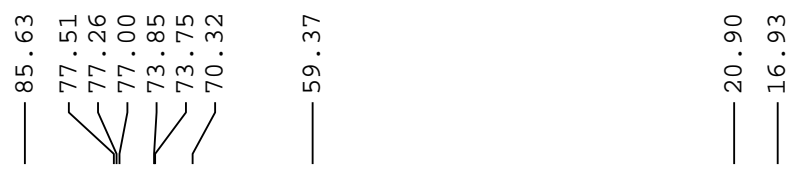

照

$\stackrel{2}{2}$

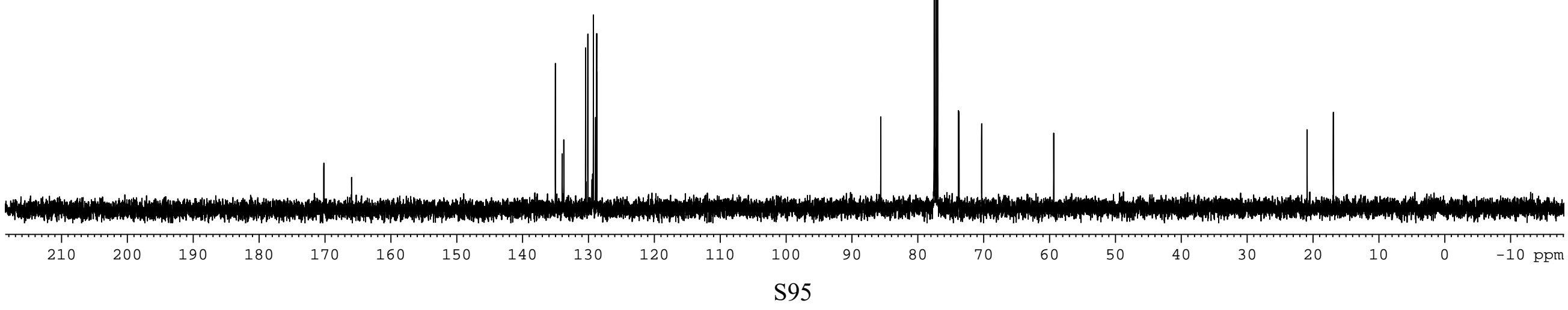


SSK - 11 - AMB - 4 - OBZ - 3 - OAC - DONOR - COSY

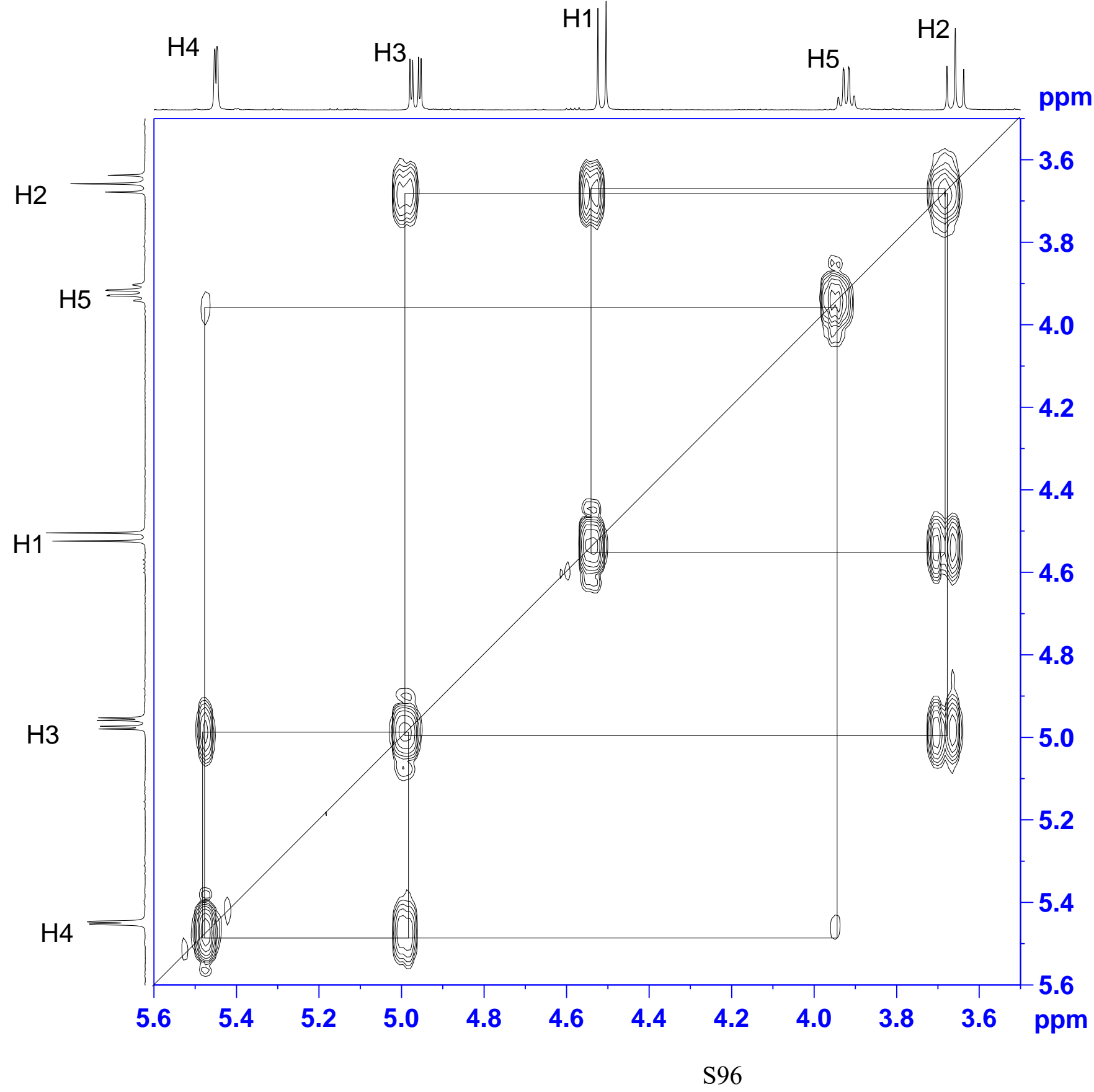

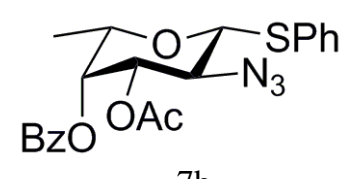

$7 b$

Current Data Parameters
NAME SSK-11-AMB-4-OBZ-3-OAC-DONOR-COSY EXPNO

F2 - Acquisition Parameters

D2 -

Time-
INSTRUM

$\begin{aligned} \text { PROBHD } & \text { mm PABBO BB/ } \\ \text { PLPROG } & \text { cosygpppaf }\end{aligned}$

SOLVENT

SOLVENT
NS
DS
SWH

$\begin{array}{lr}\text { DS } & 8 \\ \text { SWH } & 0 \\ \text { FIDRES } & 2545.455 \mathrm{~Hz}\end{array}$

$\begin{array}{lr} & 4545.455 \mathrm{~Hz} \\ \text { FIDRES } & 2.219460 \mathrm{~Hz} \\ \text { AQ } & 0.2252800 \mathrm{sec} \\ \text { RG } & 61.42\end{array}$

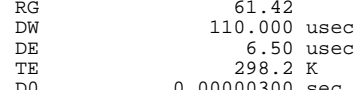

$\begin{array}{ll}\text { TE } & 298.2 \mathrm{~K} \\ \text { TE } & 0.00000300 \\ \text { De } & \text { sec } \\ \text { D1 } & 2.00000000 \text { sec } \\ \text { D11 } & 0.03000000 \text { sec } \\ \text { D1 } & 0.0002000\end{array}$

$\begin{array}{ll}\text { D1 } & 0.0000000 \text { sec } \\ \text { D11 } & 0.0300000 \text { sec } \\ \text { D12 } & 0.00002000 \text { sec } \\ \text { D13 } & 0.00000400 \text { sec } \\ \text { Dec } & \text { Sec }\end{array}$

$\begin{array}{ll}\text { D16 } & 0.00020000 \text { sec } \\ \text { INO } & 0.00022000 \text { sec }\end{array}$

$=======$
SF01
CHANNEL $\mathrm{f} 1========$
$500.1320923 \mathrm{MHz}$

$\begin{array}{lr}\text { NUC1 } & 13.35 \mathrm{usec} \\ \text { P0 } & 13.35 \text { usec } \\ \text { P1 } & 5000.00 \text { usec } \\ \text { P17 } & 16.000900 \text { W }\end{array}$

$\begin{array}{ll}\text { PLW1 } & 16.00000000 \mathrm{~W} \\ \text { PLW10 } & 3.16840005 \mathrm{~W}\end{array}$

$=====$ GRADIENT CHANNEL $===$

$\begin{array}{ll}\text { GRP1 } & \text { PI1 } \\ \text { P16 } & 1000.00 \%\end{array}$

F1 - Acquisition parameters

SF01 $\quad 500.1321 \mathrm{MHZ}$

FWW $\quad 75.755756 \mathrm{~Hz}$

$\begin{array}{lc}\text { F2 } & \text { - Processing parameters } \\ \text { SI } & 1024 \\ \text { SF } & 500.1300000 \\ \text { SDW } & \text { QSINE }\end{array}$

WDW 0

$\begin{array}{lll}\mathrm{SB} & \ominus \\ \mathrm{LB} & \odot \mathrm{Hz} \\ \mathrm{GB} & \odot & \\ \mathrm{PC} & & \end{array}$

F1 - Processing parameters

$\begin{array}{lc}\text { SI } & 1024 \\ \text { MC2 } & \text { PF } \\ \text { SF } & 500.1300000 \\ \text { SH MHZ }\end{array}$

$\begin{array}{ll}\text { WDW } & \\ \text { SSB } & 0 \\ \text { LB } & 0 \\ \text { GB } & 0\end{array}$ 
Current Data Parameters

NAME SSK-24-DR-183-1H

EXPNO

4
1

F2 - Acquisition Parameters

$\begin{array}{lr}\text { Date_ } & 20181102 \\ \text { Time } & 2.41\end{array}$

INSTRUM

PROBHD

PULPROG

$5 \mathrm{~mm}$ РАВBO BB-

Zg30

$\begin{array}{ll}\text { TD } & 54274 \\ \text { SOLVENT } & \text { CDC13 }\end{array}$

NS $r$

FIDRES

AQ

RQ

DW

DE

D1

TD $\odot$

$=======$ CHANNEL f1 =======

NUC1

P1

PL1

PL1W

SF01

F2 - Processing parameters

SI $\quad 32768$

SF $\quad 400.1300100 \mathrm{MHz}$

WDW EM

$\begin{array}{lll}\text { SSB } & \odot & 0.30 \mathrm{~Hz}\end{array}$

GB

PC

1.00

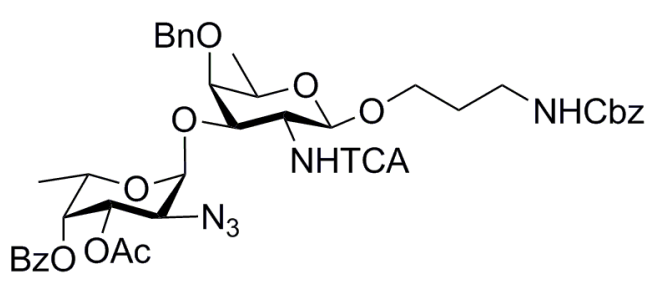

25

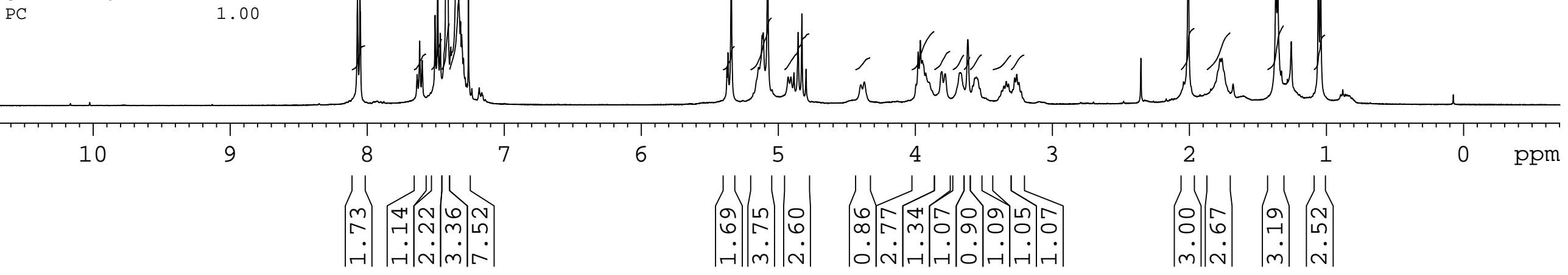


Current Data Parameters

NAME SSK-24-DR-183-13C

EXPNO

1

ameters

D2 - Acquisition Parameter

Time

20181102
2.44

INSTRUM $5 \mathrm{mpect}$

PULPROG $5 \mathrm{~mm}$ PApg 30

TD $\quad 65536$

$\begin{array}{lr}\text { SOLVENT } & \text { CDCl3 } \\ \text { NS } & 211 \\ \text { DS } & 0\end{array}$

SWH 26041.666 Hz

FIDRES $\quad 0.397364 \mathrm{~Hz}$

$\mathrm{AQ} \quad 1.2582912 \mathrm{sec}$

RG 1030

$\begin{array}{lr}\text { DW } & 19.200 \text { usec } \\ \text { DE } & 6.50 \text { usec }\end{array}$

TE $297.0 \mathrm{~K}$

$\begin{array}{ll}\text { D1 } & 1.000 \odot \odot \odot \odot \odot ~ \\ \text { D11 } & 0.0300000 \% \mathrm{sec}\end{array}$

$\begin{array}{rr}\text { D11 } & 0.0300000 \odot \\ \text { TD0 } & 1\end{array}$

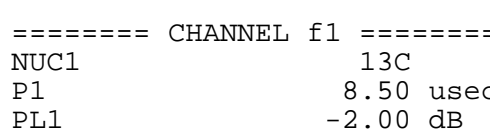

PL1W 56.53121948 W

SF01 100.6238364 MHz

$=======$ CHANNEL f2 $========$

CPDPRG [2 waltz16

$\begin{array}{lr}\text { NUC2 } & 1 \mathrm{H} \\ \text { PCPD2 } & 80.00 \text { usec } \\ \text { PL2 } & -1.00 \mathrm{~dB}\end{array}$

PL12

PL13

PL2W

PL12W

PL13W

$-1.00 \mathrm{~d}$
$13.69 \mathrm{~d}$

$14.50 \mathrm{~dB}$

$10.56200695 \mathrm{~W}$

$0.35871249 \mathrm{~W}$

$0.29767781 \mathrm{~W}$

$400.1316005 \mathrm{MHz}$

F2 - Processing parameters

SI 32768

SF $\quad 100.6127491 \mathrm{MHz}$

WDW EM

$\begin{array}{lll}\text { SSB } & 0 & 1.00 \mathrm{~Hz}\end{array}$

$\begin{array}{lll}\text { GB } & \odot & 1.00\end{array}$

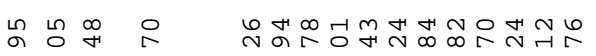

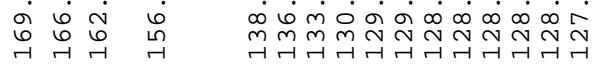

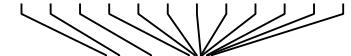

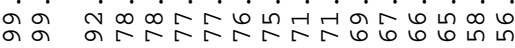

V W

m $\quad \infty \quad \infty \quad \infty \quad \infty$

m $\quad \dot{\sim} \quad \dot{i} \tilde{r}$

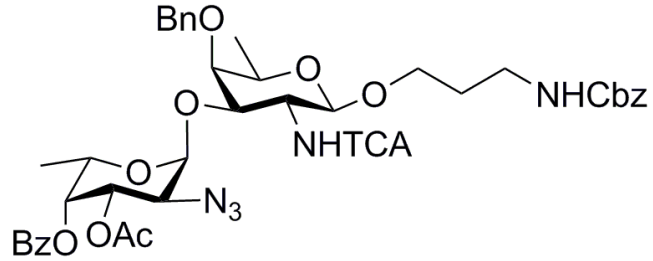

25 
SSK - 24 - DR - 183 - DEPT

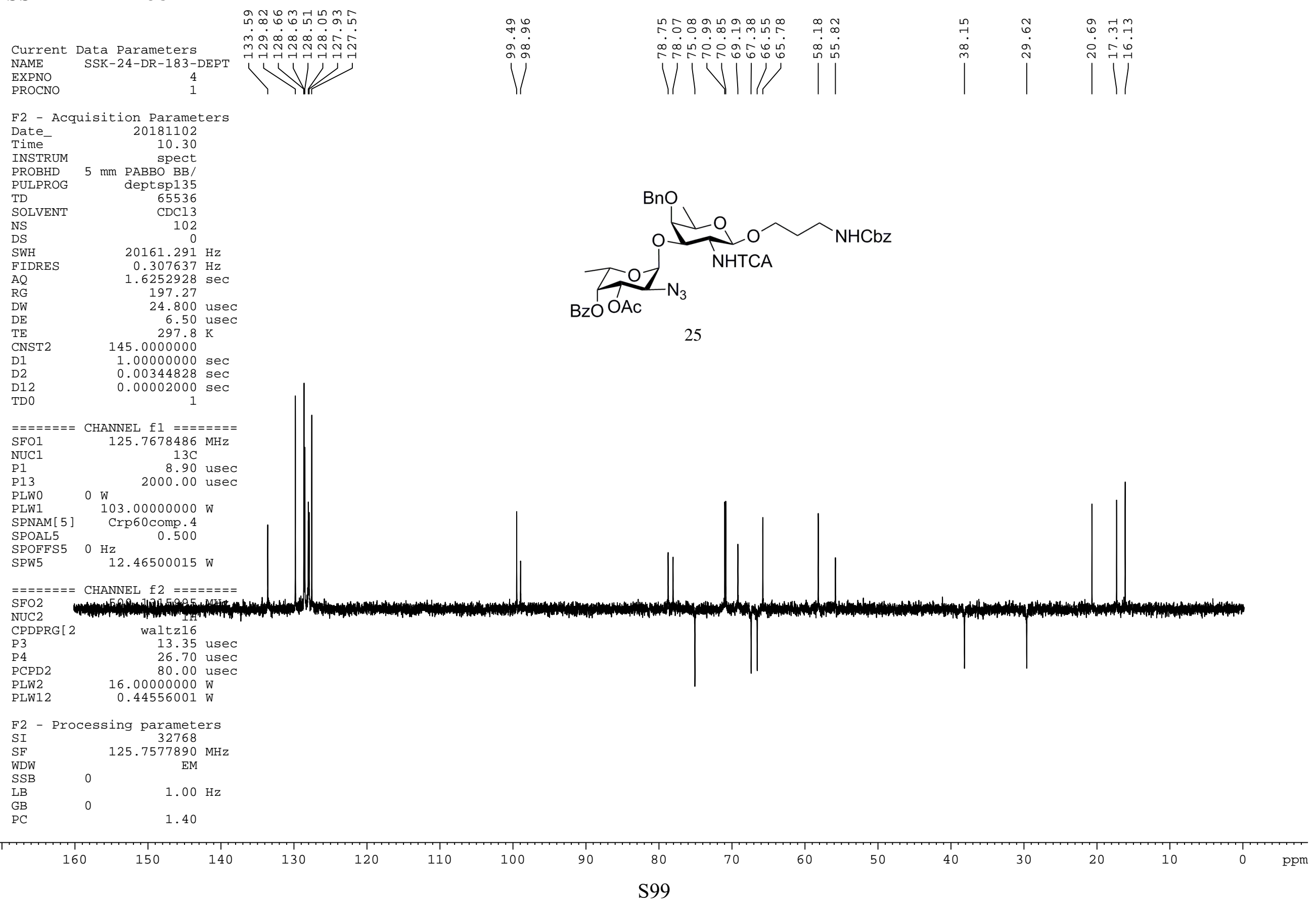


SSK-24-DR-183-COSY

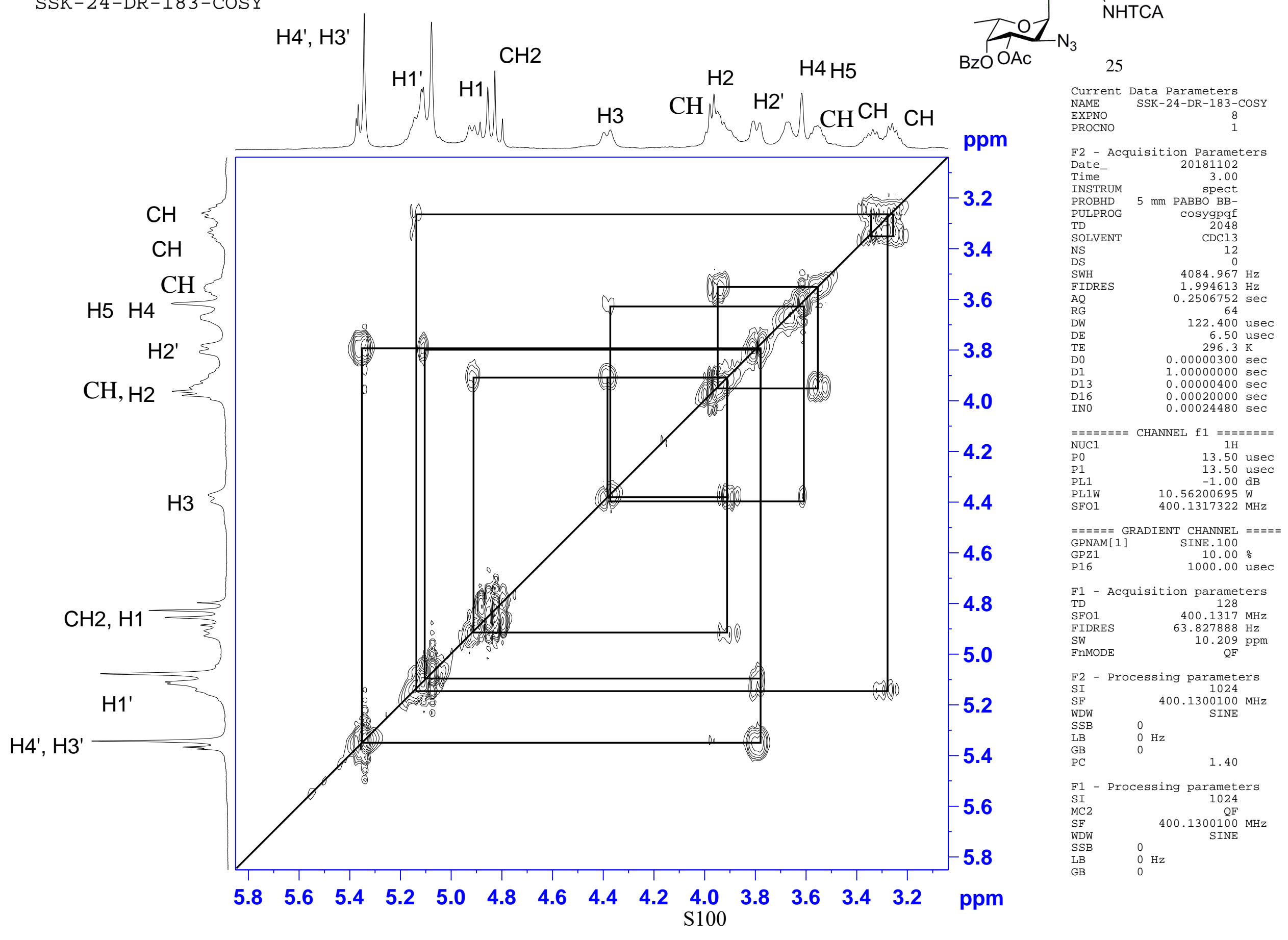


SK-24-DR-183-HSQC

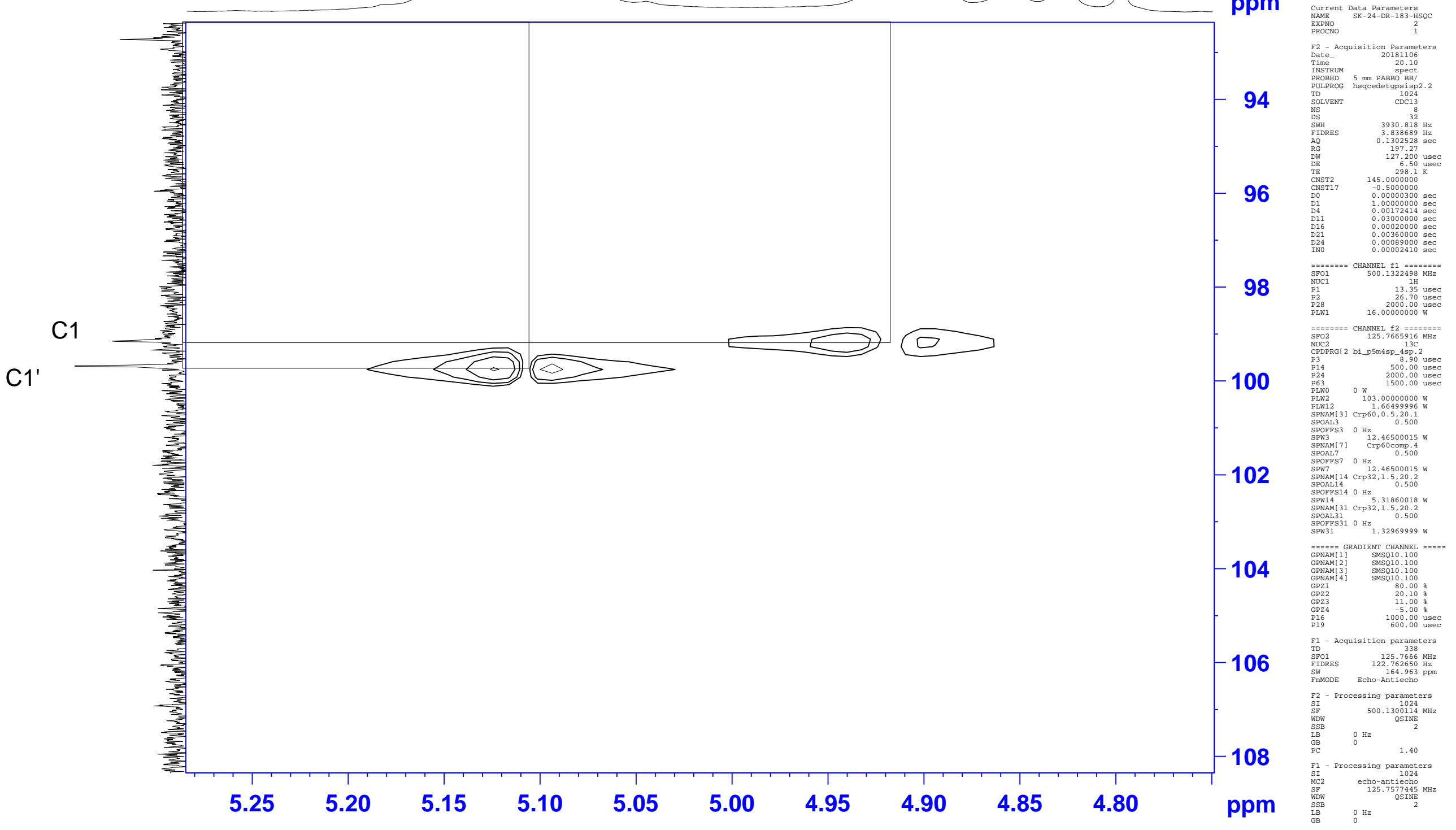


SSK - 11 - AMB - $1189-1 \mathrm{H}$

Current Data Parameters

NAME SSK-11-AMB-1189-1H

EXPNO

1

F2 - Acquisition Parameters

Date_ 20181002

INSTRUM

PROBHD

PULPROG

$5 \mathrm{~mm}$ PABBO BB-

TD

NS

SWH

10

SWH 8223.685 Hz

AQ $\quad 3.2998593 \mathrm{sec}$

$\mathrm{RG}$

DW

DE

TE

60.800 usec

6.50 usec

$1.00000000 \mathrm{sec}$

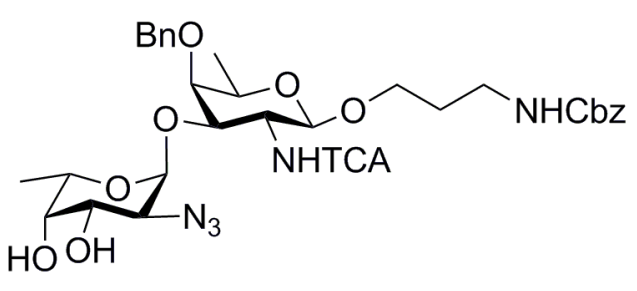

$=======$ CHANNEL $f 1$ ======== NUC1 NUC1

P1

PL1

PL1W

14.75 usec

F2 - Processing parameters

SI 32768

SF $\quad 400.1300101 \mathrm{MHz}$

WDW $\quad \odot$

$\begin{array}{ll}\text { LB } & \\ \text { GB } & 0\end{array}$

PC

$\odot .30 \mathrm{~Hz}$

1.00

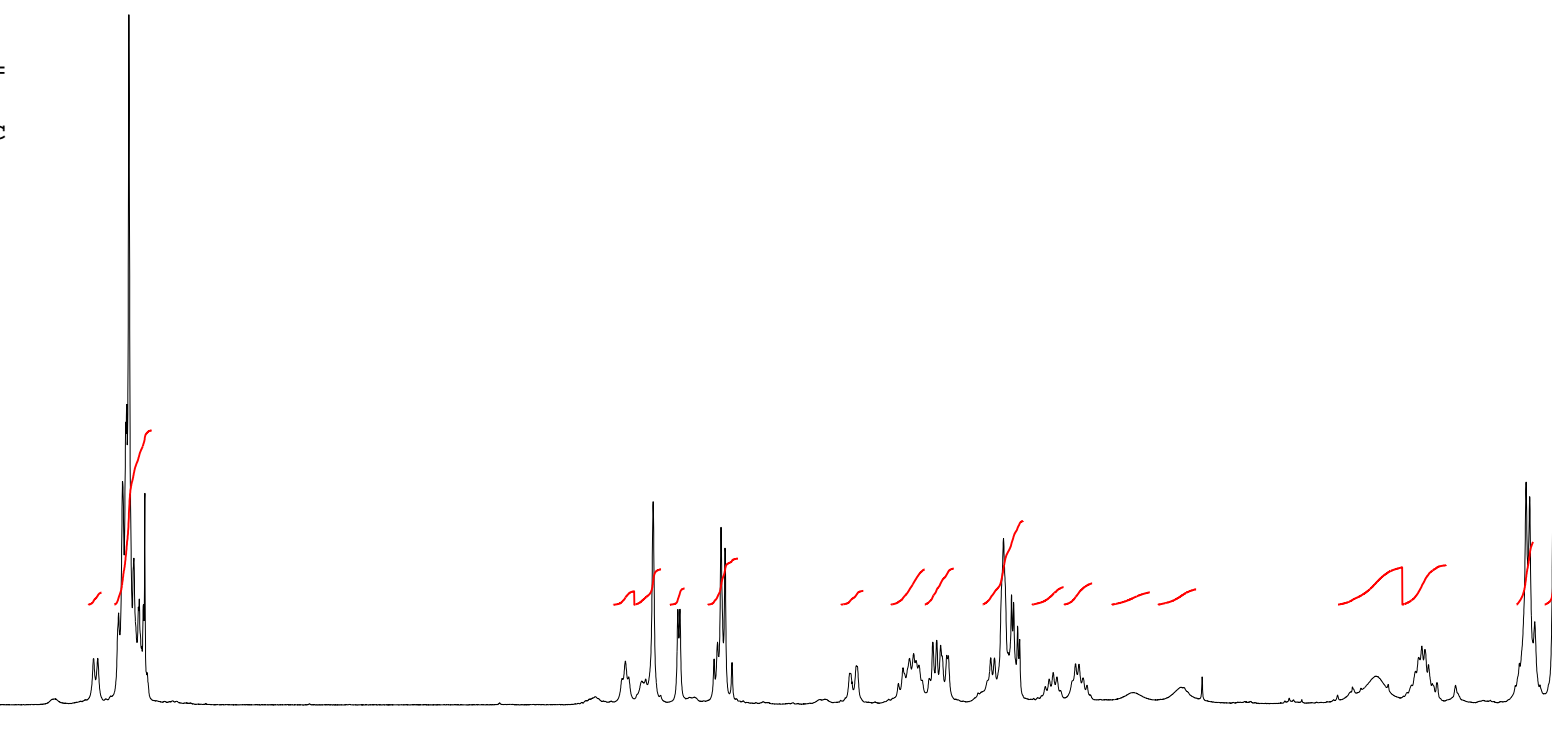

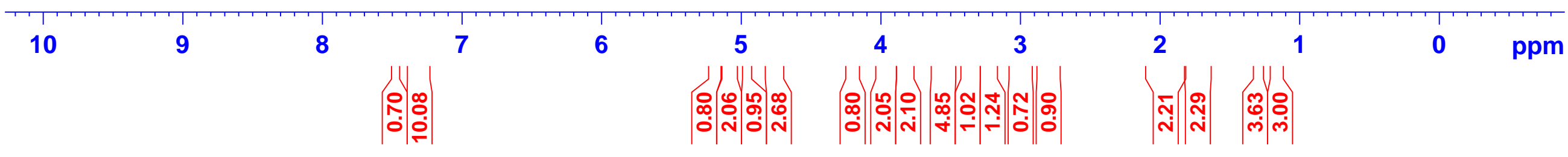


Current Data Parameters

NAME SSK-11-AMB-1189-13C

EXPNO

PROCNO

2

F2 - Acquisition Parameters

Date

20181002

INSTRUM

PROBHD

PULPROG

SOLVENT

NS

DS

SWH

FIDRES

AQ

DW

DE

D1

D1
D11
TD0

$=====$

NUC1

$\mathrm{P} 1$

PL1

PL1W

$5 \mathrm{~mm}$ РABBO BB-

Zgpg30
65536

$\mathrm{CDCl3}$ 255

$26041.666 \mathrm{~Hz}$

$1.2582912 \mathrm{sec}$$$
2050
$$

19.200 usec $\begin{aligned} & 6.50 \text { US } \\ & 296.7 \mathrm{~K}\end{aligned}$

$1.000000000 \mathrm{sec}$

$0.03000000 \mathrm{sec}$

CHANNEL f1 ======== $13 \mathrm{C}$

8.50 usec

$56.53121008 \mathrm{~dB}$

$56.53121948 \mathrm{~W}$

$100.6238364 \mathrm{MHz}$

$=======$ CHANNEL $\mathrm{f} 2$ ========
CPDPRG $[2$ waltz16

NUC2 2

PCPD2

PL2

PL12

PL13

PL2W

PL12W

PL13W

$13.69 \mathrm{~dB}$
$14.50 \mathrm{~dB}$

$10.56200695 \mathrm{~W}$

$0.35871249 \mathrm{~W}$

- Processing parameters

$\begin{array}{lr}\text { SI } & 32768 \\ \text { SF } & 100.6127478 \mathrm{MHz}\end{array}$

WDW $\quad 100.6127478$ MM

$\begin{array}{lll}\text { SSB } & 0 & 1.00 \mathrm{~Hz}\end{array}$

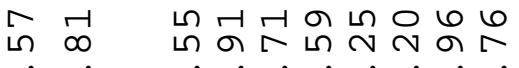

$\sim 0$ ம

$\stackrel{\sim}{\mathrm{m}}$

0 ก

HभH

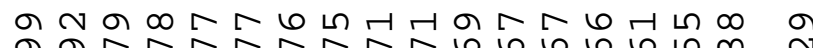

नि
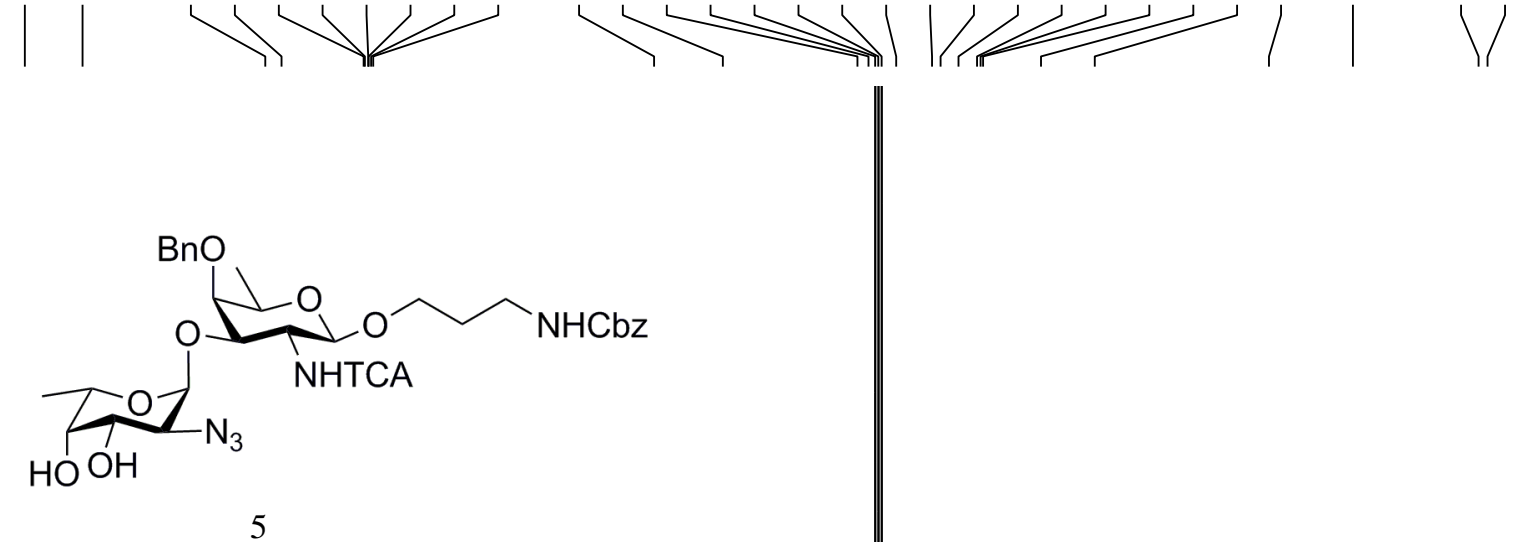

100 
$\begin{array}{ll}\text { Current } & \text { Data Parameters } \\ \text { NAME } & \text { SSK-11-AMB-1189-DEPT }\end{array}$

EXPNO

3
1

ROCNO

F2 - Acquisition Parameters

Date

$$
\begin{array}{r}
20181002 \\
8.32
\end{array}
$$

Time

INSTRUM

PULPROG

$5 \mathrm{~mm}$ РABBO BB-

dept 135

SOLVENT

NS

DS

SWH

AQ

RG

DE

TE

CNST2

D1

D12

65536
$\operatorname{CDC} 13$

75

$24038.461 \mathrm{~Hz}$

$0.366798 \mathrm{~Hz}$

$1.3631488 \mathrm{sec}$

20.800 usec 6.50 usec

145.0000000

1. $00000000 \mathrm{sec}$ $0.00344828 \mathrm{sec}$

$\odot .0000200 \odot \mathrm{sec}$

$=======$ CHANNEL $\mathrm{f} 1========$
NUC1 $\begin{array}{lr}\text { NUC1 } & 13 \mathrm{C} \\ \text { P1 } & 8.50 \text { usec } \\ \text { P2 } & 17.00 \text { usec }\end{array}$

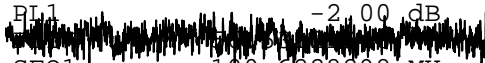

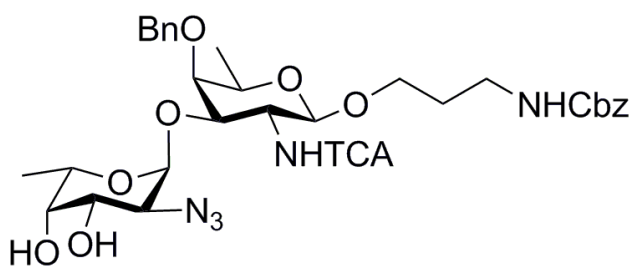

$========$ CHANNEL f2 ======== CPDPRG [2 waltz16

$\begin{array}{lr}\text { NUC2 } & 1 \mathrm{H} \\ \text { P3 } & 14.75 \mathrm{usec} \\ \text { P4 } & 29.50 \mathrm{usec} \\ \text { PCPD2 } & 80.00 \mathrm{usec} \\ \text { PL2 } & -1.00 \mathrm{~dB} \\ \text { PL12 } & 13.69 \mathrm{~dB} \\ \text { PL2W } & 10.56200695 \mathrm{~W} \\ \text { PL12W } & 0.35871249 \mathrm{~W} \\ \text { SF02 } & 400.1316005 \mathrm{MHz}\end{array}$

SF02 $\quad 0.35871249 \mathrm{~W}$

F2 - Processing parameters

SI - 32768

$\begin{array}{ll}\text { SF } & 100.6127690 \mathrm{MHz}\end{array}$

$\begin{array}{lll}\text { WSB } & 0 & \\ \text { SB } & & \\ \text { L } & & \end{array}$

$\begin{array}{lll}\mathrm{LB} & 3.00 \mathrm{~Hz} \\ \mathrm{~GB} & 0 & 1.40\end{array}$

$\begin{array}{llll}210 & 200 & 190 & 180\end{array}$

150

80

$7 \odot$


SSK - 11-AMB - $1193-3-0$-TRISACCHARIDE - 1H

Current Data Parameters
NAME SSK-11-AMB-1193-3-0-TRISACCHARIDE-1H

$$
\begin{aligned}
& \text { SSK-11-AMB-1193-3-0-TRISACCHARIDE-1H } \\
& \begin{array}{l}
\text { EXPNO } \\
\text { PROCNO }
\end{array}
\end{aligned}
$$

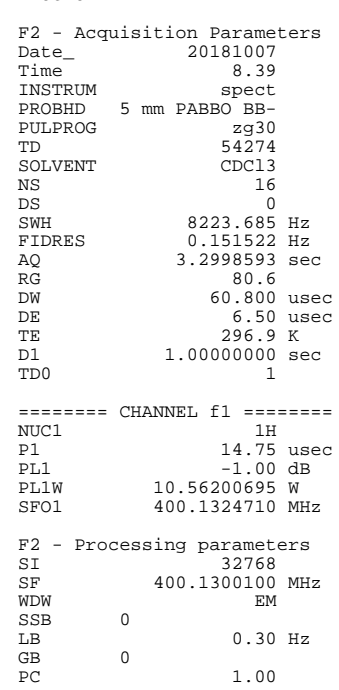
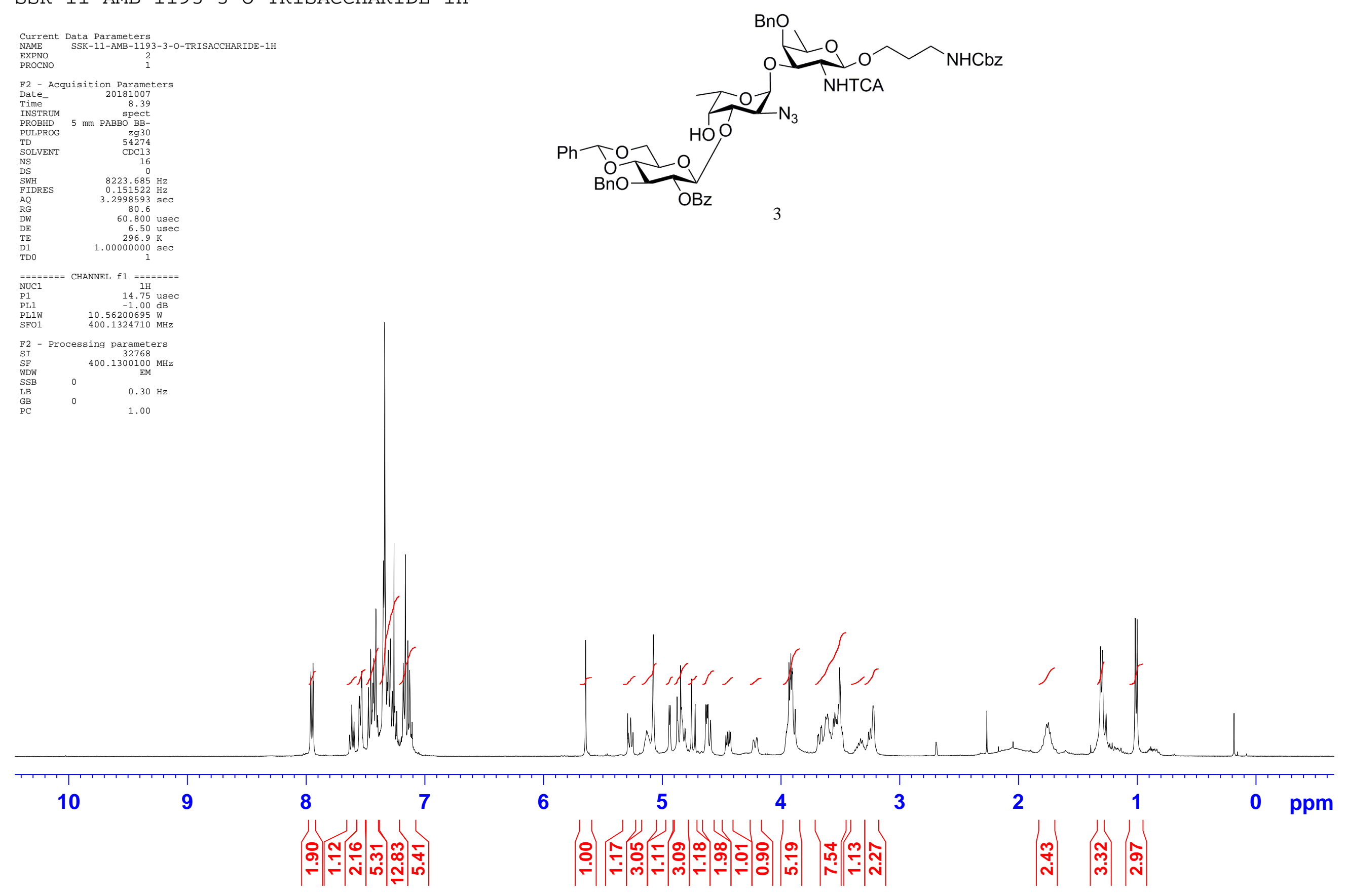
SSK-11-AMB-1193-3-0-TRISACCHARIDE-13C
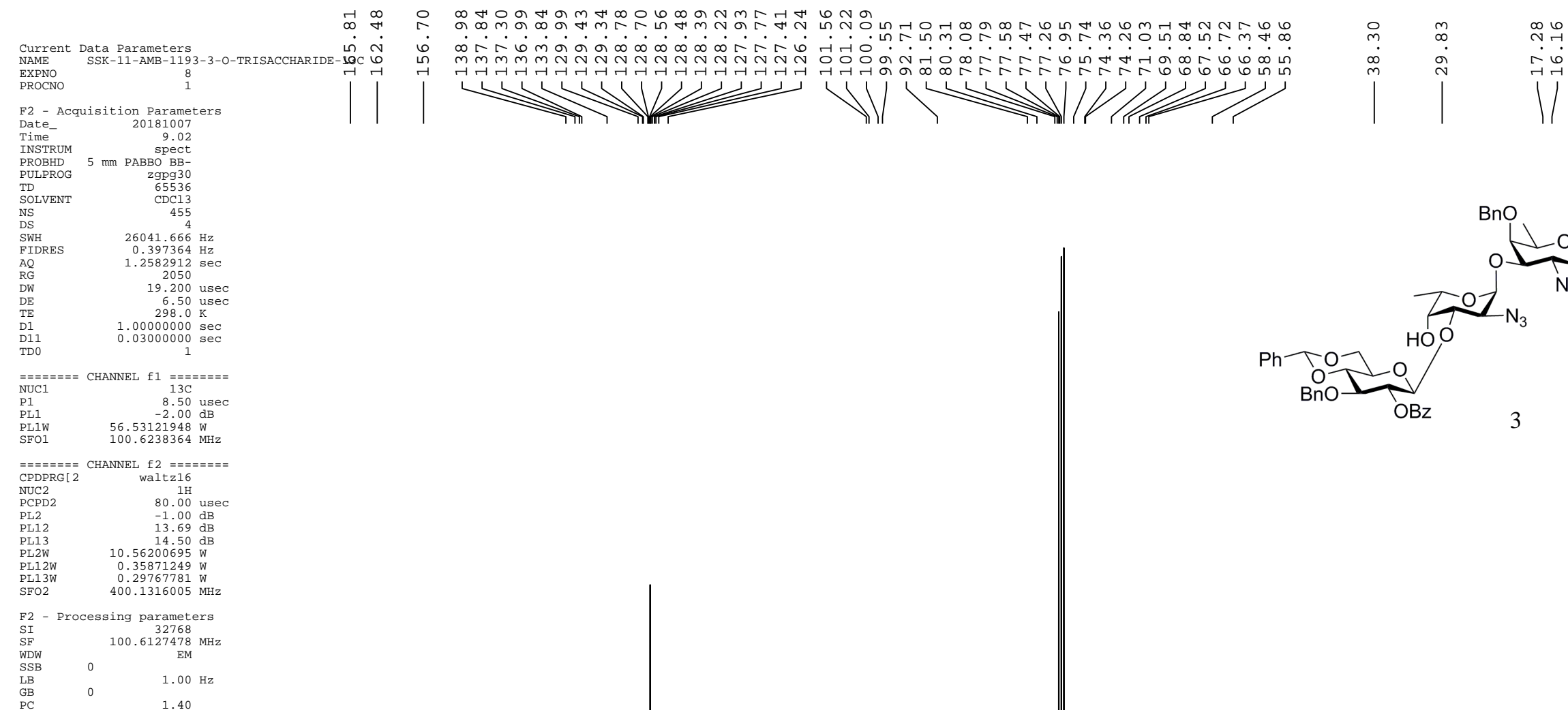

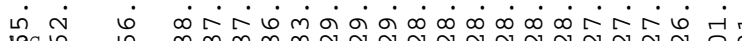

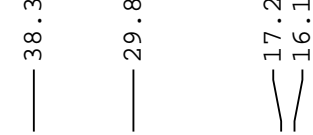

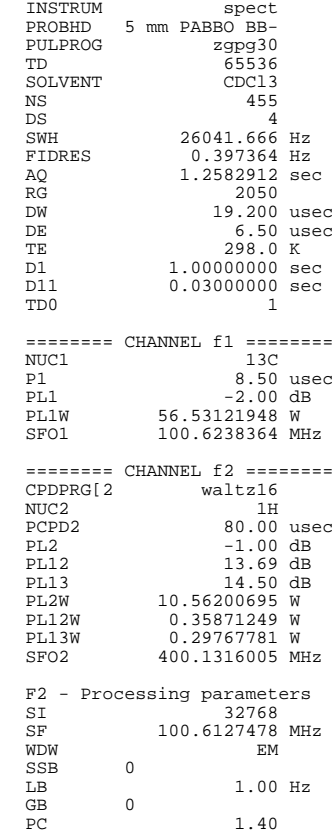

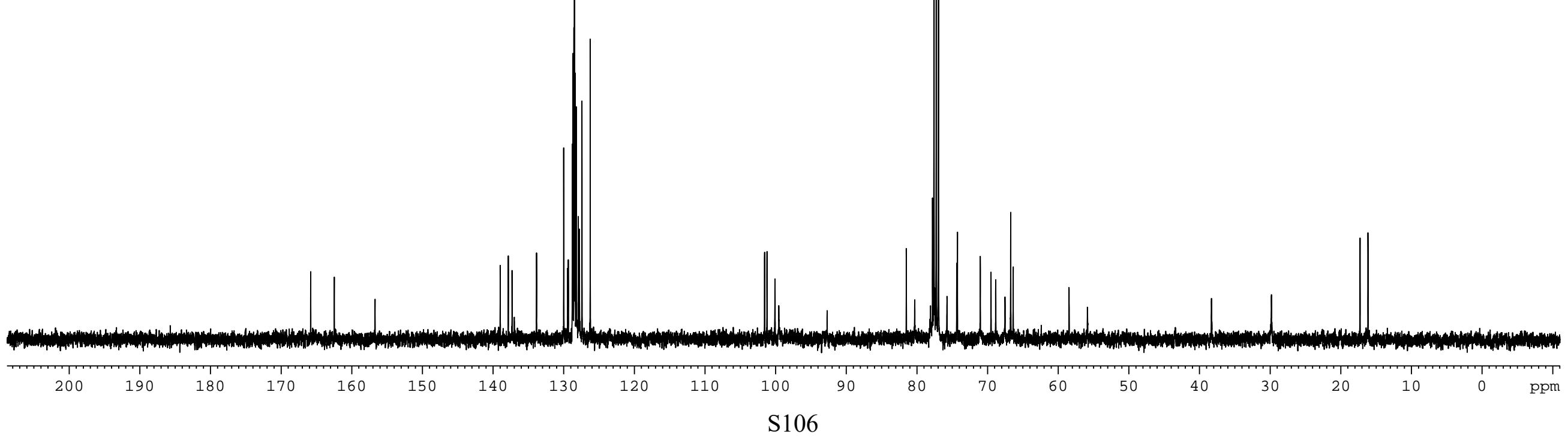


SSK - 11-AMB - 1193-3-0-TRISACCHARIDE - DEPT

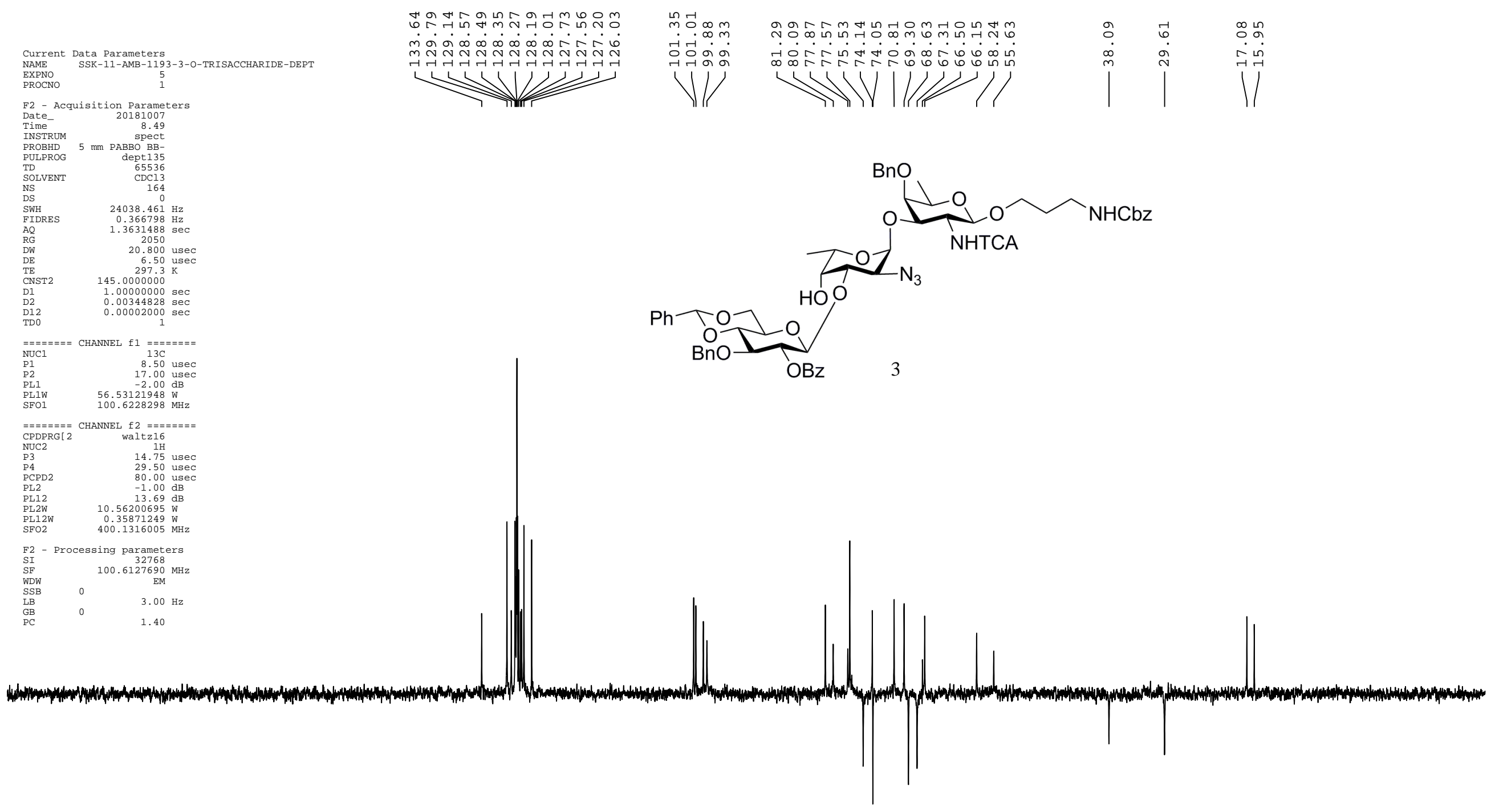

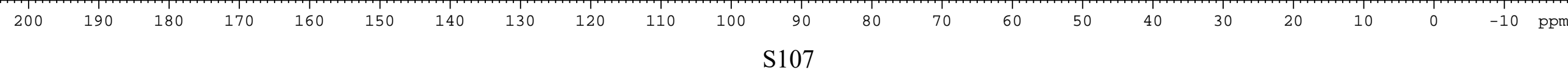


Current Data Parameters
NAME SSK-11-AMB-4-0-TRISACCHARIDE-1H

$\begin{array}{lr}\text { NAME } & \text { SSK-11-AMB-4-0- } \\ \text { EXPNO } & 1 \\ \text { PROCNO } & 1\end{array}$

F2 - Acquisition Parameters

Date
Time
Timstom

INSTRUM 21.33

PROBHD $5 \mathrm{~mm}$ PABBO BB/
PULPROG

PULPROG

SOLVENT

DS
SWH
FIDRES

$\begin{array}{lr}\text { AQ } & 3.152588 \mathrm{HZ} \\ \text { RG } & 98.91\end{array}$

DW $\quad 50.000$ usec

$\begin{array}{lr}\text { TE } & 297.6 \mathrm{~K} \\ \text { D1 } & 1.00000000 \mathrm{sec}\end{array}$

$========$ CHANNEL $\mathrm{f} 1 \quad========$

$\begin{array}{lr}\text { SF01 } & 500.1330885 \mathrm{MHZ} \\ \text { NUC1 } & 1 \mathrm{H}\end{array}$

$\begin{array}{lr}\text { NUC1 } & 13.35 \text { usec } \\ \text { PLW1 } & 16.00000000 \mathrm{~W}\end{array}$

F2 - Processing parameters

$\begin{array}{ll}\text { SI } & 65536 \\ \text { SF } & 500.1300132 \mathrm{MHZ}\end{array}$

$\begin{array}{lll}\text { WDW } & & \\ \text { SSB } & 0 & 0.30 \mathrm{~Hz} \\ \text { LB } & 0 & \end{array}$

$\begin{array}{lll}\mathrm{GB} & 0 & 1.00\end{array}$
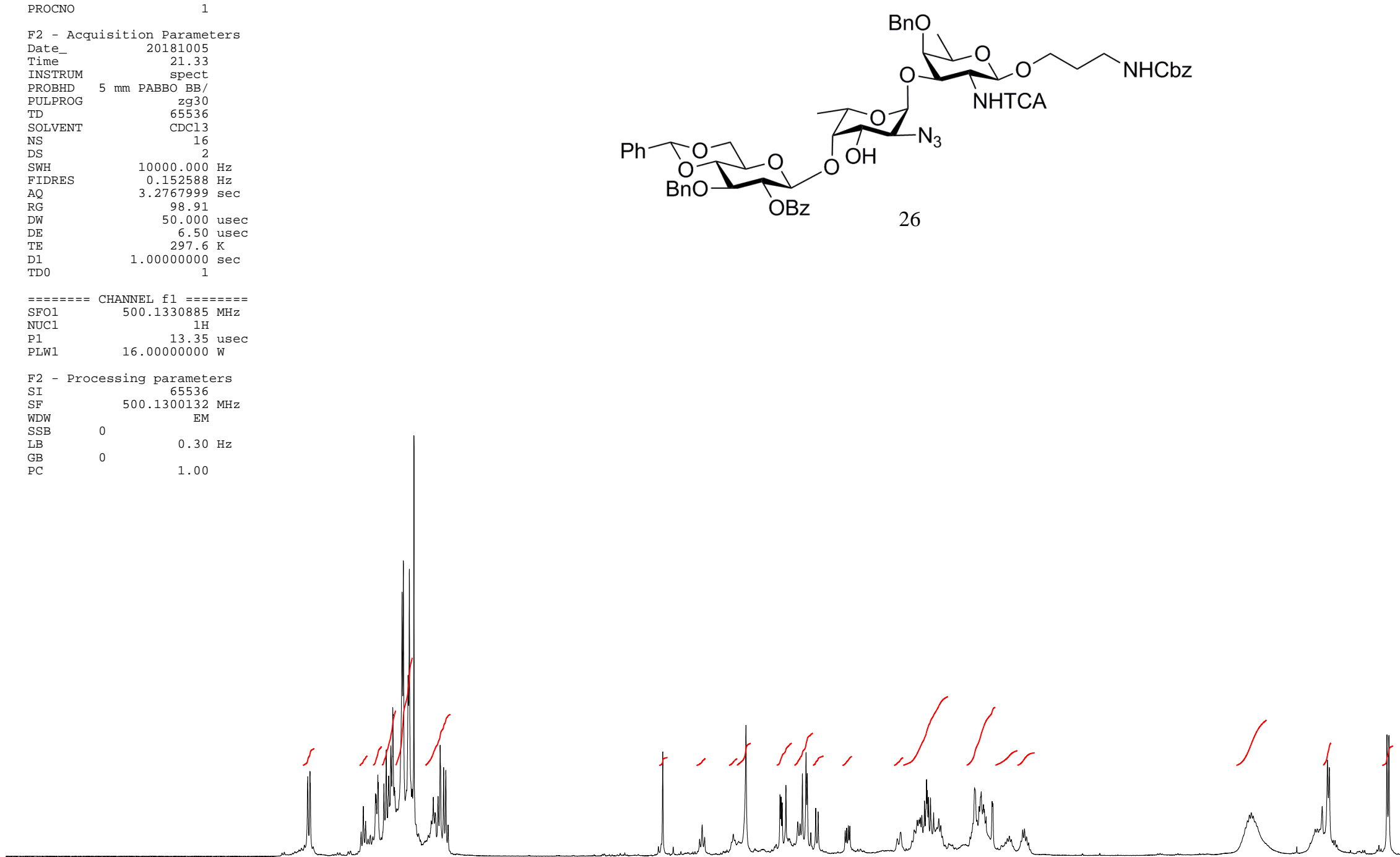

9

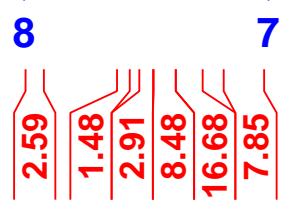

6

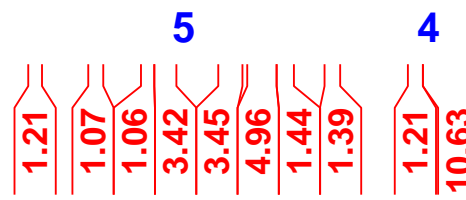

3 
SSK - 11 - AMB - 4 - 0 - TRISACCHARIDE - 13C

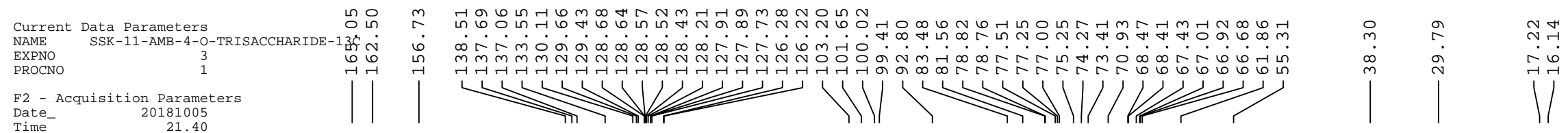

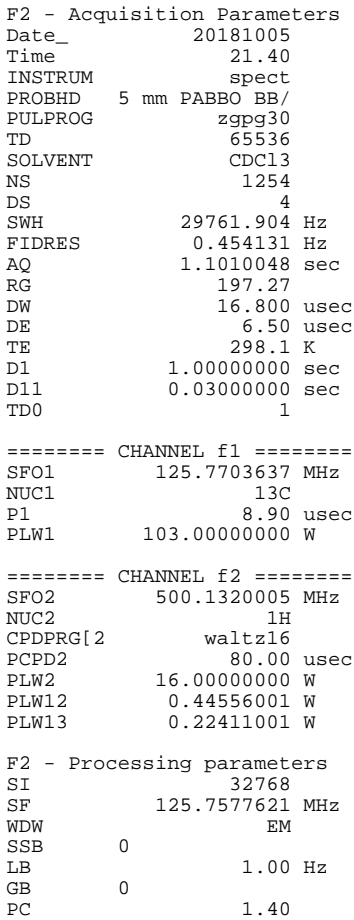

(N)
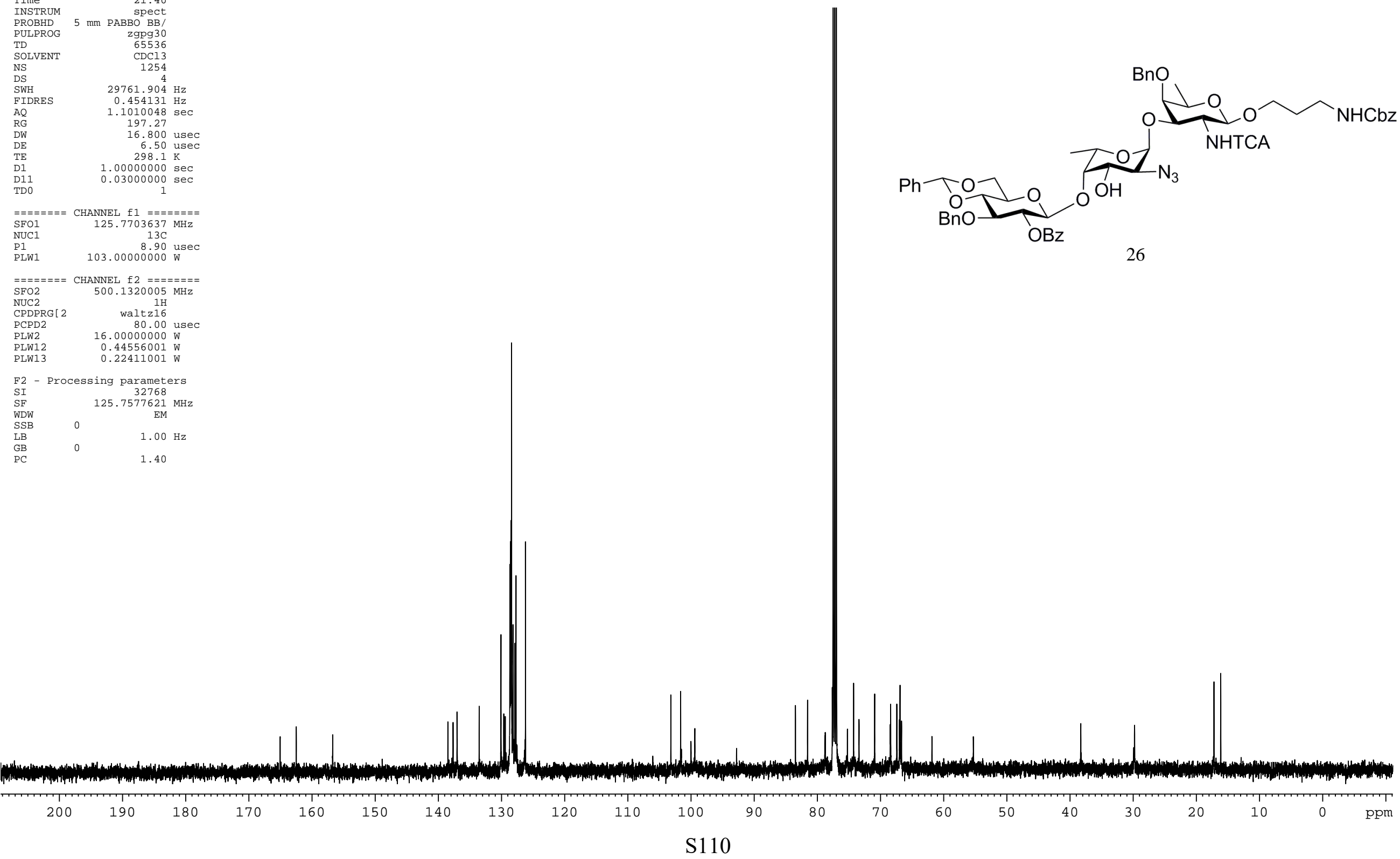
SSK - 11 - AMB - 1193 - 4 - 0 - TRISACCHARIDE - DEPT

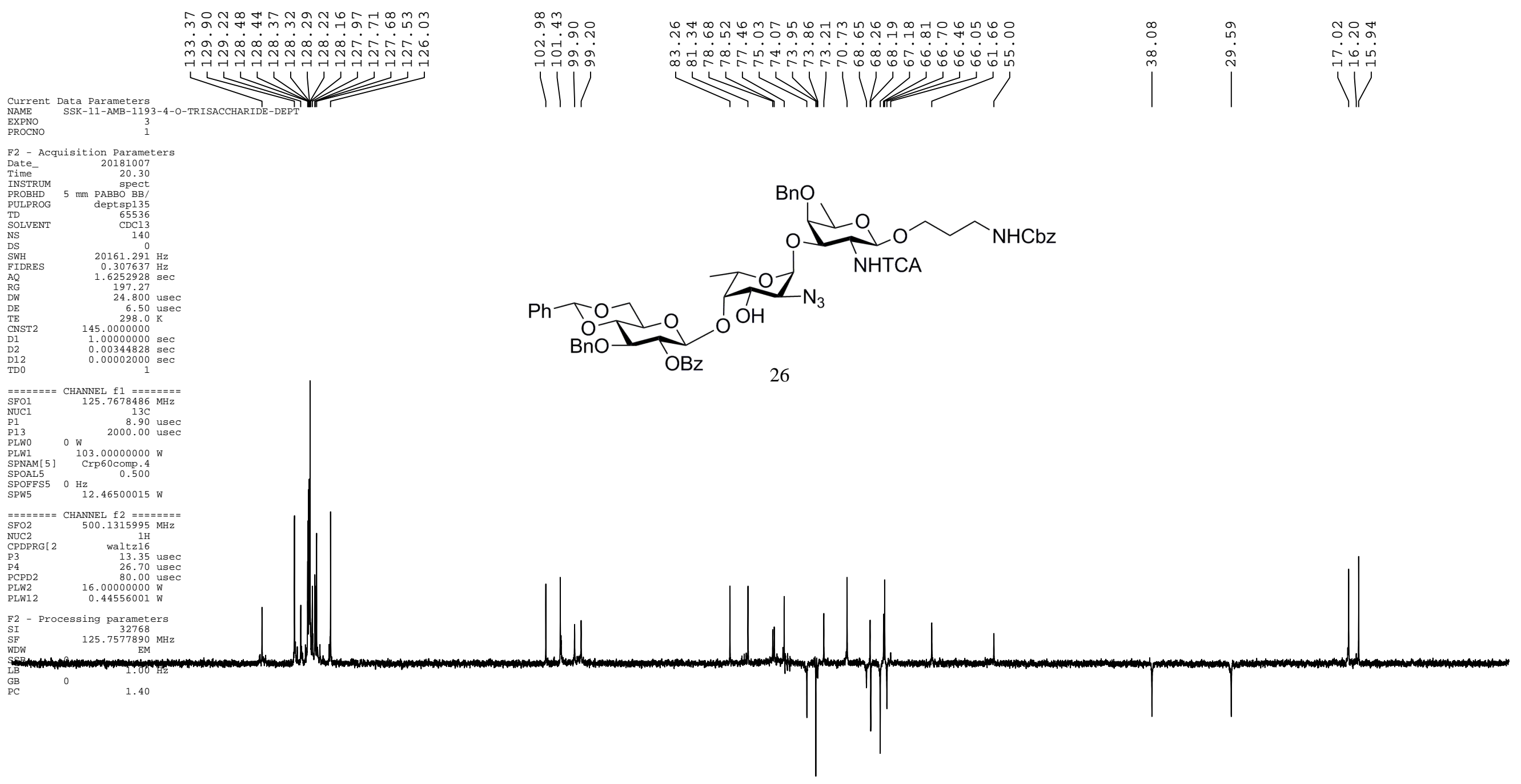

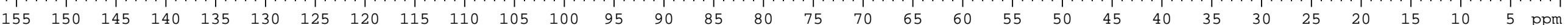




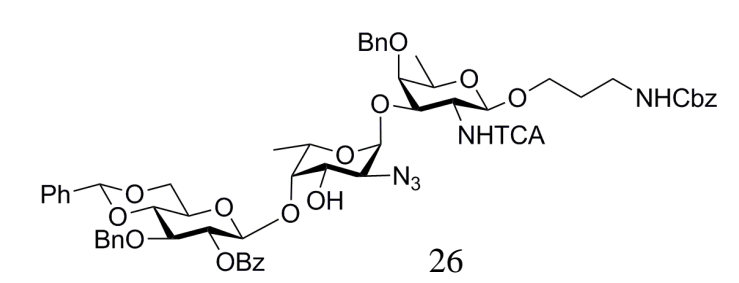

C of benzilidine

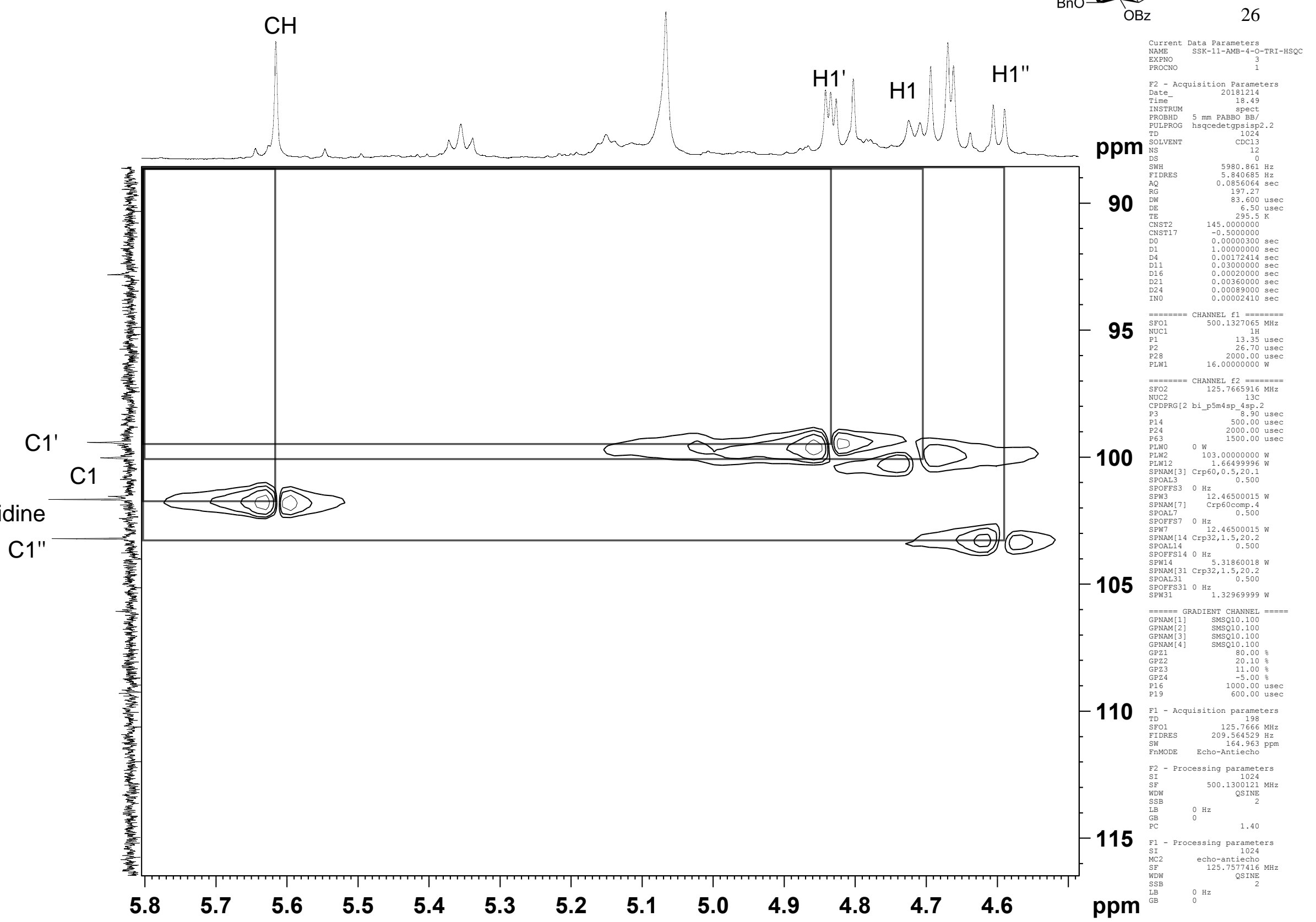


Current Data Parameters

NAME SSK-11-AMB-1215-1-O-ACETYLATED-TRI-1H

PROCNO

F2 - Acquisition Parameters

Date_ 20181112

Time 20.20

INSTRUM

PROBHD $5 \mathrm{~mm}$ PABBO BB-

$\begin{array}{ll}\text { PULPROG } & \text { zg30 } \\ \text { TD } & 54274\end{array}$

TD

TOLVENT

NS 22

DS $\quad 0$.

$8223.685 \mathrm{~Hz}$

FIDRES $0.151522 \mathrm{~Hz}$

AQ $\quad 3.2998593 \mathrm{sec}$

RG 161

$\begin{array}{ll}\text { DW } & 60.800 \text { usec } \\ \text { DE } & 6.50 \text { usec }\end{array}$

$\begin{array}{lc}\text { TE } & 296.4 \mathrm{~K} \\ \text { D1 } & 1.00000000 \\ \text { sec }\end{array}$

TD0 1

$========$ CHANNEL $\mathrm{f} 1$ ========

$\begin{array}{lc}\text { NUC1 } & \text { 1H } \\ \text { P1 } & 14.75 \text { usec }\end{array}$

PL1 $\quad-1.00 \mathrm{~dB}$

PL1W 10.56200695 W

SFO1 400.1324710 MHz

F2 - Processing parameters

$\begin{array}{lc}\text { SI } & 32768 \\ \text { SF } & 400.1300102 \\ \text { MHz }\end{array}$

WDW EM

SSB 0

LB $\quad 0.30 \mathrm{~Hz}$

$\begin{array}{lll}\text { GB } & 0 & \\ \text { PC } & & 1.00\end{array}$

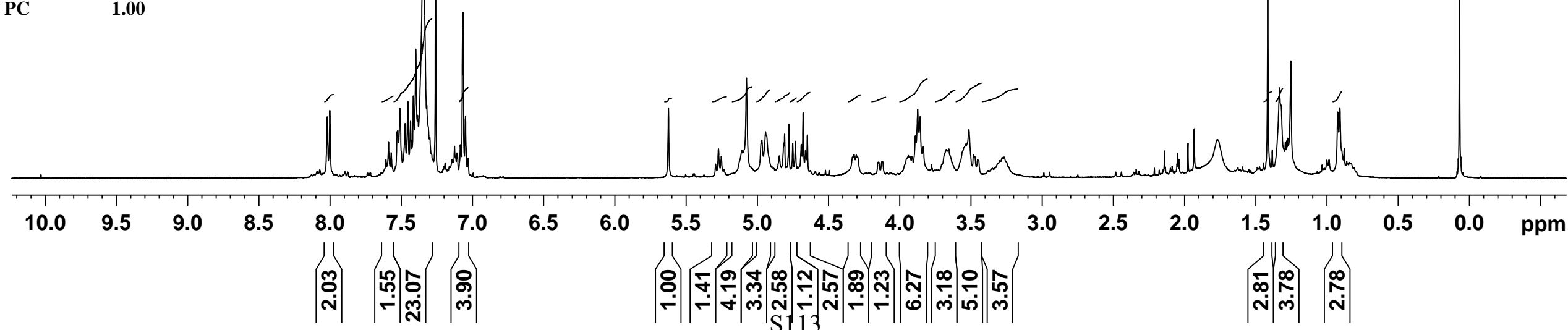


SSK-11 - AMB - 1215 - 13C

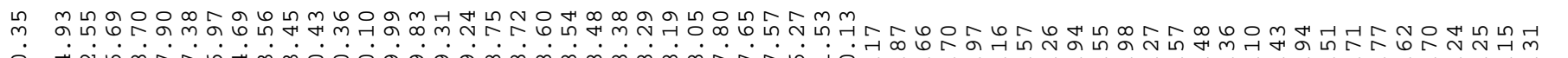

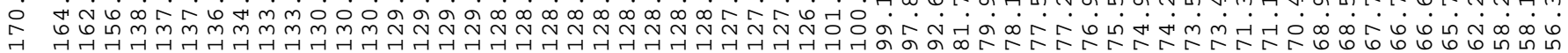

Current Data Parameters
NAME SSK-11-AMB-1215-13C

EXPNO
PROCNO

F2 - Acquisition Parameters

\begin{tabular}{lr} 
Date & Acquisition Parame \\
Time & 20181115 \\
\hline
\end{tabular}

Time
INSTRUM

4.44
spect

PROBHD $5 \mathrm{~mm}$ PABBO BB
PULPROG

TD

65536
CDC13

NS

$\begin{array}{ll}\text { DS } & \\ \text { SWH } & 24038.461 \\ \end{array}$

$\begin{array}{ll}\text { FIDRES } & 24038.461 \mathrm{~Hz} \\ \text { AO } & 0.366798 \mathrm{~Hz}\end{array}$

AQ $\quad 1.3631488 \mathrm{sec}$

$\begin{array}{lr}\mathrm{DW} & 20.800 \mathrm{usec} \\ \mathrm{DE} & 6.50 \mathrm{usec}\end{array}$

TE $\quad 296.6 \mathrm{~K}$

$\begin{array}{ll}\text { D1 } & 1.00000000 \mathrm{sec} \\ \text { D11 } & 0.03000000 \mathrm{sec}\end{array}$

TDQ

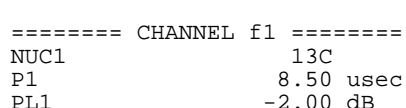

$\begin{array}{lr}\text { P1 } & 8.50 \text { usec } \\ \text { PL1 } & -2.00 \mathrm{~dB} \\ \text { PL1W } & 56.53121948 \mathrm{~W} \\ \text { SF01 } & 100.6238364 \mathrm{MHz}\end{array}$

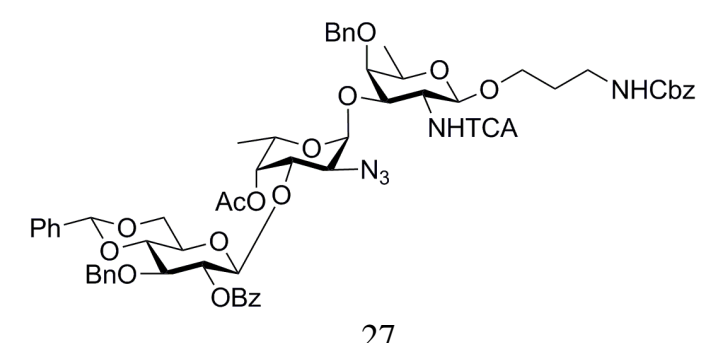

$=======$ CHANNEL $\mathrm{f} 2$ =======+

CPDPRG [2 waltz16

NUC2 2

PL2

PL13

PL2W
PL12W
PL13W

$\mathrm{PL} 13 \mathrm{~W}$
$\mathrm{SFO2}$

80.00 usec
$-1.00 \mathrm{~dB}$

$13.69 \mathrm{~dB}$

$10.56200695 \mathrm{~W}$

$10.56200695 W$
0.35871249

$0.29767781 \mathrm{~W}$
$400.1316005 \mathrm{MHz}$

F2 - Processing parameters

$\begin{array}{lr}\text { SI } & 32768 \\ \text { SF } & 100.6127461 \mathrm{MHz}\end{array}$

WDW $\quad 0 \quad$ EM

$\begin{array}{lll}\text { SSB } & 0 & 1.00 \mathrm{~Hz} \\ \mathrm{LB} & 0 & \end{array}$

$\mathrm{PC} \quad 1.40$

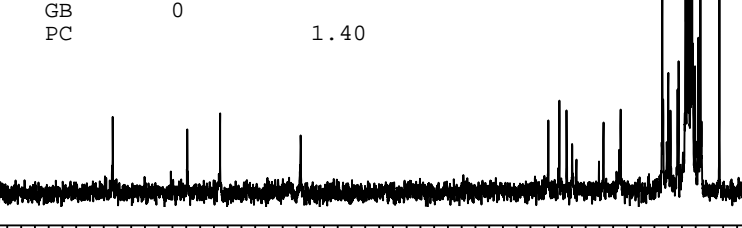

$170 \quad 160 \quad 150$

$50 \quad 140$

130

120

110

100

90

80

70

60

50

40

30

20

10 
SSK - 11 - AMB - 1215 - 1 - 0 - ACETYLATED - TRI - HHCOSY

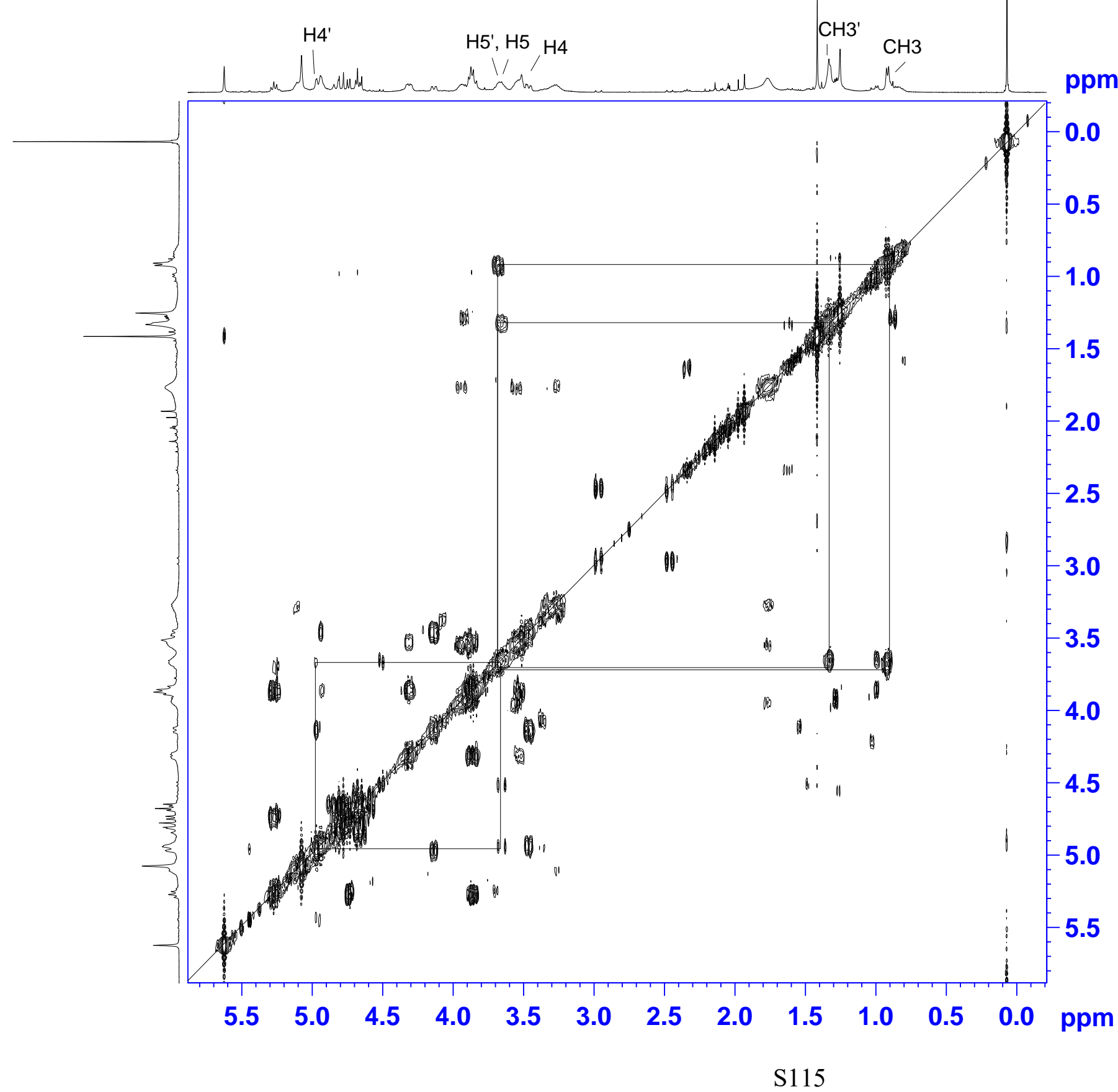

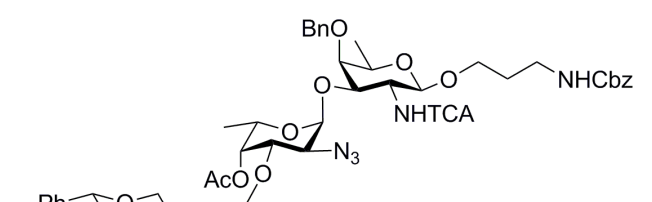

Ph $\mathrm{AcO}$

27

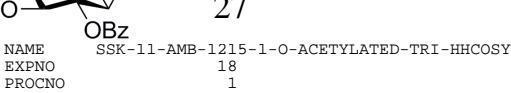

F2-Acquisition Parameters

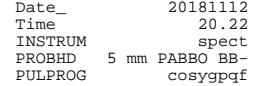

SOLVENT
NS

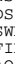

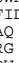

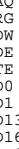

0.002000
0.00000
sec
0.00041000
sec

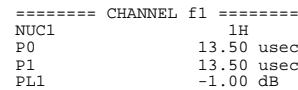

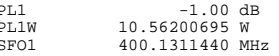

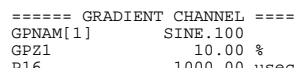

TD - Acquisition parameters
SF01

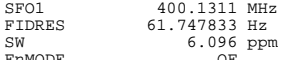

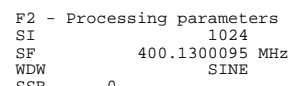

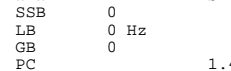

$\begin{array}{lc}\mathrm{CB} & 1.40 \\ \mathrm{PC} & \\ \mathrm{FI} & - \text { Processing parameters } \\ \mathrm{SI} & 1024\end{array}$

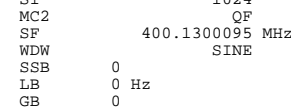




\section{SSK-11-AMB-1209-1H}

Current Data Parameters

NAME SSK-11-AMB-1209-1H

EXPNO

PROCNO

F2 - Acquisition Parameters

Date_ 20181028

INSTRUM

PROBHD $5 \mathrm{~mm}$ PABBO BB

PULPROG zg30

TD 65536

SOLVENT ${ }^{65536} \mathrm{CDCl}$

NS 10

SWH $\quad 10000.000 \mathrm{~Hz}$

FIDRES $\quad 0.152588 \mathrm{~Hz}$

AQ $\quad 3.2767999 \mathrm{sec}$

RG $\quad 61.42$

DW 50.000 usec

DE $\quad 6.50$ use

TE $\quad 295.5 \mathrm{~K}$

D1 $\quad 1.00000000 \mathrm{sec}$

TDO 1

$========$ CHANNEL $\mathrm{f1}========$

SFO1 500.1330885 MHz

NUC1 $1 \mathrm{H}$

$\begin{array}{ll}\text { P1 } & 13.35 \text { usec } \\ \text { PLW1 } & 16.00000000\end{array}$

F2 - Processing parameters

$\begin{array}{lc}\text { SI } & 65536 \\ \text { SF } & 500.1300139 \\ \text { MHz }\end{array}$

WDW EM

$\begin{array}{lll}\text { LSB } & 0 & \\ \text { CB } & 0 & 0.30\end{array}$

$\begin{array}{lll}\text { GB } & 0 & 0.30\end{array}$

PC

1.00
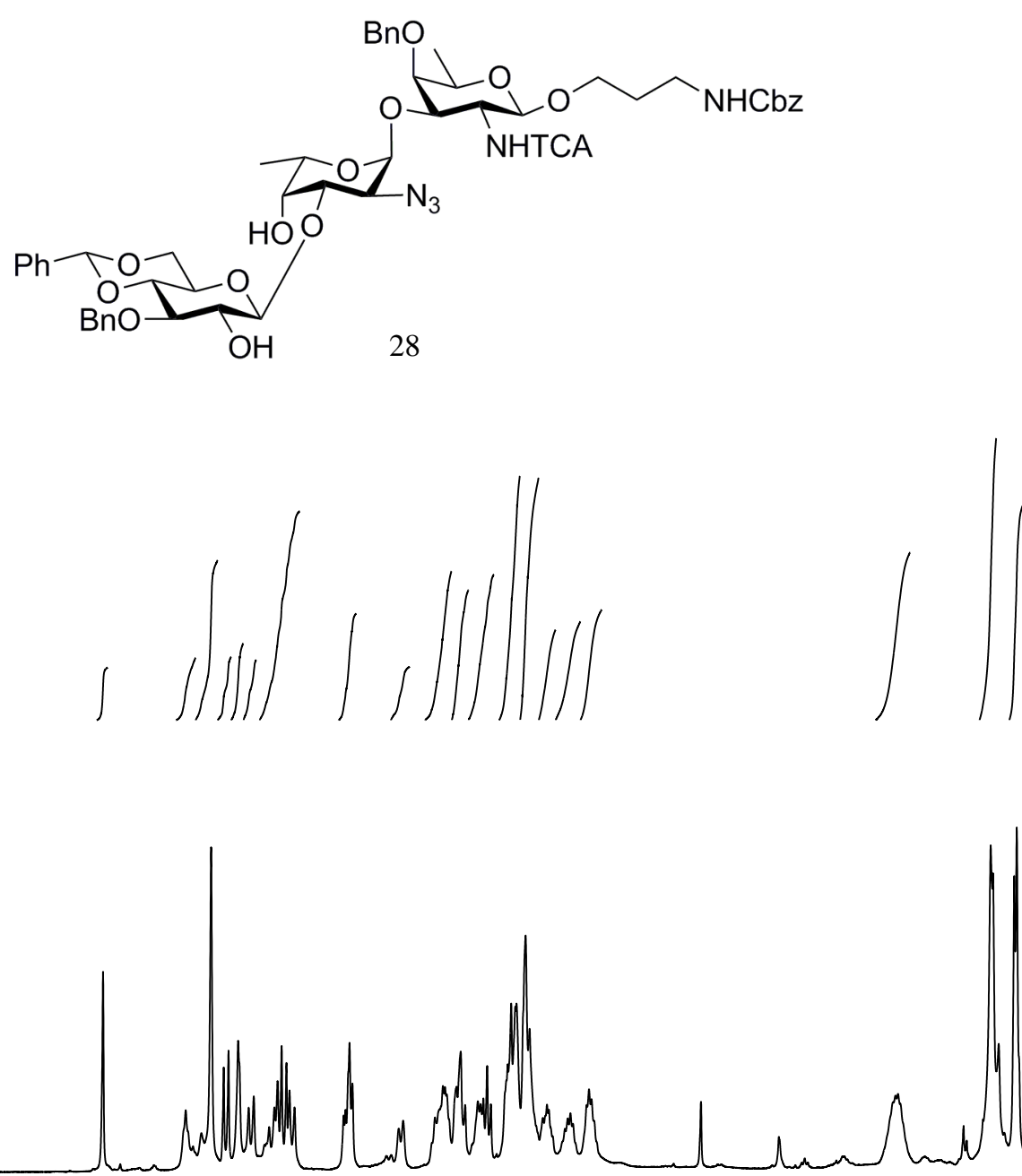

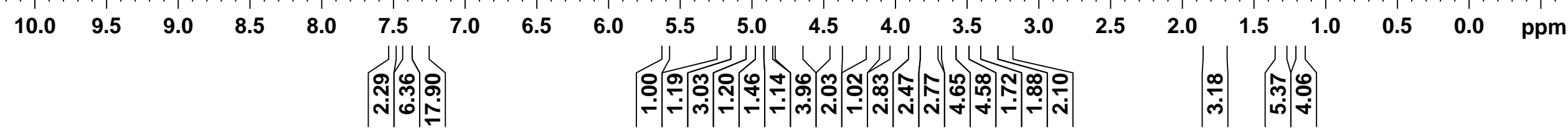


SSK-11-AMB - 1209-13C

Current Data Parameters NAME SSK-11-AMB-1209-13C EXPNO

PROCNO

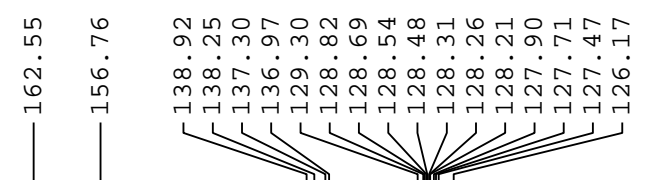

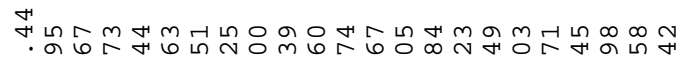

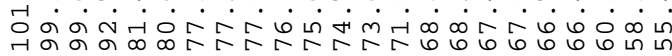

F2 - Acquisition Parameters

Date

20181029

INSTRUM

PROBHD

PULPROG

$5 \mathrm{~mm}$ PABBect

PULPROG Zgpg30

TD $\quad 65536$

CDCl3

NS 335

SWH $29761.904 \mathrm{~Hz}$

$0.454131 \mathrm{~Hz}$

AQ $\quad 1.1010048 \mathrm{sec}$

DW

DE

16.800 usec

6.50 usec

D1 $1.00000000 \mathrm{sec}$

D11 $\quad 0.03000000 \mathrm{sec}$

$======$ CHANNEL $\mathrm{f} 1$ =======

SF01 $125.7703637 \mathrm{MHz}$

$\begin{array}{lr}\text { NUC1 } 13 \mathrm{C} \\ \mathrm{P} 1 & 8.90 \text { usec }\end{array}$

PLW1 $103.00000000 \mathrm{~W}$

$=======$
SF02
SHANNEL $\mathrm{f} 2========$

SF02

NUC2

CPDPRG $[2$
PCPD2

PLW2

$$
\text { waltz16 }
$$

80.00 usec

16.0000000० W

PLW13

$0.44556001 \mathrm{~W}$

$\odot .22411001 \mathrm{~W}$

F2 - Processing parameters

SI 32768

$\begin{array}{lr}\text { SF } & 125.7577648 \mathrm{MHz}\end{array}$

SSB

$\odot$

$1.00 \mathrm{~Hz}$

GB

PC

$\odot$

1.40

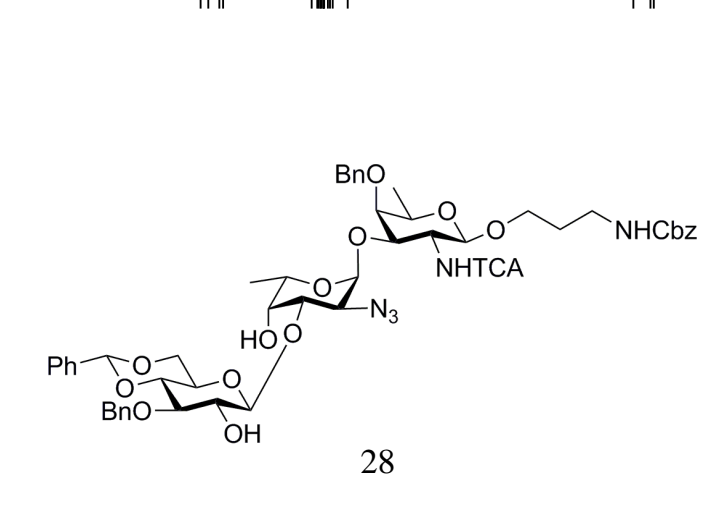

$\stackrel{4}{5} \quad \hat{N} \frac{4}{2}$

$1120 \times 111$

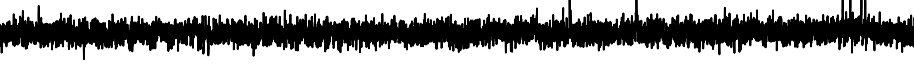


Current Data Parameters
NAME SSK-11-AMB-1209-DEPT
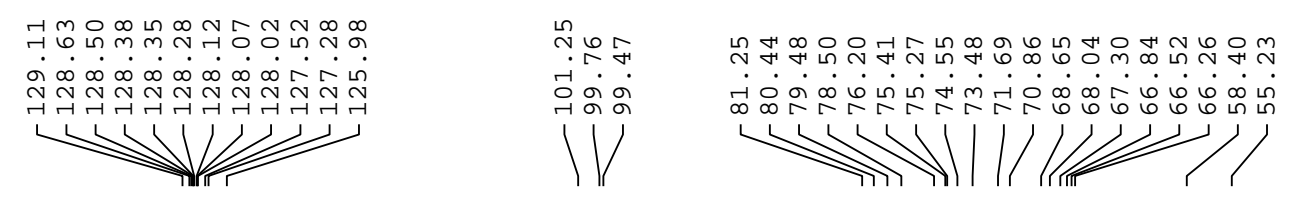

\section{i}

$$
\text { EXPNO }
$$$$
\begin{aligned}
& 3 \\
& 1
\end{aligned}
$$

F2 - Acquisition Parameters

$\begin{array}{lr}\text { Date_ } & 20181029 \\ \text { Time } & 0.20\end{array}$

INSTRUM spect

PULPROG deptsp135

TD 65536

$\begin{array}{lr}\text { SOLVENT } & \text { CDC13 } \\ \text { NS } & 115\end{array}$

DS

SWH
FIDRES

FIDRES
AQ
RG
DW

DW

TE
CNST2

D1

D2
D12

$\mathrm{D} 12$
TDO

$=======$ CHANNEL $\mathrm{f} 1 \mathrm{=}=====\overline{=}$

SF01

P13

PLWO

PLW1

$20161.291 \mathrm{~Hz}$

$0.307637 \mathrm{~Hz}$

800 usec 6.50 usec
$296.4 \mathrm{~K}$

145.0000000

1. $00000000 \mathrm{sec}$

0.00344828 sec

CHANNELL $f 1$.

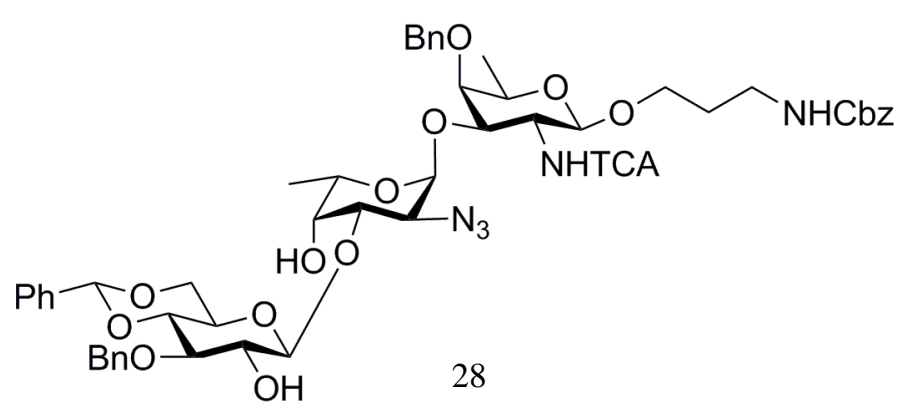

103.000000000
Crp60comp.4

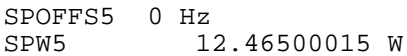

$\begin{array}{lr}======= & \text { CHANNEL } \mathrm{f} 2======= \\ \text { SF02 } & 500.1315995 \mathrm{MHz} \\ \text { NUC2 } & 1 \mathrm{H} \\ \text { CPDPRG }[2 & \text { waltz16 } \\ \text { P3 } & 13.35 \mathrm{usec} \\ \text { P4 } & 26.70 \mathrm{usec} \\ \text { PCPD2 } & 80.00 \mathrm{usec} \\ \text { PLW2 } & 16.00000000 \mathrm{~W} \\ \text { PLW12 } & 0.44556001 \mathrm{~W}\end{array}$

$44556001 \mathrm{~W}$

F2 - Processing parameters

$\begin{array}{lr}\text { SI } & 32768 \\ \text { SF } & 125.7577890 \mathrm{MHz}\end{array}$

WDW

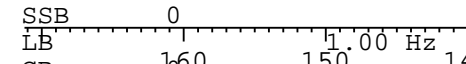

GB $\quad 50 \quad 150$

$140 \quad 130$

110

100

$9 \odot$

80

70

60

50

40

30

20

$10 \quad 0 \quad \mathrm{ppm}$ 
Current Data Parameters

NAME SSK-11-AMB-1212-1H

EXPNO

1

F2 - Acquisition Parameters

$\begin{array}{lr}\text { Date_ } & 20181102 \\ \text { Time } & 2.17\end{array}$

2.17

spect
INSTRUM $5 \mathrm{~mm} \mathrm{PABBO}$ BB-

PULPROG 5 ma

$\begin{array}{lr}\text { TD } & \text { Zg30 } \\ & 54274\end{array}$

SOLVENT CDCl3

13
0

SWH $\quad 8223.685 \mathrm{~Hz}$

FIDRES $\quad 0.151522 \mathrm{~Hz}$

AQ 3.2998593 sec

RG 161

DW $\quad 60.800$ usec

$\quad 6.50$ usec

$296.5 \mathrm{~K}$

$1.00000000 \mathrm{sec}$

$=======$ CHANNEL f1 =====ニ=

NUC1

PL1 $1 \mathrm{H}$

SF01 $400.1324710 \mathrm{MHz}$

F2 - Processing parameters

SI 32768

SF $\quad 400.1300102 \mathrm{MHz}$

WDW

SSB

LB

$\mathrm{GB}$

EM

$0.30 \mathrm{~Hz}$

0
0

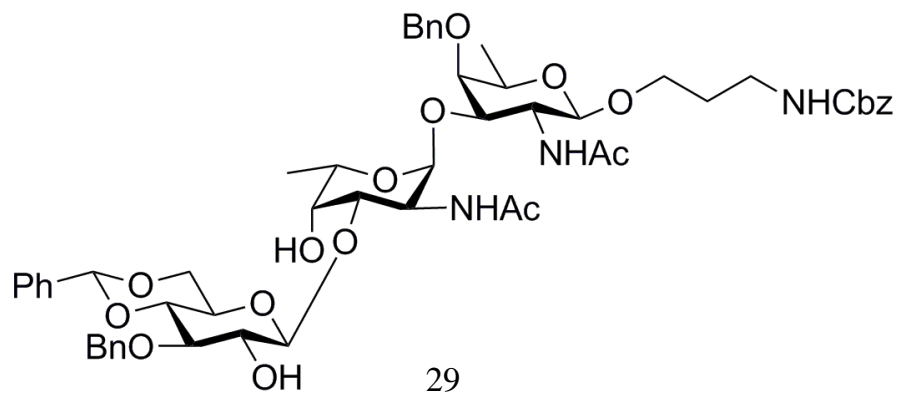

9

8

6

$\left|\begin{array}{l}0 \\ 4 \\ \hdashline\end{array}\right|$

5

4

3

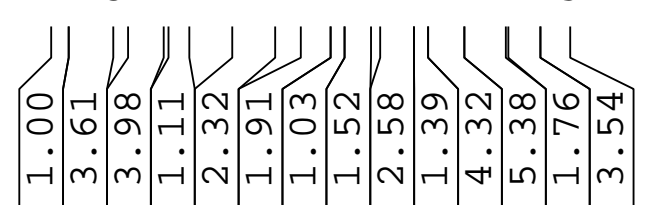

S119

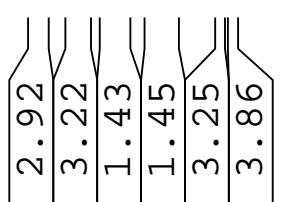

1

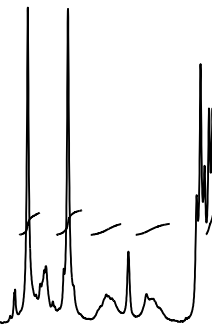

2

$\odot$

ppm

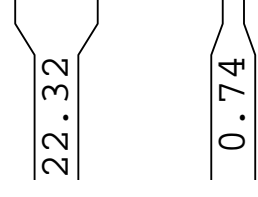


Current Data Parameters
NAME

NAME
EXPNO

1

F2 - Acquisition Parameters

$\begin{array}{lr}\text { Date } & \text { Acquisition } \\ \text { Time } & 20181102 \\ & 9.44\end{array}$

INSTRUM $5 \mathrm{mmect}$

PROBHD
PULPROG

$$
\begin{array}{r}
\text { zgpg30 } \\
65536 \\
\operatorname{CDCl} 13
\end{array}
$$

SOLVENT

DS

SWH $\quad 070$

FIDRES $\quad 0.454131 \mathrm{~Hz}$

$\begin{array}{ll}\text { AQ } & 1.1010048 \mathrm{sec} \\ \text { RG } & 197.27\end{array}$

$\begin{array}{lr}\text { DW } & 16.800 \mathrm{usec} \\ \mathrm{DE} & 6.50 \mathrm{usec}\end{array}$

TE $\quad 297.7 \mathrm{~K}$

$\begin{array}{ll}\text { D1 } & 1.00000000 \mathrm{sec} \\ \text { D11 } & 0.03000000 \mathrm{sec}\end{array}$

$=======$ CHANNEL $f 1$ = $===== \pm=$ SF01 $\quad 125.7703637 \mathrm{MHZ}$

P1 8.90 usec

$\begin{array}{ll}\text { P1 } & 8.90 \text { usec } \\ \text { PLW1 } & 103.00000000 \mathrm{~W}\end{array}$

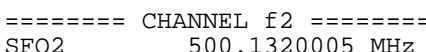

$\begin{array}{lr}\text { NUC2 } & 500.132005 \\ \text { CPDPRG [2 } & \text { waltz16 }\end{array}$

$\begin{array}{lc}\text { CPDPRG 2 } & \text { waltz16 } \\ \text { PCPD2 } & 80.00 \text { usec }\end{array}$

PLW2 16. $00000000 \mathrm{~W}$

PLW13 $\quad 0.22411001 \mathrm{~W}$

F2 - Processing parameters

$\begin{array}{lr}\text { SI } & 32768 \\ \mathrm{SF} & 125.7577639 \mathrm{MHz}\end{array}$

WDW $\quad 0 \quad E M$

$\begin{array}{ll}\mathrm{LB} & 1.00 \mathrm{~Hz}\end{array}$

$\begin{array}{lll}\mathrm{GB} & \mathrm{P} & \\ \mathrm{PC} & & \end{array}$

1.40

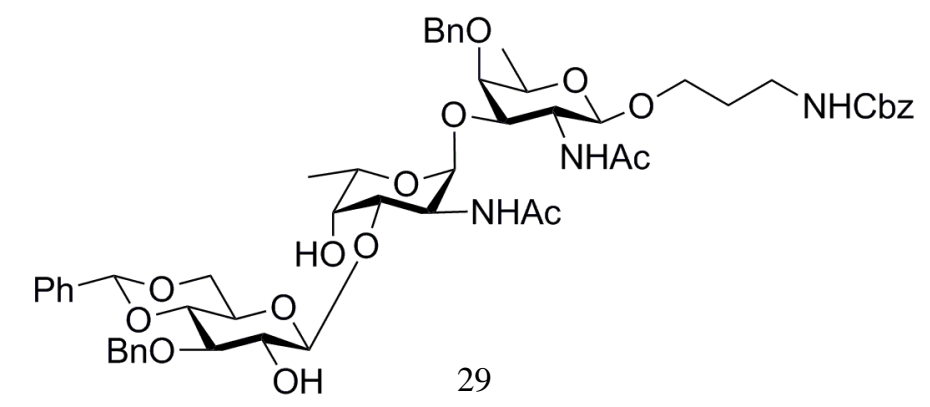

\begin{tabular}{|c|c|c|c|c|c|c|c|c|c|c|c|c|c|c|c|c|c|c|c|c|}
\hline 200 & 190 & 180 & 170 & 160 & 150 & 140 & 130 & 120 & 110 & 100 & 90 & 80 & 70 & 60 & 50 & 40 & 30 & 20 & 10 & 0 \\
\hline
\end{tabular}


SSK - 11 - AMB - 1212 - DEPT

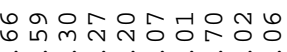
ज્ञ

Current Data Parameters
NAME SSK-11-AMB-1212-DEPT
EXPNO

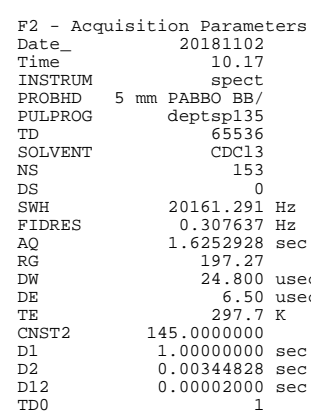

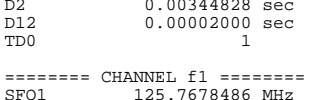

$\begin{array}{lr}\text { SF01 } & 125.7678486 \mathrm{MHz} \\ \text { NUC1 } & 13 \mathrm{C} \\ \text { P1 } & 8.90 \text { usec } \\ \text { P13 } & 2000.00 \text { usec }\end{array}$

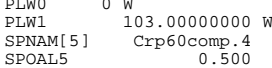

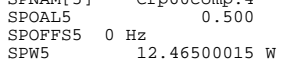

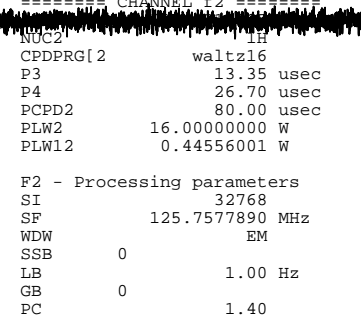

ํํㅇํํํํㅁำ

家

11

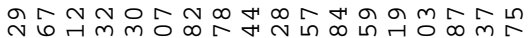

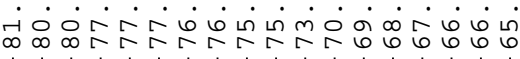
$\longrightarrow$

$\stackrel{0}{\bullet}$

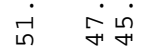

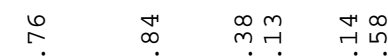

i $\quad \dot{N} \quad \dot{N}$ ते

${ }^{\infty}$

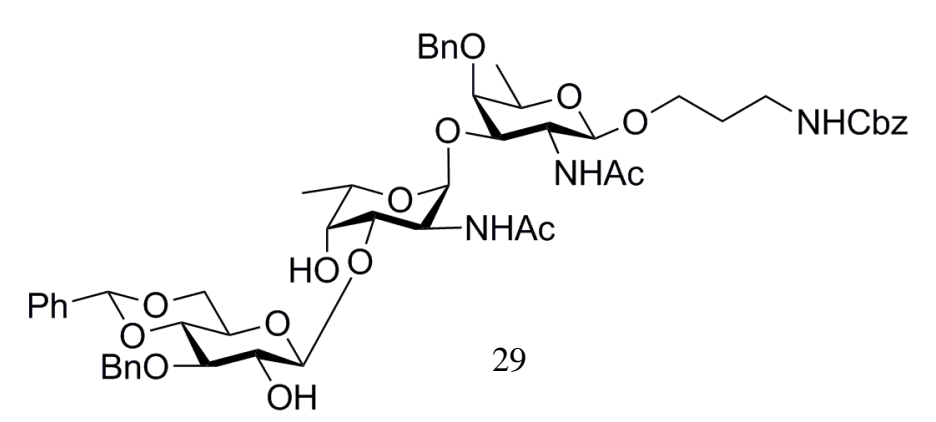


Current Data Parameters

NAME SSK-11-AMB-1214-1H

EXPNO

3

F2 - Acquisition Parameters

Date_ 20181113

Time

0.35

INSTRUM spect

PABBO BB/

PULPROG Zg30

TD 65536

SOLVENT

$\begin{array}{lc}\text { NS } & 16 \\ \text { DS } & 0\end{array}$

DSH $\quad 10000.000 \mathrm{~Hz}$

$\begin{array}{lr}\text { FWH } & 10000.000 \mathrm{~Hz} \\ \text { FIDRES } & \mathbf{0 . 1 5 2 5 8 8} \mathrm{Hz}\end{array}$

$\begin{array}{lr}\text { FIDRES } & \mathbf{0 . 1 5 2 5 8 8} \mathrm{H} \\ \text { AQ } & 3.2767999 \mathrm{sec}\end{array}$

RG $\quad \mathbf{8 0 . 3 5}$

DW $\quad \mathbf{5 0 . 0 0 0}$ usec

DE $\quad 6.50$ usec

TE $\quad 297.1 \mathrm{~K}$

$\begin{array}{lc}\text { D1 } & 1.00000000 \mathrm{sec} \\ \text { TD0 } & 1\end{array}$

======== CHANNEL $\mathrm{f} 1$ ========

SFO1 500.1330885 MHz

NUC1 1H

P1 13.35 usec

F2 - Processing parameters

SI - Processing para

SF $\quad 500.1300000 \mathrm{MHz}$

WDW $\quad$ EM

WSB

$\begin{array}{lll}\text { SSB } & 0 & \\ \text { LB } & & 0.30 \mathrm{~Hz}\end{array}$

GB

$0.30 \mathrm{H}$

1.00

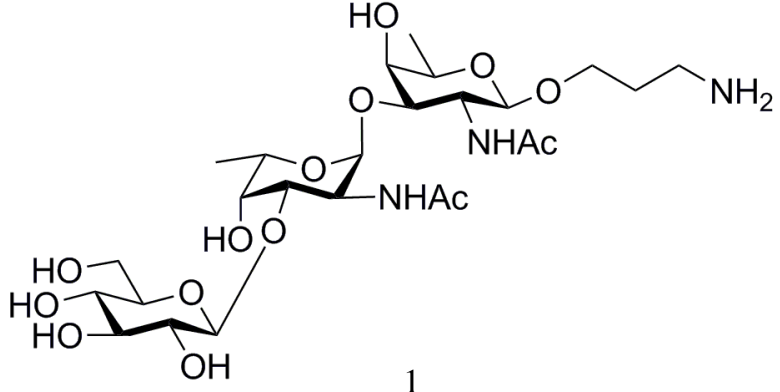

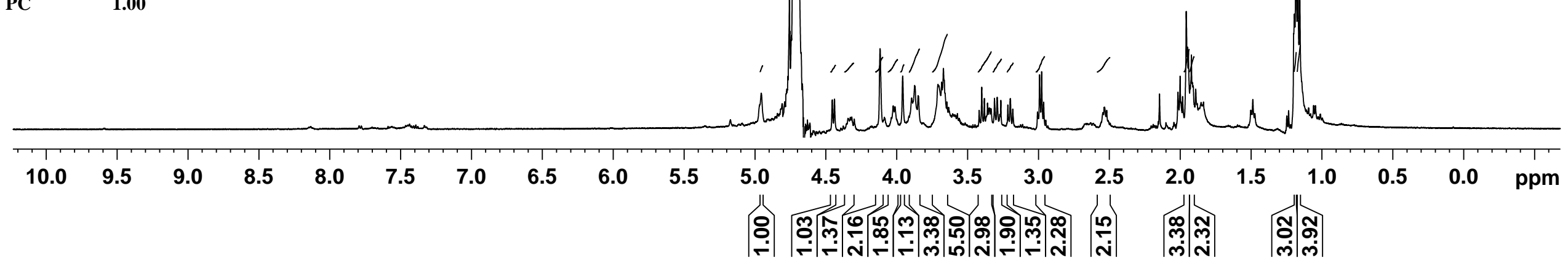


Current Data Parameters
NAME DSK-11-AMB-1214-13C

EXPNO

கे

PROCN

吾寺

F2 - Acquisititon Parameters

Date20.04110

PROBHD $5 \mathrm{~mm}$ PABCEO BB

PULPROG $29 \mathrm{mg} 30$

TD ${ }_{\text {NS }}{ }^{65536}$ D2O

$\begin{array}{ll}\text { NS } & 28647 \\ \text { DS } & 0\end{array}$

$\begin{array}{ll}\text { SWH } & 29761.904 \mathrm{~Hz} \\ \text { FIDRES } & 0.454131 \mathrm{~Hz}\end{array}$

AQ $\quad 1.1010048 \mathrm{sec}$

\begin{tabular}{ll}
$\mathrm{RG}$ & 197.27 \\
$\mathrm{DW}$ & 16.800 usec \\
\hline
\end{tabular}

$\begin{array}{ll} & \\ \text { DE } & 6.5050 \text { usec } \\ \text { TE } & 296.8 \mathrm{~K}\end{array}$

D1 $1.00000000 \mathrm{sec}$

TDo $0.03000000 \mathrm{sec}$

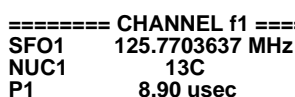

$\begin{array}{ll}\text { Nol } & 8.90 \text { usec } \\ \text { PLW1 } & 103.0000000 \mathrm{w}\end{array}$

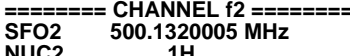

NUC2 $1 \mathrm{H} 1$

PCPD2 80.00 usec

PLW12 $16.00000000 \mathrm{~W}$

$\begin{array}{ll}\text { PLW12 } & 0.44556001 \mathrm{~W} \\ \text { PLW13 } & 0.22411001 \mathrm{~W}\end{array}$

F2- Processing parameters

$\begin{array}{ll}\text { SI } & 32768 \\ \text { SF } & 125.7577890 \mathrm{MHz}\end{array}$

WSB 0 EM

$\begin{array}{lll}\mathrm{SSB} & 0 & 2.00 \mathrm{~Hz} \\ \mathrm{LB} & & \end{array}$

$\begin{array}{lll}\mathrm{GB} & 0 & 1.40\end{array}$

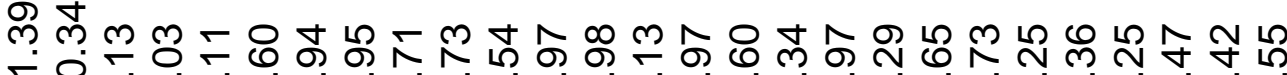

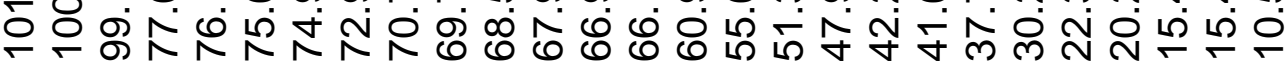
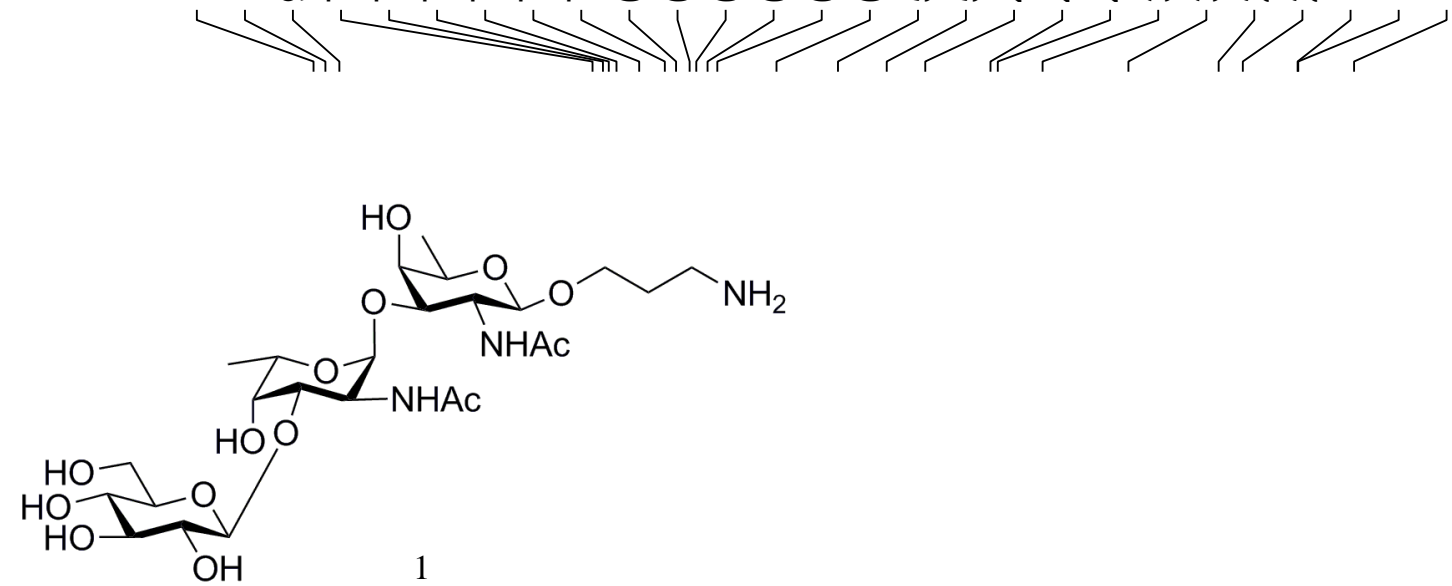

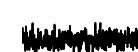

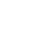

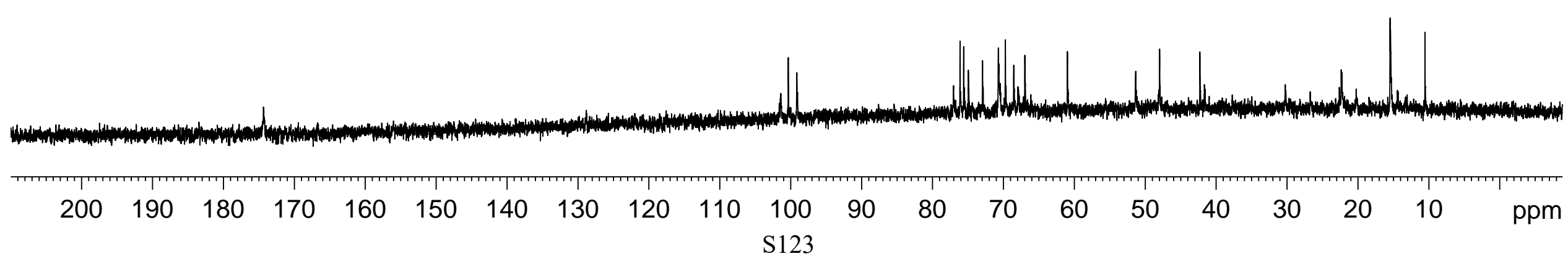




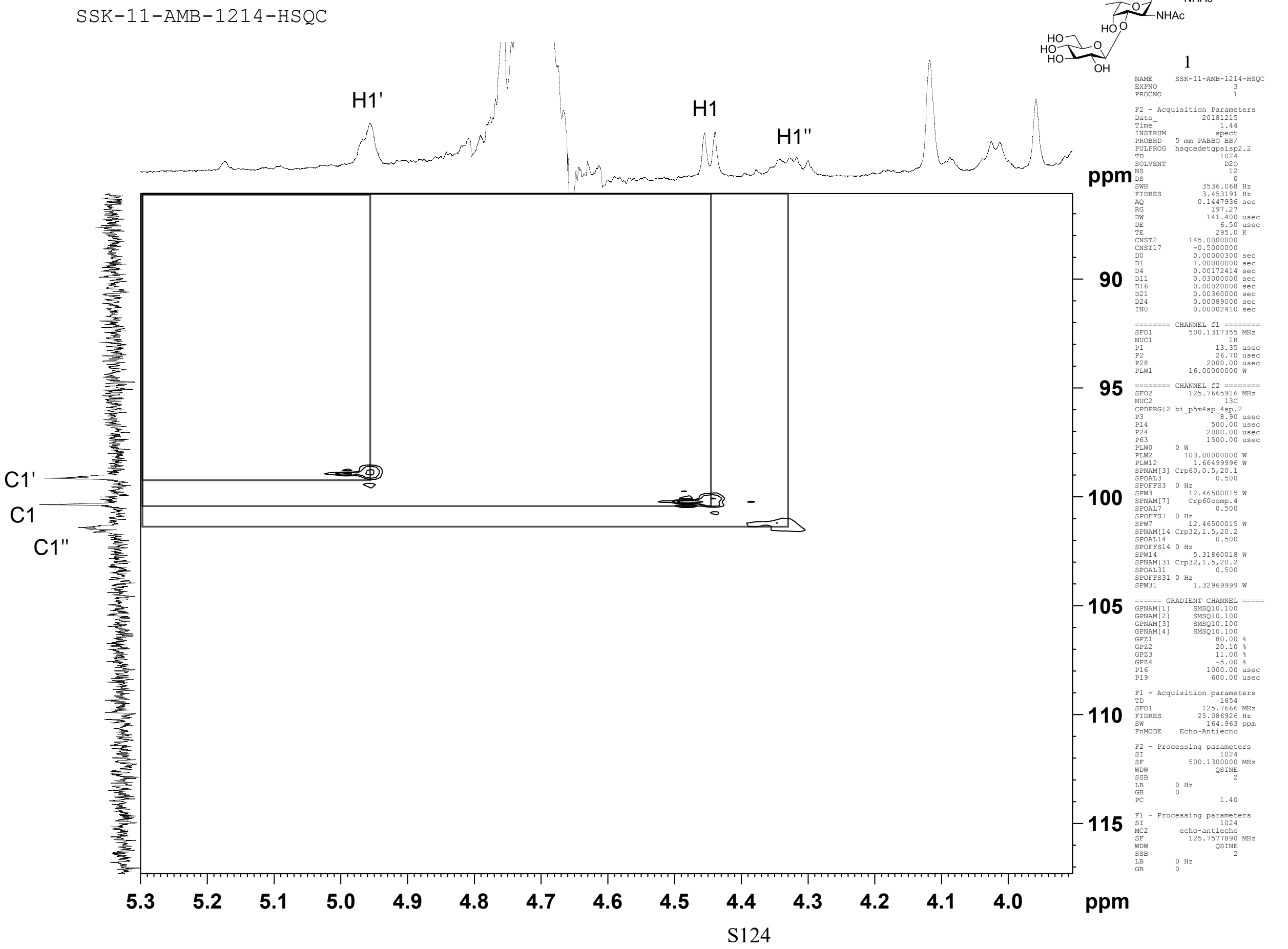




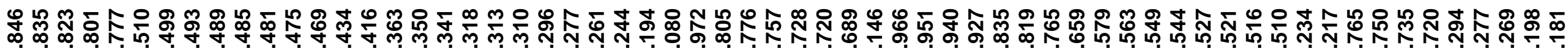

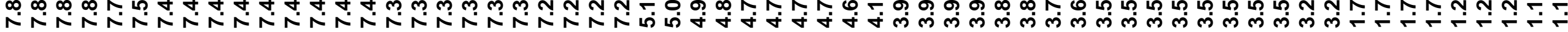
III

Current Data Parameters

EXPNO $\quad 1$

F2 - Acquisition Parameters

$\begin{array}{ll}\text { Date } & 20190801 \\ \text { Time } & 16.17\end{array}$

INSTRU

PROBHD 5 mm PABBO BB-

PULPROG $\quad \mathrm{zg} 30$

TD 54274

$\begin{array}{ll}\text { NS } & 8 \\ \text { DS } & 0\end{array}$

SWH $\quad 8223.685 \mathrm{~Hz}$

FIDRES $\quad 0.151522 \mathrm{~Hz}$

$\mathrm{AQ} \quad 3.2998593 \mathrm{sec}$

$\begin{array}{ll}\text { RG } & 161 \\ \text { DW } & 60.800 \text { usec }\end{array}$

6.50 usec

$\quad 1.00000000 \mathrm{sec}$

$=======$ CHANNEL $f 1$ =======

$\begin{array}{ll}\text { NUC1 } & 1 \mathrm{H} \\ \mathrm{P1} & 14.75 \text { usec }\end{array}$

PL1 $\quad-1.00 \mathrm{~dB}$

SFO1 $400.1324710 \mathrm{MH}$

F2 - Processing parameters

$\begin{array}{ll}\text { SI } & 32768 \\ \text { SF } & 400.1300095 \mathrm{MHz}\end{array}$

WDW 400.130M

$\begin{array}{lll}\text { SSB } & 0 & 0.30 \mathrm{~Hz}\end{array}$

$\begin{array}{lll}\text { GB } & 0 & 1.00\end{array}$

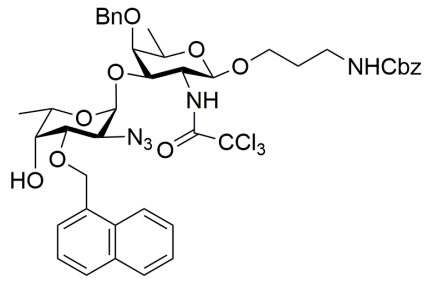

$30 \mathrm{c}$
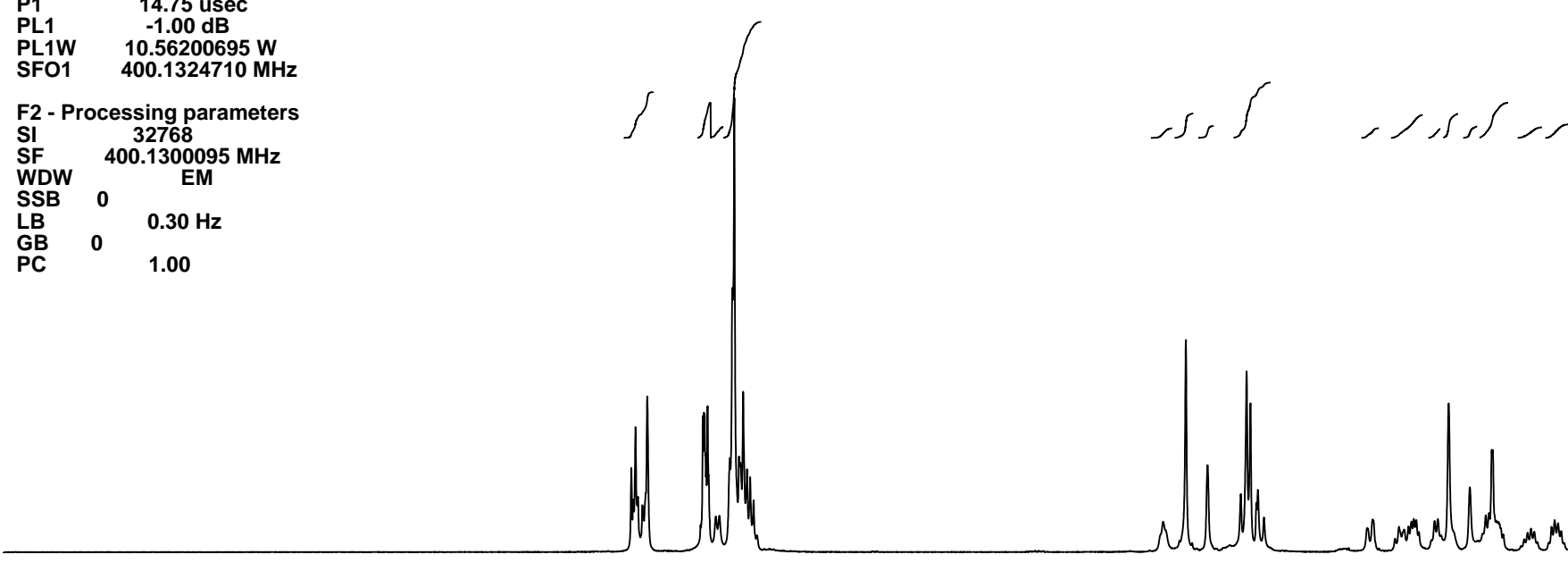

$\begin{array}{lll}10.5 & 10.0 \quad 9.5\end{array}$

9.0
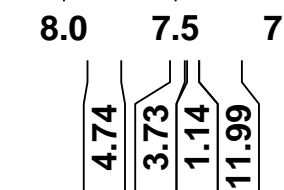

7.0

6.5

$6.0 \quad 5$

55.

4.5

$4.0 \quad 3.5$

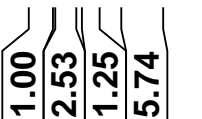
1) ل1 14 ㅇํㅇำ भิ S125

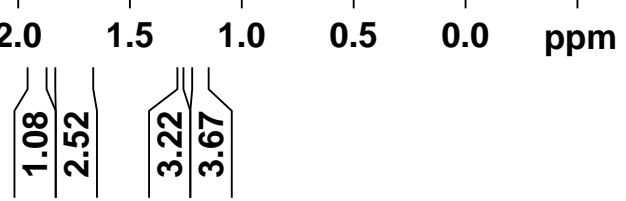


Current Data Parameters
NAME SSK-24-DR-408-13C EXPNO

F2 - Acquisition Parameters

$\begin{array}{ll}\text { Date } & 20190801 \\ \text { Time } & 13.24\end{array}$

INSTRUM $\begin{gathered}\text { spect } \\ \text { PROBHD } 5 \text { mm PABBO BB/ }\end{gathered}$

PULPROG ${ }_{65536}^{2 g} 30$

SOLVENT ${ }_{\text {NS }} \mathrm{CDCl}$

$\begin{array}{lc}\text { NS } & 52 \\ \text { DS } & 0 \\ \text { SWH } & 29761.904 \mathrm{~Hz}\end{array}$

FIDRES $\quad 0.454131 \mathrm{~Hz}$

$\begin{array}{ll}\text { AQ } & 1.1010048 \mathrm{sec} \\ \text { RG } & 197.27\end{array}$

$\begin{array}{ll}\text { DW } & 16.800 \text { usec } \\ \text { DE } & 6.50 \text { usec }\end{array}$

D1 $1.00000000 \mathrm{sec}$

D11 $0.03000000 \mathrm{se}$

======== CHANNEL $f 1=======$

SFO1 $125.7703637 \mathrm{MHz}$

$\begin{array}{lc}\text { P1 } & 8.90 \text { usec } \\ \text { PLW1 } & 103.00000000 \mathrm{~W}\end{array}$

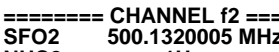

NUC2 $20.1320005 \mathrm{MHZ}$

$\begin{array}{lr}\text { CPDPRG } & \text { waltz16 } \\ \text { PCPD2 } & 80.00 \text { use }\end{array}$

PLW2 $16.00000000 \mathrm{~W}$

$\begin{array}{ll}\text { PLW12 } & 0.44556001 \mathrm{~W} \\ \text { PLW13 } & 0.22411001 \mathrm{~W}\end{array}$

F2 - Processing parameters

$\begin{array}{ll}\text { SI } & 32768 \\ \text { SF } & 125.7577890 \mathrm{MHz}\end{array}$

WDW 0 EM

LSB $0 \quad 1.00 \mathrm{~Hz}$

$\begin{array}{lll}\mathrm{GB} & 0 & 1.40\end{array}$

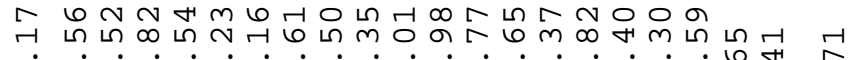

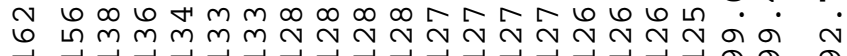

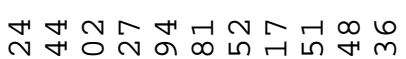

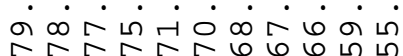

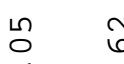

$\stackrel{\infty}{\stackrel{2}{N}}$

$-11111$

$\stackrel{\infty}{\infty} \stackrel{\infty}{N}$

ㅂ.

+

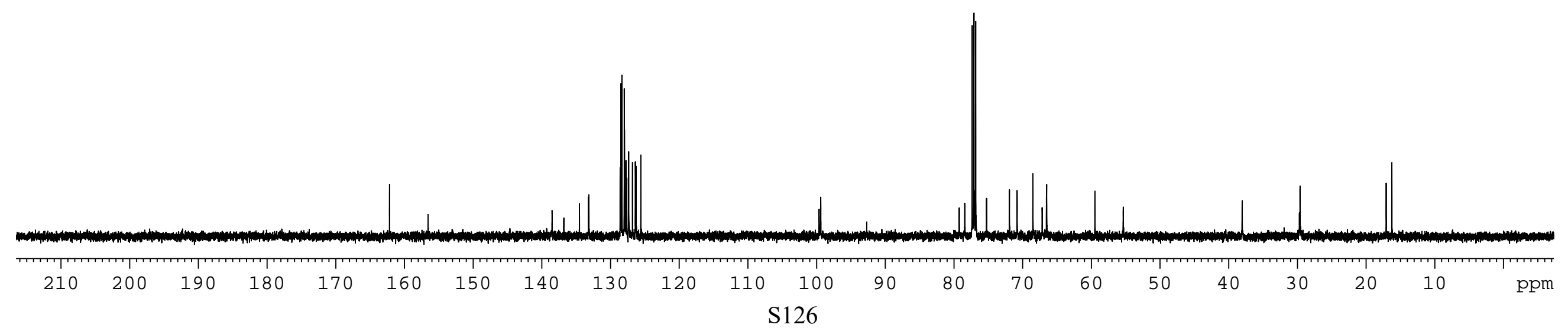




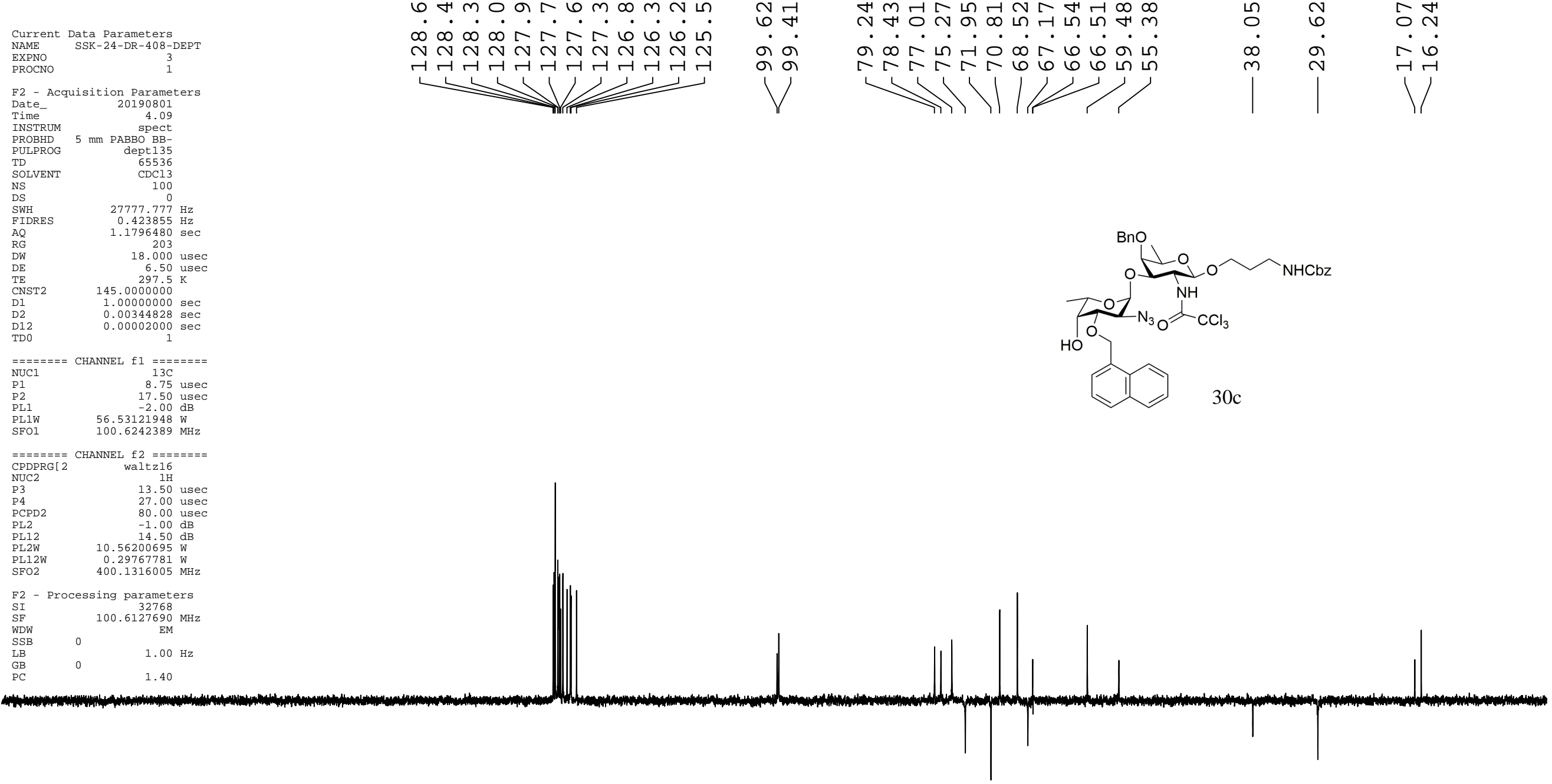

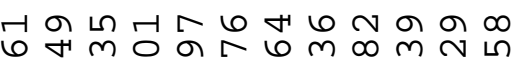

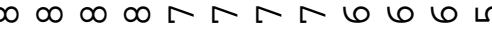
तन

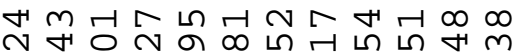

ำ ชิ

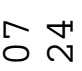

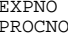

Parameter

Date spect
ABBO BB-

SDLVENT

$0.423555 \mathrm{~Hz}$

8.000 usec

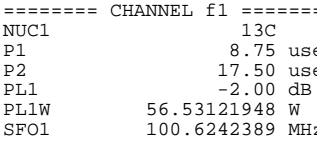

$=======$ CHANNEL $f 2=====$ 
ssk-24-dr-408-cosy

\section{CH2 OF $\mathrm{Cbz}$}

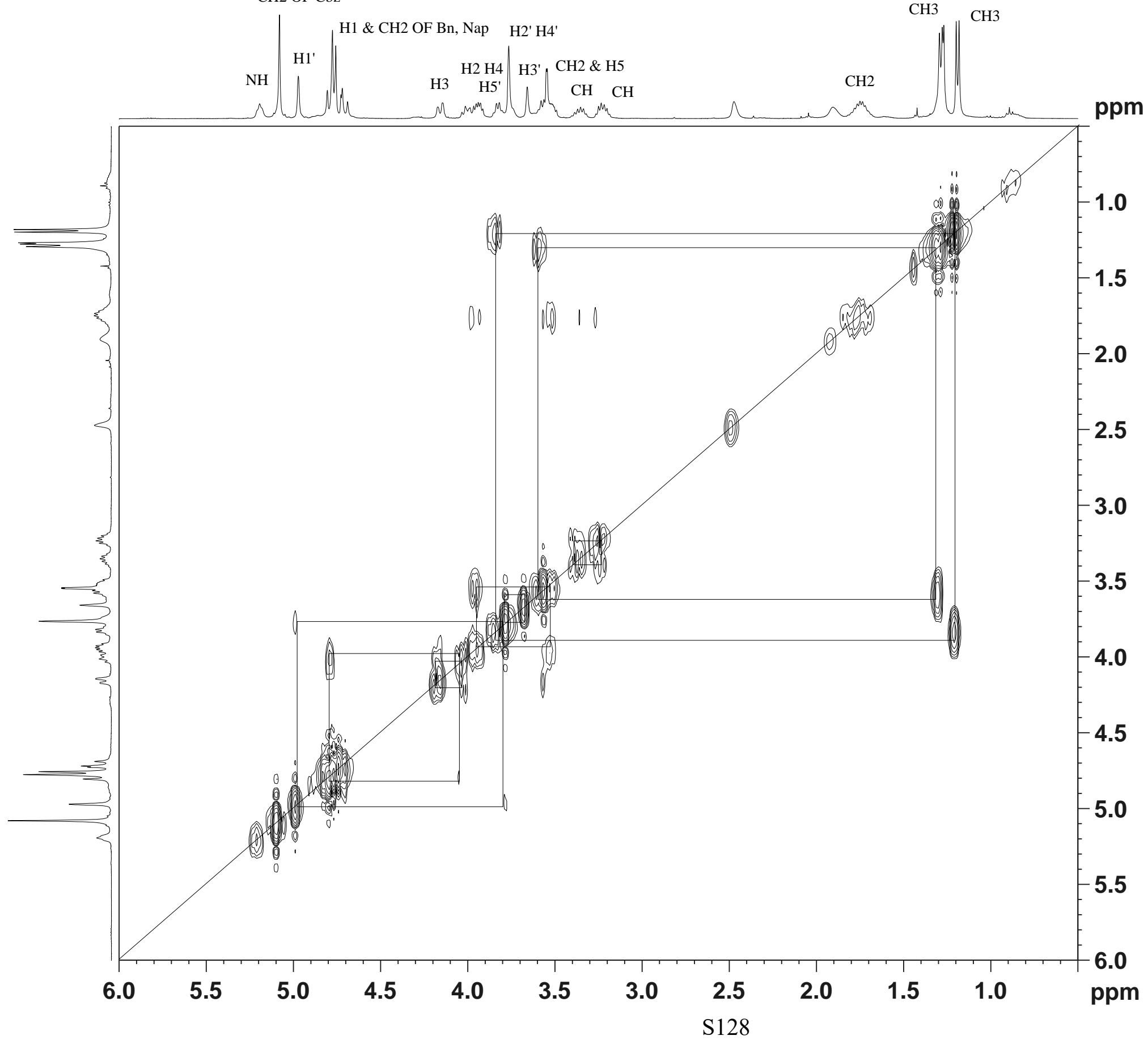

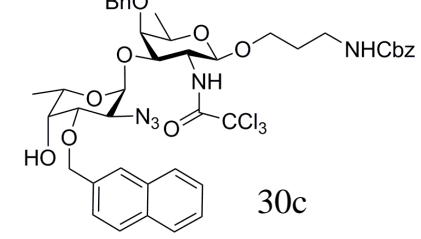

Current Data Parameters NAME
EXPNO

F2 - Acquisition Parameter

Date 20190801 PROBHD $5 \mathrm{~mm}$ PABBO BBPULPROG COsygpqf SOLVENT $\mathrm{CDCl} 13$
4 NS

$\begin{array}{ll}\text { DS } & \ominus \\ \text { SWH } & 5341.889 \mathrm{~Hz}\end{array}$ $\begin{array}{lr}\text { FIDRES } & 2.608340 \mathrm{~Hz} \\ \text { AO } & 0.1916928 \mathrm{sec}\end{array}$ $\begin{array}{lr}\text { RG } & 64 \\ \mathrm{DW} & 93.600 \mathrm{usec}\end{array}$ DE
TE
$296.90 \mathrm{Ku}$ DO $0.00000300 \mathrm{~K}$ $\begin{array}{ll}\text { D1 } & 1.000000000 \mathrm{sec} \\ \mathrm{D} 13 & 0.00000400 \mathrm{sec}\end{array}$ $\begin{array}{ll}\text { D13 } & 0.00000400 \mathrm{sec} \\ \text { D16 } & 0.00020000 \mathrm{sec}\end{array}$ $=======$ CHANNEL $f 1$ =======

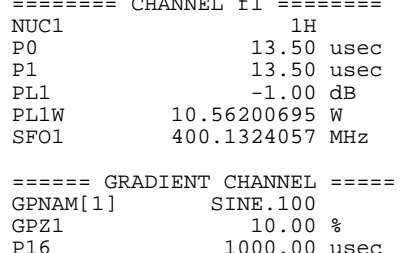
$\begin{array}{lr}\text { F1 - Acquisition parameters } \\ \text { TD } \\ \text { SF01 } & 400.1324 \mathrm{MHz}\end{array}$ $\begin{array}{lr}\text { SF01 } & 400.1324 \mathrm{MHz} \\ \text { FIDRES } & 159.459122 \mathrm{~Hz} \\ \text { SW } & 13.350 \mathrm{ppm}\end{array}$ F2 - Processing parameters $\begin{array}{lc}\text { SF } & 400.1300000 \\ \text { WDW } & \text { SINE }\end{array}$ $\begin{array}{llll}\text { SSB } & \ominus & \\ \text { LB } & \ominus & \\ \text { GB } & \ominus & \\ \text { PC } & & 1.40\end{array}$ F1 - Processing parameters $\begin{array}{lc}\text { SI } & 1024 \\ \text { MC2 } & \text { OF } \\ \text { SF } & 400.1300000 \mathrm{MHZ}\end{array}$ SF $400.1300000 \mathrm{MHZ}$ $\begin{array}{lll}\text { WSB } & \odot & \\ \text { LB } & \oplus \mathrm{Hz} & \end{array}$ 


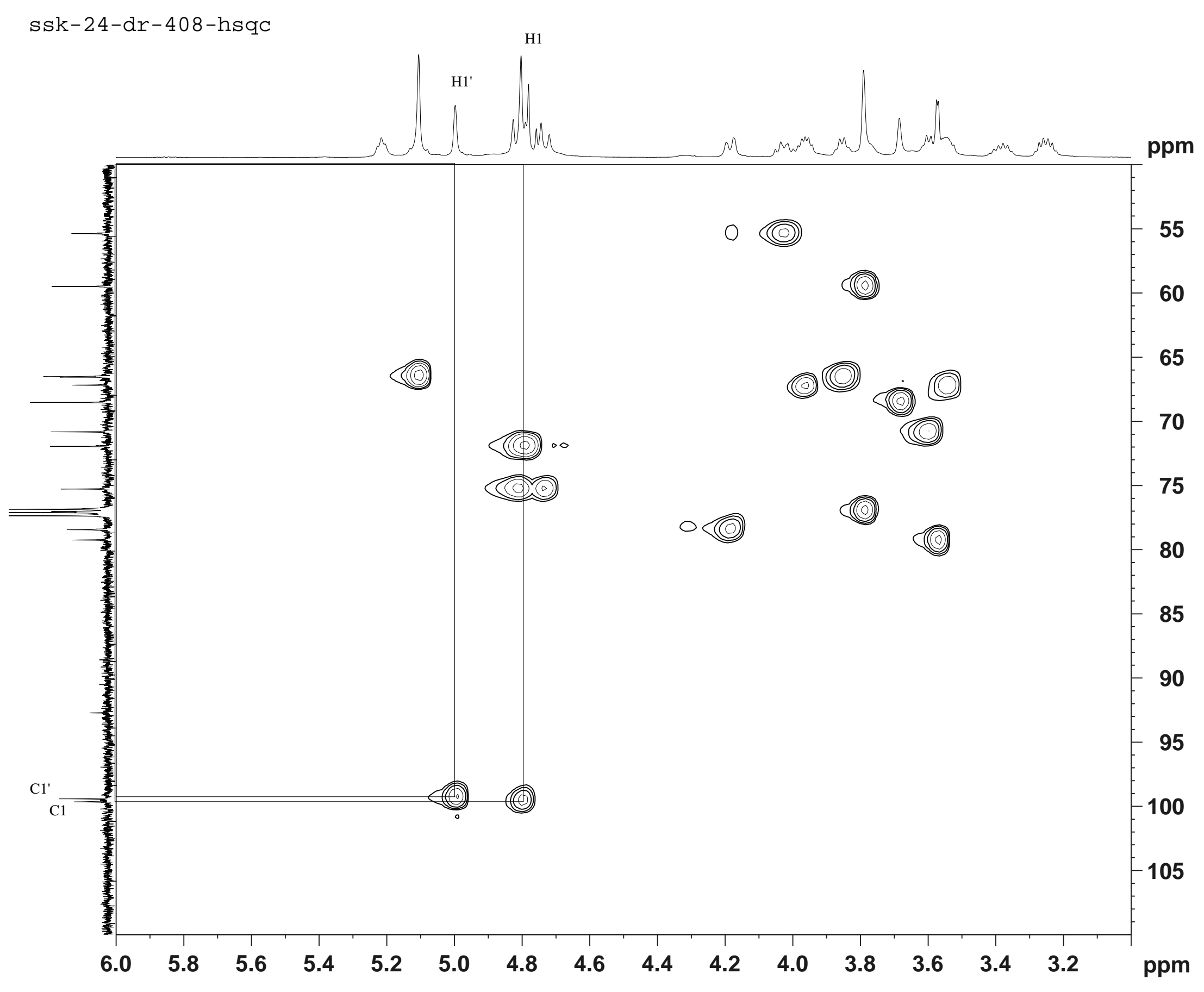

\section{BnO}

$\mathrm{NHCbz}_{\mathrm{NH}}$ $\mathrm{TT}_{\mathrm{O}}^{\mathrm{O}} \mathrm{Z}_{\mathrm{N}_{3} \mathrm{O}} \stackrel{\mathrm{NH}}{-} \mathrm{CCl}_{3}$ HO

30c

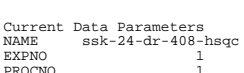

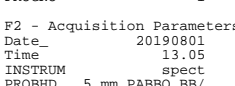

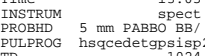

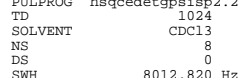

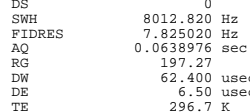

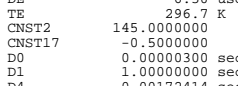

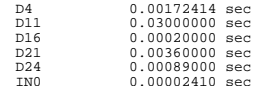

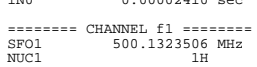

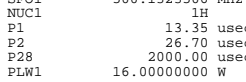

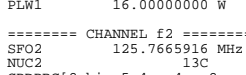

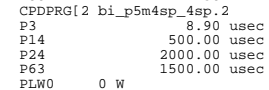

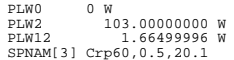

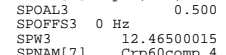

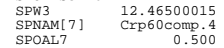

SPOFFS7
SP HZ HZ
SPNAM[14 $12.46500015 \mathrm{~W}$
SP $32,1.5,20.2$

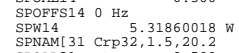

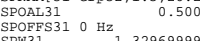

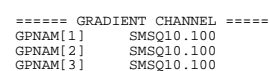

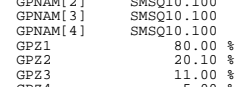

GP4
P16
P19

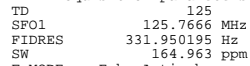

F2- Processing parameters

SI
SF
WDW
SSB

$\substack{\operatorname{SSB} \\ \text { LB } \\ \text { GB } \\ \text { PC }}$
PC

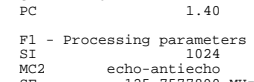

SF
WOW
SSB
LB 
Current Data Parameters
NAME SSK-24-DR-411-1H EXPNO 1

PROCNO

F2 - Acquisition Parameters

Time 201908

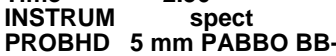

PULPROG ${ }_{54274}^{2930}$

SOLVENT 54274

NS

DS

SWH $\quad 002305$ H

$\begin{array}{ll}\text { FIDRES } & 0.151522 \mathrm{~Hz} \\ 3.2985993 \mathrm{seC} & \end{array}$

$\begin{array}{ll}\text { AQ } & 3.2998593 \mathrm{sec} \\ \text { RG } & 50.8\end{array}$

DW 60.800 usec

DE $\quad 6.50$ usec

D1 $1.00000000 \mathrm{sec}$

$=======$ CHANNEL $f 1$ =======

$\begin{array}{lll}\text { NUC1 } & 1 \mathrm{H} \\ \text { P1 } & 14.75 \text { usec }\end{array}$

$\begin{array}{ll}\text { PL1W } & 10.56200695 \mathrm{~W} \\ \text { SFO1 } & 400.1324710 \mathrm{MHz}\end{array}$

F2 - Processing parameters

$\begin{array}{ll}\text { SI } & 32768 \\ \text { SF } & 400.1300095 \mathrm{MHz}\end{array}$

WDW 400.130009

$\begin{array}{lll}\text { SSB } & 0 & 0.30 \mathrm{~Hz} \\ \text { LB } & 0 & \text { S }\end{array}$

$\begin{array}{lll}\mathrm{GB} & 0 & 1.00\end{array}$

\section{Tiin Tillinim}

\section{IIIIIII}

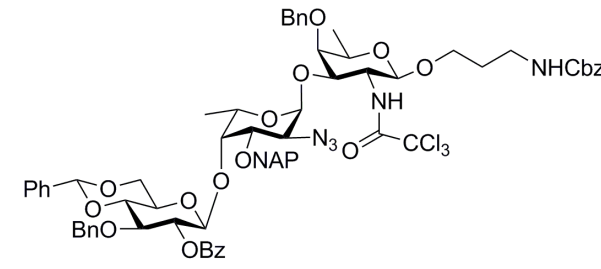

$4 \mathrm{c}$

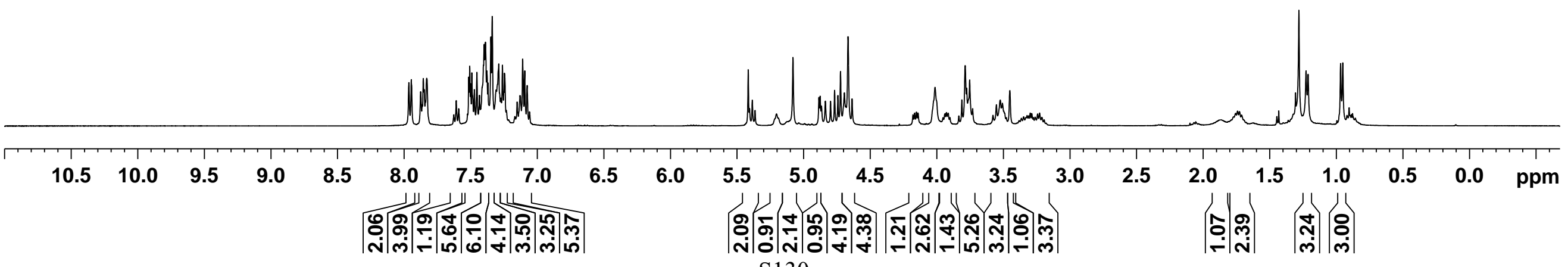
S130 


\section{SSK-24-DR-411-13C}

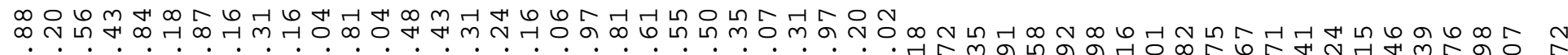

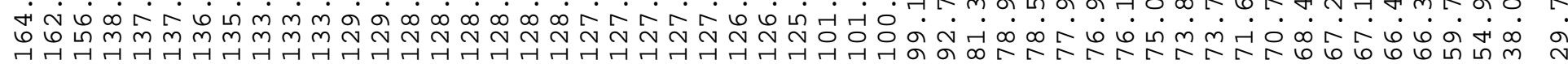

F2 - Acquisition Parameters

F2- Acquisition Param
Date
Time

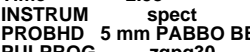

PULPROG ${ }_{655369}{ }_{\text {TD }}$

${ }_{\text {NS }}^{\text {SOLVNT }}{ }_{250}^{250} \mathrm{CDCl} 3$

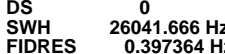

$\begin{array}{ll}\text { FDRES } & 0.0397364 \mathrm{~Hz} \\ \text { AQ } & 1.2582912 \mathrm{sec} \\ \text { RG } & 8922 \mathrm{ec}\end{array}$

$\begin{array}{ll}\mathrm{RG} & 1.212 \\ \mathrm{RW} & 81.20 \mathrm{usec} \\ \mathrm{DW} & 6.50 \mathrm{usec}\end{array}$

$\begin{array}{ll}6.50 \text { usec } \\ \text { TE } \\ \text { T1 } & 296.5 \mathrm{~K} \\ \text { D1 } & 1.00000000\end{array}$

$\begin{array}{ll}\text { D11 } & 1.00000000 \mathrm{sec} \\ \text { TDO } & 0.0300000 \mathrm{sec}\end{array}$

$=======$ CHANNEL $\mathrm{f1}$ =======

$\begin{array}{lr}\text { NUC1 } & 13 \mathrm{C} \\ \text { P1 } & 8.50 \mathrm{Csec} \\ \text { PL1 } & -2.00 \mathrm{~dB}\end{array}$

$\begin{array}{ll}\text { PL1W } & 56.53121948 \mathrm{~W} \\ \text { SFO1 } & 100.6238364 \mathrm{MHz}\end{array}$

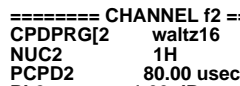

$\begin{array}{ll}\text { PL22 } & -1.00 \mathrm{~dB} \\ \text { PL12 } & 13.69 \mathrm{~dB} \\ \text { PL13 } & 14.50 \mathrm{~dB}\end{array}$

$\begin{array}{lr}\text { PL2W } & 10.56200695 \mathrm{~W} \\ \text { PL12W } & 0.35871249 \mathrm{~W}\end{array}$

PL13W $029767781 \mathrm{~W}$

Processing parameters

$\begin{array}{lc}\text { SI } & 32768 \\ \text { SF } & 100.6127690 \mathrm{MHz}\end{array}$

$\begin{array}{lll}\mathrm{SSB} & 0 & 1.00 \mathrm{~Hz}\end{array}$

$\begin{array}{lll}\mathrm{GB} & 0 & 1.00 \\ \mathrm{PC} & & 1.40\end{array}$
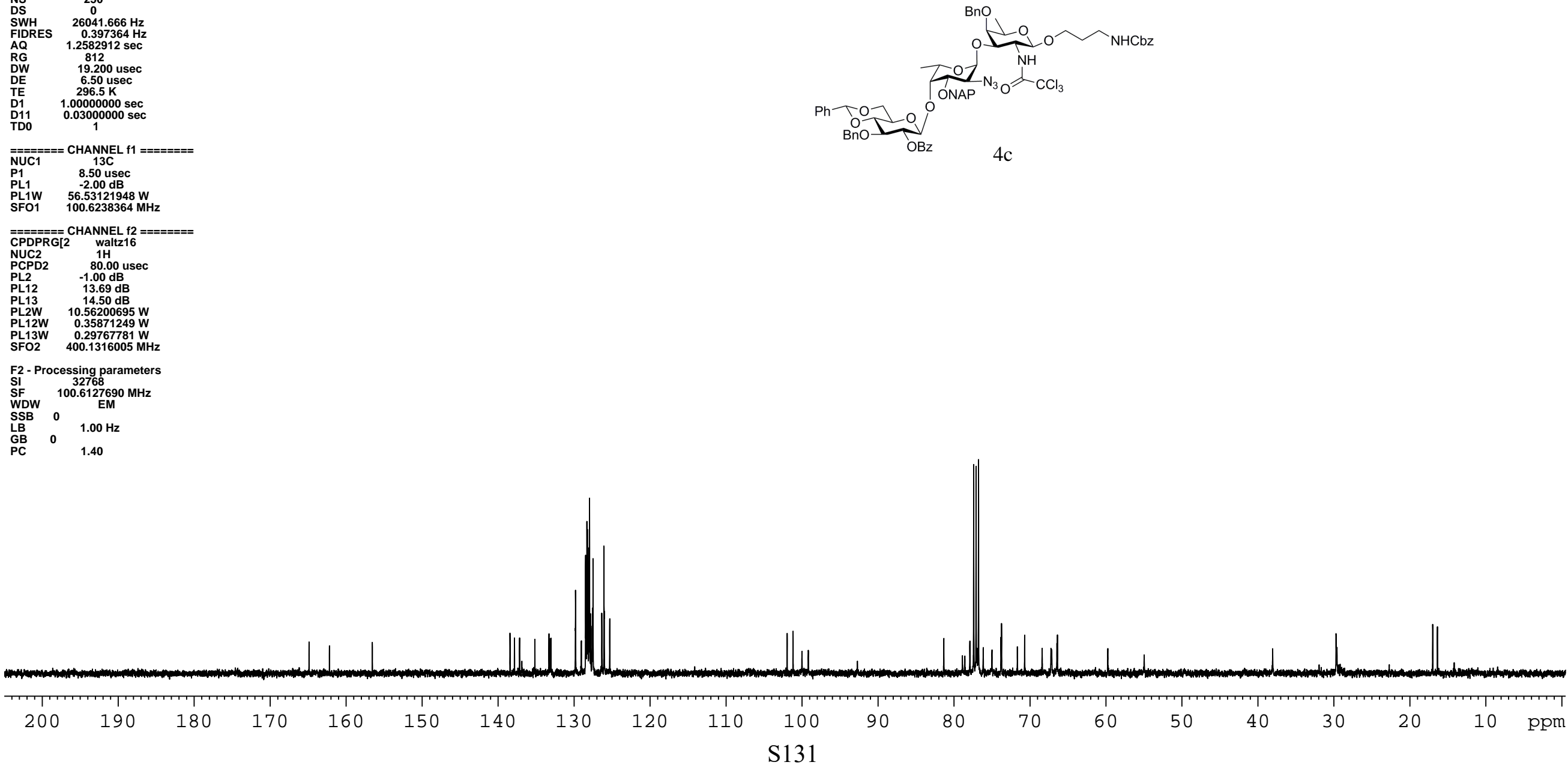


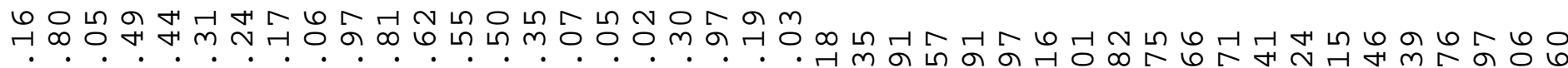

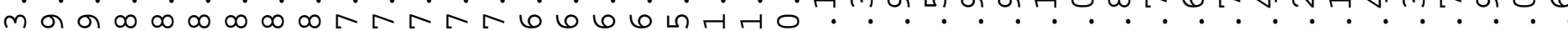

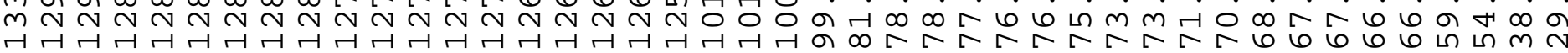

Current Data Parameters
NAME
SSK-24-DR-411-DEPT
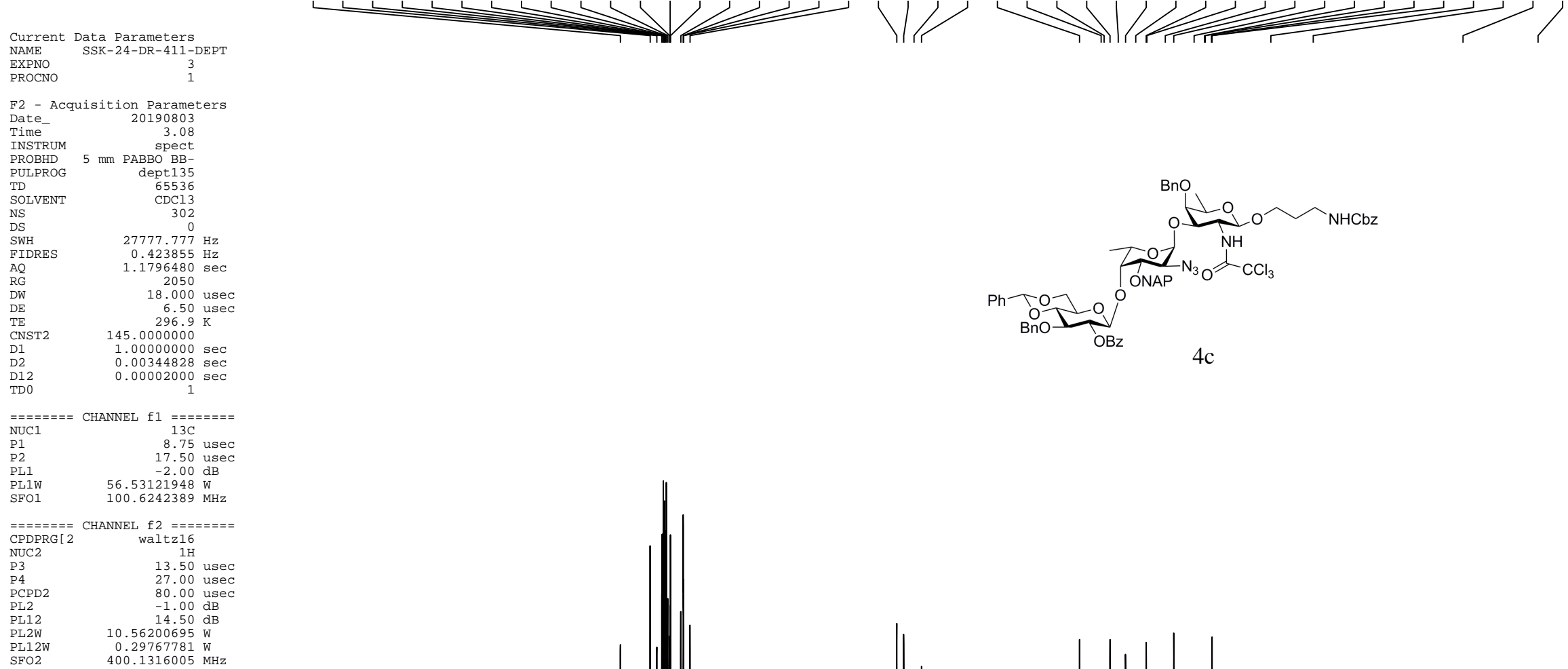

F2 - Processing paranters

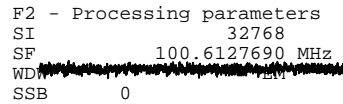

$1.00 \mathrm{~Hz}$

1.40

$4 \mathrm{c}$

1.40

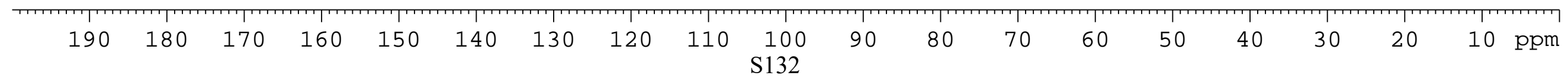




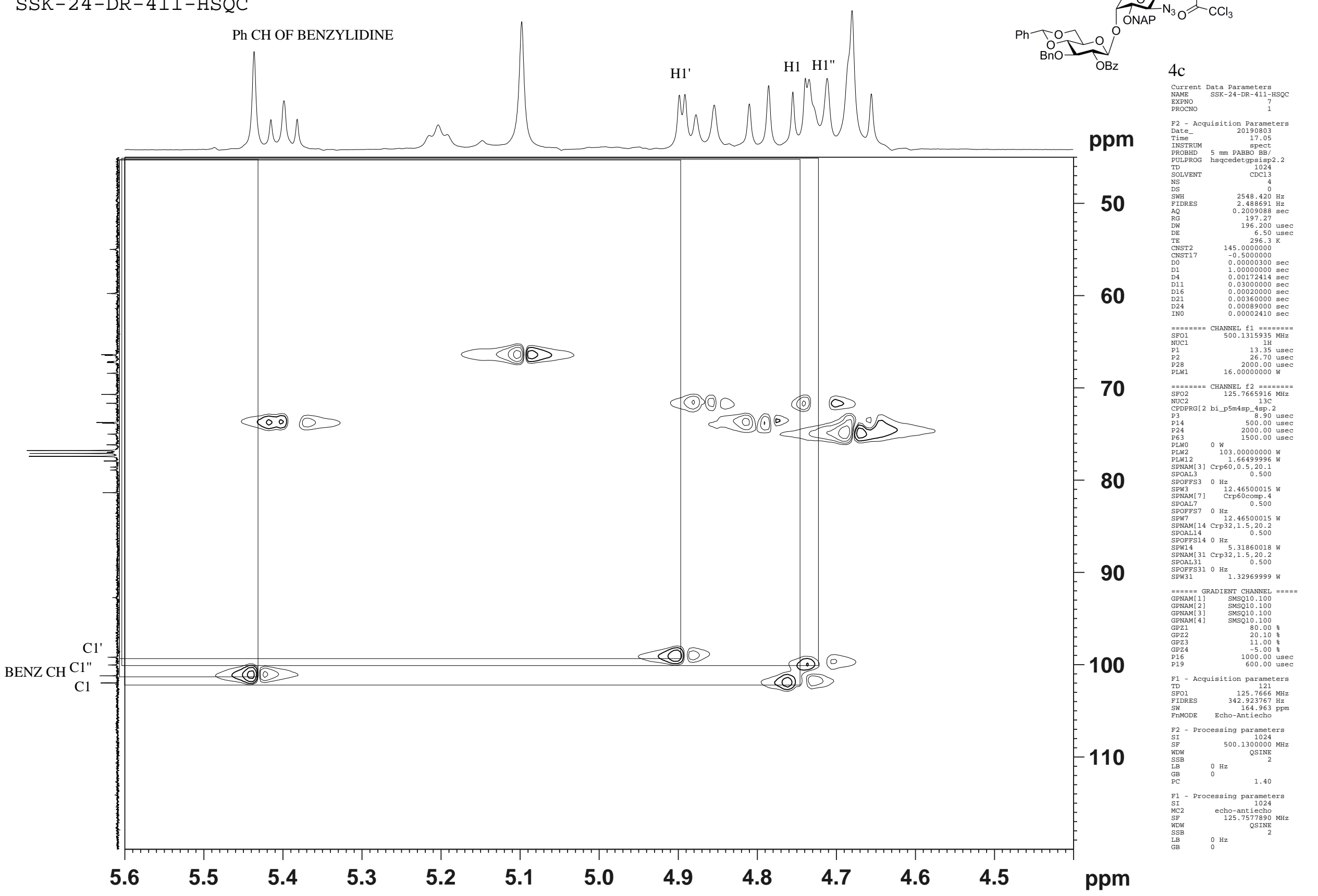




\section{SSK-24-DR-412-NEW-1H}

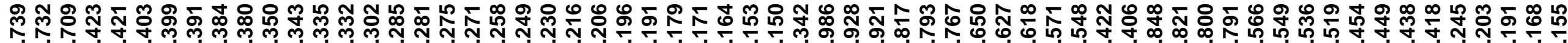

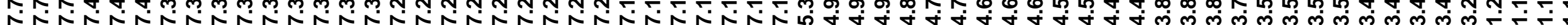

Current Data Parameters

NAME SSK-24-DR-412-NEW-1H

EXPNO 1

F2 - Acquisition Parameters

Date_ 20190809

Time

spect
PROBHD $5 \mathrm{~mm}$ PABBO BB

PULPROG ${ }_{6536} \mathrm{zg}^{20}$

NS $14{ }^{\mathrm{CDCl} 3}$

NS

$\begin{array}{lc}\text { DS } & 0 \\ \text { SWH } & 10000\end{array}$

FWH $\quad 10000.000 \mathrm{~Hz}$

AQ $\quad 3.2767999 \mathrm{sec}$

RG $\quad 30.72$

30.72
50.000 usec

6.50 usec

$\begin{array}{ll}\text { TE } & 296.6 \mathrm{~K} \\ \text { D1 } & 1.00000000 \mathrm{sec}\end{array}$

$\begin{array}{lr}\text { DDO } & 1.00000000 \\ & 1\end{array}$

$\begin{array}{ll}======= & \text { CHANNEL } f 1===== \\ \text { SFO1 } & 500.1330885 \mathrm{MHz} \\ \text { NUC1 } & 1 \mathrm{H} \\ \text { P1 } & 13.35 \text { usec }\end{array}$

P1 13.35 usec

F2 - Processing parameters

$\begin{array}{lc}\text { SI } & 65536 \\ \text { SF } & 500.1300582 \mathrm{MHz}\end{array}$

WDW $\quad 500.1300582$

SSB 0

LB $\quad 0.30 \mathrm{~Hz}$

PC $\quad 0 \quad 1.00$
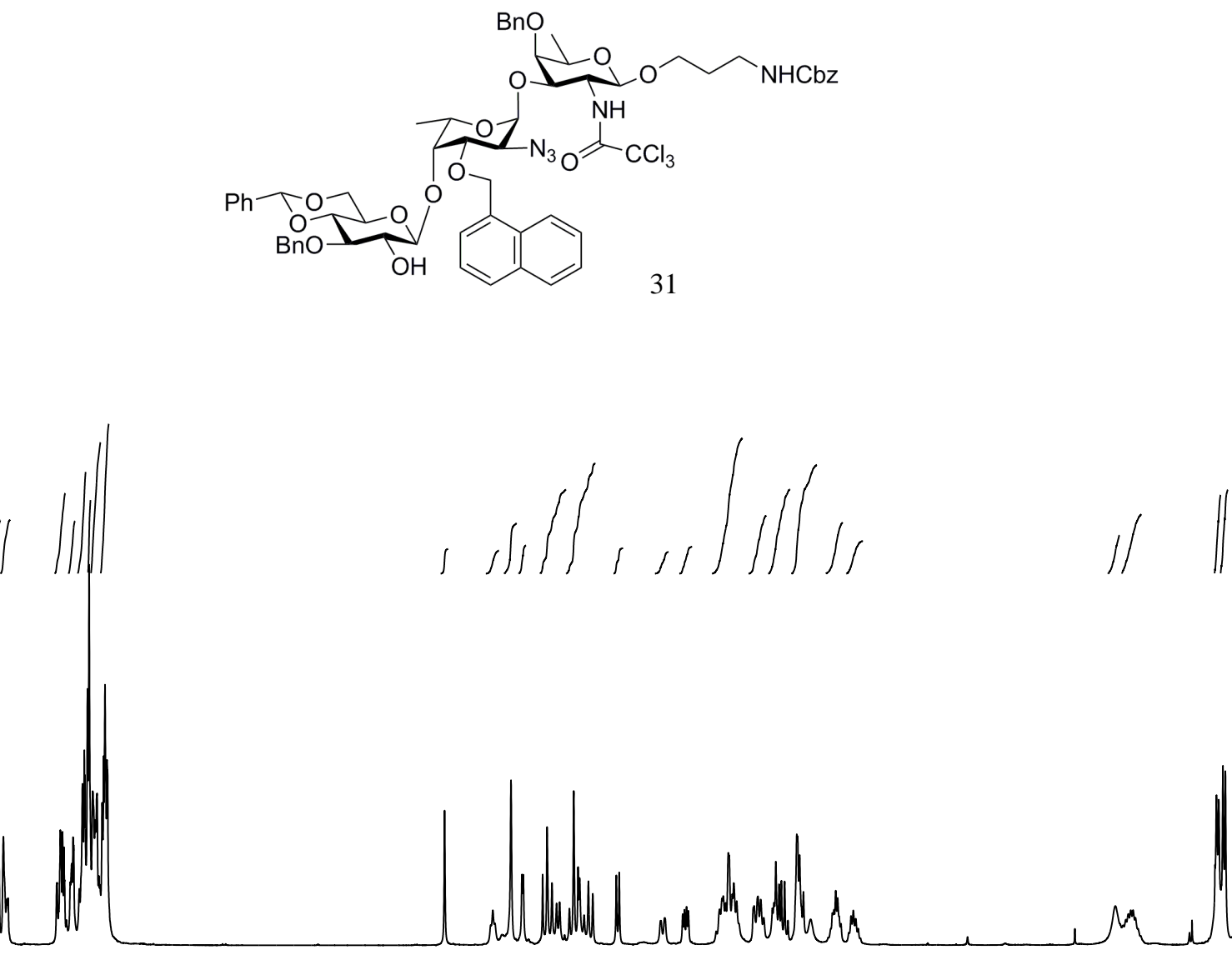

10.5

10.0

9.5

9.0

8.5

1.5

朵

6.5

6.0

5.5

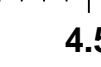

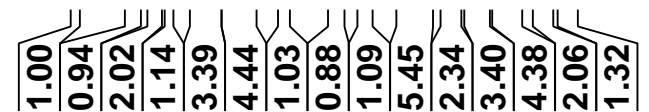

2.5

2.0

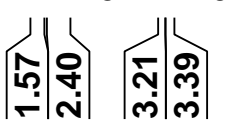

0.5

$0.0 \mathrm{ppm}$ S134 


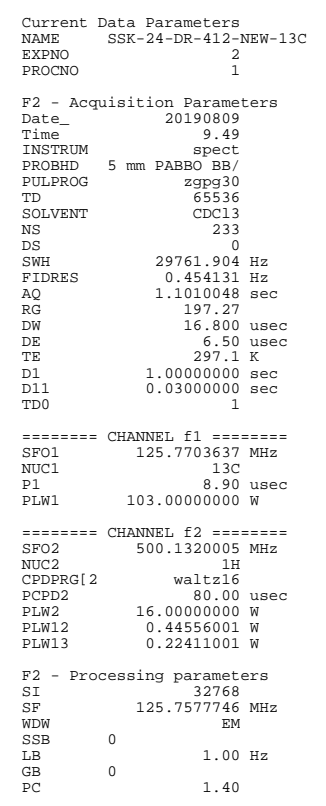

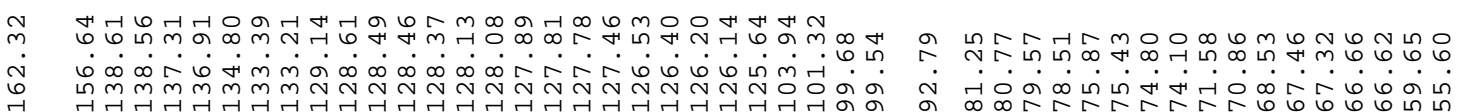

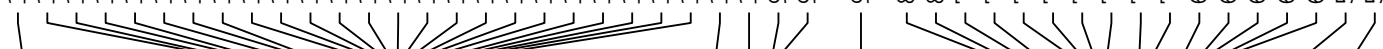

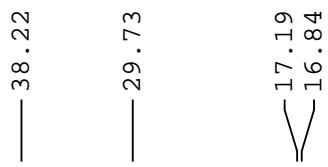
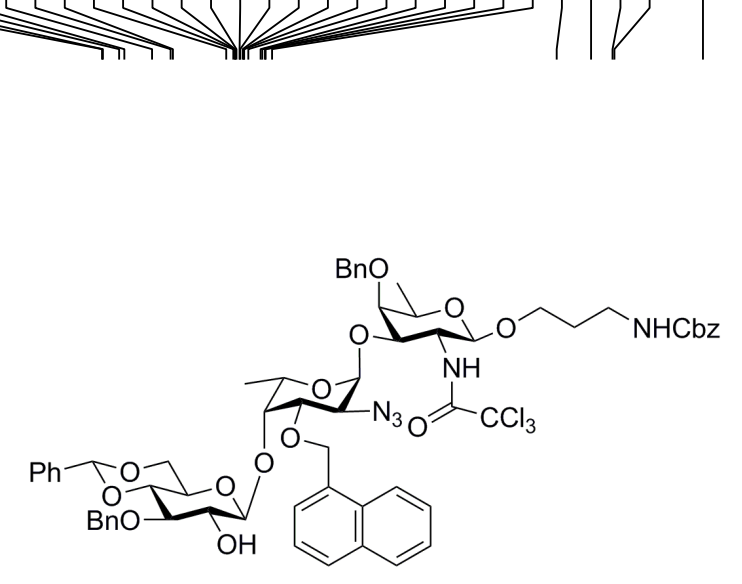

31

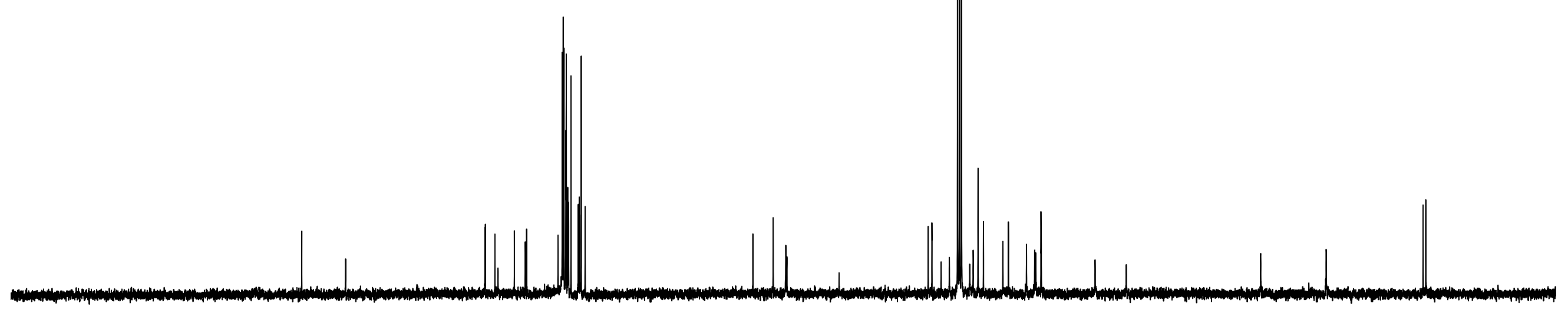


SSK - 24 - DR - 412 - NEW - DEPT1:

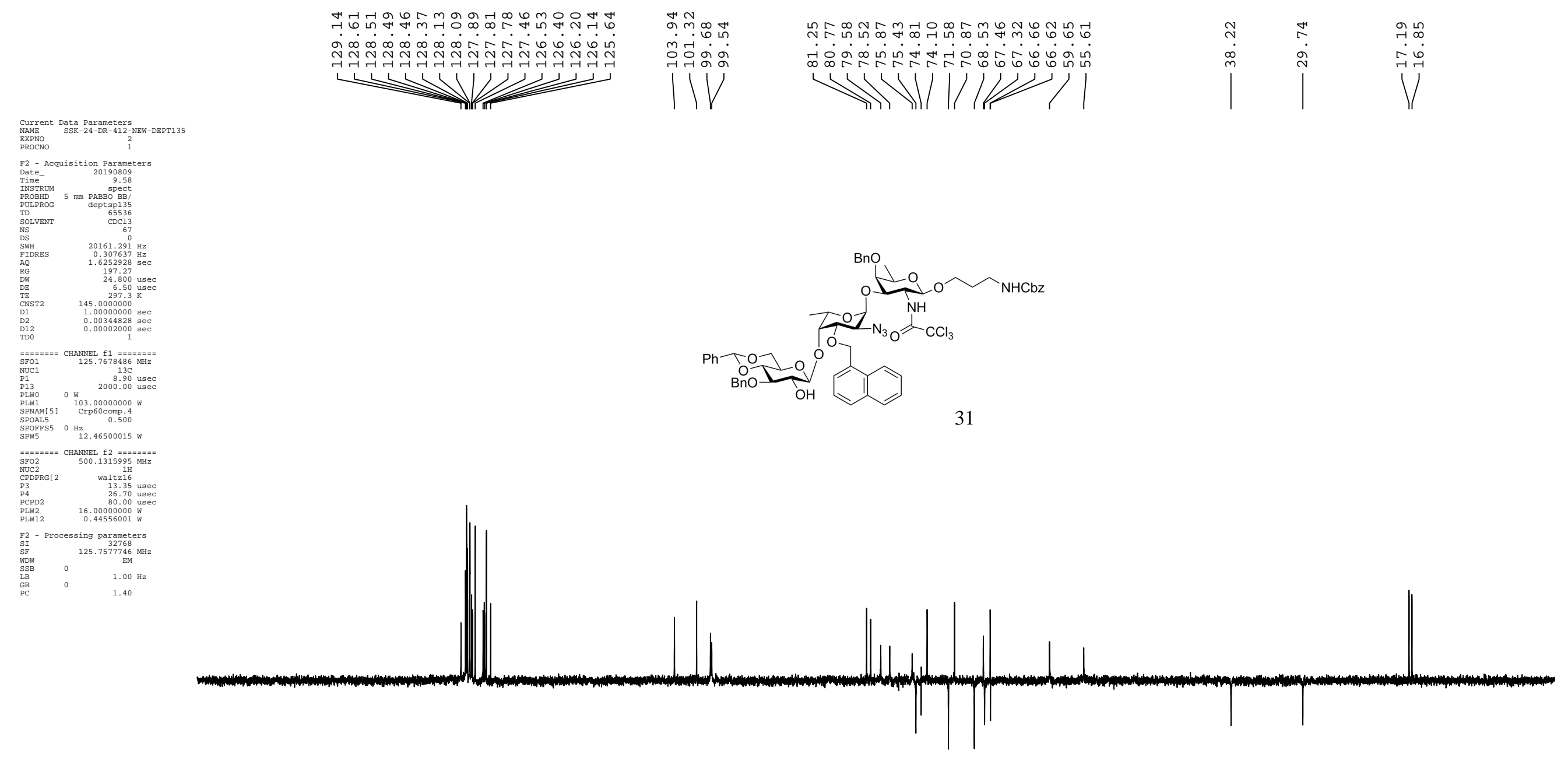




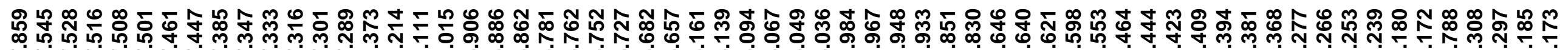

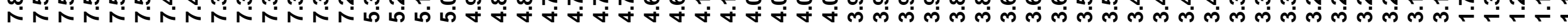

Current Data Parameters

EXPNO

PROCNO

F2 - Acquisition Parameters

$\begin{array}{lc}\text { Date } & 20190813 \\ \text { Time } & 18.34 \\ \text { INSTR } & \end{array}$

PROBHD $5 \mathrm{~mm}$ PABBO BBI

PULPROG $\mathrm{zg} 30$

TD

$\begin{array}{ll}\text { NS } & 22 \\ \text { DS } & 0\end{array}$

SWH

$\quad 10000.000 \mathrm{~Hz}$

AO $\quad 0.152588 \mathrm{~Hz}$

RG $\quad \begin{array}{ll}\text { A.2767999 sec } \\ \text { RG.37 }\end{array}$

DW $\quad 50.000$ usec

DE $\quad 6.50$ usec

D1 $\quad 1.00000000 \mathrm{sec}$

TD0 1

$=======$ CHANNEL $f 1$ =======

NUC1 500.133

P1 13.35 usec

PLW1 $\quad \begin{aligned} & 16.00000000 \mathrm{~W} \\ & \text { PLW }\end{aligned}$

F2 - Processing parameters

$\begin{array}{ll}\text { SI } & 65536 \\ \text { SF } & 500.1300000 \\ \text { MHz }\end{array}$

WDW EM

$\begin{array}{lll}\text { SSB } & 0 & 0.30 \mathrm{~Hz}\end{array}$

$\begin{array}{lll}\mathrm{GB} & 0 & \mathbf{P C}\end{array}$
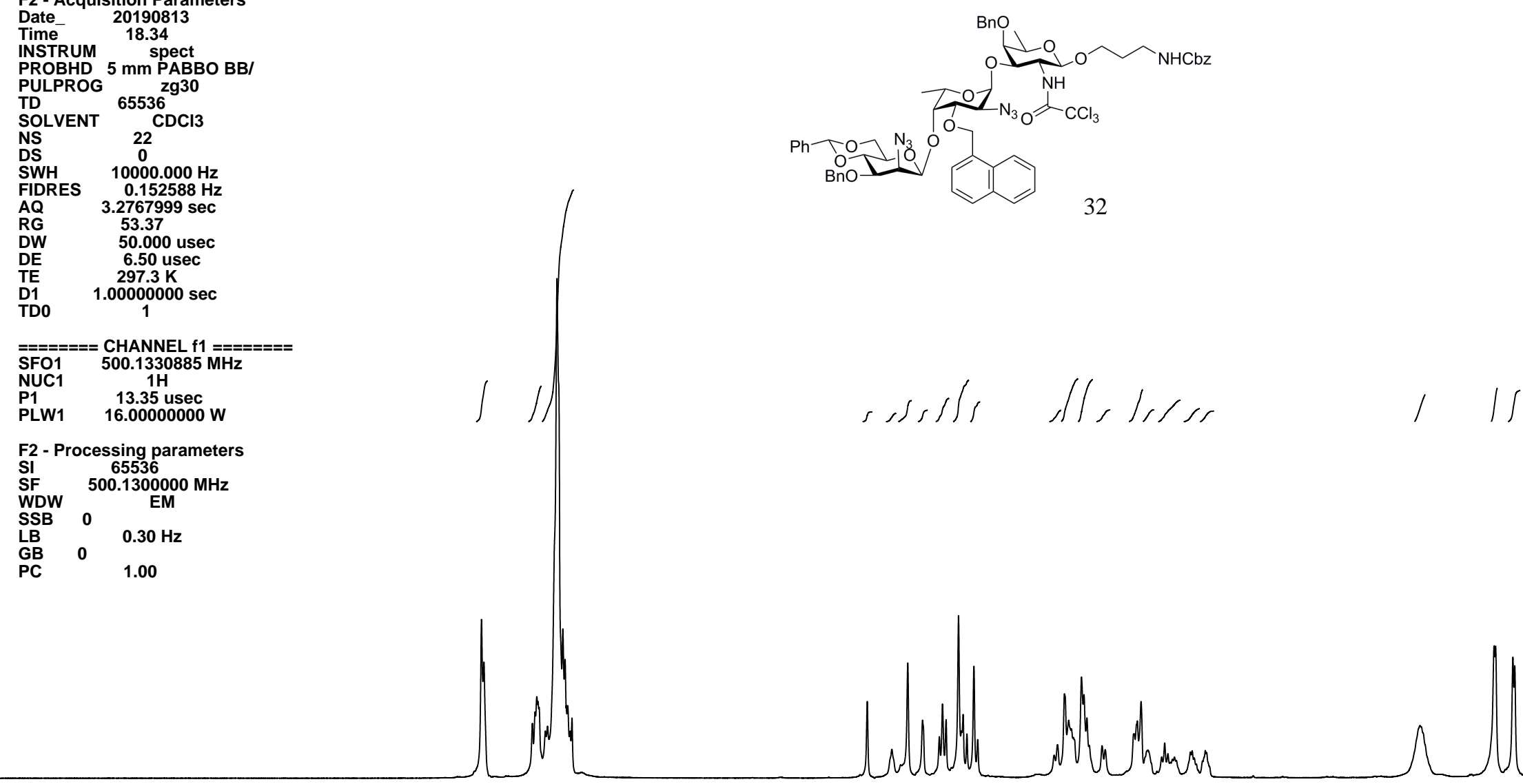

10.510 .0

9.0

8.5

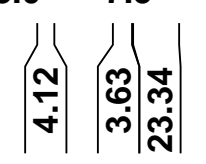

7.0

6.5
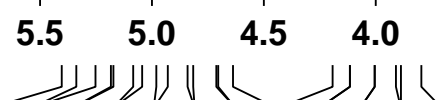

ర:

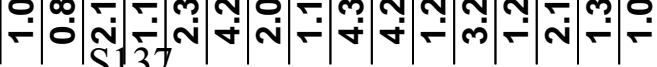

2.5

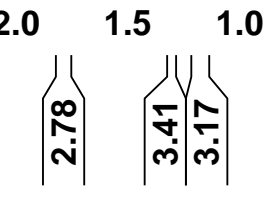




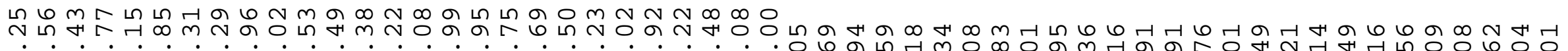
نón்

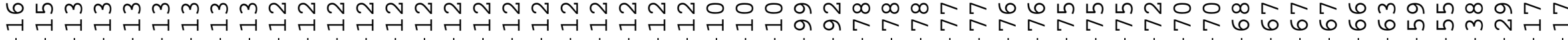
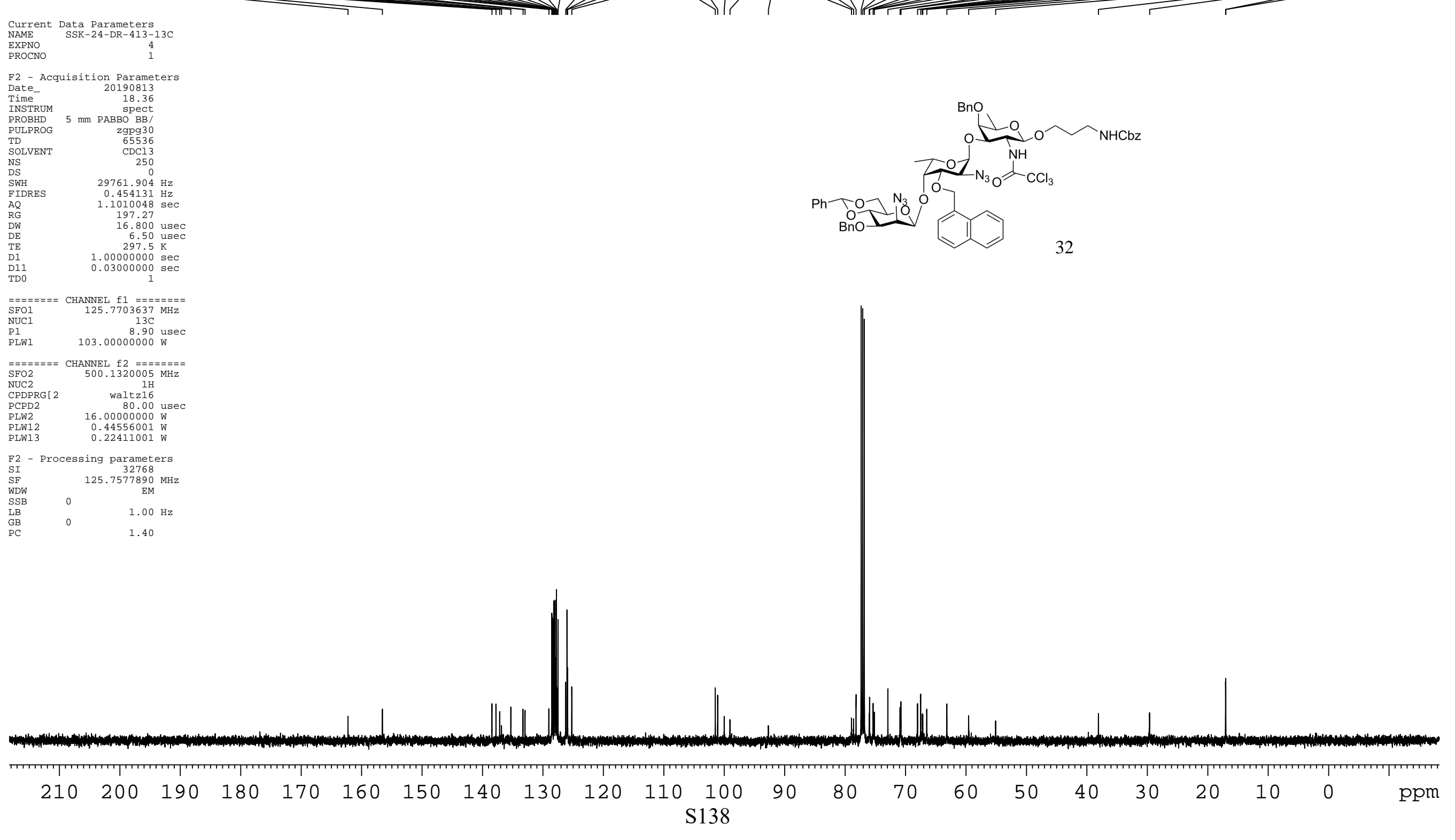


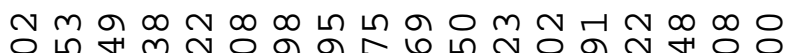

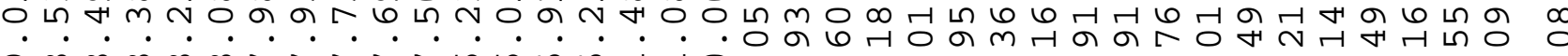
จำ 각 각ㄱㄱㄱㄱㄱㄱㄱㄱㄱㄱㄱㄱㄱ

Current Data Parameters
NAME SSK-24-DR-413-DEPT

NAME $\quad$ SSK-24-DR-413-DEP

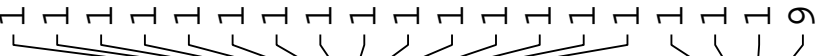

5
1
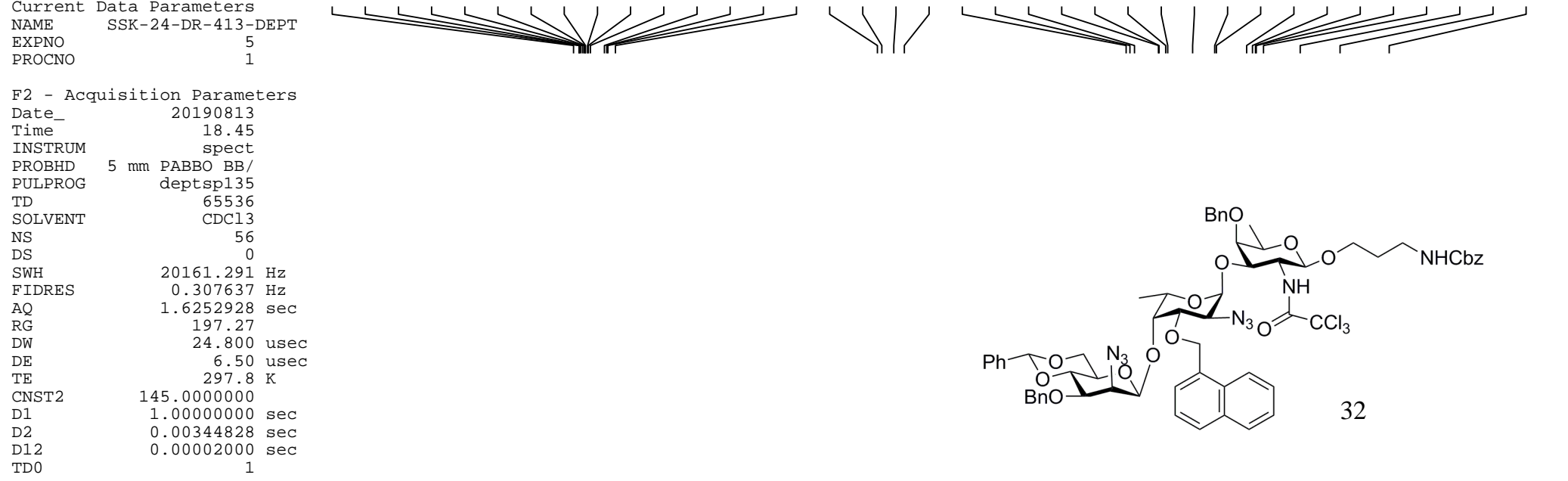

$=======$ CHANNEL $\mathrm{f} 1========$
SF01 $125.7678486 \mathrm{MHz}$

$\begin{array}{lr}\text { SF01 } & 125.7678486 \mathrm{MHz} \\ \text { NUC1 } & 13 \mathrm{C} \\ \text { P1 } & 8.90 \mathrm{usec} \\ \text { P13 } & 2000.00 \mathrm{usec}\end{array}$

PLW0 $\odot \mathrm{W}$. $103.000000000 \mathrm{~W}$

$\begin{array}{lr}\text { PLW1 } & 103.000000000 \\ \text { SPNAM [5] } & \text { Crp600omp.4 }\end{array}$

$\begin{array}{lcc}\text { SPOFFS5 } & \odot \mathrm{Hz} & 0.500 \\ \text { SPW5 } & 12.46500015 \mathrm{~W}\end{array}$

$=======$ CHANNEL $\mathrm{f} 2$ =-

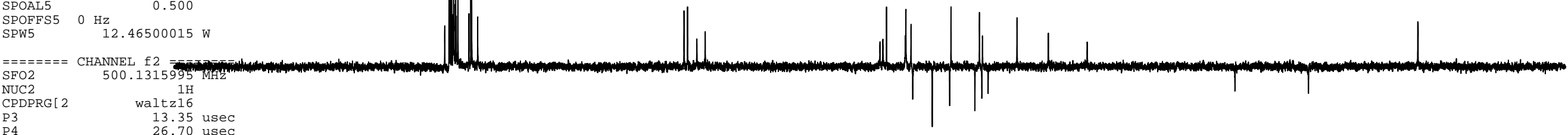

CPDPRG [2 waltz16

$\begin{array}{lr}\text { P3 } & 13.35 \text { usec } \\ \text { P4 } & 26.70\end{array}$

$\begin{array}{ll}\text { PCPD2 } & \text { 80.00 usec }\end{array}$

$\begin{array}{lr}\text { PLW2 } & 16.00000000 \mathrm{~W} \\ \text { PLW12 } & 0.44556001 \mathrm{~W}\end{array}$

\begin{tabular}{lcc} 
F2 & - Processing parameters \\
SI & 32768 \\
SF & \multicolumn{1}{c}{$\begin{array}{c}\text { 125.7577890 MHz } \\
\text { WDW }\end{array}$} & EM \\
SSB & 0 & $1.00 \mathrm{~Hz}$ \\
LB & 0 & 1.40 \\
GB & 0 &
\end{tabular}

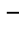


H1

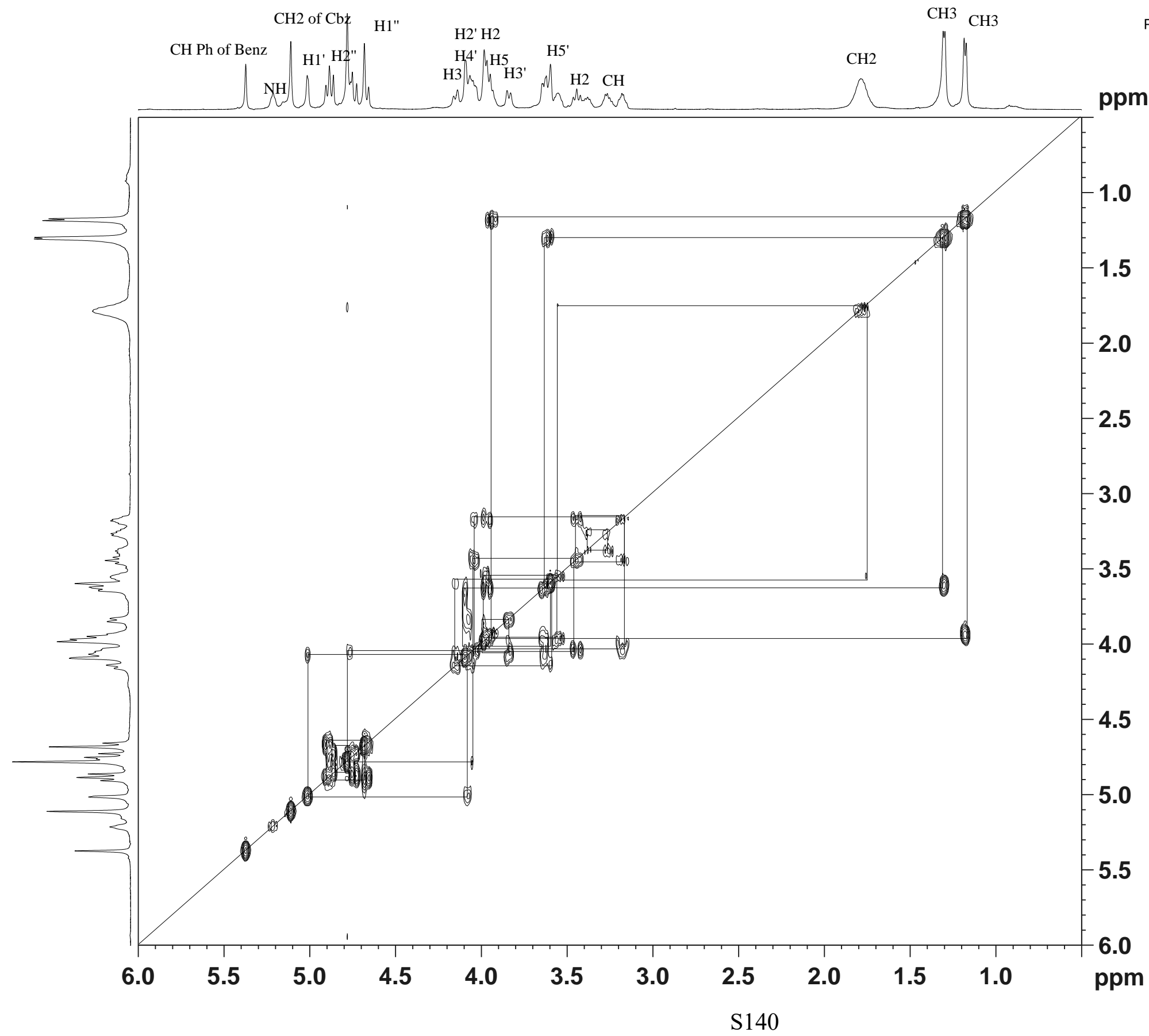

$\mathrm{Ph}$

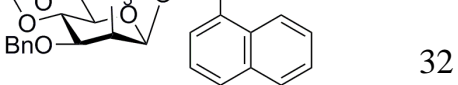

Current Data Parameters
NAME
SSK-24-DR-413-coSY EXPNO
PROCNO

F2 - Acquisition Parameters

Date ${ }_{-}$

20190813
Time

PROBHD $5 \mathrm{~mm}$ PABBO BB/

PULPROG cosygpppqf

SOLVENT

CDC13

NS
DS

$\begin{array}{lr}\text { DS } & \stackrel{4}{\ominus} \\ \text { SWH } & 4325.259 \mathrm{~Hz}\end{array}$

$\begin{array}{lr}\text { SWH } & 4325.259 \mathrm{~Hz} \\ \text { FIDRES } & 2.111943 \mathrm{~Hz} \\ \text { ADR } & 0.2367488 \mathrm{sec}\end{array}$

$\begin{array}{lr}\text { AQ } & 0.2367488 \mathrm{sec} \\ \text { RG } & 61.42\end{array}$

$\begin{array}{lr}\text { DW } & 115.600 \mathrm{usec} \\ \text { DE } & 6.50 \mathrm{usec} \\ \text { TE } & 297.5 \mathrm{~K}\end{array}$

$\begin{array}{lr}\text { TE } & 297.5 \mathrm{~K} \\ \text { DO } & 0.0000300 \mathrm{sec}\end{array}$

$\begin{array}{ll}\text { D1 } & 1.00000000 \text { sec } \\ \text { D11 } & 0.03000000 \text { sec } \\ \text { D12 } & 0.00002000 \text { sec } \\ \text { D13 } & 0.0000000 \text { Se }\end{array}$

D11
D12
D13

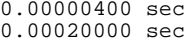

0.00023120 sec

$=======$
CHANNEL $\mathrm{f} 1========$
SF01
$500.1321690 \mathrm{MHz}$

$\begin{array}{lr}\text { SF01 } & 500.1321690 \mathrm{MHz} \\ \text { NUC1 } & 1 \mathrm{H} \\ \text { P0 } & 13.35 \text { usec } \\ \text { P1 } & 13.35 \text { usec } \\ \text { P17 } & 5000.00 \text { usec } \\ \text { PLW1 } & 16.00000000 \mathrm{~W} \\ \text { PL10 } & 3.16840005 \mathrm{~W}\end{array}$

$\begin{array}{lr}\text { PLW1 } & 16.00000000 \mathrm{~W} \\ \text { PLW10 } & 3.16840005 \mathrm{~W}\end{array}$

$======$ GRADIENT CHANNEL $=$

GPNAM [1] SMSQ10.100

$\begin{array}{lc}\text { P16 } & 10.00 \% \\ \text { P16 } & 1000.00 \text { usec }\end{array}$

F1 - Acquisition parameter

128

$\begin{array}{lr}\text { SFO1 } & 500.1322 \mathrm{MHz} \\ \text { FIDRES } & 67.582176 \mathrm{~Hz}\end{array}$

$\begin{array}{lr}\text { SW } & \text { FnMODE } \\ & \text { QF }\end{array}$

F2 - Processing parameters

$\begin{array}{ll}\text { SI } & 1024 \\ \text { SF } & 500.1300000 \mathrm{MHZ}\end{array}$

WDW $\odot$

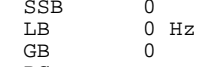

PC $\quad 1.40$

F1 - Processing parameters

$\begin{array}{ll}\text { SI } & 1024 \\ \text { MC2 } & \text { QF }\end{array}$

$\begin{array}{lc}\text { MC2 } & \text { QF } \\ \text { SF } & 500.1300000 \\ \text { WDW } & \text { MHz }\end{array}$

WDW
SSB
LB

$\begin{array}{lll}\text { LB } & 0 \mathrm{~Hz} \\ \mathrm{~GB} & 0\end{array}$ 


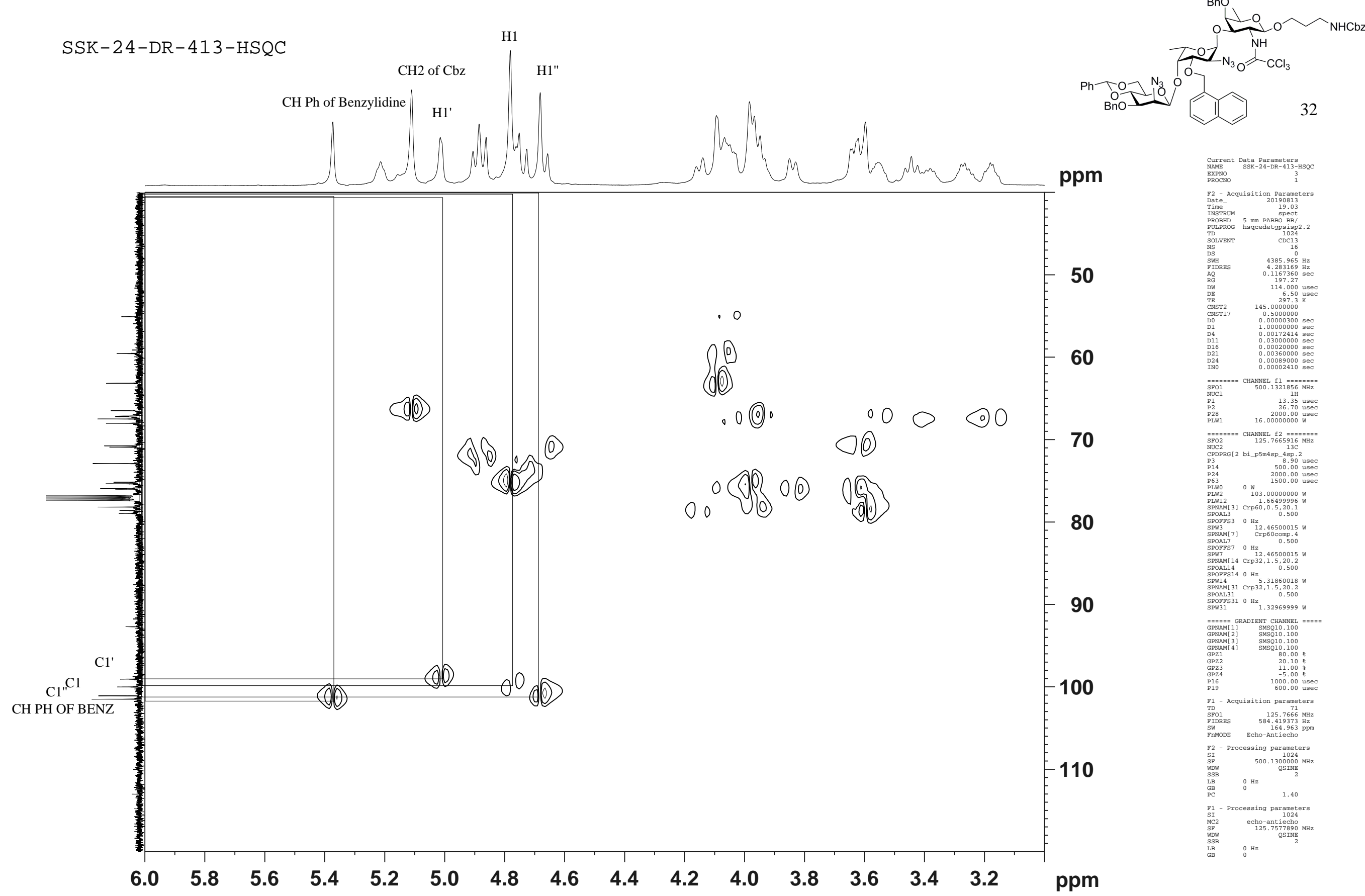




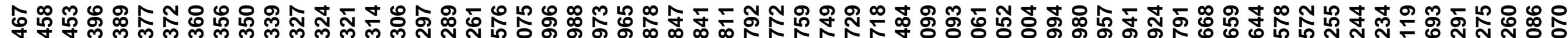 ヘ}

Current Data Parameters

$\begin{array}{ll}\text { EXPNO } & 13 \\ \text { PROCNO } & 1\end{array}$

F2 - Acquisition Parameters

20190824

INSTRUM

$$
\begin{aligned}
& 19.29 \\
& \text {. }
\end{aligned}
$$

PROBHD $5 \mathrm{~mm}$ PABBO BB

PULPROG $2 \mathrm{zg} 30$

TD

NS $25{ }^{20 C l 3}$

SWH $\quad 8223.685 \mathrm{~Hz}$

FIDRES $\quad 0.151522 \mathrm{~Hz}$

$\begin{array}{ll}\text { AQ } & 3.2998593 \mathrm{sec} \\ \text { RG } & 161\end{array}$

$\begin{array}{ll}\text { RG } & 161 \\ \text { DW } & 60.800 \text { usec }\end{array}$

DE 6.50 usec

$\begin{array}{ll}\text { TE } & 296.7 \mathrm{~K} \\ \text { D1 } & 1.00000000 \mathrm{sec}\end{array}$

$\begin{array}{lc}======= & \text { CHANNEL } \mathrm{f} 1 \\ \text { NUC1 } & 1 \mathrm{H} \\ \text { P1 } & 14.75 \text { usec } \\ \text { PL1 } & -1.00 \mathrm{~dB}\end{array}$

PL1W $10.56200695 \mathrm{~W}$

F2 - Processing parameters

$\begin{array}{ll}\text { SI } & 32768 \\ \text { SF } & 400.1300095 \mathrm{MHz}\end{array}$

WDW EM

LB $0.30 \mathrm{~Hz}$

$\begin{array}{lll}\text { GB } & 0 & 0 \\ \text { PC } & 1.00\end{array}$

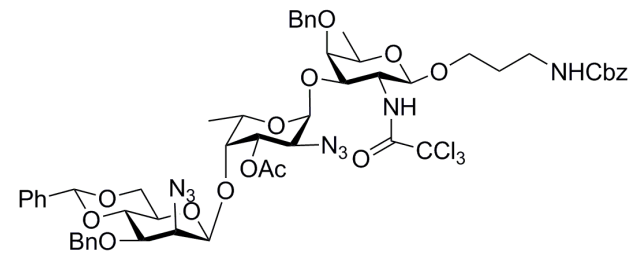

33

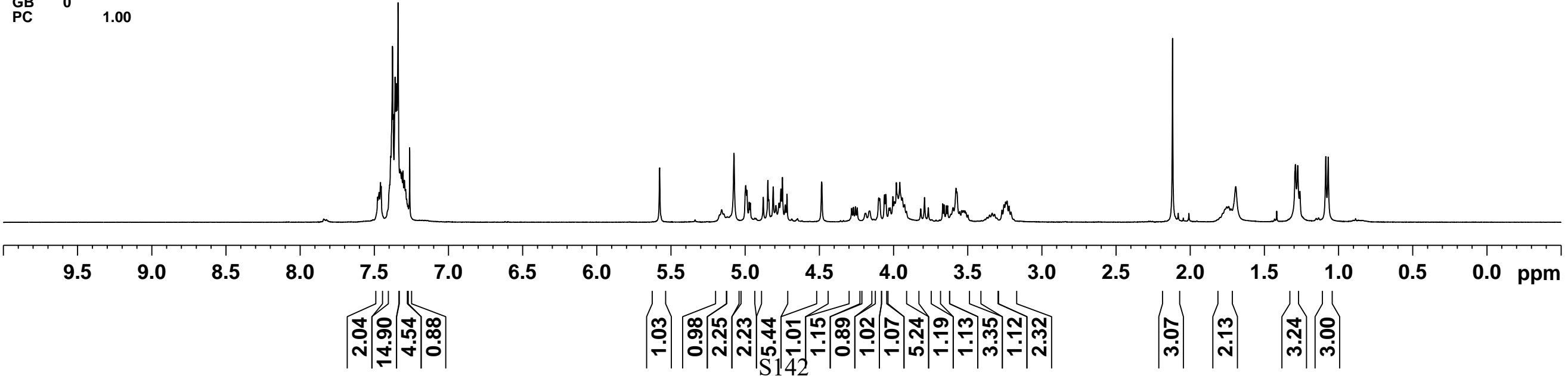




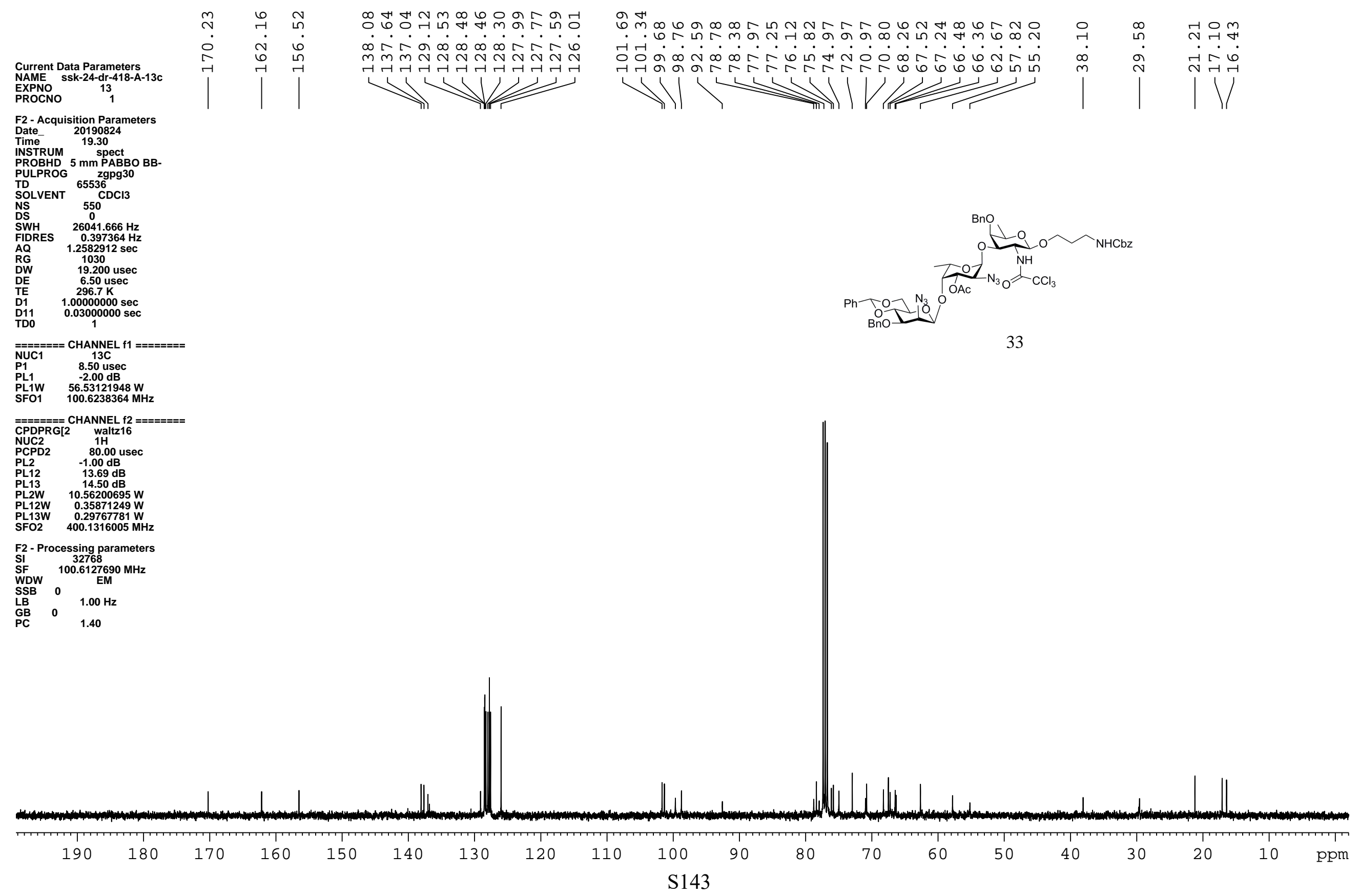


Current Data Parameters
NAME ssk-24-dr-418-A-dept EXPNO 14
1

F2 - Acquisition Parameters
Date_ 20190824

Time-
INSTRUM

PROBHD
PULPROG $\begin{array}{r}\text { mm PABBO BB- } \\ \text { dept135 }\end{array}$

SOLVENT

DS

FWH

$\mathrm{AQ}$
$\mathrm{RG}$
$\mathrm{DW}$

$\mathrm{DW}$
$\mathrm{DE}$
$\mathrm{TE}$

CNST2

$\mathrm{D} 1$

D2

$=======$ CHANNEL

$=======$ CHANNEL $f 1$ = $======$
NUC1 $13 \mathrm{C}$ 8.75 usec
17.50 usec $56.53121948 \mathrm{~W}$
$100.6242389 \mathrm{MHz}$ SF01 $===== \pm$ CHANNEL $\mathrm{f} 2====$
WPDPRG $[2 \quad 1 \mathrm{l} z 16$

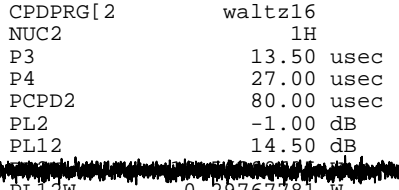

PLI2W M.

F2 - Processing parameters

SI $\quad 100.6127690 \mathrm{MHz}$

$\begin{array}{llr}\text { WDW } & & 100.612769 \\ \text { SSB } & 0 & \text { EM } \\ \text { LB } & & 1.00 \mathrm{~Hz}\end{array}$

$\begin{array}{lll}\mathrm{LB} & 0 & 1.00 \\ \mathrm{GC} & & 1.40\end{array}$
ก

๑

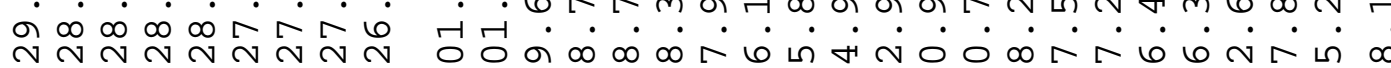

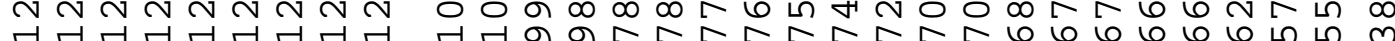

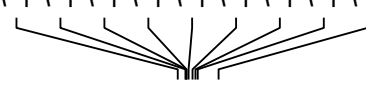

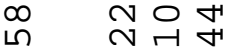

คे $\quad$ iñ
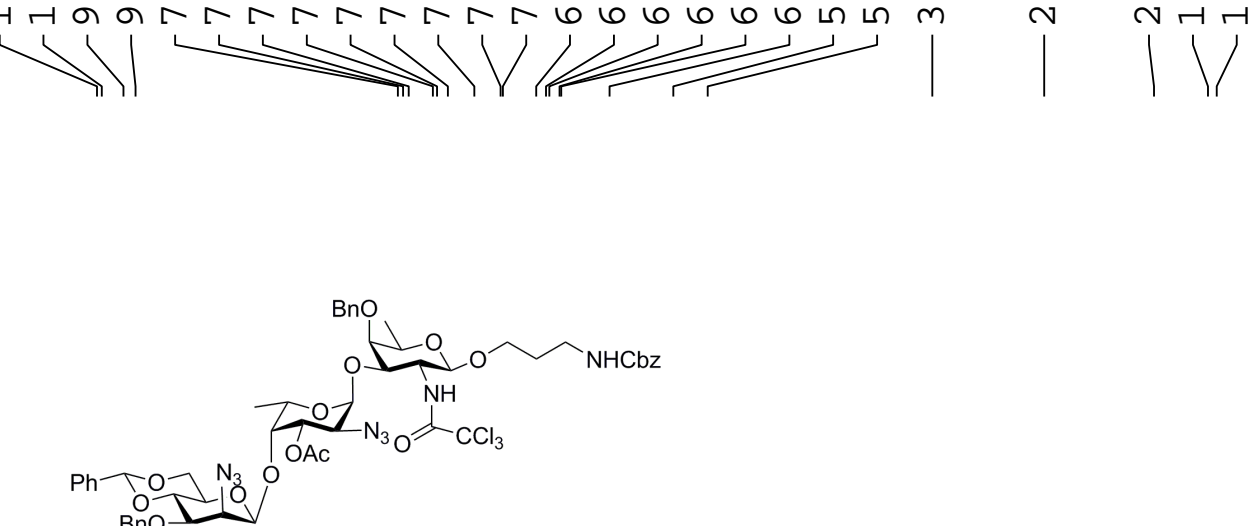

33

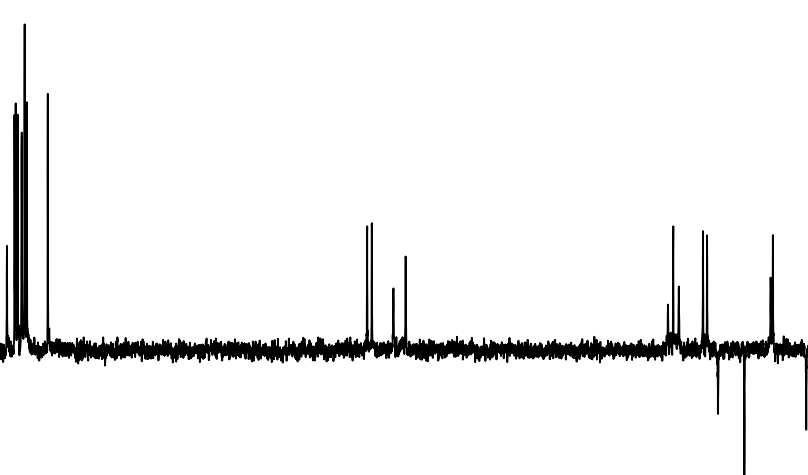

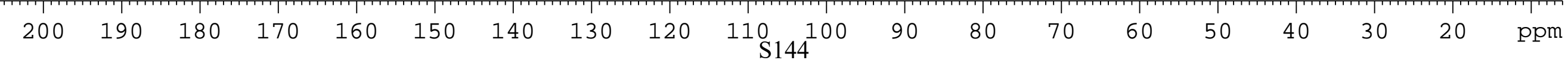




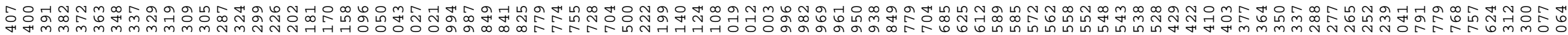

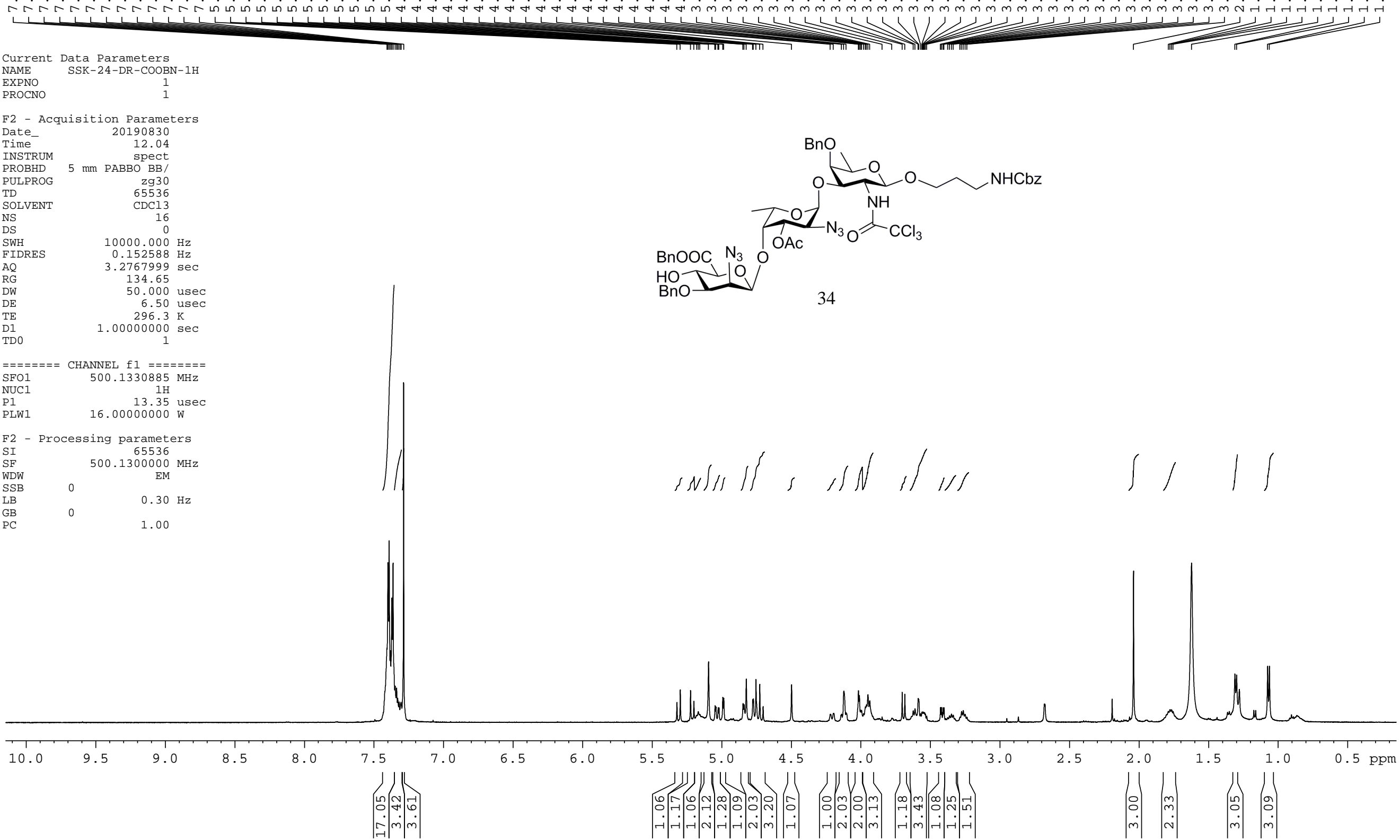




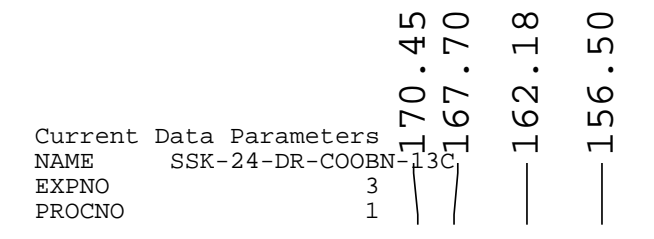

F2 - Acquisition Parameters

Date 20190830

INSTRUM

PROBHD

PULPROG
TD
SOLVENT

SOLVE
NS

DS

SWH

AQ

RW

DE

$\begin{array}{ll}\text { D1 } & 1.00000000 \mathrm{sec} \\ \text { D11 } & 0.03000000 \mathrm{sec}\end{array}$

$=======$ CHANNEL f1 $========$
SFO1 $\quad 125.7703637 \mathrm{MHz}$
P1

$\begin{array}{lr}\text { NUC1 } & 13 \mathrm{C} \\ \text { P1 } & 8.90 \text { usec } \\ \text { PLW1 } & 103.00000000 \mathrm{~W}\end{array}$

$=====$
SFO2
NUC2
CPDPRG

CPDPRG [ 2

PCPD2
PLW2

PLW12
PLW13

F2 - Processing parameters

F2 - Processing parameters
SI
SF

\begin{tabular}{l|l} 
SF & $125.7577890 \mathrm{MH}$ \\
WDW &
\end{tabular}

$\begin{array}{lll}\text { SSB } & 0 & 1.00 \mathrm{~Hz} \\ \text { LB } & 0\end{array}$

$\begin{array}{lll}\text { GB } & 0 & 1.40\end{array}$
๒๐ำ

$\dot{\infty} \dot{\sim} \dot{\sim} \infty \dot{\infty} \infty \dot{\infty} \dot{\infty} \dot{\sim}$

m $m \sim N \cap N \cap N$

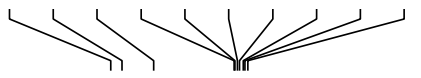

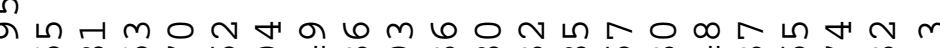
$\dot{0} \infty$ !

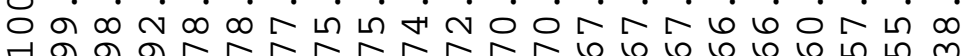
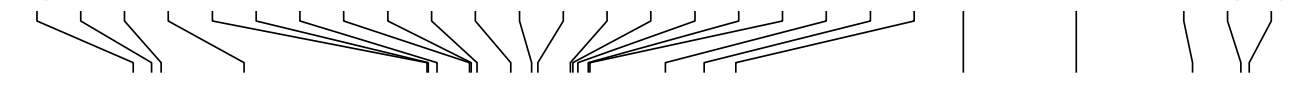
BnOOC $\mathrm{N}_{3} \overbrace{\mathrm{OAC}} \mathrm{OA} \mathrm{N}_{3} \mathrm{O}-{ }_{\mathrm{CCl}_{3}}^{\mathrm{NH}}$

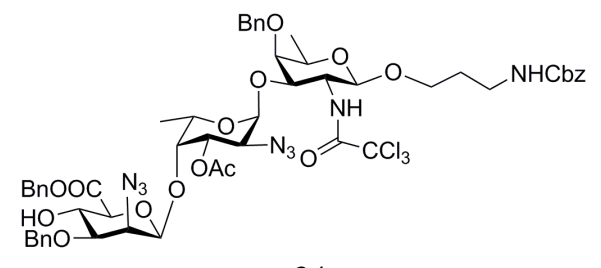

34

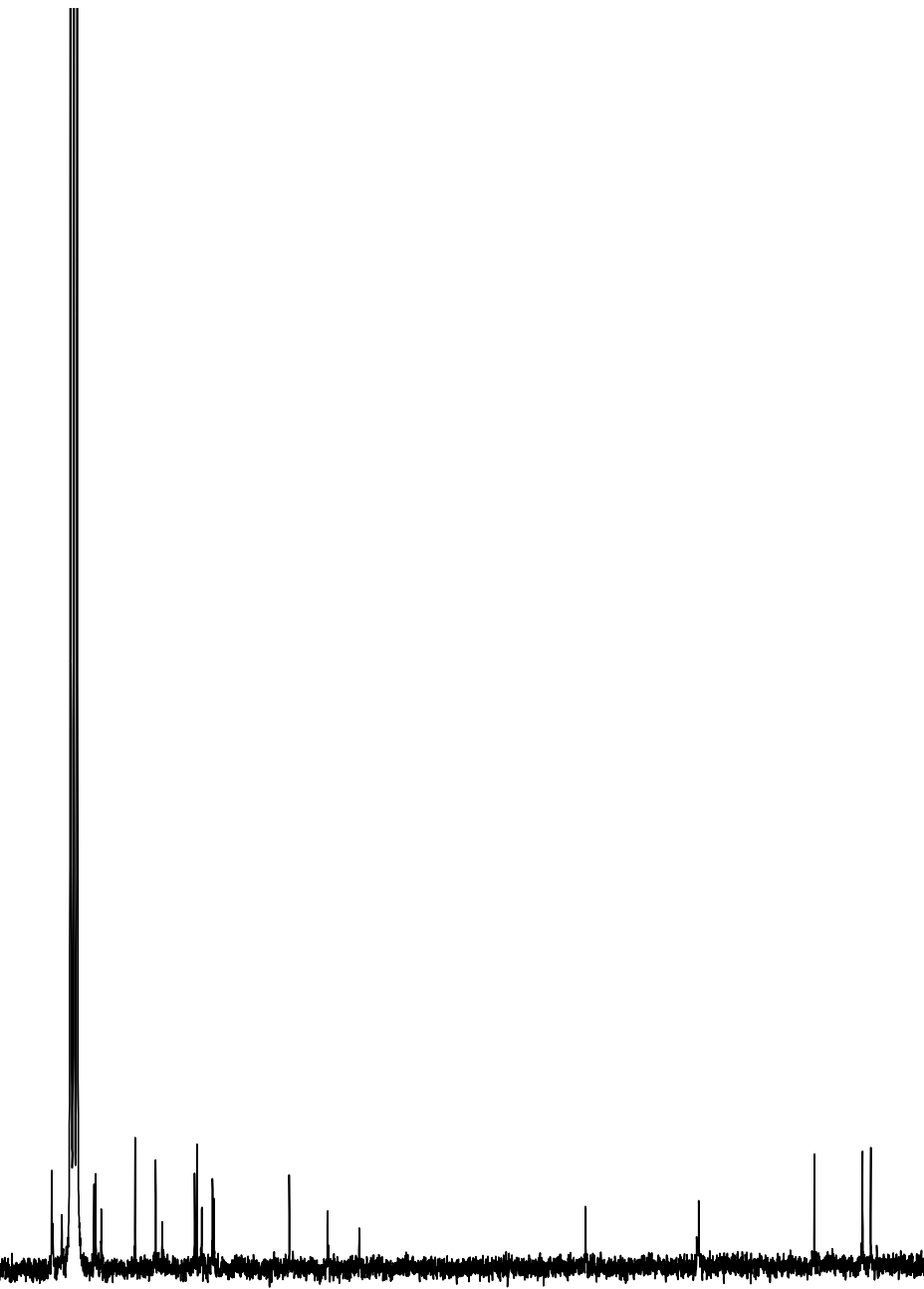

190 
SSK-2 4-DR-COOBN-DEPT

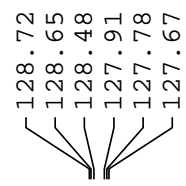

BnOOC ${ }^{2}$

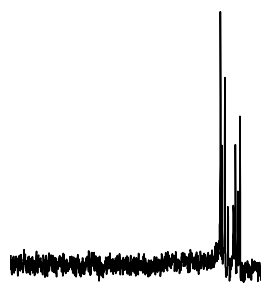


Current Data Parameters

NAME ssk-24-dr-400-1h

EXPNO

F2 - Acquisition Parameters

Date 20190718

Time $\quad 10.01$

INSTRUM $\begin{gathered}\text { spect } \\ \text { PROBHD } 5 \mathrm{~mm} \text { PABBO BB }\end{gathered}$

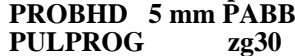

PULPROG

SOLVENT D2O

DS

FIDRES

$\quad 10000.000 \mathrm{~Hz}$

DW $\mathbf{5 0 . 0 0 0}$ usec

D1 $\quad 1.00000000$

TD0 1.000000

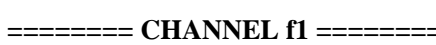

SFO1 500.1330885 MHz

NUC1 $1 \mathrm{H}$

PLW1 $16.00000000 \mathrm{~W}$

F2 - Processing parameters

$\begin{array}{lc}\text { SI } & 65536 \\ \text { SF } & \mathbf{5 0 0 . 1 2 9 9 5 3 1} \mathrm{MHz}\end{array}$

WDW EM

$\begin{array}{lll}\text { LSB } & 0 & 0.30 \mathrm{~Hz}\end{array}$

$\begin{array}{lll}\text { GB } & 0 & \\ \text { PC } & & 1.00\end{array}$
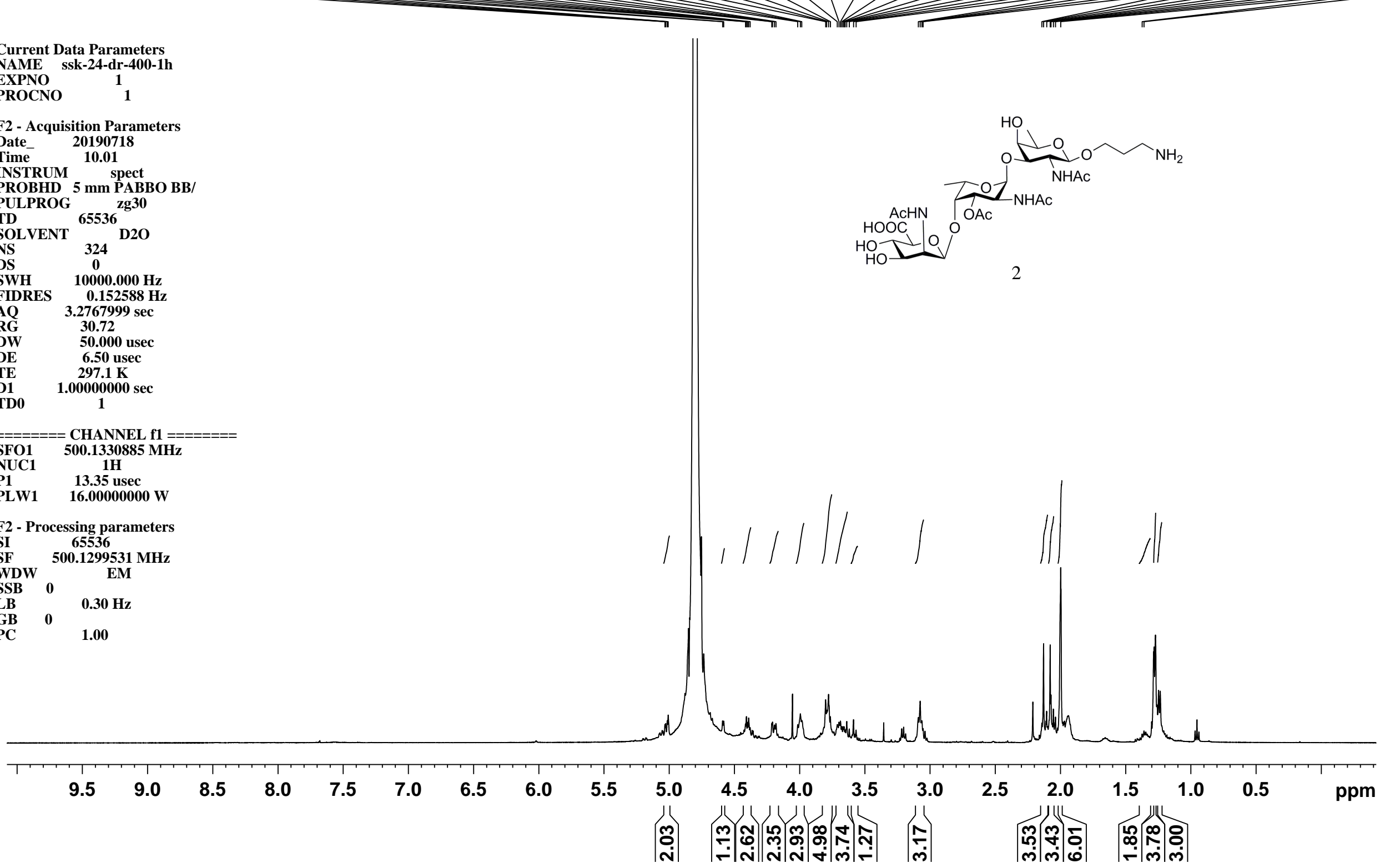


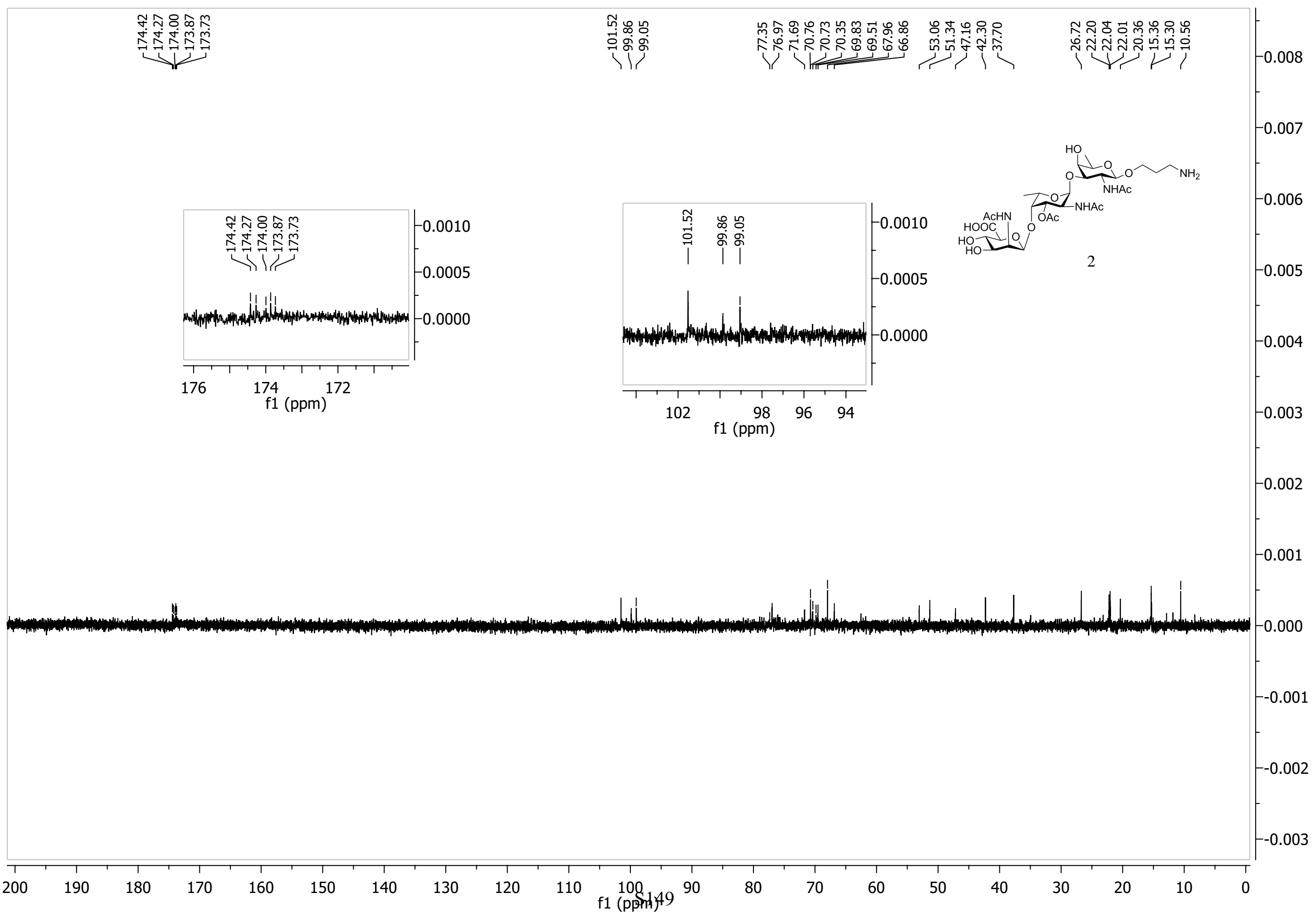




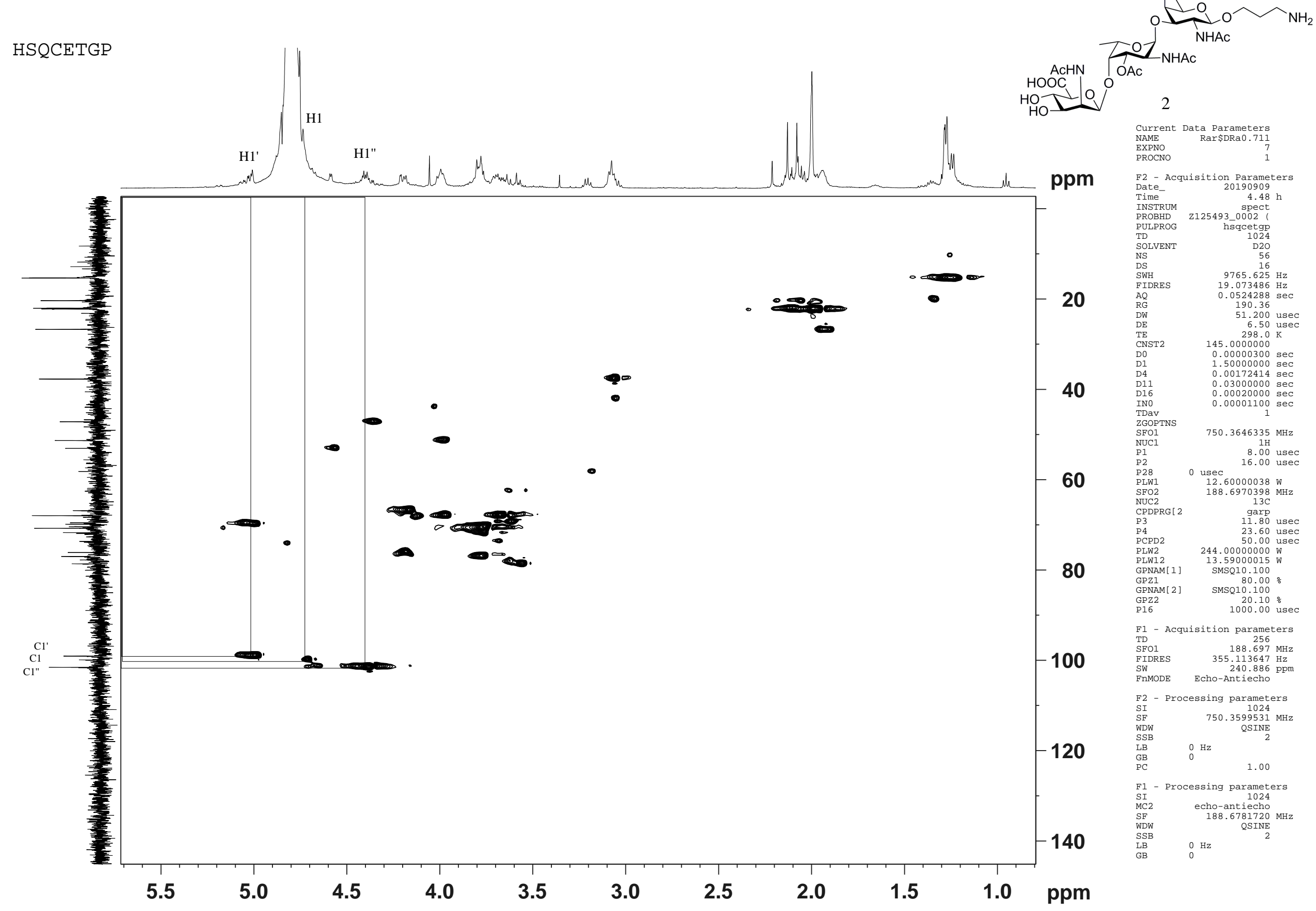

